\title{
METODOLOGIA PARA DEPURAÇÃO OFF-LINE DE PARÂMETROS SÉRIE E SHUNT DE LINHAS DE TRANSMISSÃO ATRAVÉS DE DIVERSAS AMOSTRAS DE MEDIDAS
}

MADELEINE ROCIO MEDRANO CASTILLO ALBERTINI

Tese de Doutorado apresentada à Escola de Engenharia de São Carlos, da Universidade de São Paulo, como parte dos requisitos para a obtenção do Título de Doutora em Ciências, programa de Engenharia Elétrica.

Área de Concentração: Sistemas Elétricos de Potência

ORIENTADOR: Prof. Dr. João Bosco A. London Junior

CO-ORIENTADOR: Prof. Dr. Newton Geraldo Bretas

São Carlos

2010 
AUTORIZO A REPRODUCĀO E DIVULGACĀO TOTAL OU PARCIAL DESTE TRABALHO, POR QUALQUER MEIO CONVENCIONAL OU ELETROONICO, PARA FINS DE ESTUDO E PESQUISA, DESDE QUE CITADA A FONTE.

Ficha catalográfica preparada pela Seção de Tratamento da Informação do Serviço de Biblioteca-EESC/USP

$2.334 \pi$

Albertini, Madeleine Roci० Medrano Castillo

$$
\text { Met } \propto \text { ologia para depuraça off-line de parâmetros }
$$
série e shunt de linhas de transmissāo através de diversas amostras de medidas / Madeleine Rocio Medrano Castillo Albertini; orientador Joāo Bosco A. London Junior, co-orientador Newton Geraldo Bretās. -- Sāo Carlos, 2010 .

Tese (Doutorado-Programa de Pós-Graduacāo en Engenharia Elétrica e prea de Concentraça en Sistemas Elêtricos de Potência) -- Escola de Engenharia de São Carlos da Universidade de Sāo Paulo, 2010.

1. Sistenas elétricos de potência. 2. Estimaça o de estado. 3. Parânetro de linhas de transnissāo. 4. Análise da observabilidade. 5. Erros topológicos. 6. Erros grosseiros. I, Bretas, Newton Geraldo. II. Título. 
FOLHA DE JULGAMENTO

Candidato(a): Ingenieria MADELEINE ROCIO MEDRANO CASTILLO ALBERTINI.

Tese defendida e julgada em 08.09.2010 perante a Comissão Julgadora:

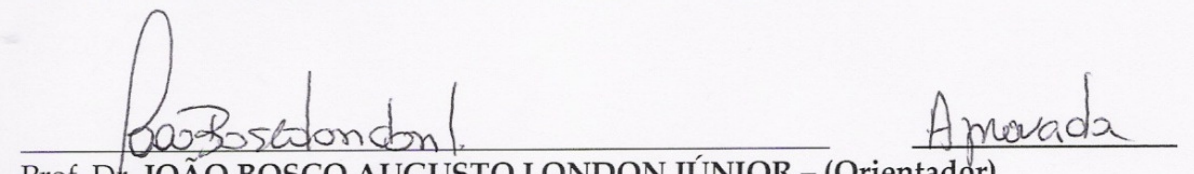

Prof. Dr. JOÃO BOSCO AUGUSTO LONDON JÚNIOR - (Orientador)

(Escola de Engenharia de São Carlos/USP)

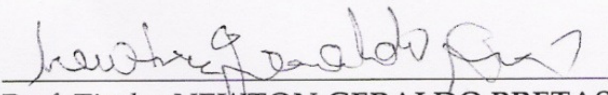

Prof. Titular NEWTON GERALDO BRETAS - (Co-orientador)

(Escola de Engenharia de São Carlos/USP)
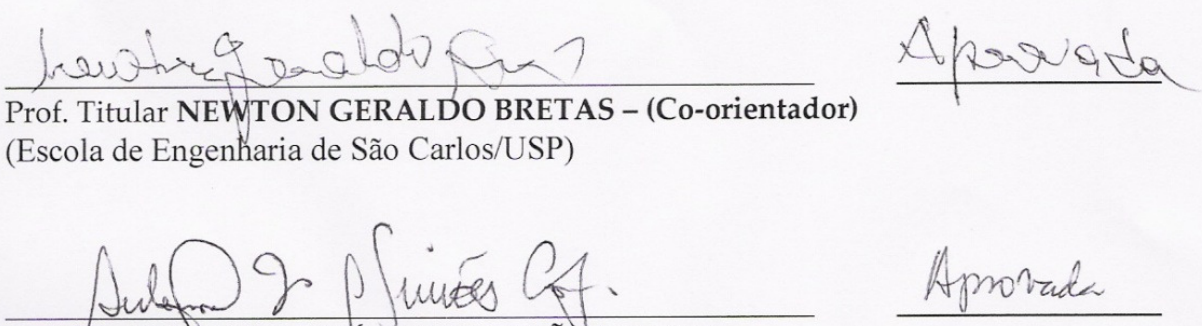

Prof. pr. ANTONIO JOSÉ ALVES SIMÕES COSTA

(Universidade Federal de Santa Catarina/UFSC)
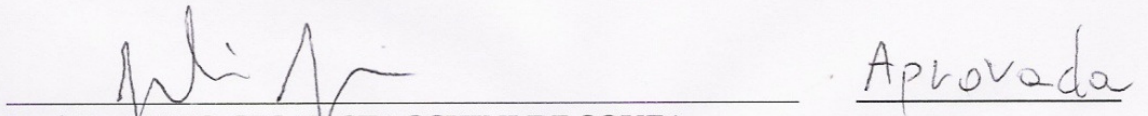

Prof. Dr. JULIO CESAR STACCHINI DE SOUZA

(Universidade Federal Fluminense/UFF)

\section{Burmeth}

$A P R O A D_{0}$

Prof. Dr. ARNUEFO BARROSO VASCONCELLOS

(Universidade Federal de Mato Grosso/UFMT)

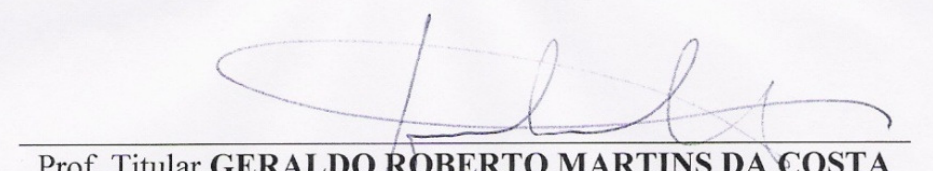

Prof. Titular GERALDO ROBERTO MARTINS DA COSTA

Coordenador do Programa de Pós-Graduação em Engenharia Elétrica e Presidente da Comissão de Pós-Graduação 


\section{Dedicatória}

Com muito carinho dedico este trabalho: aos meus queridos pais, Lucio e Maria Pilar, aos meus irmãos, Flor Rosário, Yenny Esperanza e Lucio Abimael, a meu esposo Marcelo. 


\section{Agradecimentos}

A Deus, por tudo.

Ao meu orientador professor João Bosco Augusto London Junior, pela amizade, confiança

e orientação, tanto científica quanto pessoal, pelo apoio e dedicação oferecidos na elaboração desta tese. Pelos bons conselhos dados durante todos estes anos.

Ao meu co-orientador professor Newton Geraldo Bretas pela amizade, orientação e valiosas sugestões para realização desta tese.

A Hydro-Québec, em especial à equipe do Dr. Serge Lefebvre do Institut de Recherche d'Hydro-Québec, pela amizade, pelas discussões científicas e profissionais, e pelo bom ambiente de trabalho.

Aos meus pais, Lucio Medrano Aguirre e Maria Pilar Castillo de Medrano, por acreditar em mim e estar sempre do meu lado. Muito obrigado por tudo pais.

Ao meu esposo Marcelo Keese Albertini, pelo seu amor, compreensão e apoio ao longo destes anos.

Aos amigos do LACOSEP (Laboratório de Análise Computacional em Sistemas Elétricos de Potência), pela amizade, pelas discussões profissionais, e pelo bom ambiente de trabalho.

À Fundação de Amparo à Pesquisa do Estado de São Paulo (FAPESP) pela concessão da bolsa de doutorado e pelo apoio financeiro para a realização dessa pesquisa. 


\section{Resumo}

Albertini, M. R. M. C. Metodologia para Depuração off-line de parâmetros série e shunt de linhas de transmissão através de diversas amostras de medidas. 2010. Tese (Doutorado)Departamento de Engenharia Elétrica da Escola de Engenharia de São Carlos, Universidade de São Paulo, São Carlos, 2010.

Neste trabalho propõe-se uma metodologia off-line, prática e eficiente, para detectar, identificar e corrigir erros em parâmetros série e shunt de linhas de transmissão. As linhas de transmissão, ou ramos do modelo barra-ramo, suspeitas de estarem com EPs são identificadas através do Índice de Suspeita (IS). O IS de um ramo é a relação entre o número de medidas incidentes a esse ramo, cujos resíduos normalizados são maiores que um valor pré-estabelecido, e o número total de medidas incidentes a esse ramo. Usando várias amostras de medidas, os parâmetros dos ramos suspeitos são estimados, de forma seqüencial, via um estimador de estado e parâmetros baseado nas equações normais, que aumenta o vetor de variáveis de estado para inclusão dos parâmetros suspeitos. Resultados numéricos de diversas simulações, com os sistemas de 14, 30 e 57 barras do IEEE, têm demonstrado a alta precisão e confiabilidade da metodologia proposta, mesmo na ocorrência de erros múltiplos (em mais de um parâmetro) em ramos adjacentes, como também em linhas de transmissão paralelas com compensação série. Comprovou-se a viabilidade prática da metodologia proposta através da aplicação da mesma, para depuração (detecção, identificação e correção) dos valores dos parâmetros de dois subsistemas da Hydro-Québec Trans-Énergie.

Palavras- Chave: Sistemas Elétricos de Potência, Estimação de Estado, Detecção e Identificação de Erros de Parâmetros, Estimação de Parâmetros. 


\section{Abstract}

Albertini, M. R. M. C. Methodology for off-line validation of transmission line parameters via several measurement snapshots. Tese (Doctoral)- Departamento de Engenharia Elétrica da Escola de Engenharia de São Carlos, Universidade de São Paulo, São Carlos, 2010.

A practical and efficient off-line approach to detect, identify and correct series and shunt branch parameter errors is proposed in this thesis. The branches suspected of having parameter errors are identified by means of the Suspicious Index (SI). The SI of a branch is the ratio between the number of measurements incident to that branch, whose normalized residuals are larger than one specified threshold value, and the total number of measurements incident to that branch. Using several measurement snapshots, the suspicious parameters are sequentially estimated, via an augmented state and parameter estimator which increases the $\mathrm{V}-\theta$ state vector for the inclusion of suspicious parameters. Several simulation results (with IEEE 14, 30 and 57 bus systems) have demonstrated the high accuracy and reliability of the proposed approach to deal with single and multiple parameter errors in adjacent and non-adjacent branches, as well as in parallel transmission lines with series compensation. The proposed approach is confirmed by tests performed in two subsystems of the Hydro-Québec Trans-Énergie.

Index Terms-Electric Power Systems, State Estimation, Parameter Error Detection and Identification, Parameter Estimation. 


\section{Lista de Figuras}

Figura 1.1 Configuração do Sistema SCADA/EMS............................................... 2

Figura 2.1 Visão geral de um SPMS................................................................... 27

Figura 2.2 Estrutura básica de uma PMU................................................................ 28

Figura 4.1 Modelo $\Pi$ - Equivalente generalizado................................................. 60

Figura 4.2 Sistema teste de 3 barras................................................................ 78

Figura 4.3 Fluxograma do algoritmo proposto.................................................. 83

Figura 5.1 Sistema de 14 barras do IEEE associado a um conjunto redudante de

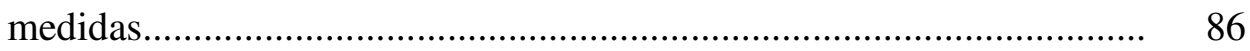

Figura 5.2 Sistema de 57 barras do IEEE associado a um conjunto redudante de medidas...

Figura 5.3 Sistema de 30 barras do IEEE associado a um conjunto redundante de medidas.

Figura 6.1 Subsistema 1 de Hydro-Quebéc.

Figura 6.2 Subsistema 2 de Hydro-Quebéc.

Figura 6.3 Representação de uma linha do subsistema 2...

Figura 6.4 Relação dos índices de desempenho obtidos a partir de amostras de medidas do dia 24 de setembro $J^{1}(X) / J^{2}(X)$-Subsistema 1 .

Figura 6.5 Relação dos índices de desempenho obtidos a partir de amostras de medidas do dia 26 de setembro $J^{1}(X) / J^{2}(X)$-Subsistema 2 . 


\section{Lista de Tabelas}

Tabela 3.1 Discrepâncias entre fontes de dados de parâmetros.............................. 34

Tabela 5.1 Parâmetros de linhas do sistema de 14 barras do IEEE......................... 87

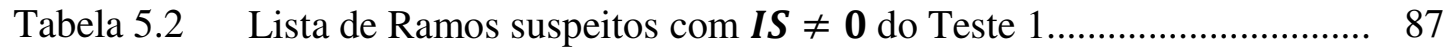

Tabela 5.3 Parâmetros estimados do Teste 1....................................................... 88

Tabela 5.4 Comparação de estimativas - Teste 1................................................. 89

Tabela 5.5 Lista 1 de Ramos suspeitos $\operatorname{com} \boldsymbol{I} \boldsymbol{S} \neq \mathbf{0}$ Teste-2 2.............................. 90

Tabela 5.6 Lista 2 de Ramos suspeitos $\operatorname{com} \boldsymbol{I} \boldsymbol{S} \neq \mathbf{0}$ Teste-2............................ 90

Tabela 5.7 Parâmetros estimados do Teste 2...................................................... 92

Tabela 5.8 Comparação de estimativas - Teste 2 2.............................................. 92

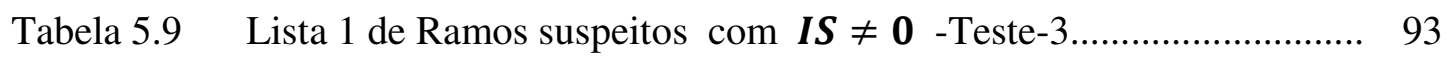

Tabela 5.10 Lista 2 de Ramos suspeitos $\operatorname{com} \boldsymbol{I} \boldsymbol{S} \neq \mathbf{0}-$ Teste-3.......................... 94

Tabela 5.11 Lista 3 de Ramos suspeitos com $\boldsymbol{I} \boldsymbol{S} \neq \mathbf{0}$ - Teste-3.......................... 95

Tabela 5.12 Parâmetros estimados do teste 3 ...................................................... 96

Tabela 5.13 Comparação de estimativas - Teste 3 ............................................... 96

Tabela 5.14 Lista 1 de Ramos suspeitos com $\boldsymbol{I} \boldsymbol{S} \neq \mathbf{0}$ - Teste-4 …..................... 98

Tabela 5.15 Parâmetros Estimados do Teste 4 ....................................................... 98

Tabela 5.16 Comparação de estimativas - Teste 4 _................................................ 98

Tabela 5.17 Lista 1 de Ramos suspeitos com $\boldsymbol{I} \boldsymbol{S} \neq \mathbf{0}-$ Teste-5 ......................... 100

Tabela 5.18 Lista 2 de Ramos suspeitos com $\boldsymbol{I} \boldsymbol{S} \neq \mathbf{0}$ - Teste-5 .......................... 101

Tabela 5.19 Parâmetros estimados do Teste 5 ..................................................... 101

Tabela 5.20 Comparação de estimativas - Teste 5 .............................................. 101

Tabela 5.21 Lista 1 de Ramos suspeitos com $\boldsymbol{I} \boldsymbol{S} \neq \mathbf{0}$ - Teste-6 ......................... 103

Tabela 5.22 Parâmetros estimados do Teste 6 ....................................................... 103

Tabela 5.23 Comparação de estimativas - Teste 6.................................................. 103

Tabela 5.24 Lista 1 de Ramos suspeitos com $\boldsymbol{I S} \neq \mathbf{0}$ - Teste-7 .......................... 105

Tabela 5.25 Parâmetros estimados do Teste 7 ........................................................ 105

Tabela 5.26 Comparação de estimativas - Teste 7 .................................................. 106 
Tabela 5.27 Parâmetros de linhas do sistema de 30 barras do IEEE ....................... 108

Tabela 5.28 Resultados referentes à situação 1 (metodologia proposta)................... 110

Tabela 5.29 Resultados referentes à situação 2 (metodologia proposta).................. 110

Tabela 5.30 Resultados referentes à situação 1 (estimador proposto em LIU;WU 110 (1992)

Tabela 5.31 Resultados referentes à situação 2 (estimador proposto em LIU; WU 111 (1992)

Tabela 5.32 Parâmetros estimados do Teste 9....................................................... 111

Tabela 6.1 Primeira lista de ramos suspeitos - Subsistema 1............................ 117

Tabela 6.2 Resultados Parciais -Subsistema 1................................................. 118

Tabela 6.3 Segunda lista de ramos suspeitos - Subsistema................................. 118

Tabela 6.4 Resultados Finais -Subsistema 1................................................... 118

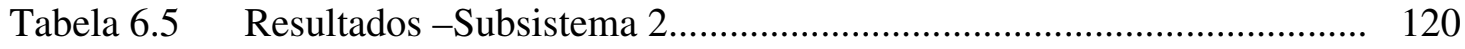

Tabela B.1 FE de medidas de potência do sistema de 14 barras do IEE................. 148

Tabela B.2 Parâmetros dos medidores................................................................. 151

Tabela B.3 Fundo de escala para medidores de potencia do sistema de 14 barras.. 152

Tabela B.4 Fundo de escala para medidores de potencia do sistema de 30 barras.. 153

Tabela B.5 Fundo de escala para medidores de potencia do sistema de 57 barras.. 154 


\section{Lista de Abreviaturas e Siglas}

SEP Sistemas Elétricos de Potência

SPMS Synchronized Phasor Measurement Systems

WAMS Wide Area Measurement System

PMU Phasor Measurement Units

PDC Phasor Data Concentrator

COS Centros de Operação do Sistema

SCADA Supervisory Control and Data Aquisition

EMS Energy Management System

IED Inteligent Electronic Devices

UTR Remote Terminal Unite

EESEP Estimação de Estado em Sistemas Elétricos de Potência

EP Estimação de Parâmetros

EG Erro Grosseiro

IS Índice de Suspeita

LRS Lista de Ramos Suspeitos

TM Número Total de Medidas disponíveis incidentes ao ramo i

WLS Weight Least Square

IEEE Institute of Electrical and Electronic Engineer

FASE Forecasting-Aided State Estimators

GPS Global Position System 


\section{Lista de Símbolos}

$G_{k m} \quad$ Condutâncias série de Linha de Transmissão Aéreas

$B_{k m} \quad$ Susceptâncias série de Linhas de Transmissão Aéreas

Z Vetor de medidas

$h($.) Vetor de funções não lineares

$x_{v} \quad$ Vetor das variáveis de estado verdadeiras

w Vetor dos erros das medidas

$m \quad$ Numero de medidas

$n \quad$ Numero de variáveis de estado a serem estimadas

W Matriz de ponderação para as medidas

$J(x) \quad$ Função Quadrática

$H(x) \quad$ Matriz Jacobiana

$\Delta z \quad$ Erro de estimação

$r \quad$ Vetor resíduo de estimação

$\Gamma \quad$ Matriz sensibilidade do resíduo

I Matriz Identidade

$R \quad$ Matriz de covariância

$\tau_{i} \quad$ Resíduos normalizados

$\rho_{i i} \quad$ Desvio Padrão unitário

$b_{j} \quad$ Erro grosseiro da medida $\mathrm{j}$

- $\quad$ Média do resíduo de estimação

$\gamma_{i j} \quad$ Elemento $(\mathrm{i}, \mathrm{j})$ da matriz $\Gamma$

$\tau_{i}^{\max } \quad$ Maior resíduo normalizado

$\alpha \quad$ Limite de identificação

$P_{k m} \quad$ Fluxo de potencia ativa, da barra k para a barra m

$Q_{k m} \quad$ Fluxo de potencia reativa, da barra k para a barra m 
$G_{\theta} \quad$ Matriz Ganho

$H_{P \theta} \quad$ Matriz Jacobiana, relacionada apenas às medidas de potência ativa

$Z_{p} \quad$ Vetor de medidas de potência ativa

$W_{p} \quad$ Matriz de ponderação das medidas de potência ativa

$V_{k} \quad$ Magnitude de tensão da barra k

$V_{m} \quad$ Magnitude de tensão da barra m

$\theta_{k m} \quad$ Angulo de defasagem entre a barra k e a barra m

$n b \quad$ Numero de barras

$U_{\theta} \quad$ Matriz ganho superior fatorada

$Z_{\text {Aum }} \quad$ Vetor de medidas aumentado

$h_{\text {Aum }}($.) Vetor de funções não lineares, que relaciona as medidas com as variáveis de estado aumentado.

$X_{v A u m} \quad$ Vetor de estado aumentado verdadeiro

$w_{\text {Aum }} \quad$ Vetor dos erros nas medidas

$n_{\text {Aum }} \quad$ Número de variáveis de estado e parâmetros, a ser estimado.

$m_{\text {Aum }} \quad$ Número de medidas selecionadas na Fase1.

L Ramos

$H_{\text {Aum }} \quad$ Matriz Jacobiana aumentada

$W_{\text {Aum }} \quad$ Matriz de Ponderação das medidas aumentadas

$y_{k m} \quad$ Admitancia série da linha de transmissão k-m

$y_{k m}^{s h} \quad$ Susceptância shunt

$a_{k m} e^{j \theta p} \quad$ A relação de transformação

$G_{\text {Aum }} \quad$ Matriz ganho aumentada 


\section{Conteúdo}

Dedicatória $\quad$ iii

Agradecimentos $\quad$ v

Resumo vii

Abstract $\quad$ ix

Lista de Figuras $\quad$ xi

Lista de Tabelas $\quad$ xii

Lista de Abreviaturas e Siglas $\quad$ xiv

Lista de Símbolos $\quad$ xv

1 Introdução 1

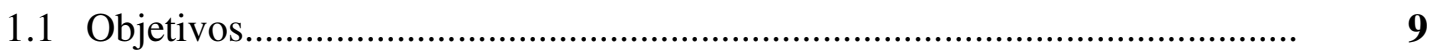

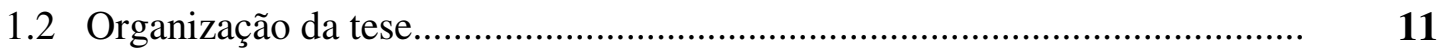

\section{Estimação de Estado em Sistemas Elétricos de Potencia}

2.1 Introdução........................................................................................... 13

2.2 Etapas do processo de Estimador de Estado em Sistemas Elétricos de

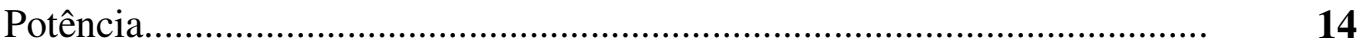

2.2.1 Etapa 1: Obtenção da topologia do sistema, no modelo barra-ramo... $\quad \mathbf{1 5}$

2.2.2 Etapa 2: Análise e restauração da observabilidade do sistema............ $\quad 15$

2.2.3 Etapa 3: Estimação de Estado............................................................... 16

2.2.4 Etapa 4: Processamento de Erros Grosseiros em Medidas

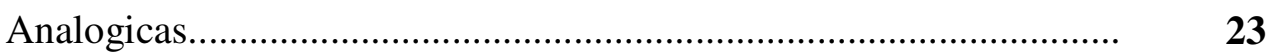

2.3 Sistema de Medição Fasorial Sincronizada.................................................. 27

2.3.1 Unidades de Medição Fasorial -PMUs................................................ 28

2.3.2 Aplicação em Estimação de estado.................................................... 30 
3 Erros de Parâmetros no Processo de Estimação de Estado em Sistemas Elétricos de Potência

3.1 Influencia de erros de parâmetros no processo de estimação de estado em

Sistemas Elétricos de Potência.

3.2 Detecção e identificação de erros de parâmetros

3.3 Classificação dos métodos para o tratamento de erros de parâmetros........... 39

3.3.1 Métodos que utilizam Equações Normais.......................................... 45

3.3.2 Métodos que utilizam Filtro de Kalman.......................................... $\quad \mathbf{5 4}$

3.3.3 Soluções alternativas para estimação de parâmetros......................... $\quad \mathbf{5 8}$

3.4 Comparação entre as metodologias de tratamento de erros de parâmetros.. $\quad$ 60

4 Metodologia Proposta para Depuração Off-Line de Parâmetros Série e Shunt de Linhas de Transmissão

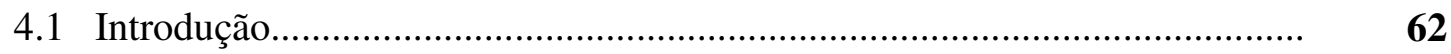

4.2 Parâmetros a serem estimados.......................................................................

4.3 Metodologia Proposta................................................................................

4.3.1 Fase 1: Detecção e Identificação de ramos suspeitos de estarem com erros

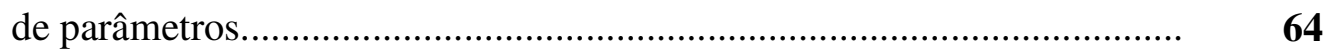

4.3.2 Fase 2: Estimação dos Parâmetros Suspeitos.............................................. $\quad \mathbf{6 5}$

4.3.2.1 Formulação do Estimador Desacoplado de Estado e

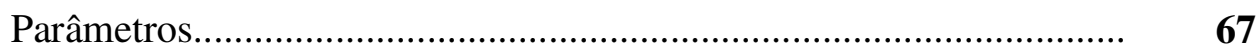

4.3.3 Fase 3: Validação das estimativas dos parâmetros suspeitos..................... $\quad \mathbf{7 9}$

4.3.4 Algoritmo geral para depuração de parâmetros série e shunt de linhas de transmissão.

\section{Testes Realizados e Análise dos resultados}

5.1 Características de implementação.................................................................. 84

5.2 Simulações com o sistema de 14 barras do IEEE............................................ 8

5.3 Simulações com o sistema de 57 barras do IEEE........................................... 99

5.4 Simulações com o sistema de 30 barras do IEEE.......................................... 107

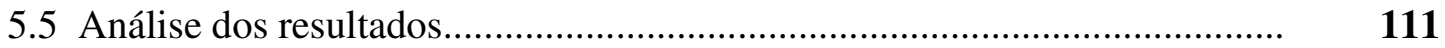


6 Testes realizados com o sistema da Hydro-Quebec Trans-ènergie

6.1 Subsistemas analisados.

6.2 Amostras utilizadas

6.3 Validação dos Resultados Obtidos.

6.4 Teste - Subsistema 1

6.5 Teste - Subsistema 2

6.6 Analise dos Rsultados

\section{Conclusões e Trabalhos Futuros de Pesquisa}

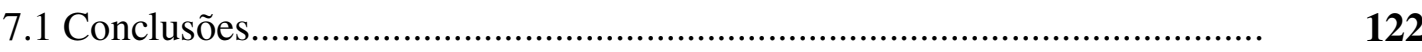

7.2 Considerações finais e Trabalhos futuros ..................................................... 124

$\begin{array}{lr}\text { Publicações Originadas desta pesquisa } & 120\end{array}$

$\begin{array}{ll}\text { Referências } & 126\end{array}$

Apêndice A - Publicações originadas desta pesquisa................................................. 139

Apêndice B - Obtenção dos dados para simulação.................................................... 143

Apêndice C - Métodos para solução de sistemas de equações não lineares................ 154

Apêndice D- Obtenção dos dados para simulação...................................................... $\mathbf{1 6 0}$

Apêndice E - Escalonamento de linhas e colunas...................................................... $\quad 167$ 


\section{Capítulo 1}

\section{Introdução}

Os processos de desregulamentação ocorridos na indústria de energia elétrica nos últimos anos em diversos países, somados ao impacto do crescimento da demanda de energia elétrica e à contínua incorporação de novas tecnologias de equipamentos, têm aumentado a complexidade operacional dos Sistemas Elétricos de Potência (SEP). Neste cenário, as redes elétricas têm sido operadas em condições cada vez mais próximas de seus respectivos limites de capacidade, potencializando a ocorrência de colapsos envolvendo grandes áreas geográficas quando da ocorrência de perturbações. Um exemplo disso é o blecaute acontecido recentemente entre a noite do dia 10 e a madrugada de 11 de novembro, com impacto em 12 estados brasileiros, além de parte do Paraguai.

Estudos recentes indicam claramente que problemas de monitoração em tempo real, operação, controle, sistemas de comunicação e atrasos na restauração do sistema estão presentes quando da ocorrência de falhas de grande porte no sistema. Conclusões como esta sinalizam a necessidade de um aprimoramento dos instrumentos de monitoração e controle em tempo real para uma operação econômica e confiável do sistema.

Dentre os novos instrumentos que têm sido propostos para enfrentar este desafio, destacam-se aqueles relacionados à tecnologia de Sistemas de Medição Fasorial Sincronizada (SPMS, do inglês Synchronized Phasor Measurement Systems). Os SPMS, geralmente citados na literatura técnica como Wide Area Measurement System (WAMS), são constituídos essencialmente por unidades de medição fasorial (PMUs, do inglês Phasor Measurement Units) conectadas a um concentrador de dados (PDC, do inglês Phasor Data Concentrator) e por metodologias de aplicação envolvendo aspectos de monitoração e controle em tempo real. 
A grande vantagem desta tecnologia esta no dispositivo chamado de PMU, que realiza medições com altas taxas de amostragem (tipicamente 30 fasores por segundo comparado com uma a cada 4 segundos utilizando tecnologia convencional). Para cada medida atribui-se uma etiqueta de tempo tendo como base uma referência temporal comum. Essa etiqueta de tempo permite que os fasores enviados por diferentes empresas sejam alinhados (ou sincronizados) e combinados de maneira a apresentar uma visão do comportamento do sistema elétrico.

A necessidade de uma avaliação da segurança dos sistemas acarreta mudanças nos Centros de Operação do Sistema (COS), exigindo-se destes um incremento constante de eficiência. À medida que aumenta a complexidade de operação da rede elétrica, cresce o número de informações oriundas do Sistema de Aquisição e Supervisão de Dados (SCADA, do inglês Supervisory Control and Data Acquisition) transmitidas ao COS, responsável pela monitoração em tempo real do sistema. Isso vem evidenciando cada vez mais a necessidade do tratamento daquelas informações através de aplicativos computacionais avançados de análise em tempo-real. Segue-se assim a tendência mundial de evolução em direção aos denominados Sistemas de Gerenciamento de Energia (EMS, do inglês Energy Management System), para auxiliar o operador no tratamento das informações e viabilizar a verificação rápida das condições de operação do sistema (ver Figura 1.1).

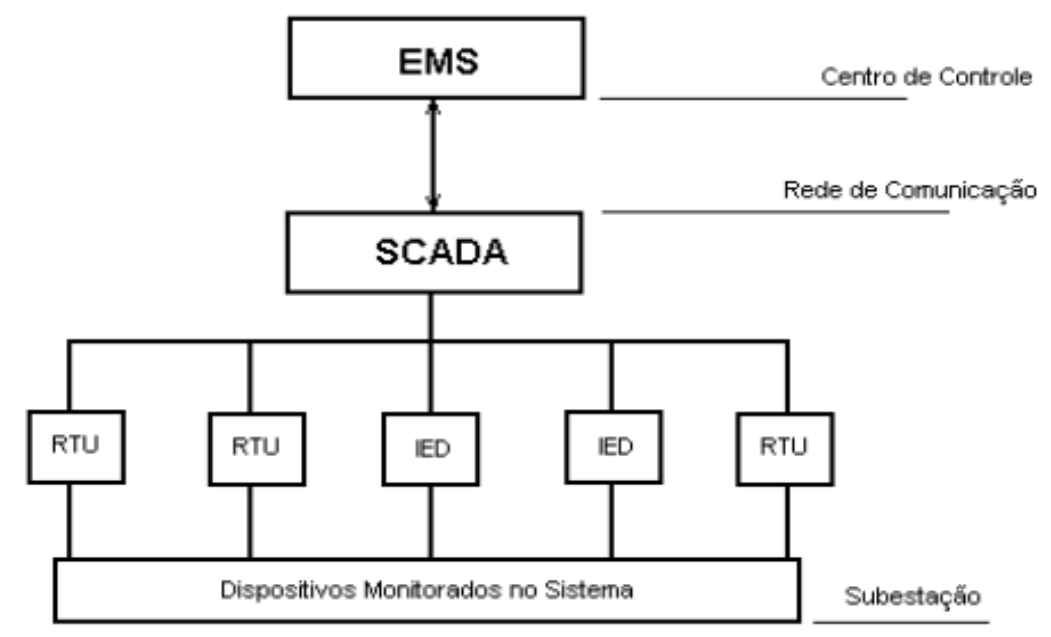

Figura 1.1 - Configuração do sistema SCADA/EMS: RTU 
A aquisição dos dados do SEP se inicia em Unidades Terminais Remotas (RTUs do inglês, Remote Terminal Units), no caso de subestações convencionais modernizadas ou RTUs de poste, ou por Sistemas Digitais de Controle Distribuídos (SDCDs), em subestações completamente digitalizadas. Estes, por sua vez, se comunicam com outros equipamentos inteligentes dispostos na subestação ou usina de energia, como os Controladores Lógicos Programáveis (PLCs) ou Dispositivos Eletrônicos Inteligentes (IEDs do inglês, Intelligent Electronic Devices). Os dados são então transmitidos através de canais de comunicação para o sistema SCADA, com o uso de protocolos de tempo real. No sistema SCADA os dados são compilados e formatados de tal forma que os operadores, utilizando a Interface Homem Máquina (IHM), possam tomar decisões com as informações provenientes das instalações elétricas. O SCADA é composto de diversos subsistemas, quais sejam, o conjunto de equipamentos computacionais e seus programas associados, a infra-estrutura de transmissão de dados e a base de dados de tempo real.

Além da monitoração das condições de operação em tempo-real, os aplicativos componentes do EMS possibilitam diversas análises, dentre as quais podemos destacar: análise de contingências que possam vir a ocorrer no SEP; controle automático de geração; despacho econômico; previsão de carga; e análise de estabilidade dos SEP. Com base nestas análises, os engenheiros tomam decisões relativas às ações de controle cabíveis, tais como: imposição de limites de operação; ajuste dos esquemas de proteção; e alívio de carga. Para que tais decisões possam ser tomadas, de forma a garantir uma operação segura e eficiente do SEP, é necessário que simulações descrevam satisfatoriamente o comportamento real do sistema.

O primeiro e fundamental estágio desta cadeia de iniciativas é a monitoração de segurança, a qual requer uma modelagem precisa da rede elétrica em tempo-real, tendo o estimador de estado como a sua ferramenta computacional mais importante (MONTICELLI, 1999; ABUR; EXPOSITO, 2004). Ao longo dos anos, a função de Estimação de Estado em Sistemas Elétricos de Potência (EESEP), em um Sistema de Gerenciamento de Energia, tornou-se indispensável à construção de uma base de dados completa, coerente e confiável, visando a análise de contingências, cálculo de preço spot, e função de previsão de carga, entre outros. Através desta função, é possível realizar uma 
análise de consistência e depuração de dados, referentes à configuração atual da rede e a grandezas elétricas de interesse para a supervisão.

A finalidade da EESEP é fornecer, em tempo-real, as tensões complexas que representam o ponto atual de operação do sistema, a partir do processamento de informações redundantes obtidas em tempo-real (medidas analógicas ${ }^{1}$, lógicas ${ }^{2}$ ), provenientes do sistema SCADA e/ou dos SPMS, e dos parâmetros do sistema ${ }^{3}$ disponíveis no banco de dados das companhias de energia elétrica.

O problema de EESEP é comumente formulado baseando-se em várias suposições, as quais são:

1) medidas com erros aleatórios gaussianos e não correlacionados, com média zero e matriz de covariância diagonal conhecida;

2) sistema trifásico balanceado;

3) nenhum time-skew entre os valores medidos;

4) nenhuma incerteza em relação aos valores dos parâmetros do modelo $\pi$ equivalente das linhas e transformadores;

5) uma topologia conhecida da rede;

Entretanto, freqüentemente tais suposições não são verdadeiras.

Suspeita-se da existência de erro, no valor de um determinado parâmetro, quando as medidas incidentes a ele apresentam resíduos normalizados elevados.

Parâmetros de elementos da rede, armazenados na base de dados, podem estar incorretos em função de: dados imprecisos fornecidos por fabricantes; estimativa grosseira do comprimento de linhas de transmissão; alterações de projeto, não atualizadas na base de dados (por exemplo, seção de linha aérea que se torna subterrânea); preenchimento incorreto da base de dados; entre outros. A presença de erros em parâmetros degrada os resultados da função EESEP, fazendo com que telemedidas corretas, porém incidentes a tais parâmetros, sejam interpretadas como suspeitas de portar erros grosseiros ${ }^{4}$ (EGs).

\footnotetext{
${ }^{1}$ Usualmente são medidas de fluxo de potência ativa e reativa nas linhas, injeção de potência ativa e reativa e algumas medidas de magnitudes de tensão nas barras.

${ }^{2}$ Consistem em estados de chaves e disjuntores.

${ }^{3}$ Impedância de linhas de transmissão, posição de taps de transformadores, entre outros.

${ }^{4}$ Medidas portadoras de erros grosseiros são aquelas com grau de imprecisão muito maior do que é suposto pelo modelo de medição.
} 
A identificação e a correção de erros de parâmetros (EPs) é um problema importante no controle e na operação de SEP. Estes erros conduzem a estimativas de estado imprecisas, e, além disso, não são facilmente identificados pelos métodos convencionais de identificação de EGs. Vale destacar que o uso de parâmetros inadequados pode fazer com que o sistema opere fora da margem de segurança. Por outro lado, o uso de parâmetros mais confiáveis pode aumentar a margem de transferência de potência, trazendo benefícios econômicos ao sistema (KUSIG; GARRISON, 2004).

É drástico o efeito de um erro de parâmetro, para a função EESEP, sendo normalmente intolerável. Entretanto, é menos evidente que os EGs e topológicos (ZARCO; EXPÓSITO, 2000).

Dentre os vários métodos desenvolvidos para o tratamento de EPs, destacam-se aqueles que aumentam o vetor de variáveis de estado (tensões complexas nas barras do sistema) para incluírem os parâmetros suspeitos de estarem com erro, como se tais parâmetros fossem variáveis de estado independentes (ZARCO; EXPÓSITO, 2000; ABUR; EXPOSITO, 2004). Assim, os parâmetros são estimados juntamente com os ângulos e as magnitudes de tensão. Considerando o tratamento que se dá a este modelo aumentado, tais métodos podem ser divididos em dois grupos:

(1) Métodos que utilizam as Equações Normais (ALLAN; LAUGHTON, 1974; LIU; LIM, 1995; LONDON et al., 2004a)

(2) Métodos que utilizam a teoria do Filtro de Kalman (DEBS, 1974; SLUTSKER; CLEMENTS, 1996).

A limitação dos métodos que utilizam as equações normais está relacionada à observabilidade, isto é, raramente o número de medidas disponível é suficiente para estimar o vetor de variáveis de estado aumentado, uma vez que o vetor de variáveis de estado aumenta, para incluírem os parâmetros suspeitos de estarem com erro, mas o conjunto de medidas continua o mesmo.

Na tentativa de superar essa limitação, os métodos que utilizam o Filtro de Kalman aumentam também o vetor de medidas, através de pseudomedidas que correspondem ao vetor de variáveis de estado aumentado estimado no instante anterior. Os métodos que utilizam o Filtro de Kalman processam diversas amostras de medidas de forma seqüencial, para melhorar de forma recursiva as estimativas dos parâmetros. Porém, a necessidade de 
atualização da matriz de covariância dos EPs aumenta muito o custo computacional dos mesmos, principalmente quando é grande a quantidade de parâmetros suspeitos.

Vale ainda destacar o estimador generalizado desenvolvido por (ALSAÇ et al., 1998). Neste estimador o vetor de variáveis de estado convencional ganha novas variáveis de estado, que são os fluxos de potência ativa e reativa, através dos disjuntores e dos ramos cujos parâmetros serão estimados. Novas pseudo-medidas são também introduzidas no modelo, permitindo assim uma melhor discriminação entre EGs, topológicos e EPs. Devido à grande quantidade de informações, envolvidas no processo de estimação generalizada de estado, o estimador generalizado pode tornar-se inviável para aplicação em tempo-real. Em razão disto, os autores indicam a sua utilização apenas em situações especiais, isto é, quando existe a suspeita da existência de erros topológicos ou de parâmetros (ALSAÇ et al., 1998).

No trabalho de (DE ALMEIDA, 2007), a estimação de estado generalizada foi estendida para redes trifásicas de energia elétrica nas quais o desequilíbrio da carga e da rede são considerados.

Destacam-se, a seguir, alguns aspectos relevantes relacionados à questão de EPs na função EESEP (ZARCO; EXPÓSITO, 2000; ABUR; EXPOSITO, 2004):

1. Para obter-se uma estimação de parâmetros confiável, independentemente do método utilizado, requer-se um adequado nível de redundância das medidas na vizinhança dos elementos suspeitos de conterem EPs;

2. Os métodos baseados na análise da sensibilidade dos resíduos das medidas são os mais plausíveis de serem incorporados aos estimadores de estado convencionais. Por outro lado, dentre os métodos que aumentam o vetor de variáveis de estado, aqueles que utilizam as Equações Normais são os mais propícios a se estenderem aos estimadores convencionais, em comparação com os que utilizam a teoria do Filtro de Kalman, porquanto estes requerem rotinas muito diferentes daquelas utilizadas pelos estimadores convencionais; 
3. A estimação da posição de Taps de transformadores, assim como a determinação da posição de chaves e/ou disjuntores, é uma tarefa a realizar-se on-line. Já a estimação dos parâmetros de um ramo, como a indutância e a capacitância de uma linha de transmissão, que permanecem constantes por um longo período de tempo, são tarefas que podem ser realizadas off-line;

4. Os métodos baseados na análise da sensibilidade dos resíduos são executados ao final do processo de estimação de estado e possuem as seguintes vantagens: não degradam o nível de redundância e não exigem rotinas adicionais às já existentes para implementação do processo convencional de estimação de estado. Porém, para alcançar a mesma precisão dos métodos que aumentam o vetor de estado, exigem a realização de diversos ciclos de estimação de estado e parâmetros (ABUR; EXPÓSITO, 2004);

5. A opinião de (ZARCO; EXPÓSITO, 2000) é de que, para a estimação de parâmetros variáveis com o tempo, são mais apropriados os métodos que utilizam a teoria do Filtro de Kalman. Já os métodos que utilizam as Equações Normais são, por sua vez, mais atrativos para a estimação dos parâmetros que permanecem constantes, por um longo período de tempo;

6. A decisão referente aos parâmetros a serem estimados deve ser tomada considerando a qualidade e a redundância das medidas disponíveis, já que parâmetros sem erros podem perder a precisão se medidas com EGs forem utilizadas na sua estimação;

7. A seleção dos ramos suspeitos pode ser realizada através da análise de dados históricos;

8. Durante um determinado intervalo de tempo, os parâmetros série de linhas de transmissão não sofrem mudanças significativas de valor (ABUR; EXPOSITO, 2004);

9. Para estimar parâmetros que permanecem constantes durante um determinado intervalo de tempo, o melhor é realizar um procedimento off-line de estimação de 
parâmetros, através do processamento de medidas de várias amostras (em lote ou seqüencialmente);

10. A utilização de medidas de várias amostras possibilita o aumento da redundância das medidas, o que é um quesito de fundamental importância para a estimação de parâmetros;

11. Outra vantagem de proceder-se à estimação de parâmetros de maneira off-line é a possibilidade de pré-selecionar as informações que serão processadas, ou seja, serão selecionadas apenas amostras de medidas isentas de EGs e de erros de topologia.

Face ao exposto, diversos métodos foram desenvolvidos para validação de parâmetros de linhas de transmissão. Porém a maioria desses métodos preocupa apenas com os parâmetros série, considerando insignificante a influência dos erros nos parâmetros shunt das linhas de transmissão (CASTILLO et al., 2009a; CASTILLO et al., 2009b, DO COUTTO FILHO et al., 2008; LIU et al., 1992). Entretanto, vários trabalhos têm mostrado que essa influencia é significativa (MINGUEZ; CONEJO, 2007; LEFEBVRE et al., 2004; LEFEBVRE et al., 2006), principalmente em sistemas de transmissão com grande circulação (absorção e compensação) de potência reativa. Este é o caso do sistema de transmissão da Hydro-Québec TransÉnergie .

De acordo com (LEFEBVRE et al., 2008), a Hydro-Québec TransÉnergie utiliza o estimador de estado para produzir um caso base de fluxo de potência usado para análise de segurança da rede de transmissão, bem como, para considerações econômicas. Conseqüentemente, as quantidades estimadas devem ser tão próximas quanto possíveis dos valores "verdadeiros".

Para obter uma solução de estimação de estado mais confiável, vários estudos têm sido executados na Hydro-Québec (LEFEBVRE et al., 2004; LEFEBVRE et al., 2008). Porém a validação (detecção, identificação, e correção) de EPs dos ramos (admitância shunt e série) ainda representa um desafio.

\footnotetext{
5 TransÉnergie é uma divisão da Hydro-Québec.
} 


\subsection{Objetivos}

Pelo exposto, verifica-se a necessidade de se dispor de uma metodologia que permita depurar os dados referentes aos parâmetros de uma rede elétrica. Isto é, identificar parâmetros com valores incorretos, estimar valores mais confiáveis para estes parâmetros e, em seguida, validar estas estimativas.

Tomando por base os aspectos relevantes sobre o problema de EPs descritos na seção anterior, propõe-se uma metodologia que permita a depuração off-line de parâmetros série e shunt (condutância série, susceptância série e shunt) de linhas de transmissão, disponíveis nos bancos de dados das companhias de energia elétrica.

A metodologia proposta faz uso de medidas de diversas amostras e compreende três fases que possibilitam: identificação de parâmetros suspeitos de estarem com erro (Fase 1); estimação dos parâmetros suspeitos (Fase 2); e validação dos valores estimados (Fase 3).

Para detecção e identificação de parâmetros com erro (Fase1) calcula-se os resíduos normalizados das medidas através de um processo de estimação de estado convencional, por mínimos quadrados ponderados. A partir da análise destes resíduos, e do número de medidas incidentes para cada uma das linhas de transmissão do SEP, ou ramos do modelo barra-ramo, cria-se um Índice de Suspeita (IS) para cada um desses ramos e obtém-se uma lista de ramos suspeitos de estarem com erro.

Na Fase 2 estimam-se os parâmetros identificados como suspeitos na fase 1, ou seja, as condutâncias série e susceptâncias série e shunt dos ramos identificados como suspeitos. Para isso, propõe-se um estimador de estado e parâmetros baseado nas equações normais, que aumenta o vetor de variáveis de estado para inclusão dos parâmetros suspeitos. Em razão disto, temos agora um modelo aumentado e o vetor de variáveis de estado passa a ser chamado de vetor de variáveis de estado "aumentado".

A idéia que norteia a Fase 2 consiste em utilizar medidas de diversas amostras e estimar simultaneamente as variáveis de estado e os parâmetros suspeitos. Observe que, a partir da segunda amostra, os valores utilizados como condição inicial para os parâmetros suspeitos são as estimativas obtidas na amostra anterior, permitindo assim a correção dos valores desses parâmetros. 
Para melhorar o condicionamento numérico das matrizes envolvidas no processo de estimação do vetor de estado aumentado, bem como para preservar a redundância das medidas, o estimador de estado e parâmetros proposto divide o problema em dois subproblemas que são resolvidos simultâneamente a partir do algoritmo iterativo de Gauss - Newton. Ou seja, para cada amostra de medidas "k", para obter as correções das variáveis de estado convencionais ( $\Delta x_{e}^{k}$ ) são utilizados os valores atualizados dos parâmetros, e, para obter as correções dos parâmetros $\left(\Delta p^{k}\right)$ são utilizados os valores atualizados das variáveis de estado convencionais. O teste de convergência baseia-se na análise das alterações máximas em ambas as correções.

Em razão do efeito de espalhamento de resíduo, o processo de detecção e identificação de ramos suspeitos (Fase 1) pode falhar, isto é, pode classificar erroneamente um ramo como suspeito de estar com erro. Desta forma torna-se necessário validar as estimativas obtidas para os parâmetros suspeitos. Para isto, na Fase 3 utiliza-se um estimador de estado convencional, por mínimos quadrados ponderados, que é processado considerando os valores estimados na Fase 2, dos parâmetros dos ramos suspeitos, e não os valores disponíveis no banco de dados. Se a estimativa obtida na Fase 2 estiver próxima do valor "verdadeiro" do parâmetro de um determinado ramo suspeito, o correspondente IS diminuirá, validando as estimativas obtidas. Caso contrário, continuará o mesmo podendo, inclusive, aumentar.

Resultados de diversas simulações, com os sistemas de 14, 30 e 57 barras do IEEE, têm demonstrado a alta precisão e confiabilidade de metodologia proposta, mesmo na ocorrência de erros múltiplos (em mais de um parâmetro) em ramos adjacentes.

Para validar a metodologia proposta, a mesma foi aplicada em dois subsistemas da Hydro-Québec TransÉnergie, um deles consistindo de três linhas paralelas com compensação série. Os resultados obtidos foram bastante satisfatórios possibilitando, inclusive, uma atualização dos valores dos parâmetros disponíveis no banco de dados da companhia.

Finalizando, deve-se destacar que a metodologia proposta neste trabalho não considera medidas de PMU. Porém, tomando por base alguns estimadores de estado que fazem uso de medidas convencionais (medidas de potência ativa e reativa e de magnitudes 
de tensão) e de PMU propostos na literatura (ZHU; ABUR, 2007; LONDON et al., 2009), acreditamos que seja possível estender a metodologia aqui proposta para inclusão de PMU.

\subsection{Organização da tese}

Esta tese encontra-se dividida em 7 capítulos e 1 apêndice.

No Capítulo 2, apresenta-se a formulação matemática de alguns estimadores de estado aplicados em SEP e destacam-se as quatro etapas do processo de EESEP. Além disso, são discutidas algumas metodologias utilizadas na detecção e identificação de EGs e faz-se referência aos algoritmos mais utilizados.

No Capítulo 3, analisam-se as metodologias utilizadas na detecção e identificação de EPs, bem como os efeitos maléficos dos EPs para o processo de EESEP. Em seguida, apresenta-se uma ampla revisão bibliográfica sobre as diferentes metodologias desenvolvidas para o tratamento de EPs.

No Capítulo 4 é apresentada a metodologia proposta para depuração off-line de parâmetros série e shunt (condutância série e susceptâncias série e shunt) de linhas de transmissão. De forma complementar, expõe-se uma técnica para o "desacoplamento" da matriz jacobiana envolvida no problema de estimação de estado e parâmetros, que tem por objetivo melhorar o condicionamento numérico daquela matriz.

No Capítulo 5 discutem-se os resultados obtidos nos testes realizados nos sistemas IEEE, para comprovar a eficiência da metodologia proposta; e no capítulo 6 apresentam-se os resultados da aplicação da metodologia proposta em dois subsistemas elétricos da Hydro-Québec.

No Capítulo 7 são apresentadas as conclusões e algumas sugestões para a evolução do trabalho efetuado.

Apresenta-se, no Apêndice A, uma lista das publicações geradas a partir deste trabalho. No Apêndice B encontra-se um estudo das incertezas das medidas fornecidas ao estimador de estado, bem como a equação utilizada para calcular o desvio padrão das medidas utilizadas nos testes do capítulo 5 (os desvios padrão das medidas são utilizados para realizar a ponderação e para gerar os valores medidos das mesmas para efeito de simulação). No Apêndice C apresenta-se o método de newton e no Apêndice D é mostrado 
o número de condição da matriz Ganho. Por fim no Apêndice E apresenta-se a tecnica de escalonamento de linhas e colunas da matriz Ganho. 


\section{Capítulo 2}

\section{Estimação de Estado em Sistemas Elétricos de Potência}

\subsection{Introdução}

Em meados do século XX, mais precisamente em 1965, ocorreu o incidente que deixou sem alimentação de energia elétrica o nordeste dos Estados Unidos. As empresas de energia elétrica tomaram consciência de que deveriam realizar um grande esforço para desenvolver novas técnicas para operação dos SEP que permitiriam um elevado nível de segurança no fornecimento de energia elétrica. Isto mostrou que os antigos métodos e ferramentas para operação eram inadequados.

Começou a se falar de análise de segurança, índices de segurança, melhora da segurança, análise de estabilidade, otimização e começaram a se construir novos centros de controle. Até então, o controle e a decisão da operação se baseavam num sistema de supervisão, que controlava as posições dos dispositivos seccionadores (chaves e disjuntores) nas subestações, e um sistema separado, geralmente análogo ao anterior, que controlava de maneira automática a geração e o despacho econômico. Portanto, os únicos dados que o operador tinha disponíveis em tempo real eram o estado dos interruptores, a freqüência do sistema e o conjunto de medidas de potência necessário para o controle de geração.

Partindo desta situação, o esforço concentrou-se em conseguir, a cada intervalo de tempo, a informação, tanto dos dispositivos seccionadores como de todas as medidas do sistema que se controlava. Tendo todos estes valores em tempo real na base de dados, seria possível comprovar a segurança continuamente, isto é, poderia se analisar as condições de operação de cada equipamento da rede e detectar as situações anômalas e alarmantes de funcionamento. Este processo de captação, detecção e sinalização do sistema, juntamente 
com a utilização de telas gráficas e o armazenamento de todos os eventos, constituiu o sistema SCADA.

Pensou-se, inicialmente, que a partir de uma base de dados atualizada periodicamente pelo sistema SCADA, seria possível realizar a operação dos SEP de forma segura. Porém, tendo em vista o fato de as medidas obtidas pelo sistema SCADA apresentarem ruídos, inerentes do processo de medição, e a possibilidade de algumas medidas se tornarem momentaneamente indisponíveis, Schweppe verificou a necessidade de realizar-se uma "purificação" dos dados, antes de se tomar qualquer decisão relativa à operação dos SEP.

Ante o exposto, no final da década de 60 Schweppe publicou uma série de três artigos, apresentando a formulação geral do problema de EESEP (SCHWEPPE, 1970; SCHWEPPE; ROM, 1970; SCHWEPPE; WILDES, 1970; SCHWEPPE; HANDSCHIN, 1974). De acordo com Scheweppe, "o estimador de estado é um purificador de dados".

A partir dos trabalhos de Schweppe, o processo de EESEP vem sendo alvo de inúmeras pesquisas (DO COUTTO FILHO et al., 1990; MONTICELLI, 1999; ABUR; EXPÓSITO, 2004).

Nas próximas seções deste capítulo analisar-se-ão todas as etapas envolvidas no processo de EESEP. Será analisada, também, a utilização dos sistemas de medição fasorial sincronizada no processo de EESEP.

\subsection{Etapas do processo de EESEP}

Tradicionalmente, são quatro as etapas envolvidas no processo de EESEP:

Etapa 1: Obtenção da topologia do sistema, no modelo barra-ramo;

Etapa 2: Análise e restauração da Observabilidade do sistema;

Etapa 3: Estimação de estado propriamente dita;

Etapa 4: Processamento de Erros Grosseiros em Medidas Analógicas.

Como será apresentado nas próximas seções, as Etapas 2, 3 e 4 baseiam-se na topologia obtida na $1^{\text {a }}$ Etapa. Em razão disto, caso ocorra algum erro topológico e este não tenha sido detectado na etapa 1 , pelo configurador de redes, tal erro pode causar um aumento nos resíduos das medidas analógicas, localizadas nas vizinhanças dos elementos erroneamente configurados do sistema. Assim, na $4^{\text {a }}$ Etapa, as medidas analógicas, com resíduos elevados, serão identificadas como portadoras de EGs. Nessa situação, dar-se-á início a um processo de eliminação de medidas analógicas, e, eventualmente, o processo 
poderá reduzir a zero o nível de redundância local. Logo, não será mais detectado erro grosseiro em medida analógica, mas o erro topológico permanece. Conseqüentemente, o modelo do sistema não representará corretamente a sua atual situação.

Similar análise pode realizar-se, considerando erros nos parâmetros do sistema, pois, as Etapas 3 e 4 baseiam-se nos parâmetros fornecidos ao estimador, na $3^{\text {a }}$ Etapa. Assim, caso a informação de algum parâmetro do sistema tenha sido erroneamente fornecida ao estimador de estado, tal erro causará um aumento nos resíduos das medidas analógicas, localizadas nas vizinhanças do elemento, cujo parâmetro é o que forneceu aquela informação errada. Diante disto, vários autores realizaram então trabalhos, em busca de métodos para a análise de erros topológicos e de parâmetros (ZARCO; EXPÓSITO, 2000; ABUR; EXPÓSITO, 2004).

\subsubsection{Etapa 1: Obtenção da topologia do sistema, no modelo barra-ramo}

A partir das medidas lógicas, que são realizadas continuamente e consistem em estados de chaves e disjuntores, bem como de informações quanto ao tipo e à localização dos medidores instalados no sistema, o configurador de redes permite determinar a topologia e a correspondente configuração de medidores, no modelo barra-ramo (que corresponde ao diagrama unifilar do sistema) (PIERETI et al., 2007). Importa salientar que as informações processadas pelo configurador de sistemas são modeladas em nível de seção de barra (representação física dos elementos do sistema).

\subsubsection{Etapa 2: Análise e restauração da observabilidade do sistema}

Através do modelo barra-ramo, obtido pelo configurador de sistemas, verifica-se se é possível, através das medidas analógicas e virtuais ${ }^{6}$ disponíveis, determinar as variáveis de estado em todas as barras do sistema. Em caso afirmativo, o sistema é dito observável. Caso contrário, essa falta de medidas pode ser suprida, em algumas situações, por pseudomedidas ${ }^{7}$, através das quais o sistema se torna observável como um todo. Outra alternativa, para essa situação, é determinar as partes observáveis do sistema, isto é, as ilhas observáveis (QUINTANA et al., 1982; MONTICELLI et al., 1985a, 1985b; BRETAS, 1996;BENEDITO et al.,2008; LONDON et al., 2007; DE ALMEIDA et al., 2008).

\footnotetext{
${ }^{6}$ Medidas virtuais são medidas de injeção zero, em barras de passagem do sistema.

${ }^{7}$ Dados de previsão de carga, previsão de geração, dados históricos, etc. que fazem parte do banco de dados do centro de operação.
} 
Os métodos desenvolvidos para análise de observabilidade podem ser divididos, de uma forma geral, em dois grupos: os métodos topológicos e os numéricos.

Os métodos topológicos caracterizam-se pela criação de rotinas especificas que não exigem cálculos, mas que são de natureza combinatória e complexa (KRUMPPHOLZ et al., 1980; QUINTANA et al., 1982; NUCERA; GILLES, 1991). Já os métodos numéricos são mais simples, visando à utilização de rotinas já disponíveis nos programas de estimadores de estado. Entretanto, estão sujeitos a erros numéricos (MONTICELLI; WU 1985b; MONTICELLI; WU, 1985a; MONTICELLI; WU, 1986).

Verificando a possibilidade de diminuir a quantidade de cálculos necessária para a análise de observabilidade, (BRETAS, 1996) desenvolveu um novo método baseado na fatoração triangular da matriz ganho do estimador por mínimos quadrados ponderados e nos conceitos contidos nos caminhos de grafo. Dando continuidade a essa linha de pesquisa, em ( et al., 2008) foi desenvolvido um método para análise de observabilidade baseado na fatoração triangular da matriz jacobiana do estimador por mínimos quadrados ponderado e nos conceitos contidos nos caminhos de grafo.

\subsubsection{Etapa 3: Estimação de Estado}

Considerando a topologia do sistema, obtida pelo configurador de sistemas, e através dos seus parâmetros armazenados no banco de dados, bem como do conjunto disponível de medidas, o estimador de estado permite determinar as variáveis de estado de todas as barras do sistema.

O estimador de estado pode ser estático ou dinâmico, sendo o primeiro abordado neste trabalho, podendo ser considerado uma generalização do problema clássico de fluxo de carga (HANDASCHIN et al., 1975). Já o estimador dinâmico, considera as relações entre as grandezas em instantes de tempo diferentes.

Em (DO COUTTO FILHO; SOUZA, 2009) apresenta-se uma revisão bibliográfica sobre estimadores de estado que utilizam informações obtidas previamente, conhecidos de uma forma geral como estimadores dinâmicos de estado ou estimadores FASE (do inglês, Forecasting-Aided State Estimators).

O estimador de estado calcula as variáveis de estado desconhecidas, através de um conjunto de medidas não exatas. Logo, o resultado obtido para as variáveis de estado desconhecidas também não será exato. 
Dessa forma, o problema de estimação de estado consiste em encontrar uma maneira de atingir-se a melhor estimativa das variáveis de estado desconhecidas. Para isto, dos muitos métodos existentes, o mais utilizado em SEP é o método dos Mínimos Quadrados Ponderados (ABUR; EXPOSITO, 2004).

A estimação de estado, através dos mínimos quadrados, formula-se considerando:

$$
\underline{z}=h\left(\underline{x}_{v}\right)+\underline{w}
$$

Onde: $\underline{z}$ - vetor de medidas $(\mathrm{mx} 1) ; \underline{h}($.$) - vetor de funções não lineares, relacionando$ as medidas com as variáveis de estado $(\mathrm{mx} 1) ; \underline{x}_{v}$ - vetor das variáveis de estado verdadeiras $(\mathrm{nx} 1) ; \underline{w}$ - vetor dos erros das medidas $(\mathrm{mx} 1) ; \mathrm{m}$ - número de medidas; $\mathrm{n}$ - número de variáveis de estado a serem estimadas.

A melhor estimativa do vetor $\underline{x}_{v}$, designada por $\underline{\hat{x}}$, é o valor de $\underline{x}$ que torna mínimo o índice $J(\underline{x})$, dado por:

$$
J(\underline{x})=\underline{w}^{t} W \underline{w}
$$

$\mathrm{ou}$

$$
J(\underline{x})=[\underline{z}-h(\underline{x})]^{t} W[\underline{z}-h(\underline{x})]
$$

Onde $W$ é uma matriz de ponderação para as medidas; é o inverso da matriz covariância das mesmas. É uma matriz diagonal, cujos valores diferentes de zero são os inversos das variâncias de cada medida $\left(\sigma_{i i}^{-1}\right)^{2}$ (HANDSCHIN et al., 1975; HORISBERGER et al., 1976). Através dessa matriz, as medidas são ponderadas conforme as suas qualidades.

Da equação (2.3) deduz-se que $J(\underline{x})$ é uma função quadrática. Considerando que $\underline{x}_{v}$ torna mínimo $J(\underline{x})$, podemos dizer que $J(\underline{x})$ é convexo nas proximidades de $\underline{x}_{v}$. Desta forma, para obter $\underline{\hat{x}}$, que torne $J(\underline{x})$ mínimo, fazemos:

$$
\frac{\partial J(\underline{x})}{\partial \underline{x}}=0
$$

portanto

$$
2 H(\underline{\hat{x}})^{t} W[\underline{z}-h(\underline{\hat{x}})]=0
$$


onde $H(\underline{\hat{x}})$ é a matriz Jacobiana, dada por:

$$
\left.H(\underline{\hat{x}}) \underset{=}{\Delta \frac{\partial h(\underline{x})}{\partial \underline{x}}}\right|_{\underline{\hat{x}}}
$$

Como podemos ver, a solução da equação (2.5) fornece o vetor $\underline{\hat{x}}$. Considerando a não linearidade de $H(\hat{x})$ e $h(\underline{\hat{x}})$, a solução direta dessa equação não é possível. Assim, o vetor estimado $\underline{\hat{x}}$ é obtido através de técnicas iterativas. Para isso, utiliza-se o método de Newton-Raphson.

Expandindo em série de Taylor a equação $\mathrm{h}(\underline{\mathrm{x}})$, e tomando apenas os termos lineares, em torno de um ponto inicial $\underline{x}^{0}$, têm-se:

$\underline{h}(\underline{x}) \cong \underline{h}\left(\underline{x}^{0}\right)+H\left(\underline{x}^{0}\right) \Delta \underline{x}^{0}$

Sendo: $\Delta \underline{x}^{0}=\underline{x}-\underline{x}^{0}$

De (2.1) obtêm-se:

$$
\underline{z}=\underline{h}\left(\underline{x}^{0}\right)+H\left(\underline{x}^{0}\right) \Delta \underline{x}^{0}+\underline{w}
$$

Define-se:

$$
\Delta \underline{z}\left(\underline{x}^{0}\right)=\underline{z}-\underline{h}\left(\underline{x}^{0}\right)
$$

Onde $\Delta \underline{z}$ é o erro de estimação, obtendo-se:

$$
\Delta \underline{z}\left(\underline{x}^{0}\right)=H(\underline{x})^{0} \Delta \underline{x}^{0}+\underline{w}
$$

Assim, a função objetivo passa a ser: 


$$
J(\underline{x})=\left[\Delta \underline{z}\left(\underline{x}^{0}\right)-H\left(\underline{x}^{0}\right) \Delta \underline{x}^{0}\right]^{t} W\left[\Delta \underline{z}\left(\underline{x}^{0}\right)-H\left(\underline{x}^{0}\right) \Delta \underline{x}^{0}\right]
$$

E o mínimo é encontrado fazendo-se:

$$
H\left(\underline{x}^{0}\right)^{t} W\left[\Delta \underline{z}\left(\underline{x}^{0}\right)-H\left(\underline{x}^{0}\right) \Delta \underline{x}^{0}\right]=0
$$

Portanto:

$$
\Delta \underline{x}^{0}=\left[H\left(\underline{x}^{0}\right)^{t} W H\left(\underline{x}^{0}\right)\right]^{-1} H\left(\underline{x}^{0}\right)^{t} W \Delta \underline{z}\left(\underline{x}^{0}\right)
$$

Onde a matriz ganho é dada por:

$$
G\left(\underline{x}^{0}\right)=\left[H\left(\underline{x}^{0}\right)^{t} W H\left(\underline{x}^{0}\right)\right]
$$

$\mathrm{e}$

$$
\underline{x}^{1}=\underline{x}^{0}+\Delta \underline{x}^{0}
$$

Assim, a estimativa de $\underline{x}_{\mathrm{v}}$ corresponde ao valor de $\underline{\mathrm{x}}$, para uma determinada iteração, em que se verifique um índice de convergência pré fixado.

\subsubsection{Algoritmo do Estimador de Estado Acoplado por Mínimos Quadrados Ponderados}

Passo 1: Fazer $v=0$ e escolher uma solução inicial $\underline{x}^{v}=\underline{x}^{0}$;

Passo 2: Calcular as matrizes $H\left(\underline{x}^{v}\right)$ e $G\left(\underline{x}^{v}\right)$ no ponto $\underline{x}=\underline{x}^{v}$;

Passo 3: Obter a correção nas variáveis de estado através da equação normal e atualizar as variáveis:

$$
\begin{gathered}
\Delta \underline{x}^{v}=G\left(\underline{x}^{v}\right)^{-1} \cdot H\left(\underline{x}^{v}\right)^{t} \cdot R^{-1} \cdot \Delta \underline{z}\left(\underline{x}^{v}\right) \\
\underline{x}^{v+1}=\underline{x}^{v}+\Delta \underline{x}^{v}
\end{gathered}
$$

Passo 4: Testar a convergência: se $\max \left|\Delta \underline{x}^{v}\right| \leq \mathcal{\varepsilon}$, o processo convergiu. Caso contrário, faça $v=v+1_{\mathrm{e}}$ volte ao passo 2 . 
O maior esforço computacional envolvendo o algoritmo acoplado do processo de estimação de estado por WLS apresentados anteriormente está associado ao cálculo da matriz $\mathrm{G}$ e, posteriormente, à sua fatoração a cada iteração. Assim como no problema de fluxo de carga, uma alternativa encontrada para reduzir os cálculos envolvidos no processo de estimação de estado é manter constante, mas aproximada, a matriz Jacobiana que relaciona as equações das medidas com as variáveis de estado.

Para tanto, com base no princípio de que as potências ativas são influenciadas predominantemente pelos ângulos de fase de tensão, e de que as potências reativas são influenciadas predominantemente pelas magnitudes de tensão, várias versões de estimadores de estado desacoplados e desacoplados rápidos foram propostas na literatura (HORISBERGER et al., 1976; ASCHMONEIT et al., 1977; HORTON; MASIELLO, 1977; GARCIA et al., 1979; ALLEMONG et al., 1982; MONTICELLI; GARCIA, 1990). Dentre estas, destaca-se a versão desenvolvida por (MONTICELLI; GARCIA, 1990), em razão desta chegar a resultados satisfatórios, mesmo em sistemas com relação $x / r$ baixa. Dada esta vantagem, o mesmo é apresentado a seguir.

\subsubsection{Formulação do Estimador por Mínimos Quadrados Ponderados Desacoplado Rápido}

Considere a seguinte formulação desacoplada, para o sistema de equações nãolineares que representam o problema de estimação de estado em sistemas elétricos:

$$
\begin{aligned}
& h_{p}(\underline{x}) \cong \underline{z}_{p}, \\
& h_{q}(\underline{x}) \cong \underline{z}_{q},
\end{aligned}
$$

sendo:

- $p$ o sub-índice que representa o conjunto ativo, formado por medidas de injeção e fluxo de potência ativa;

- $q$ o sub-índice que representa o conjunto reativo, formado por medidas de injeção e fluxo de potência reativa, além das medidas de magnitude de tensão elétrica;

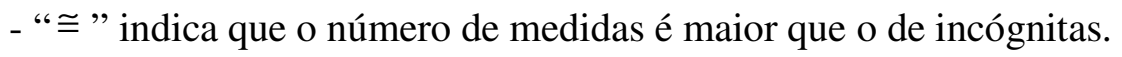

O vetor de variáveis de estado $\underline{x}$ é decomposto da seguinte forma: 


$$
\underline{x}=\left[\begin{array}{l}
\underline{\theta} \\
\underline{V}
\end{array}\right],
$$

onde $\underline{\mathrm{V}}$ é o vetor de magnitudes de tensão elétrica $(n b \times 1)$ e $\underline{\theta}$ é o vetor de ângulos de tensão elétrica $(n b \times 1)$, sendo nb o número de barras do SEP.

A partir da divisão do conjunto de medidas e da decomposição do vetor de variáveis de estado, pode-se decompor a matriz Jacobiana da seguinte forma:

$$
H(x)=\frac{\partial h(x)}{\partial x}=\left[\begin{array}{ll}
\frac{\partial h_{p}(\theta)}{\partial \theta} & \frac{\partial h_{p}(V)}{\partial V} \\
\frac{\partial h_{q}(\theta)}{\partial \theta} & \frac{\partial h_{q}(V)}{\partial V}
\end{array}\right] \stackrel{\Delta}{=}\left[\begin{array}{cc}
H_{p \theta} & H_{p V} \\
H_{q \theta} & H_{q V}
\end{array}\right]
$$

A solução ótima do vetor $\underline{x}$, através do estimador WLS desacoplado rápido (MONTICELLI; GARCIA, 1990), é obtida através de um procedimento iterativo, cujas correções $\Delta \underline{\theta}$ e $\Delta \underline{V}$ são calculadas separadamente. Nessa abordagem os efeitos das submatrizes $\mathrm{H}_{\mathrm{pV}}$ e $\mathrm{H}_{\mathrm{q} \theta}$ são totalmente desprezados. Veja o algoritmo a seguir.

\subsubsection{Algoritmo Desacoplado Rápido ( ${ }^{8}$ (versão “primal” ou $B X$ )}

As sub-matrizes $H_{p \theta}$ e $H_{q V}$ são consideradas fixas ao longo das iterações, sendo as mesmas calculadas com $\underline{\theta}=\underline{0}^{\circ}, \underline{V}=\underline{1} p u$ ("flat start") e desprezando as resistências série na formação da sub-matriz $H_{q V}$. A partir dessas considerações, a solução ótima por mínimos quadrados ponderados pode ser obtida através de dois passos (MONTICELLI; GARCIA, 1990):

i) Cálculo da iteração ativa $P \theta$ :

$$
\begin{gathered}
\Delta \underline{\theta}^{v}=\left(H_{p \theta}^{t} \cdot R_{p}^{-1} \cdot H_{p \theta}\right)^{-1} \cdot H_{p \theta}^{t} \cdot R_{p}^{-1} \cdot\left[\underline{z}_{p}-h_{p}\left(\underline{\theta}^{v}, \underline{V}^{v}\right)\right], \\
\underline{\theta}^{v+1}=\underline{\theta}^{v}+\Delta \underline{\theta}^{v} .
\end{gathered}
$$

ii) Cálculo da iteração reativa $Q V$ :

\footnotetext{
${ }^{8}$ Em Monticelli e Garcia (1990) e Monticelli (1999), o algoritmo desacoplado rápido é apresentado tanto em versão "primal", quanto em versão "dual" ou $X B$, a qual não será abordada neste trabalho.
} 


$$
\begin{gathered}
\Delta \underline{V}^{v}=\left(H_{q V}^{t} \cdot R_{q}^{-1} \cdot H_{q V}\right)^{-1} \cdot H_{q V}^{t} \cdot R_{q}^{-1} \cdot\left[\underline{z}_{q}-h_{q}\left(\underline{\theta}^{v+1}, \underline{V}^{v}\right)\right], \\
\underline{V}^{k+1}=\underline{V}^{k}+\Delta \underline{V}^{k} .
\end{gathered}
$$

O processo iterativo encerra-se quando as magnitudes das correções $\Delta \theta$ e $\Delta V$ forem menores que os critérios de parada adotados, por exemplo, quando

$$
\begin{gathered}
\max \left|\Delta \underline{\theta}_{i}\right| \leq \varepsilon_{p \theta, \mathrm{e}} \\
\max \left|\Delta \underline{V}_{i}\right| \leq \varepsilon_{q V},
\end{gathered}
$$

no qual $\varepsilon_{\mathrm{p} \theta}$ e $\varepsilon_{\mathrm{qV}}$ denotam, respectivamente, a tolerância de parada do modelo ativo e do modelo reativo. Ademais, quando uma das tolerâncias é alcançada, fixa-se o respectivo vetor de estado e processa-se apenas a meia-iteração, na qual a tolerância não foi obtida. Conseqüentemente, o processo torna-se ainda mais rápido.

A solução do problema de estimação de estado pelo método da equação normal tornase deficiente em grandes sistemas de potência (SILVA GOUVÊA; SIMÕES COSTA, 1998). O principal problema dessa solução é a instabilidade numérica. Uma vez que a solução do problema de estimação de estado é iterativa, o mau condicionamento do sistema acarreta em uma convergência lenta, ou pior, a não-convergência.

Análises feitas sobre o método da equação normal demonstram que os principais fatores para o mau condicionamento do método são:

- Disparidade nos fatores de ponderação (desvio padrão) entre as diversas medidas (ASCHMONEIT et al., 1977);

- Número elevado de medidas de injeção (GU et al., 1983);

- Conexões entre linhas curtas e longas (MONTICELLI et al., 1985).

$\mathrm{Na}$ literatura, encontram-se diversos métodos desenvolvidos para solucionar esse problema de instabilidade numérica e mau condicionamento. As principais metodologias são:

- Equações normais com restrições de igualdade (ASCHMONEIT et al., 1977);

- Método de Peter e Wilkinson (GU et al., 1983);

- Método híbrido (MONTICELLI et al., 1985);

- Transformação ortogonal (SIMÕES COSTA; QUINTANA, 1981); 
- Método da matriz aumentada de Hatchel (GJELSKI et al., 1985).

Em (ALVARADO; TINNEY, 1990) revisaram-se os principais métodos de solução do problema de estimação de estado e em (HOLTEN et al., 1988) comparam-se os seguintes métodos apresentando as vantagens e desvantagens de cada um deles.

- Método de equações normais convencionais;

- Método de equações normais com restrições;

- Método de transformações ortogonais;

- Método híbrido;

- Método da matriz aumentada de Hatchel;

Do ponto de vista de:

- Estabilidade numérica;

- Eficiência computacional;

- Complexidade na realização.

No artigo chegou-se na conclusão de que o método híbrido e o de Hatchel são os que oferecem melhores compromissos entre estabilidade numérica e eficiência computacional, já que, embora o método de transformações ortogonais seja numericamente mais estável, não se pode realizar com o método desacoplado rápido.

\subsubsection{Etapa 4: Processamento de Erros Grosseiros em Medidas Analógicas}

Como mencionado no Capítulo 1, as medidas analógicas, fornecidas ao estimador de estado, estão sujeitas aos EGs ${ }^{9}$. Na prática, esses erros são causados, por exemplo, por problemas nos canais de comunicação, instrumentos de medição defeituosos, erro na modelagem de pseudo-medidas, etc.

Devido a essa fragilidade do conjunto de medidas, o estimador de estado deve ser robusto o suficiente para detectar e identificar a ocorrência de EGs. As medidas identificadas como portadoras de EGs são eliminadas e as variáveis de estado estimadas novamente.

Dentre os diversos métodos desenvolvidos, para detecção e identificação de medidas com EGs, os mais utilizados são aqueles baseados na análise estatística dos resíduos das

\footnotetext{
${ }^{9}$ Medidas portadoras de EGs são aquelas com grau de imprecisão muito maior do que é suposto pelo modelo de medição.
} 
medidas que é a diferença entre entre o valor medido e o valor estimado das medidas (MONTICELLI, 1999; ABUR; EXPOSITO, 2004).

A detecção de medidas com EGs pode ser feita através do índice $J(\underline{\hat{x}})$. Considerando a hipótese de que não haja erro grosseiro, o valor do índice $J(\underline{\hat{x}})$, calculado para $\underline{\hat{x}}$ obtido após a convergência do processo de estimação de estado, é comparado com o parâmetro $\lambda$. $\mathrm{O}$ valor de $\lambda$ é previamente determinado, supondo uma distribuição $\chi^{2}$, com $(m-n)$ graus de liberdade ${ }^{10}$ para o índice $J(\underline{\hat{x}})$ e fixada uma certa probabilidade $\rho$ de se tomar a decisão errada, rejeitando-se a hipótese quando ela for verdadeira.

Se $J(\underline{\hat{x}})>\lambda$, rejeita-se a hipótese de que não haja erro grosseiro, e, se $J(\underline{\hat{x}})<\lambda$ aceita-se a mesma.

Se a hipótese de que não haja erro grosseiro for aceita, consideram-se confiáveis os resultados obtidos pelo estimador de estado. Mas, se essa hipótese for rejeitada, importa identificar e eliminar as medidas que estejam com EGs.

Os métodos mais empregados, para identificação de medidas com EGs, têm sido aqueles que se baseiam na análise estatística dos resíduos de estimação normalizados. O vetor resíduo de estimação pode ser definido como:

$$
\underline{r}=\underline{z}-h(\underline{\hat{x}})
$$

Que pode ser representado também da seguinte forma (HANDSCHIN et al., 1975):

$$
\underline{r}=\Gamma \underline{w}
$$

Onde:

$\underline{w}$ - é o vetor aleatório dos erros das medidas;

$\Gamma$ - é a matriz sensibilidade do resíduo, dada por:

$$
\Gamma=\mathrm{I}-\mathrm{H}(\underline{\hat{\mathrm{x}}})\left[\mathrm{H}(\underline{\hat{\mathrm{x}}})^{\mathrm{t}} \mathrm{WH}(\underline{\hat{\mathrm{x}}})\right]^{-1} \mathrm{H}(\underline{\hat{\mathrm{x}}})
$$

Sendo $I$ uma matriz identidade.

A matriz covariância do vetor $\underline{r}$ é a matriz $R$ dada por:

\footnotetext{
${ }^{10}$ Lembrando que $\mathrm{m}$ e $\mathrm{n}$ representam, respectivamente, o número de medidas e o número de variáveis de estado de um sistema de potência.
} 


$$
R=W-H(\underline{\hat{x}})\left[H(\underline{\hat{x}})^{t} W^{-1} H(\underline{\hat{x}})\right]^{-1} H(\underline{\hat{x}})^{t}
$$

Considerando $\rho_{i i}$ o elemento (i,i) da matriz $R$, os resíduos normalizados $\tau_{i}$ ficam definidos como:

$$
\tau_{i}=\frac{r_{i}}{\sqrt{\rho_{i i}}}
$$

com $\mathrm{i}=1,2, \ldots \mathrm{m}$.

Onde $\tau_{i}$ é, aproximadamente, uma distribuição normal de média $\bar{\tau}_{i}$, dada por:

$$
\bar{\tau}_{i}=\frac{\bar{r}_{i}}{\sqrt{\rho_{i i}}}
$$

e desvio padrão unitário.

Quando for detectada a presença de medidas com EGs, é acrescentada à equação (2.1) um vetor determinístico b, para representar os EGs. Assim a equação (2.1) toma a seguinte forma:

$$
\underline{Z}=h\left(\underline{x_{v}}\right)+\underline{w}+\underline{b}
$$

Considerando que apenas a medida $j$ possua $\mathrm{EG}, \mathrm{o}$ vetor $\underline{b}$ será dado por:

$$
\underline{b}=\left[\begin{array}{c}
0 \\
\cdot \\
\cdot \\
j \\
\cdot \\
\cdot \\
0
\end{array}\right], \quad \text { sendo } b_{j} \text { o erro grosseiro da medida } j
$$

Assim, a média do resíduo de estimação é:

$$
\underline{\underline{r}}=\Gamma \underline{b}=\left[\begin{array}{c}
\gamma_{1 j} \\
\cdot \\
\cdot \\
\gamma_{i j} \\
\cdot \\
\cdot \\
\gamma_{m j}
\end{array}\right] \cdot b j \Rightarrow \bar{r}_{i}=b_{j} \cdot \gamma_{i j} \quad, \quad \mathrm{i}=1,2,3, \ldots, \mathrm{m} .
$$

onde $\gamma_{i j}$ é o elemento (i,j) da matriz $\Gamma$, que é obtida pela equação (2.28).

Através da equação (2.30) chegamos à expressão: 


$$
\bar{\tau}_{i}=\frac{b_{j} \cdot \gamma_{i j}}{\sqrt{\rho_{i i}}}, \quad \mathrm{i}=1,2, \ldots, \mathrm{m}
$$

Entretanto, para cada medida, somente um $\rho_{i i}$ é encontrado. Desta forma, as médias dos resíduos normalizados de cada medida são diferentes, mas com variâncias iguais e unitárias. Portanto, as distribuições de probabilidade dos resíduos normalizados, de cada medida, diferem apenas no que se refere às médias. Conseqüentemente, para a identificação de medidas com EGs, basta examinar as médias dos resíduos normalizados de cada medida. A medida que tiver o $\bar{\tau}_{i}$ mais distante das demais, ou seja, a medida que tiver o maior resíduo normalizado corresponderá à medida com erro grosseiro (SCHWEPPE, 1970; HANDSCHIN et al., 1975).

Então, sendo $\tau_{i}^{\max }$ o maior resíduo normalizado, se $\tau_{i}^{\max }>\alpha$, a correspondente medida "i" é portadora de erro grosseiro ( $\alpha$ é o limite de identificação e depende de níveis de probabilidade aceitáveis de falso-alarme e de não identificação (MONTICELLI, 2000).

Após identificar a medida portadora de EG é necessário eliminar o seu efeito sobre a estimativa. Isso pode ser feito de duas formas:

2 Eliminando a medida com EG do conjunto de medidas e proceder-se à estimação de estado novamente;

ii) Recuperando o valor da medida com EG, através do valor de erro estimado, conhecido como método $\underline{\hat{b}}$ proposto em (MONTICELLI; GARCIA, 1983). Em seguida realiza-se o processo de estimação de estado novamente.

O processo de estimação de estado como um todo termina quando, após uma estimativa, não for detectada/identificada nenhuma medida com EG.

Vale destacar que o nível de redundância das medidas afeta a capacidade de se detectar e identificar possíveis EGs. Assim, apresentar-se-á, a seguir, o conceito de medida crítica e conjunto crítico de medidas.

\subsubsection{Medida Crítica}

Uma medida é considerada crítica, quando a retirada dessa medida, de um sistema observável, torna-o não-observável. Além disso, é impossível detectar EG em tais medidas, já que apresentam resíduo nulo (CLEMENTS et al., 1981). 
As medidas não críticas são consideradas como redundantes e somente essas medidas têm resíduos não nulos (CLEMENTS et al., 1981). Entretanto, as medidas redundantes podem ou não pertencer a algum conjunto crítico de medidas (veja o conceito a seguir).

\subsubsection{Conjunto Crítico de Medidas}

Conjunto crítico de medidas, também conhecido na literatura como "minimally dependent sets of measurements", ou "bad data groups", pode ser definido segundo (AYRES; HALEY, 1986) de duas formas:

Definição numérica: os conjuntos críticos de medidas são aqueles correspondentes às submatrizes da matriz covariância dos resíduos (equação 3.18), com posto igual a 1;

Definição topológica: conjunto crítico de medidas é o conjunto de medidas formado por medidas redundantes (que não são críticas), em que a eliminação de uma medida qualquer, a ele pertencente, torna as demais medidas críticas.

A identificação dos conjuntos críticos é importante para um desempenho confiável do estimador de estado (SIMÕES-COSTA et al., 1990). Isto porque, além de esses conjuntos críticos representarem um risco à observabilidade de um sistema elétrico, a identificação de EGs em medidas pertencentes a um conjunto crítico é impossível, pois seus resíduos normalizados apresentam valores iguais em módulo (MILI et al., 1984).

Face ao exposto, diversos métodos foram desenvolvidos para identificação de medidas críticas e de conjuntos críticos de medidas (SIMÕES-COSTA et al., 1990; DO COUTTO FILHO et al., 2001; KORRES; CONTAXIS, 1991a, 1991b; LONDON et al., 2007).

\subsection{Sistema de Medição Fasorial Sincronizada}

Uma das causas dos EGs é a falta de sincronismo dos dados obtidos pelo sistema SCADA, isto ocorre pela ausência de uma fonte de sincronização das medições realizadas no SEP.

Nas duas últimas décadas, entretanto, vários trabalhos de pesquisa têm abordado a possibilidade da obtenção de um sistema de medição fasorial sincronizada (SPMS do inglês, Synchronized Phasor Measurement Systems) (THORP et al., 1985; PHADKE et al., 1986; PHADKE, 1993) e, atualmente, a implantação de equipamentos responsáveis por essas aferições, as Unidades de Medição Fasorial - (PMUs do inglês, "Phasor Measurement Units"), já é uma realidade que se apresenta como uma forte tendência de utilização. 
Face ao exposto, diversas pesquisas vêm sendo realizadas objetivando a inclusão das medidas fasoriais para melhoria do processo de EESEP.

O SPMS constitui-se, basicamente, dos seguintes elementos: PMU, que é o elemento base de todo o sistema; estações de recepção do sinal de GPS; concentrador de dados; e canais de comunicação (veja Figura 2.1).

As funções da PMU são: aquisição das tensões e correntes nas barras do SEP; processamento e formatação dessas informações e envio ao concentrador de dados. $\mathrm{O}$ concentrador de dados tem como principal função receber e organizar as medidas fasoriais enviadas pelas PMUs, disponibilizando-as para uso em diversas aplicações (como por exemplo, estimação de estado). Os canais de comunicação, por sua vez, têm a função de viabilizar a transferência de dados entre as PMUs e o concentrador de dados. Atualmente, os canais de comunicação considerados para uso em SMSF incluem opções de estruturas que operam tanto através de meios físicos específicos (linhas telefônicas, cabos de fibra ótica ou mesmo a rede de baixa ou média tensão), quanto através de tecnologia sem fio, amplamente denominada wireless (canais de microondas ou de sistemas de satélites).

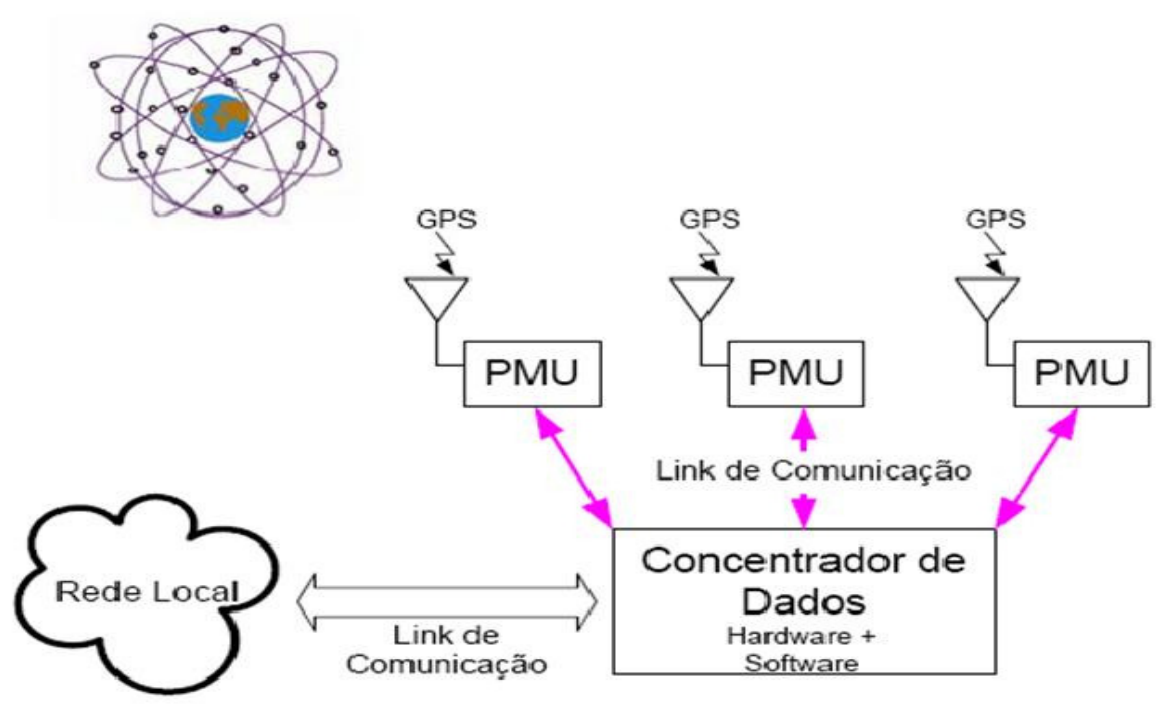

Figura 2.1: Visão geral de um SPMS (EHRENSPERGER, 2004)

\subsubsection{Unidades de medição fasorial - PMUs}

A PMU é um instrumento de medição desenvolvido em meados da década de 80 (ZHOU et al., 2006) e realiza: aquisição das tensões e correntes nas barras do sistema; 
processamento dos dados amostrados, efetuando assim a medição das grandezas fasoriais; para, em seguida, enviá-las ao concentrador de dados.

A Figura 2.2 ilustra a estrutura simplificada de uma PMU, que consiste basicamente de um sistema de aquisição de dados [onde se encontram os filtros anti-aliasing, o circuito oscilador (phase-locked oscillator) e o módulo de conversão analógica/digital] e de um microprocessador que realiza o tratamento matemático das amostras.

Cada PMU deve estar acoplada a um equipamento receptor de sinal de GPS. Essa estrutura foi proposta por (PHADKE et al., 1993) e tem se mantido ao longo dos anos.

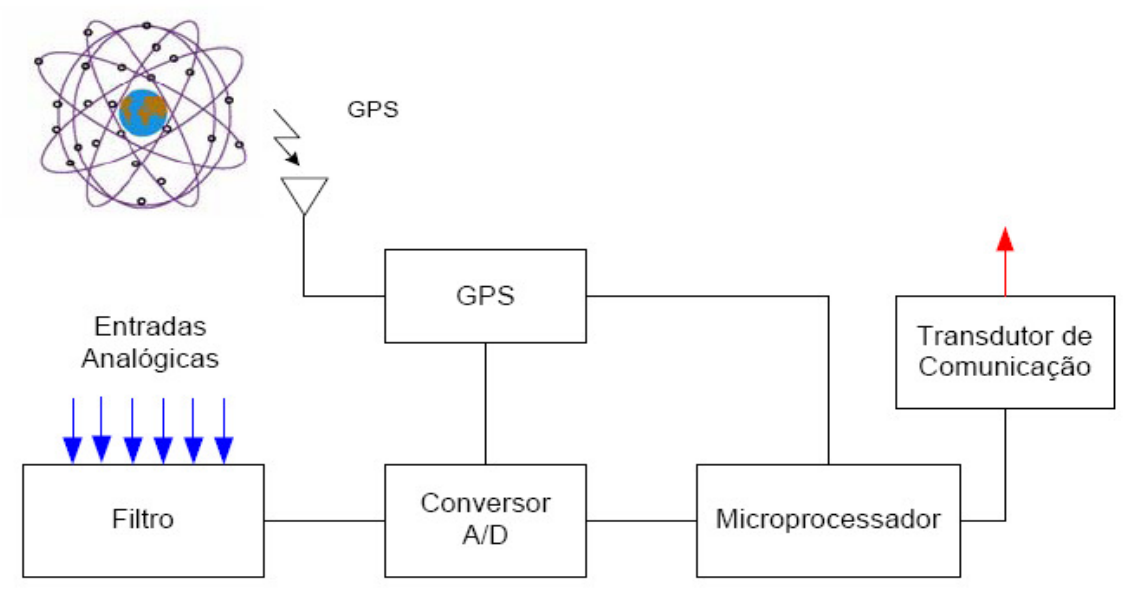

Figura 2.2: Estrutura básica de uma PMU (EHRENSPERGER, 2004)

\section{Processo de Medicão Fasorial}

Em razão de as PMUs utilizarem uma fonte eficaz de sincronização, fornecida pelo sistema GPS, elas viabilizam a realização da medição de grandezas fasoriais a uma taxa de até 60 medidas por segundo e com precisão angular adequada aos requisitos da maioria das aplicações de monitoração e controle em tempo real (EHRENSPERGER, 2004).

Continuamente, o sistema GPS envia sinal de um pulso por segundo às estações receptoras. Considerando que esse trem de pulsos é enviado com precisão maior do que $1 \mu \mathrm{s}$ e que pode ser recebido por estações distantes entre si com igual precisão, é possível a sincronização das amostras obtidas por diversas PMUs, instaladas em diferentes pontos de um SEP.

As PMUs disponíveis atualmente têm a capacidade de medir fasores de tensão, corrente e potência, além da freqüência e variação da freqüência no tempo. Os dados medidos são exteriorizados em grandezas de fase ou grandezas de seqüência positiva, em forma polar 
ou retangular. Em seguida, esses dados são transferidos, através de canais de comunicação, ao concentrador de dados, utilizando um formato de dados padronizado.

O processo de cálculo dos fasores é um ponto chave do desempenho das PMUs, normalmente utiliza-se a Transformada Discreta de Fourier (PHADKE, 1993).

Vale lembrar que o fasor é uma representação matemática de uma forma de onda senoidal, tomando como referência, para a determinação do ângulo de fase, o instante do início da amostragem e considerando a freqüência constante. O conceito de fasor com freqüência variável, como ocorre na prática, não é claramente definido e pode gerar diferentes interpretações. Para contornar tal problema, normas definem limites de erros permissíveis, em condições limites, em termos de variação da freqüência e magnitude do sinal medido.

\subsubsection{Aplicação em Estimação de Estado}

Tendo em vista a sincronização dos dados no tempo, a possibilidade de dispor-se de medições sincronizadas ao longo de um SEP de grandes dimensões e a elevada freqüência com que os dados são apresentados, acredita-se que a medição proporcionada pelas PMUs é de qualidade bem superior àquela obtida através do sistema SCADA convencional (medidas de potência, magnitude de tensão e injeção de corrente).

Face ao exposto, vêm sendo apresentadas, nos últimos anos, diversas propostas para a inclusão das medidas fasoriais, visando à melhoria do processo de EESEP.

Em trabalhos como aqueles de (THORP et al., 1985; PHADKE et al., 1986; ZIVANOVIC; CAIRNS, 1996; ZHOU et al., 2006), demonstra-se-nos que, quando as medidas fasoriais são adicionadas às medidas convencionais, no processo de EESEP, a precisão deste é aumentada. Além disto, desde que todas as tensões de barra de um sistema sejam monitoradas por PMUs, o problema de estimação pode ser resolvido utilizando somente medidas fasoriais (PHADKE, 2002). Neste caso o problema tornar-se-á linear, já que as medidas relacionarão diretamente as variáveis de estado, ao contrário das medidas convencionais de potência, as quais relacionam medidas e variáveis, através de funções nãolineares.

Entretanto, a maioria dos SEP ainda não se torna observável, considerando apenas as PMUs. Além disto, resultados de alguns estudos indicam que a utilização em conjunto de medidas convencionais com medidas fasoriais sincronizadas propicia mais eficiência ao 
processo de filtragem de erros, do que o uso apenas de medidas fasoriais sincronizadas (ZIVANOVIC; CAIRNS, 1996; BRUNO et al., 2009).

O desenvolvimento de metodologias, para o tratamento de questões relacionadas à observabilidade e redundância das medidas, é outro ponto de muita relevância nesse contexto, o qual vem sendo alvo de várias pesquisas (ZHOU et al., 2008; LONDON et al., 2009). 


\section{Capítulo 3}

\section{Erros de Parâmetros no Processo de Estimação de Estado em Sistemas Elétricos de Potência}

\subsection{Influência de erros de parâmetros no processo de Estimação de Estado em Sistemas Elétricos de Potência}

Para a realização do processo de EESEP, comumente se supõe que os parâmetros usados nos modelos, que representam os elementos da rede elétrica, sejam perfeitamente conhecidos, isto é, não possuem erros. Desta forma, não se considera como intrínseca ao processo de EESEP a obtenção de valores de parâmetros da rede. Entretanto, nem sempre tais valores são confiáveis, o que sugere uma sofisticação do processo de EESEP se lhe introduzindo parâmetros da rede como variáveis a serem estimadas. Neste sentido, recentemente, estimadores mais apurados foram propostos, em que variáveis de estado e parâmetros de elementos da rede integram o processo de estimação.

Suspeita-se da existência de erro, no valor de um determinado parâmetro, quando as medidas incidentes a ele apresentam resíduos normalizados elevados.

Conforme mencionado anteriormente, no capitulo 1, os parâmetros do sistema, disponíveis no banco de dados das empresas, podem estar incorretos, fruto de:

- Dados imprecisos fornecidos por fabricantes;

- Erros de modelagem que são comuns quando se considera, por exemplo, um modelo de parâmetros concentrados ( $\pi$-nominal), ao invés de um modelo de parâmetros distribuídos;

- Estimativas incorretas do comprimento das linhas de transmissão;

- Aproximações feitas nos modelos de transformadores; 
- A não consideração da dependência das condições climáticas de alguns parâmetros de linha (por exemplo, a resistência);

- Alterações de projeto não atualizadas na base de dados (por exemplo, seção de linha aérea que se torna subterrânea);

- Preenchimento incorreto da base de dados acessada pelo estimador;

- Erros que surgem durante a operação da rede elétrica. Tais erros ocorrem devido a problemas elétricos ou mecânicos, em dispositivos de monitoração de tapes de transformadores, ou em função de uma modificação local de um tap, sem a posterior informação ao Centro de Controle. Nessa categoria, pode citar-se ainda a colocação em operação de banco de capacitores, chaveamento de reatores de linha, entre outros;

- Discrepâncias nos valores dos parâmetros usados por diferentes setores de uma mesma empresa, o que traz um impacto direto sobre o desempenho do processo de EESEP (FREUND, 2006).

A título de ilustração, na Tabela 2.1 mostram-se os valores dos parâmetros de algumas linhas de transmissão, obtidos da base de dados pertencente aos setores de planejamento e operação da empresa LIGHT, bem como os valores unificados, em uma base de dados construída com a ajuda de especialistas. Note que alguns dados não apresentam conformidade alguma e se mostraram, posteriormente, inválidos (FREUND, 2006). 
Tabela 3.1 - Discrepâncias entre fontes de dados de parâmetros.

\begin{tabular}{|c|c|c|c|c|c|c|}
\hline \multirow{3}{*}{ Linha } & \multicolumn{6}{|c|}{ Base de Dados (Valores em \%) } \\
\hline & \multicolumn{2}{|c|}{ Operação } & \multicolumn{2}{|c|}{ Planejamento } & \multicolumn{2}{|c|}{ Unificada } \\
\hline & $\mathbf{r}$ & $\mathbf{x}$ & $\mathbf{r}$ & $\mathbf{x}$ & $\mathbf{r}$ & $\mathbf{x}$ \\
\hline Botafogo - Samaritano 1 & 0.06000 & 0.17000 & 0.07796 & 0.14152 & 0.06287 & 0.16880 \\
\hline Botafogo - Samaritano 2 & 0.06000 & 0.17000 & 0.07796 & 0.14152 & 0.06287 & 0.16880 \\
\hline Samaritano - Baependi & 0.04035 & 0.65283 & 0.04450 & 0.64175 & 0.02603 & 0.21360 \\
\hline Samaritano - F. Caneca & 0.03744 & 0.56520 & 0.07728 & 0.64152 & 0.04982 & 0.40880 \\
\hline Baependi - F. Caneca & 0.03523 & 0.51350 & 0.03511 & 0.51241 & 0.01612 & 0.27170 \\
\hline F. Caneca - Grajaú 1 & 0.34440 & 1.96200 & 0.34440 & 1.96200 & 0.34440 & 1.96200 \\
\hline F. Caneca - Grajaú 2 & 0.34440 & 1.96200 & 0.34440 & 1.96200 & 0.34440 & 1.96200 \\
\hline Grajaú - A. Campista & 0.02970 & 0.16920 & 0.02970 & 0.16920 & 0.02970 & 0.16920 \\
\hline A. Campista - F. Caneca & 0.32110 & 1.82970 & 0.32110 & 1.82970 & 0.32110 & 1.82970 \\
\hline A. Campista - A. Campista & 0.18020 & 0.33270 & 0.10400 & 0.34380 & 0.24866 & 0.34355 \\
\hline Grajaú - R. Comprido & 0.21540 & 1.25900 & 0.21540 & 1.25900 & 0.21540 & 1.25900 \\
\hline R. Comprido - F. Caneca & 0.12060 & 0.68700 & 0.12060 & 0.68700 & 0.12060 & 0.68700 \\
\hline R. Comprido - R. Comprido & 0.03270 & 0.13290 & 0.03270 & 0.13290 & 0.03482 & 0.12798 \\
\hline Grajaú - Leopoldo & 0.05800 & 0.23060 & 0.05800 & 0.23060 & 0.03500 & 0.19960 \\
\hline Leopoldo - F. Caneca & 0.31390 & 1.78800 & 0.31390 & 1.78800 & 0.31390 & 1.78800 \\
\hline Leopoldo - Leopoldo & 0.00420 & 0.01720 & 0.00420 & 0.01720 & 0.00455 & 0.01659 \\
\hline Grajaú - Mangueira & 0.06370 & 0.24520 & 0.06250 & 0.26520 & 0.03070 & 0.1752 \\
\hline Mangueira - Uruguay & 0.06860 & 0.39050 & 0.06860 & 0.39050 & 0.06860 & 0.39050 \\
\hline Uruguay - F. Caneca & 0.24960 & 1.42190 & 0.24960 & 1.42190 & 0.24960 & 1.42190 \\
\hline Mangueira - Mangueira & 0.18020 & 0.33270 & 0.10400 & 0.34380 & 0.24866 & 0.34355 \\
\hline Uruguay - Uruguay & 0.00420 & 0.01720 & 0.01170 & 0.38400 & 0.01045 & 0.03962 \\
\hline
\end{tabular}

A situação relatada na Tabela 3.1 é típica de um processo de implantação de um estimador de estado. Neste caso, dados errôneos, que não apresentam impactos claramente observados por estudos realizados em alguns setores da empresa, são imediatamente revelados quando utilizados no processo de EESEP. Tal fato reforça a necessidade de estratégias para a detecção e correção de EPs, tornando mais eficaz o processo de EESEP.

O uso de parâmetros inadequados pode fazer o sistema operar fora da margem de segurança. Por outro lado, o uso de parâmetros mais exatos pode aumentar a margem de transferência de potência, trazendo benefícios econômicos. Através de um estudo econômico, em (KUSIC; GARRISON, 2004) foi demostrado que erros nos parâmetros de linhas de transmissão podem acarretar prejuízos na ordem de milhões de dólares por ano (a análise acima referida foi realizada para companhias americanas).

Os erros nos parâmetros afetam: análise de contingências, análise de curto circuito, cálculo de estabilidade de máquinas, planejamento de transmissão, estimação de estado, etc.

Do ponto de vista do processo de EESEP, o erro em um parâmetro elétrico de um elemento da rede produz efeito similar ao de um conjunto de EGs correlacionados, envolvendo as medidas associadas a tal elemento. Assim, por exemplo, as medidas de fluxo 
no elemento e as de injeção em suas barras terminais poderão ser colocadas sob suspeita de estarem com EGs.

Embora em muitos trabalhos sobre estimação de parâmetros alguns autores mencionem brevemente a importância de sua inclusão no processo de EESEP, poucos realizam estudos que caracterizam tal necessidade com resultados numéricos (ZARCO; EXPOSITO, 2000).

Em (STUART, 1973) realizou-se uma análise de sensibilidade dos resíduos das medidas, para estudar os efeitos de certos EPs (capacitância, indutância, resistência das linhas e posição de tap de transformador), sobre o processo de EESEP. A partir de um único caso base, com baixo nível de redundância global, foi simulada a ocorrência de erros simultâneos nos parâmetros de três linhas distintas e, em seguida, analisaram-se os efeitos desses erros no processo de EESEP. O resultado mais importante dessa pesquisa foi a verificação de que a indutância da linha responde pela introdução dos maiores erros sobre os valores estimados.

Já em (ZARCO; EXPOSITO, 1996) é apresentado um estudo detalhado da influência dos EPs no processo de EESEP, através de simulações com diversos sistemas associados a planos de medição com baixos e elevados níveis de redundância. Concluiu-se o seguinte:

- A estimação de parâmetros pode ser realizada em pequenas subredes locais, em torno do parâmetro suspeito, evitando com isso processamento desnecessário. Essa conclusão é importante em razão de afirmar que medidas eletricamente distantes são inúteis à estimação de parâmetros suspeitos;

- Na consideração de subredes locais, em relação ao parâmetro a ser estimado, quanto maior a precisão das medidas locais, maior será a influência do parâmetro na estimação;

- A presença de medidas de fluxo de potência é crucial para uma correta identificação de EPs, bem como para uma correta estimação de parâmetros, principalmente no tocante a parâmetros série.

Em (MEZA et al., 2006a) focalizou-se o problema de detectar, identificar e corrigir EPs de elementos da rede elétrica, para que o processo de EESEP alcance resultados confiáveis. Testes foram realizados considerando-se diferentes cenários de carregamento, 
magnitudes e localizações do erro, sistemas de medição, etc. Para analisar a influência dos EPs sobre a EESEP, foi utilizado o sistema IEEE-14 barras e 20 linhas. Para contemplar diferentes situações de operação, foram considerados 288 cenários de carga, correspondentes a um ciclo diário discretizado em intervalos de 5 minutos. Para cada um desses cenários foram simuladas medições e executado um programa de estimação de estado.

Sintetizando as conclusões obtidas, através das simulações realizadas sobre influência de EPs no processo de EESEP, em (MEZA et al., 2006) destacou-se que:

- Constata-se a deterioração do processo de EESEP, causada por EPs, a partir dos resíduos normalizados das medidas, que crescem à medida que aumenta a magnitude do referido erro;

- Sistemas de medição de redundância elevada exercem influência positiva para evidenciar a presença de erros em parâmetros;

- O efeito de um erro de parâmetro é mais significativo na primeira vizinhança do ramo com erro de parâmetro;

- Medidas mais precisas permitem evidenciar melhor a presença de EPs;

- O nível de precisão das medidas deve ser tal que permita reduzir a incerteza que se possa ter sobre o valor do parâmetro suspeito;

- Erros nas resistências são menos influentes do que erros nas reatâncias em série de ramos da rede;

- Medidas de fluxo de potência auxiliam mais do que as de injeção, para o tratamento de erros em parâmetros.

Recentemente, Assumindo, também, a hipótese supracitada, em (STACCHINI DE SOUZA et al., 2009) foi desenvolvido um método off-line para detecção, identificação e correção de EPs série de linhas de transmissão. Após a identificação dos parâmetros suspeitos de estarem com erros, através da análise dos resíduos normalizados, o problema de estimação de parâmetros é tratado como um problema de otimização que é resolvido através de algoritmo genético. Formula-se uma função aptidão onde se busca diminuir o resíduo normalizado das medidas incidentes aos ramos suspeitos, ou seja, das medidas que possuem resíduos normalizados maiores que um valor pré-estabelecido. O processo de estimação de estado tradicional é utilizado de forma acessória, participando na avaliação da 
aptidão dos indivíduos gerados durante o processo de busca. O método pode ser utilizado em conjunto com qualquer estimador de estado, sem a exigência de mudança alguma nas rotinas presentes em tal aplicativo, nem mesmo de aumento da redundância de medidas para realizar a estimação de parâmetros. Resultados de simulações computacionais mostram que o método possibilita o tratamento de EPs simples e múltiplos (em ramos adjacentes e/ou não adjacentes).

Com base nos estudos relativos à influência dos EPs sobre o processo de EESEP, pode-se afirmar que é drástico o efeito de um erro de parâmetro, normalmente intolerável. Além disto, tais erros são menos evidentes que os EGs e topológicos, podendo não ser identificados, levando assim a erros permanentes nos dados fornecidos pelo estimador.

A próxima seção, seção 3.2, abrange diferentes metodologias para detecção e identificação de EPs. Em seguida, na seção 3.3, encontram-se metodologias para estimação de parâmetros.

\subsection{Detecção e Identificação de Erros de Parâmetros}

Conforme discutido inicialmente, a presença de erros nos parâmetros da rede pode degradar significativamente os resultados do processo de EESEP, fazendo com que telemedidas corretas sejam identificadas como portadoras de EGs e conseqüentemente se perca a confiança em tal processo. Para contornar este problema, torna-se necessário identificar ramos da rede com parâmetros suspeitos e estimar seus valores.

Um aspecto relevante no problema da estimação de parâmetros é a necessidade prévia de identificar os parâmetros suspeitos. Algumas vezes, a experiência do operador, ou certas informações fornecidas pela equipe de manutenção, podem permitir uma seleção manual dos parâmetros suspeitos. Porém, na maioria dos casos, exigir-se-á um algoritmo.

Do ponto de vista do estimador de estado, um erro de parâmetro tem o mesmo efeito que um conjunto de erros correlacionados atuando sobre todas as medidas adjacentes a linha com parâmetro errôneo, isto é, as medidas de fluxo sobre o ramo e as injeções em seus nós extremos. Isto pode ser entendido, reescrevendo a equação referente à modelagem das medidas, da seguinte forma (VAN CUTSEM; QUINTANA, 1988): 
$z_{s}=h_{s}\left(x^{t}, p^{t}\right)+w_{s}=h_{s}\left(x^{t}, p^{*}\right)+\left[h_{s}\left(x^{t}, p^{t}\right)-h_{s}\left(x^{t}, p^{*}\right)\right]+w_{s}$

Onde:

$z \quad$ é o vetor de medidas;

$h \quad$ é o vetor das funções não lineares relacionadas às medidas;

$w \quad$ é o vetor de erro das medidas;

$s \quad$ é o sub-índice que indica o conjunto de medidas adjacentes ao parâmetro errôneo;

$\mathrm{x}^{\mathrm{t}} \quad$ é o vetor de valores verdadeiros das variáveis de estado;

$\mathrm{p}^{\mathrm{t}} \quad$ é o vetor de valores verdadeiros dos parâmetros;

$\mathrm{p}^{*} \quad$ é o vetor de valores errôneos dos parâmetros.

O termo entre colchetes, na Equação (3.1), corresponde a um vetor de erro de parâmetro, que pode ser considerado como um vetor adicional de erro das medidas. Se o erro do parâmetro for considerável, poderá haver detecção de EGs por parte do estimador e as medidas adjacentes ao ramo com erro de parâmetros possuirão resíduos elevados (VAN CUTSEM; QUINTANA, 1988). Pode-se linearizar este vetor de erro adicional da seguinte maneira:

$$
\mathrm{h}_{\mathrm{s}}\left(\mathrm{x}^{\mathrm{t}}, \mathrm{p}^{\mathrm{t}}\right)-\mathrm{h}_{\mathrm{s}}\left(\mathrm{x}^{\mathrm{t}}, \mathrm{p}^{*}\right) \approx\left[\frac{\partial \mathrm{h}_{\mathrm{s}}}{\partial \mathrm{p}}\right] \mathrm{e}_{\mathrm{p}}
$$

sendo $e_{p}=p^{*}-p^{t}$ o erro do parâmetro.

Assim, aqueles elementos da rede, cujas medidas associadas apresentarem resíduos normalizados elevados, serão considerados como suspeitos, em termos dos parâmetros que os representam, sendo recomendada a estimação de seus parâmetros.

Em (LIU et al., 1992), supõe-se que os dados com erros não gaussianos, isto é, medidas com EGs, tenham sido identificadas e eliminadas previamente. Logo, a presença persistente de valores elevados em certos resíduos normalizados é uma indicação da presença de EPs. O método proposto em (LIU; LIM, 1995) baseia-se essencialmente na mesma idéia. 
Em (DO COUTTO FILHO et al., 2008), foi proposto um algoritmo para detecção e identificação de EPs, baseado na análise dos resíduos obtidos através de um processo de EESEP convencional. Este algoritmo realiza-se através dos seguintes passos:

i. Executar o processo de EESEP convencional, para o plano de medição atual;

ii. Se houver medidas com resíduos normalizados elevados (e.g., acima de um limite superior a 3), ir para o passo (III). Caso contrário, encerra-se o processo;

iii. Com base nos resíduos normalizados que violam o limite pré-estabelecido para suspeição, formar uma lista ordenada de medidas;

iv. A cada medida da lista ordenada, associam-se ramos da rede (uma medida está associada ou é adjacente a um determinado ramo, quando a equação que relaciona tal medida com as variáveis de estado depender do parâmetro deste ramo);

v. O ramo que reunir o maior número de medidas a ele associadas será o $1^{\circ}$ da lista de suspeitos, e assim sucessivamente.

\subsection{Classificação dos métodos para o tratamento de erros de parâmetros}

Nesta seção serão descritos alguns dos métodos existentes para estimação de parâmetros, sendo comentadas suas vantagens e desvantagens.

Vários pesquisadores têm estudado o problema de estimação de parâmetros. Em um dos primeiros artigos sobre o assunto (MERRILL; SCHWEPPE, 1973), foi proposta a estimação de parâmetros da rede através de uma única amostra de medidas. Uma dificuldade com esta abordagem é o fato de que raramente existem medidas suficientes, nas vizinhanças dos parâmetros suspeitos, para habilitar sua estimação através de uma única amostra de medidas. Em razão disto, uma abordagem alternativa tem sido proposta, em que se utilizam medidas de diversas amostras.

Foi desenvolvido, em (SCHWEPPE, 1973), um algoritmo para estimação de parâmetros que processa, simultaneamente, medidas de diversas amostras. 
Em (SLUTSKER; CLEMENTS, 1996), constatou-se que os algoritmos de processamento simultâneo de medidas, de diversas amostras, são indicados apenas para aplicação off-line.

De uma forma geral, os métodos desenvolvidos para estimação de parâmetros podem ser divididos em dois grupos (ABUR; EXPÓSITO, 2004):

Grupo 1: Métodos baseados na análise da sensibilidade dos resíduos;

Grupo 2: Métodos que aumentam o vetor de variáveis de estado.

Os métodos referentes a esses dois grupos dependem de uma estimação de estado convencional para a determinação dos ramos suspeitos de estarem com EPs, que são ramos incidentes às medidas com resíduos elevados.

\section{Grupo 1: Métodos baseados na análise da sensibilidade dos resíduos}

Os métodos deste grupo utilizam o vetor de variáveis de estado convencional (tensões complexas) e baseiam-se na análise da sensibilidade dos resíduos das medidas, incidentes aos ramos suspeitos.

Os métodos baseados na análise de sensibilidade residual são executados no final do processo de EESEP convencional e aproveitam os cálculos necessários à identificação dos parâmetros suspeitos. Logo, os métodos deste grupo são mais plausíveis de serem incorporados aos estimadores de estado convencionais, pois, os mesmos não exigem muitas alterações no código "computacional" desses estimadores. Deve-se destacar que muitas vezes é necessário realizar diversas iterações do laço seqüencial: estimação de estado estimação de parâmetro.

Em razão de esses métodos utilizarem o vetor de variáveis de estado convencionais, sem a inclusão dos parâmetros suspeitos, os mesmos permitem executar processo de estimação de parâmetros logo depois de finalizado o processo de estimação de estado.

Em (VAN CUTSEM; QUINTANA, 1988) propuseram um método para estimação de parâmetros que se baseia na análise do efeito dos EPs nos resíduos das medidas. Embora o método se aplique a qualquer parâmetro da rede, maior ênfase foi dada para erros na posição de tap de transformadores. 
O método proposto em (VAN CUTSEM; QUINTANA, 1988), baseia-se na sensibilidade entre resíduos e erros das medidas:

$$
r=\Gamma_{r} w
$$

Onde:

$r \quad$ é o vetor de resíduo das medidas

$w \quad$ é o vetor de erro das medidas;

Sendo $\Gamma_{r}$ a matriz de sensibilidade residual dada por:

$$
\Gamma_{r}=I-H G^{-1} H^{T} W
$$

Onde:

$G \quad$ é matriz ganho

$$
G=H^{T} W H
$$

W é a matriz de ponderação que normalmente é a inversa da matriz de covariância das medidas;

$H \quad$ é a matriz jacobiana linearizada, que relaciona as medidas com as variáveis de estado; e

I é a matriz identidade.

Assim, a partir das Equações (3.3) e (3.4), pode-se estabelecer uma relação linear entre os resíduos das medidas afetadas $r_{s}$ e o erro do parâmetro $e_{p}$, como é mostrado a seguir:

$$
r_{s}=\left(\left(\Gamma_{r}\right)_{s s} \frac{\partial h_{s}}{\partial p}\right) e_{p}+\bar{r}_{s}
$$

Onde: 
$\left(\Gamma_{r}\right)_{s s}$ é a sub-matriz de sensibilidade $(s x s)$ de $\Gamma_{r}$ correspondente às $s$ medidas adjacentes ao parâmetros suspeito; e $\bar{r}_{s}$ é o vetor de resíduo, que seria obtido na ausência de erros nos parâmetros.

A Equação (3.6) pode ser interpretada como um modelo linear, que relaciona os resíduos das "s" medidas, $r_{s}$, com o erro do parâmetro $e_{p}$, na presença de um ruído $\bar{r}_{s}$, sendo a determinação de $e_{p}$ um problema de estimação local, no qual os resíduos devem ser ponderados de acordo com a suas propriedades estatísticas (apresentadas na seção 2.3, capítulo 2).

Em (VAN CUTSEM; QUINTANA, 1988) demonstra-se que, a partir da Equação (3.6), a estimação "ótima" de $e_{p}$, a partir de $r_{s}$, pode ser dada por:

$$
\hat{e}_{p}=\left[\left(\frac{\partial h_{s}}{\partial p}\right)^{T} W_{s}\left(\Gamma_{r}\right)_{s s}\left(\frac{\partial h_{s}}{\partial p}\right)\right]^{-1}\left(\frac{\partial h_{s}}{\partial p}\right)^{T} W_{s} r_{s}
$$

No entanto, conforme demonstrado em (VAN CUTSEM; QUINTANA, 1988), a resolução deste sistema pode apresentar um esforço computacional elevado, tendo em vista que o mesmo envolve a obtenção da matriz $\left(\Gamma_{r}\right)$ ss. Para evitar tal problema, uma expressão equivalente foi proposta em (VAN CUTSEM; QUINTANA, 1988), a partir da expressão que se permite obter da Equação (3.4):

$$
W_{s}\left(\Gamma_{r}\right)_{s s}=W_{S}-W_{S} H_{S} G^{-1} H_{S}^{T} W_{S}
$$

Logo, define-se o vetor $\boldsymbol{\delta}_{s}$, de dimensão $n$ :

$$
\delta_{s}=H_{s}^{T} W_{s}\left(\frac{\partial h_{s}}{\partial p}\right)
$$

Cujos componentes são dados pela seguinte expressão:

$$
\delta_{s i}=\sum_{k=1}^{s} \frac{\partial h_{k}}{\partial x_{i}} \frac{\partial h_{k}}{\partial p} \frac{1}{\sigma_{k}^{2}}
$$


$\operatorname{com} i=1, \ldots, n$.

Usando as Equações (3.8) e (3.9), e sendo:

$$
H_{s p}=\frac{\partial h_{s}}{\partial p}
$$

a Equação (3.7) terá a seguinte forma:

$$
\begin{aligned}
& \hat{e}_{p}=\left[\left(H_{s p}^{T} W_{s} H_{s p}\right)-\left(H_{s p}^{T} W_{s} H_{s} G^{-1} H_{s}^{T} W_{s} H_{s p}\right)\right]^{-1}\left(H_{s p}^{T} W_{s} r_{s}\right) \\
& \hat{e}_{p}=\left[\left(H_{s p}^{T} W_{s} H_{s p}\right)-\left(\delta_{s}^{T} G^{-1} \delta_{s}\right)\right]^{-1}\left(H_{s p}^{T} W_{s} r_{s}\right)
\end{aligned}
$$

Logo, para um elemento de cujo parâmetro se suspeita, procede-se da seguinte maneira:

1. Identificar as medidas estimadas, adjacentes ao elemento sob suspeita (fluxos no elemento e injeções de potência nas barras terminais);

2. Se apenas uma medida for afetada, a equação (3.7) torna-se:

$$
\hat{e}_{p}=\left(\frac{\partial h_{s}}{\partial p}\right)^{-1} \frac{r_{s}}{\left(\Gamma_{r}\right)_{s s}}
$$

Ir para o passo 3 .

Caso contrário:

(a) Calcular $\frac{\partial h_{s}}{\partial p}, H_{s}$, substituir na Equação (3.9) e calcular $\delta_{s}$

(b) Obter $\hat{e}_{p}$ da Equação (3.12).

3. Corrigir o parâmetro $p^{\text {corrigido }}=p^{\text {anterior }}+\hat{e}_{p}$

4. Realizar uma nova estimação de estado e verificar se há parâmetros suspeitos.

Se existirem parâmetros suspeitos, voltar ao passo 2. Caso contrário, finalizar o algoritmo. 
Este método pode ser estendido para estimar simultaneamente vários erros nos parâmetros, sendo $p, e_{p}$, etc, vetores em lugar de escalares. Finalmente, a estimação de estado pode ser repetida utilizando os parâmetros atualizados, sendo o erro no parâmetro reestimado até que nenhuma melhora significativa seja alcançada.

Uma das primeiras pesquisas para identificação de EPs, através da análise estatística dos resíduos das medidas, foi proposta em (ABOYTES; CORY, 1975). Neste trabalho, desenvolveu-se um estudo estatístico dos efeitos dos EGs, topológicos e de parâmetros, no processo de estimação de estado, donde se obtiveram as seguintes conclusões: (i) o uso de medidas de injeções nodais aumenta a dificuldade de identificar erros de configuração e de parâmetros; (ii) os EPs podem ser identificados mais efetivamente quando o fluxo de potência de uma linha, cujo parâmetro está com erro, é medido somente em um dos terminais.

Baseando-se na forte relação entre o fluxo de potência reativa, através do transformador, e a posição do tap do mesmo, (FLETCHER; STADLIN, 1983) lançaram um método para estimação da posição de taps de transformadores. A diferença entre os fluxos de potência reativa, medidos e calculados, através do transformador, é utilizada para verificar se a posição do tap é correta ou não. Os autores definem a posição inicial do tap como sendo metade da escala de variação do transformador. Já em (SMITH, 1985), o valor inicial do tap é definido como sendo 1 p.u.

A métodologia proposta em (LIU; LUN, 1992) também se baseia na análise dos resíduos das medidas, e possibilita a detecção, identificação e correção de erros de parãmetros. As limitações deste método são as seguintes: (i) grande número de iterações, pois o algoritmo encerra duas estimações independentes, a primeira para estimar os resíduos $^{11}$ e a segunda para estimar os EPs; (ii) as matrizes utilizadas no processo de estimação dos EPs são geralmente singulares, o que provoca problemas de observabilidade; e (iii) os autores não consideram a possibilidade de EGs.

De uma forma geral, pode-se dizer que os métodos baseados na análise da sensibilidade dos resíduos possuem as seguintes vantagens: não degradam o nível de redundância e não exigem rotinas adicionais às já existentes para implementação do processo convencional de estimação de estado. Porém, para alcançar a mesma precisão dos

\footnotetext{
${ }^{11}$ Através de um estimador por mínimos quadrados.
} 
métodos que aumentam o vetor de estado, exigem a realização de diversos ciclos de estimação de estado e parâmetros (ABUR; EXPOSITO, 2004).

\section{Grupo 2: Métodos que aumentam o vetor de variáveis de estado}

Os métodos deste grupo aumentam o vetor de variáveis de estado, para incluírem os parâmetros suspeitos, como se estes fossem variáveis independentes. Desta maneira, os parâmetros são estimados juntamente com as tensões complexas das barras do sistema de potência, o que se denomina de estimação simultânea de estado e parâmetros. Note-se, entretanto, que a execução preliminar da estimação de estado convencional pode ser necessária, para identificar quais parâmetros deverão ser incluídos no vetor de variáveis de estado. Além disso, torna-se necessário alterar o código computacional do processo convencional de EESEP.

Uma vez que os parâmetros suspeitos estejam incluídos no vetor de variáveis de estado, a resolução do sistema de equações pode ser obtida de duas formas:

1. Equações normais;

2. Filtro de Kalman.

Assim, os métodos que utilizam o vetor de variáveis de estado aumentado, no processo de estimação, diferem principalmente no modo de solução, conforme apresentado a seguir.

\subsubsection{Métodos que utilizam Equações normais}

Uma das alternativas para trabalhar com este modelo aumentado realiza-se através das equações normais, o que corresponde exatamente à mesma formulação do estimador de estado convencional por mínimos quadrados ponderados, porém com o vetor de variáveis de estado aumentado. Assim, o problema consiste em encontrar um vetor de variáveis de estado aumentado, tal que torne mínima a seguinte função objetivo:

$$
J(x, p)=\sum_{i=1}^{m} w_{i}\left[z_{i}-h_{i}(x, p)\right]^{2}
$$


onde a dependência em $p$ afete somente o conjunto $s$ de medidas que dele dependam, sendo:

$J$ a função objetivo que depende do vetor de variáveis de estado $x$ e do parâmetro $p$;

$m \quad$ o número de medidas disponíveis.

Valores prévios, $p_{o}$, para os parâmetros a serem estimados, podem ser incluídos no modelo como medidas adicionais (informação a priori). Assim, supondo que, além das medidas $z_{i}$, exista a priori um valor aproximado para o parâmetro suspeito $p_{o}$, a função objetivo, estabelecida pela Equação (3.14), pode ser modificada, resultando em:

$$
J(x, p)=\sum_{i=1}^{m} w_{i}\left[z_{i}-h_{i}(x, p)\right]^{2}+w_{p}\left(p-p_{o}\right)^{2}
$$

onde $w_{p}$ é o peso arbitrário atribuído àquelas medidas adicionais.

Na maioria dos trabalhos existentes, na literatura a respeito do assunto, considera-se que a Equação (3.15), ao invés da Equação (3.14), poderia ser utilizada para assegurar a observabilidade de $p$. No entanto, como descrito na análise apresentada em (ZARCO; EXPOSITO, 2000), isto não é garantido.

Por um lado, se $p$ não é observável com as medidas existentes, a medida adicional

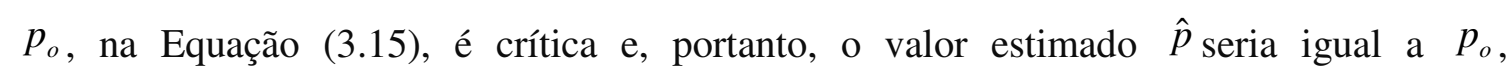
independente do valor atribuído a $w_{p}$. Por outro lado, se existir suficiente redundância para estimar os parâmetros, o valor atribuído a $w_{p}$ é crucial, já que pode influenciar significativamente no valor estimado $\hat{p}$. Desta forma, torna-se importante o valor da ponderação $w_{p}$, que é o inverso da variância da medida adicional $p_{o}$ (informação a priori), em relação às ponderações das tele-medidas $w_{i}$.

Atribuir a $w_{p}$ um valor muito pequeno em relação a $w_{i}$, equivale a rejeitar completamente a informação a priori $p_{o}$ e, portanto, o valor do parâmetro estimado $\hat{p}$ 
seria determinado exclusivamente pelas medidas disponíveis no sistema. Por outro lado, se for atribuído um valor muito elevado a $w_{p}$, em relação a $w_{i}$, um valor estimado $\hat{p}$ similar a $p_{o}$ será obtido, independente dos valores das medidas.

De forma a manter um nível de redundância local razoável, o número de parâmetros suspeitos ou variáveis extras, incluídas no vetor de variáveis de estado, deverá ser tão reduzido quanto possível. Por exemplo, sabe-se que muitas vezes os parâmetros por unidade de comprimento de uma linha da rede estão corretos, mas não se dispõe do valor correto sobre o comprimento total da linha. Para casos como esses, em (ABUR; EXPOSITO, 2004) estabelece-se o comprimento total da linha $(L)$ como parâmetro a ser estimado. Assim, para uma linha entre os nós $i$ e $j$, as seguintes expressões poderiam ser utilizadas na construção da matriz de admitâncias, usada no cálculo do vetor residual:

$$
\begin{aligned}
& \text { série }:\left(g_{i j}+j b_{i j}\right) / L \\
& \text { shunt }: j b_{i j}^{s h} L
\end{aligned}
$$

onde $g_{i j}, b_{i j} e^{b_{i j} h}$ são os valores das admitâncias, obtidos da base de dados do sistema.

Um dos primeiros métodos referentes à estimação de estado e parâmetros, baseado no vetor de estado aumentado via equações normais, visando inicialmente à estimação de todos os elementos da matriz de admitâncias, foi proposto em (ALLAN; LAUGHTON, 1974). Além de permitir estimar variáveis de estado e parâmetros, através da diferença entre os valores dos parâmetros fornecidos pelo banco de dados e os seus valores estimados, esse método permite identificar se os ramos do sistema estão fora de operação ou não.

Independente da técnica adotada, estes produzem um aumento no número de elementos da matriz Jacobiana, já que são introduzidas tantas colunas extras, quantas forem as novas variáveis (parâmetros suspeitos) adicionadas ao vetor de variáveis de estado, sendo os elementos destas novas colunas as derivadas parciais das medidas, com respeito às novas variáveis de estado. Ilustra-se, a seguir, a matriz Jacobiana $H_{x p}$ resultante da introdução de um único parâmetro como variável de estado: 


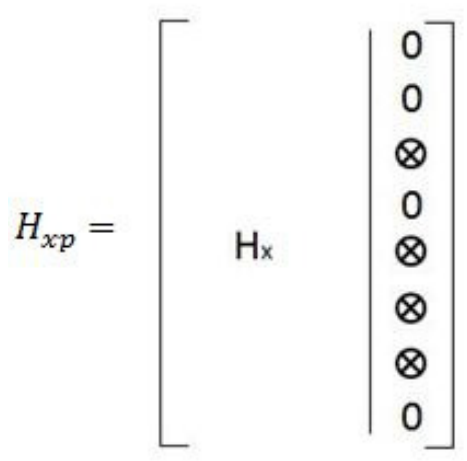

onde: $\mathrm{H}_{\mathrm{x}}$ corresponde a submatriz Jacobiana que relaciona as medidas com as variáveis de estado convencional; e $\otimes$ representa os elementos não nulos referentes às medidas adjacentes na coluna de derivadas, em relação ao parâmetro a ser estimado (derivadas das equações de medidas de fluxo e injeção, que dependem deste parâmetro).

No caso de existirem informações a priori, associadas às novas variáveis de estado, estas podem ser adicionadas ao vetor de medidas. Desta forma, tanto na matriz Jacobiana, como na matriz de covariância das medidas, aparecerão tantas linhas adicionais quantas forem as informações a priori adicionadas ao vetor de medidas, como ilustrado em (3.18), pela introdução da pseudomedida do parâmetro de um ramo.

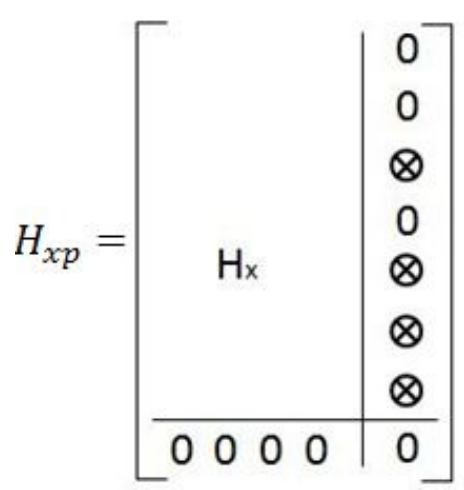

Por exemplo, supondo que o parâmetro $L$, da Equação (3.16), tenha sido adicionado ao vetor de variáveis de estado, os elementos de $H_{x p}$, correspondentes às medidas de potência (fluxos e injeções) no nó $i$, serão dados pelas Equações (3.19) e (3.20) (ABUR; EXPOSITO, 2004).

$$
\frac{\partial P_{i j}(L)}{\partial L}=\frac{\partial P_{i}(L)}{\partial L}=-\frac{P_{i j}}{L^{2}}
$$




$$
\frac{\partial Q_{i j}(L)}{\partial L}=\frac{\partial Q_{i}(L)}{\partial L}=-\left[Q_{i j}+V_{i}^{2} b_{i j}^{s h}\left(1+L^{2}\right)\right] / L^{2}
$$

onde $P_{i j}$ e $Q_{i j}$ referem-se aos fluxos de potência ativa e reativa, respectivamente, calculados para $L=1$. Expressões similares podem ser obtidas para as medidas de potência no nó $j$ (basta que se troque o subscrito).

Em (ALSAÇ et al., 1998) foi proposto um estimador generalizado que melhora o processo de obtenção de informação; sua principal contribuição é a inclusão de erros de topologia e EPs na estimação e no processo de análise de erros. Este estimador permite a modelagem das subestações suspeitas, ao nível de seção de barra. Para isto, o vetor de variáveis de estado convencional, formado pelas magnitudes e ângulos das tensões, ganha novas variáveis de estado, que são os fluxos de potência ativa e reativa, através dos disjuntores e dos ramos cujos parâmetros serão estimados. Novas pseudomedidas são também introduzidas ao modelo, permitindo assim uma melhor discriminação entre EGs, topológicos e EPs.

A metodologia proposta em (LIU; LUN, 1995) aumenta o vetor de variáveis de estado com os incrementos dos fluxos de potência, em lugar dos parâmetros errôneos. Portanto, a estimação a que se procede é para aqueles incrementos, sendo posteriormente calculados os parâmetros errôneos. Essa metodologia utiliza apenas uma amostra de medidas e então sofre os mesmos problemas de observabilidade, como se menciona no trabalho de (MERRILL; SCHWEPPE, 1973).

De uma forma geral, é possível afirmar que a limitação dos métodos que usam equações normais está relacionada a problemas de observabilidade, já que raramente o número de medidas disponível é suficiente para estimar todas as variáveis de estado aumentadas, uma vez que as mesmas aumentam, mas o conjunto de medidas continua o mesmo (ZARCO; EXPOSITO, 2000).

Para a solução deste problema, alguns métodos, os quais permitem executar a estimação de parâmetros que permanecem constantes por um determinado intervalo de tempo, em modo off-line, processam simultaneamente várias amostras de medidas, obtidas em diferentes instantes de tempo, com o objetivo de incrementar a redundância ao redor 
dos parâmetros suspeitos (REIG; ALVAREZ, 1989; ZARCO; EXPOSITO, 1996). Assim, no caso de realizar-se uma única vez a estimação de estado e supondo que exista um único parâmetro suspeito, a redundância será dada pela seguinte expressão:

$$
K_{1}=\frac{m}{n+1}
$$

sendo $m$ o número de medidas e $n$ o número de variáveis de estado convencional.

No caso de um aumento do número de parâmetros suspeitos $(n p)$ e desejando-se que a redundância continue sendo $K_{1}$, bastará que o número de amostras a serem processadas simultaneamente seja q. Desta forma, a redundância é dada por:

$$
K_{q}=\frac{m \times q}{n \times q+n p}
$$

sendo $q=n p$ para que $K_{1}=K_{q}$

Nestes métodos, da mesma maneira que para outros métodos de estimação existentes, se o sistema de medição apresenta níveis críticos de redundância, a estimação do parâmetro pode tornar-se muito difícil ou inviável.

Se forem utilizadas $q$ amostras simultâneas, para realizar o processo de estimação, o vetor de variáveis de estado, aumentado com os parâmetros suspeitos, será dado por:

$$
x=\left[x_{1}, x_{2}, \ldots, x_{q} \mid p\right]^{T}
$$

e o vetor de medidas por:

$$
z=\left[z_{1}, z_{2}, \ldots, z_{q}\right]^{T}
$$

onde $x_{i}$ e $z_{i}$ são, respectivamente, os vetores de variáveis de estado convencional e de medidas da amostra $i$ e $p$ é o vetor de parâmetros. 
No método descrito em (ZARCO;EXPOSITO, 1997) os vetores de variáveis de estado e de medidas são representados pelas Equações (3.23) e (3.24). A estrutura da matriz Jacobiana é dada por:

$$
\left[\begin{array}{ccccc}
H_{1} & & & & H_{1 p} \\
& H_{2} & & & H_{2 p} \\
& & \ddots & & \vdots \\
& & & H_{q} & H_{q p}
\end{array}\right]
$$

onde a nova coluna corresponde ao parâmetro suspeito. Nota-se que nenhuma informação prévia sobre o parâmetro é considerada.

Assim, substituindo as Equações (3.23) e (3.24), nas equações normais do processo de estimação de estado, o processo de convergência passa a ser dado pela seguinte expressão:

$$
G \Delta x=H^{T} W \Delta z
$$

onde a matriz ganho $G$ é dada por:

$$
G=H^{T} W H=\left[\begin{array}{ccccc}
G_{11} & & & & G_{1 p} \\
& G_{22} & & & G_{2 p} \\
& & \ddots & & \vdots \\
& & & G_{q q} & G_{q p} \\
G_{1 p}^{t} & G_{2 p}^{t} & \ldots & G_{q p}^{t} & G_{p p}
\end{array}\right]
$$

sendo:

$$
\begin{array}{ll}
G_{i i}=H_{i}^{T} W_{i} H_{i} & (i=1,2, \cdots, q) \\
g_{i p}=H_{i}^{T} W_{i} h_{i p} & (i=1,2, \cdots, q) \\
G_{P P}=\sum_{i=1}^{q} h_{i p}^{t} W_{i} h_{i p} &
\end{array}
$$


O vetor de termos independentes é dado por:

$$
H^{T} W \Delta Z=\left[b_{1}, b_{2}, \cdots, b_{q} \mid b_{p}\right]^{T}
$$

sendo:

$$
\begin{gathered}
b_{i}=H_{i}^{T} W_{i} \Delta z_{i} \quad(i=1,2, \cdots, q) \\
b_{p}=\sum_{p}^{q} h_{i p}^{t} W_{i} \Delta z_{i}
\end{gathered}
$$

Durante a aplicação deste método, é necessário construir e fatorar a matriz $G$, de forma a obter-se a correção do vetor de variáveis de estado $\Delta x$.Para redes de grande porte isto pode ser impraticável, mesmo utilizando técnicas de esparsidade. Entretanto, como geralmente o número de parâmetros a serem estimados é relativamente pequeno, podem utilizar-se métodos alternativos, cujo custo computacional é similar ao necessário para resolver, de forma seqüencial, vários problemas de estimação de estado independentes, como o descrito em (ZARCO;EXPOSITO, 1997). passos:

O método proposto em (ZARCO;EXPOSITO, 1997) resume-se nos seguintes

1. Triangularização e eliminação. Para cada bloco diagonal da matriz ganho $(i=1,2, \cdots, q)$.

(a) Calcular:

$$
\begin{aligned}
& \hat{h}_{i p}=W_{i} h_{i p} \\
& \Delta \hat{z}_{i}=W_{i} \Delta z_{i} \\
& g_{i p}=H_{i}^{T} \hat{h}_{i p} \\
& b_{i}=H_{i}^{T} \Delta \hat{z}_{i}
\end{aligned}
$$

(b) Calcular $G_{i i}$ e obter $y_{i}, b_{i}^{\prime}$ através de: 


$$
\begin{aligned}
& G_{i i}=H_{i}^{T} W_{i} H_{i} \\
& G_{i i} y_{i}=g_{i p} \\
& G_{i i} b_{i}^{\prime}=b_{i}
\end{aligned}
$$

(c) Atualizar $G_{p p}$ e $b_{p}$ :

$$
\begin{aligned}
& G_{p p}=G_{p p}+h_{i p}^{t} \hat{h}_{i p}-g_{i p}^{t} y_{i} \\
& b_{p}=b_{p}+h_{i p}^{t} \Delta \hat{z}_{i}-g_{i p}^{t} b_{i}^{\prime}
\end{aligned}
$$

2. Obter $\Delta p$ resolvendo:

$$
G_{p p} \Delta p=b_{p}
$$

3. Obter $\Delta x_{i}$ :

$$
\Delta x_{i}=b_{i}^{\prime}-y_{i} \Delta p
$$

Desta forma, uma vez obtido $\Delta p$, permite-se atualizar todas as variáveis de estado independentemente.

No que diz respeito ao método em pauta, cabe ressaltar que:

- Cada coluna de $h_{i p}$ é um vetor muito esparso, já que somente as medidas adjacentes têm derivadas com relação a $p$, diferentes de zero;

- Cada bloco diagonal $G_{i i}$ é utilizado somente uma vez no passo 1(b), porquanto somente a matriz ganho, correspondente a uma única variável de estado, deve ser armazenada;

- Se apenas um único parâmetro for estimado, $h_{i p}$ será um vetor coluna e $G_{p p}$ um escalar. 
No caso de se ter um pequeno número de parâmetros a estimar, o custo computacional será menor, em comparação ao custo do processamento seqüencial de vários problemas de estimação de estado independentes (ZARCO;EXPOSITO, 1997).

Para enfrentar o problema de um custo "computacional" elevado, decorrente da aplicação dos métodos que processam simultaneamente uma seqüência de amostras de medidas, a seguir serão apresentados os métodos baseados no filtro de Kalman, que utilizam um algoritmo recursivo, no qual estimativas a priori das variáveis de estado aumentadas são adicionadas ao vetor de medidas corrente, de modo a atualizar os parâmetros estimados.

\subsubsection{Métodos que utilizam o Filtro de Kalman}

A possibilidade de estimar o vetor de variáveis de estado aumentado, através de um algoritmo recursivo, baseado no filtro de Kalman, foi investigada inicialmente por (DEBS, 1974). Nesse trabalho, os parâmetros são modelados como sendo constantes e as tensões complexas das barras são atualizadas segundo o processo de Markov. O uso de modelos dinâmicos permitiu o emprego da estimação recursiva, na qual a informação a priori sobre as variáveis de estado e os parâmetros estimados é combinada com uma informação atual (medidas) para atualizar a estimação de parâmetros. As limitações de tal formulação são as seguintes: - problemas de convergência, quando aplicada a sistemas grandes e/ou com muitos parâmetros incorretos; - considera os parâmetros como constantes, limitando a flexibilidade do algoritmo, visto que alguns parâmetros, em razão das perdas por efeito corona, são variáveis com o tempo.

Para cada amostra em um instante de tempo $k$, a relação existente entre as medidas e as variáveis de estado aumentadas pode ser descrita por:

$$
z(k)=h(x(k), k, p)+w(k)
$$

onde a dependência explícita de $h$ em $k$ reflete a possibilidade de mudanças na rede de um instante para outro. Contudo, consideram-se os parâmetros constantes durante todo o período de análise. 
Para a estimação das variáveis de estado $\hat{x}(k)$, e $\hat{p}$ do sistema, deve-se tornar mínima a função objetivo dada por:

$$
J_{k}=\sum_{i=1}^{m}\left[z_{i}(k)-h_{i}(x(k), k, p)\right]^{T} W\left[z_{i}(k)-h_{i}(x(k), k, p)\right]
$$

No caso de se dispor de uma estimativa a priori do vetor de parâmetros $p_{o}$, esta pode ser incorporada ao problema como uma pseudo-medida; a idéia é obter melhor estimativa de $p$, em cada nova amostra de:

$$
p_{k-1}=p_{k}+e_{p}(k)
$$

onde o vetor de erro $e_{p}$ é um vetor aleatório gaussiano, com média zero e matriz de covariância $R_{p}(k)$.

No caso do modelo estático $W_{P}=R_{P}^{-1}=0$, o que permite o uso de um algoritmo de filtro de Kalman estendido, onde, numa amostra de tempo $k$, o seguinte algoritmo iterativo é usado:

$$
\left[\begin{array}{c}
\hat{x}_{k}^{i+1}(k) \\
\hat{p}_{k}^{i+1}(k)
\end{array}\right]=\left[\begin{array}{c}
\hat{x}_{k}^{i}(k) \\
\hat{p}_{k}^{i}(k)
\end{array}\right]+\Lambda^{i}(k)\left[\begin{array}{l}
A_{1} \\
A_{2}
\end{array}\right]
$$

em que:

$$
\begin{aligned}
& A_{1}=H_{x}^{T}(k) W\left[z(k)-h\left(\hat{x}_{k}^{i}(k), k, \hat{p}_{k}^{i}(k)\right)\right] \\
& \left.A_{2}=H_{x}^{T}(k) W\left[z(k)-h\left(\hat{x}_{k}^{i}(k)\right], k, \hat{p}_{k}^{i}(k)\right)\right]+W_{p, k-1}\left[\hat{p}_{k-1}^{i}(k-1)-\hat{p}_{k}^{i}(k)\right] \\
& H_{x}(k)=\left.\frac{\partial h}{\partial x}\right|_{\hat{x}_{k}^{i}(k), \hat{p}_{k}^{i}(k)}
\end{aligned}
$$




$$
\begin{aligned}
& H_{p}(k)=\left.\frac{\partial h}{\partial p}\right|_{\hat{x}_{k}^{i}(k), \hat{p}_{k}^{t}(k)} \\
& \Lambda^{i}(k)=\left[\begin{array}{cc}
H_{x}^{T}(k) W H_{x}(k) & H_{x}^{T}(k) W H_{p}(k) \\
H_{p}^{T}(k) W H_{x}(k) & W_{p, k=1}+H_{p}^{T}(k) W H_{p}(k)
\end{array}\right]^{-1}=\left[\begin{array}{cc}
\Lambda_{x x}^{i}(k) & \Lambda_{x p}^{i}(k) \\
\Lambda_{p x}^{i}(k) & \Lambda_{p p}^{i}(k)
\end{array}\right] \\
& R_{p, k}=\Lambda_{p p}(k)
\end{aligned}
$$

onde $\hat{p}$ é o vetor de parâmetros estimados e $i$ representa a iteração corrente $(i=1,2, \cdots)$.

Este processo iterativo (baseado no Filtro de Kalman) é de natureza recursiva, uma vez que a amostra de tempo $k$ é formada pelo vetor $z(k)$ e pelos parâmetros estimados no tempo $k-1$, com suas respectivas covariâncias previamente atualizadas.

Diferentemente do caso anterior, os parâmetros não são considerados constantes em (SLUTKER, 1995; SLUTSKER; CLEMENTS, 1996), pois, assim como as tensões e os ângulos das barras do sistema, tais parâmetros são também atualizados segundo o processo de Markov. Deste modo, permite-se a estimação de parâmetros variáveis com o tempo. Este método pode ser aplicado a pequenas subredes observáveis, as quais contenham os parâmetros suspeitos. Como conseqüência tem-se que:

- A solução é mais rápida, já que são resolvidos vários pequenos problemas, em vez de um único e maior;

- É possível a expansão adaptativa das áreas analisadas, de forma que sejam estimados inicialmente os parâmetros das linhas associadas a uma maior redundância de medidas. Em seguida, passa-se à estimação dos parâmetros de linhas, associadas a uma menor redundância.

O método baseia-se na operação de tornar mínima a função objetivo $\Phi$, descrita a seguir:

$$
\begin{aligned}
& \Phi\left[y\left(t_{i}\right)\right]=\frac{1}{2}\left[z\left(t_{i}\right)-h\left(x\left(t_{i}\right), p\left(t_{i}\right)\right)\right]^{T} W\left[z\left(t_{i}\right)-h\left(x\left(t_{i}\right), p\left(t_{i}\right)\right)\right] \\
& +\frac{1}{2}\left[y\left(t_{i}\right)-\bar{y}_{i}\left(t_{i}\right)\right]^{T} W_{x p}\left[y\left(t_{i}\right)-\bar{y}_{i}\left(t_{i}\right)\right]
\end{aligned}
$$


onde:

$$
y^{T}\left(t_{i}\right)=\left\lfloor x^{T}\left(t_{i}\right), p^{T}\left(t_{i}\right)\right\rfloor
$$

A condição necessária, para localizar o mínimo da função, é dada pela seguinte expressão:

$$
\frac{\partial \Phi\left[y\left(t_{i}\right)\right]}{\partial y\left(t_{i}\right)}=-H\left[y\left(t_{i}\right)\right]^{T} W\left[z\left(t_{i}\right)-h\left(y\left(t_{i}\right)\right)\right]+W_{x p}\left(t_{i}\right)\left[y\left(t_{i}\right)-\bar{y}\left(t_{i}\right)\right]=0
$$

onde:

$$
H\left[y\left(t_{i}\right)\right]=\frac{\partial h\left[y\left(t_{i}\right)\right]}{\partial y\left(t_{i}\right)}
$$

A matriz $W_{x p}\left(t_{i}\right)$ é a inversa da matriz de covariância de $y\left(t_{i}\right)$ :

$$
W_{x p}\left(t_{i}\right)=\left[\begin{array}{cc}
W_{x}\left(t_{i}\right) & 0 \\
0 & W_{p}\left(t_{i}\right)
\end{array}\right]
$$

Para a solução deste sistema não linear, utiliza-se o método de Newton-Raphson:

$$
\begin{gathered}
C \Delta y\left(t_{i}\right)=H\left[y\left(t_{i}\right)^{k}\right]^{T} W\left[z\left(t_{i}\right)-h\left(y\left(t_{i}\right)^{k}\right]-W_{x p}\left(t_{i}\right)\left[y\left(t_{i}\right)^{k}-\bar{y}\left(t_{i}\right)\right]\right. \\
y\left(t_{i}\right)^{k+1}=y\left(t_{i}\right)^{k}+\Delta y\left(t_{i}\right)
\end{gathered}
$$

onde:

$$
C=H\left[y\left(t_{i}\right)^{k}\right]^{T} W H\left[y\left(t_{i}\right)^{k}\right]+W_{x p}\left(t_{i}\right)-\left.\sum_{j=1}^{m_{p}}\left[\frac{z_{j}\left(t_{i}\right)-h_{j} y\left(t_{i}\right)}{R_{j j}}\right] \cdot \frac{\partial^{2} h_{j}\left[y\left(t_{i}\right)\right]}{\partial y\left(t_{i}\right)^{2}}\right|_{y\left(t_{i}\right)=y\left(t_{i}\right)^{k}}
$$

sendo $j$ o índice dos parâmetros e $m_{p}$ o número de parâmetros.

Para efetuar a estimação dos parâmetros, divide-se a rede em pequenas subredes, ou subsistemas locais, e, a cada iteração, são resolvidas as Equações (3.53)-(3.54), mediante a fatoração triangular da matriz $C$. Cada sub-rede é uma pequena porção da rede completa, 
composta de umas poucas linhas, com número suficiente de medidas para assegurar a observabilidade.

O método em questão é desenvolvido como um estimador de parâmetros adaptativo, que começa com poucas linhas altamente redundantes. Após a estimação das impedâncias destas linhas, as mesmas são utilizadas para ampliar a estimação de parâmetros para linhas que apresentem redundâncias menores. A última das soluções conterá todas as linhas da rede com adequada redundância, excluindo aquelas nas quais não se tornar possível realizar a estimação de parâmetros.

De acordo com (ABUR; EXPÓSITO, 2004), a necessidade de atualização da matriz de covariância dos EPs aumenta muito o custo computacional dos métodos baseados no Filtro de Kalman, principalmente quando é grande a quantidade de parâmetros suspeitos.

\subsubsection{Soluções alternativas para tratamento de erros de parâmetros}

Em (ZHU; ABUR, 2006) desenvolveu-se uma metodologia para identificação e correção de EPs que possibilita a discriminação entre EGs e EPs, mesmo quando estes ocorrem simultaneamente. A metodologia baseia-se na análise dos multiplicadores de Lagrange associados às restrições impostas sobre a ausência de EPs (restrições nulas). Isto é, inicialmente admiti-se a hipótese de que não existam EPs. Através de um processo de estimação de estado convencional, obtêm-se os resíduos das medidas que, por sua vez, são utilizados para obtenção dos multiplicadores de Lagrange. Os parâmetros suspeitos de estarem com erros são aqueles associados aos multiplicadores de Lagrange maiores, em módulo, que um limiar pré-estabelecido. Segundo os autores, a metodologia pode apresentar problemas quando dois ou mais EPs afetam o mesmo subconjunto de medidas.

Em (DO COUTTO FILHO et al., 2008) foi proposto uma metodologia off-line para depuração de erros em parâmetros série de linhas de transmissão. A idéia é criar um processo de estimação de estado imune/menos sensível aos EPs. Para isso foi explorado o conceito de ramo irrelevante ${ }^{12} /$ quase irrelevante. Estratégias suplementares, baseadas na mudança da ponderação das medidas, foram desenvolvidas para lidar com situações especiais em que a criação de ramos irrelevantes/quase irrelevantes tornava o sistema não

\footnotetext{
${ }^{12}$ Considera-se um ramo irrelevante para estimação de estado, quando nenhuma medida (fluxo ou injeção) estiver a ele associada, significando que tal ramo não participa do processo de estimação.
} 
observável. Foram apresentadas diversas simulações com o sistema de 14 barras do IEEE. Vale destacar que para possibilitar a identificação de EPs essa metodologia assume a hipótese de obtenção de amostras de medidas imunes de erros topológicos e de erros groseiros.

Assumindo, também, a hipótese supracitada, em (STACCHINI DE SOUZA et al, 2009) foi desenvolvido um método off-line para detecção, identificação e correção de EPs série de linhas de transmissão. Após a identificação dos parâmetros suspeitos de estarem com erros, através da análise dos resíduos normalizados, o problema de estimação de parâmetros é tratado como um problema de otimização que é resolvido através de algoritmo genético. Formula-se uma função aptidão onde se busca diminuir o resíduo normalizado das medidas incidentes aos ramos suspeitos, ou seja, das medidas que possuem resíduos normalizados maiores que um valor pré-estabelecido. O processo de estimação de estado tradicional é utilizado de forma acessória, participando na avaliação da aptidão dos indivíduos gerados durante o processo de busca. O método pode ser utilizado em conjunto com qualquer estimador de estado, sem a exigência de mudança alguma nas rotinas presentes em tal aplicativo, nem mesmo de aumento da redundância de medidas para realizar a estimação de parâmetros. Resultados de simulações computacionais mostram que o método possibilita o tratamento de EPs simples e múltiplos (em ramos adjacentes e/ou não adjacentes).

Vale destacar ainda que pesquisas recentes têm investigado a possibilidade de melhorar o processo de validação de parâmetros através da utilização de medidas fasoriais sincronizadas. Em (ZHU; ABUR, 2009), foi mostrado que em função da topologia de um SEP, para ser possível a identificação de EPs é necessário, em algumas situações, fazer uso de medidas fasoriais sincronizadas. No artigo de (ZHU; ABUR, 2010), foi proposto um algoritmo para detecção e identificação de EPs usando medidas fasoriais sincronizadas.

\subsection{Comparação entre as Metodologias de Tratamento de Erros de Parâmetros}

Os artigos publicados para tratamento de EPs não estabelecerem comparações precisas entre as diferentes metodologias. Com base em (ZARCO; EXPOSITO, 2000) e nas publicações mais relevantes, algumas conclusões podem ser estabelecidas: 
- No tocante à estimação do valor do parâmetro, a metodologia de aumento do vetor de variáveis de estado é preferida, em relação àquela que empregada análise de sensibilidade de resíduos. A série de linearizações realizadas no método de análise de sensibilidade de resíduos necessita de um número maior de iterações para obter precisão semelhante aquela do método do vetor aumentado;

- Embora o método do vetor aumentado por equações normais seja eficaz na estimação do valor do parâmetro, o mesmo não possui a característica de identificar os parâmetros suspeitos. A carência dessa fase de pré-identificação compromete a metodologia, tendo em vista poder iniciar a fase de estimação contemplando valores errados para os parâmetros. Além disso, ao adicionar-se um parâmetro ao vetor de variáveis de estado, reduz-se a eficiência computacional, devido ao aumento da dimensão da matriz ganho. Há ainda como conseqüência a redução no nível de redundância do sistema de medição. Mas esta limitação se contorna considerando várias amostras de medidas;

- A técnica de análise de sensibilidade de resíduos é eficaz na determinação de parâmetros suspeitos, apresentando, porém, baixa eficiência, na existência de mais de um erro de parâmetro;

- Em razão de não exigirem muitas alterações no código computacional dos estimadores de estado convencionais, os métodos para estimação de parâmetros baseados na análise de sensibilidade residual são mais plausíveis de se incorporarem àqueles estimadores, relativamente aos métodos que fazem uso de um vetor de estado aumentado;

- A utilização da teoria de filtro de Kalman é eficaz, pois melhora, recursivamente o valor do parâmetro, porém a necessidade de atualizar as matrizes de covariância torna essa metodologia computacionalmente ineficiente, principalmente quando o número de parâmetros a ser estimado é elevado;

- Um ponto em que não há controvérsia no tratamento de EPs diz respeito à natureza do processamento para estimação do parâmetro. Para parâmetros que permanecem essencialmente constantes, como a reatância das linhas, o melhor é realizar um processo off-line de estimação de parâmetros. Já no que se refere a variáveis dinâmicas, como taps de transformadores, o processo on-line é o mais indicado; 
- Vários trabalhos têm mostrado que é significativa a influência de erros nos parâmetros shunt das linhas de transmissão no processo de EESEP (MINGUEZ; CONEJO, 2007; LEFEBVRE et al., 2004; LEFEBVRE et al., 2006), principalmente em sistemas de transmissão com grande circulação (absorção e compensação) de potência reativa. Este é o caso, por exemplo, do sistema de transmissão da HydroQuébec Trans-Energie. Desta forma, é necessário o desenvolvimento de metodologias que possibilitem a depuração de parâmetros série e shunt de linhas de transmissão. 


\section{Capítulo 4}

\section{Metodologia Proposta para Depuração de Parâmetros Série e Shunt de Linhas de Transmissão}

\subsection{Introdução}

Propõe-se, neste capítulo, uma metodologia eficiente e prática para depuração (detectar, identificar e corrigir EPs) off-line de parâmetros série e shunt de linhas de transmissão disponíveis nos bancos de dados das companhias de energia elétrica.

A metodologia proposta faz uso de medidas de diversas amostras e compreende três fases que possibilitam: identificação de parâmetros suspeitos de estarem com erro (Fase 1); estimação dos parâmetros suspeitos (Fase 2); e validação dos valores estimados (Fase 3). Cada uma dessas Fases será apresentada nas próximas seções deste capítulo.

Conforme será apresentado no capítulo 5, resultados de diversas simulações, com os sistemas de 14, 30 e 57 barras do IEEE, têm demonstrado a alta precisão e confiabilidade da metodologia proposta, mesmo na ocorrência de erros múltiplos (em mais de um parâmetro) em ramos adjacentes.

Importa destacar ainda que a metodologia proposta foi utilizada com sucesso para depuração dos parâmetros de dois subsistemas da Hydro-Québec TransÉnergie, conforme será apresentado no capítulo 6. 


\subsection{Parâmetros a serem estimados}

Em estimação de estado utiliza-se, para representação de linhas de transmissão, o modelo $\Pi$ equivalente, apresentado na Figura 4.1. Tal modelo possibilita a representação de uma linha de transmissão, através de parâmetros concentrados.

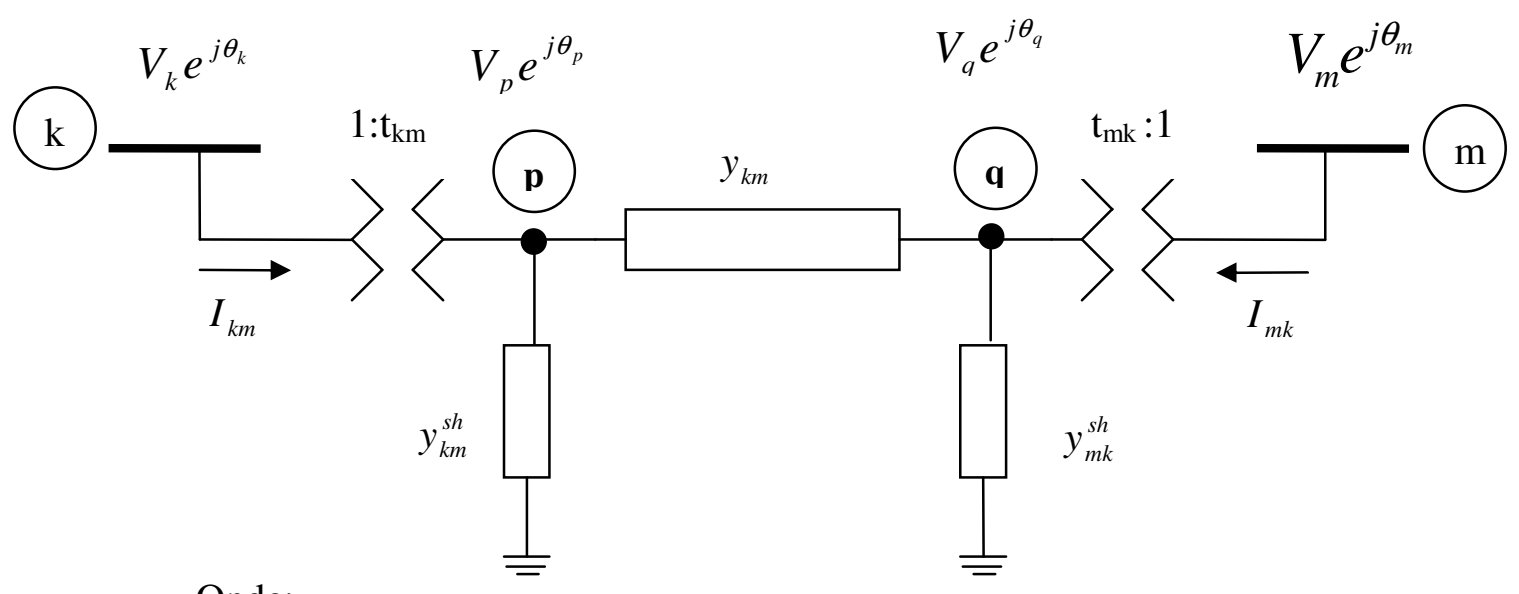

Onde:

$V_{k}, V_{p}, V_{m}$ - Magnitude de tensão das barras k, p e m.

$\theta_{k}, \theta_{p}, \theta_{m}-$ Ângulo de tensão das barras k,p e m.

$y_{k m}=g_{k m}+j b_{k m}-$ Admitância série da linha de transmissão k-m.

$y_{k m}^{s h}$ - Susceptância shunt da linha de transmissão k-m.

$1: t_{k m}=a_{k m} e^{j \theta_{p}}-$ Relação de transformação

Figura 4.1 Modelo $\prod$ - Equivalente generalizado

Importa destacar que, em termos de estimação de estado, como as medidas são tomadas apenas nas extremidades da linha, não há diferença entre a representação com parâmetros concentrados ou distribuídos, mesmo para linhas de transmissão aéreas classificadas como longas (STEVENSON, 1986).

Os parâmetros a serem estimados, pela metodologia proposta, são as condutâncias série $\left(G_{k m}\right)$, as susceptâncias série $\left(B_{k m}\right)$ e as susceptâncias shunt $\left(B_{k m}^{s h}\right)$ de linhas de transmissão aéreas, classificadas como médias e longas, isto é, com comprimento acima de $80 \mathrm{~km}$ (50 milhas) ${ }^{13}$.

\footnotetext{
${ }^{13}$ De acordo com (KUSIC; GARRISON, 2004) esses parâmetros são os principais responsáveis pelas perdas em um sistema elétrico de potência.
} 
Através dos parâmetros do modelo $\Pi$ equivalente, distingue-se uma linha aérea classificada como curta, das classificadas como médias e longas, através da sua susceptância shunt que será igual a zero. Isto porque uma linha aérea classificada como curta, isto é, com comprimento de até $80 \mathrm{~km}$ (50 milhas), possui uma capacitância em derivação muito pequena, que pode ser inteiramente desprezada, sem perda apreciável de precisão, sendo suficiente considerar apenas a resistência em série e a indutância em série, para todo o comprimento da linha.

\subsection{Metodologia Proposta}

A metodologia proposta compreende três fases que, num processo iterativo off-line, possibilitam a depuração de parâmetros de linhas de transmissão. As três fases são as seguintes:

Fase 1: Detecção e Identificação de ramos suspeitos de estarem com EPs;

Fase 2: Estimação dos parâmetros suspeitos;

Fase 3: Validação das estimativas dos parâmetros suspeitos.

\subsubsection{Fase 1: Detecção e Identificação de ramos suspeitos de estarem com erros de parâmetros}

O procedimento proposto para detecção e identificação de parâmetros suspeitos de estarem com erro faz uso dos resíduos normalizados das medidas. Tendo em vista que o procedimento proposto é off-line, é possível selecionar amostras de medidas isentas de EGs e topológicos. Desta forma, medidas com resíduos normalizados elevados são um indicativo da presença de EPs. Isto porque, para o processo de EESEP convencional, o erro no parâmetro de uma linha de transmissão causará um aumento nos resíduos normalizados das medidas analógicas incidentes àquela linha, isto é, medidas de fluxo de potência naquela linha ou de injeção de potência em uma das barras terminais daquela linha.

Em alguns trabalhos o processo de identificação de ramos suspeitos de estarem com erro de parâmetro baseia-se na análise das medidas que apresentam os maiores resíduos normalizados. Porém, devido ao efeito de espalhamento do resíduo, não é possível garantir que a medida com o maior resíduo normalizado será uma das medidas incidentes ao ramo 
com erro de parâmetro. Assim, para reduzir a possibilidade de erro no processo de identificação de ramos suspeitos, propõe-se o seguinte procedimento: após o processamento de um estimador de estado convencional, determinam-se as medidas que apresentam resíduos normalizados maiores que um valor pré-estabelecido utilizado para detecção e identificação de EGs. Em seguida, associam-se tais medidas para as linhas de transmissão, ou ramos do modelo barra-ramo, que lhe são incidentes. A partir dessas associações, calcula-se o chamado Vetor Índice de Suspeita, através da seguinte expressão:

$I S(i)=\operatorname{MRE}(i) / T M(i)$,

para $i=1, \ldots, n l$, sendo: $n l$ o número de ramos do sistema em análise, $I S(i)$ o índice de suspeita $(I S)$ de o ramo $i$ estar com EPs; MRE(i) o número de medidas, com resíduos normalizados maior que um valor pré-estabelecido, que relacionam o ramo $i$ (medidas de fluxo no ramo $i$ ou medidas de injeção em uma das barras terminais desse ramo); e $T M(i)$ o número total de medidas disponíveis que relacionam o ramo $i$.

Todos os ramos com $I S \geq 0$ serão considerados suspeitos de estarem com EPs. Porém, dar-se-á inicio a estimação de parâmetros pelos ramos com $I S \geq 0,50$, pois são os ramos com maior probabilidade de estarem com erros.

Observe que o procedimento proposto fornece uma lista de ramos suspeitos de estarem com EPs.

\subsubsection{Fase 2: Estimação dos Parâmetros Suspeitos}

Nesta fase estimam-se os parâmetros identificados como suspeitos na fase 1, ou seja, as condutâncias série, susceptâncias série e susceptâncias shunt dos ramos identificados como suspeitos. Para isso, utiliza-se um estimador de estado e parâmetros baseado nas equações normais, que aumenta o vetor de variáveis de estado para inclusão dos parâmetros suspeitos.

Vale destacar que o estimador de estado e parâmetro é também uma proposta deste trabalho. Sua formulação será apresentada detalhadamente na seção 4.3.2.1.

A idéia que norteia a Fase 2 consiste em utilizar medidas de diversas amostras e estimar as variáveis de estado e os parâmetros suspeitos de forma seqüencial. Em linhas gerais, o procedimento proposto é o seguinte: 
Instante 11 (primeira amostra de medidas a ser processada): A partir das medidas disponíveis neste instante, estimam-se as variáveis de estado convencionais (tensões complexas nas barras do sistema) $\left(\underline{x}^{t l}\right)$ e os parâmetros suspeitos $(\underline{P})$, através do estimador de estado e parâmetros aqui proposto. Observe que, nesse instante, o estimador será processado utilizando, como condição inicial para os parâmetros suspeitos, os valores dos parâmetros disponíveis no banco de dados. Por outro lado, todas as variáveis de estado convencionais serão inicializadas no flat-start (magnitude de tensão igual a 1 p.u. e ângulo de fase igual a 0 rad. para todas as barras do sistema).

Instante t2: Através das medidas ora disponíveis, estimam-se, via estimador de estado e parâmetros, as variáveis de estado $\left(\underline{x}^{t 2}\right)$ e os parâmetros suspeitos $(\underline{P})$. Em termos de condição inicial, a diferença, em relação à condição adotada no instante anterior, e que os valores a serem utilizados, como condição inicial para as variáveis de estado $\left(\underline{x}^{t 2}\right)$ e para os parâmetros suspeitos, serão aqueles estimados no instante anterior $\left(\underline{x}^{t 2} \mathrm{e} \underline{\hat{P}}^{t 1}\right)$. Lembremonos de que estamos considerando a estimação de parâmetros que permanecem praticamente constantes por um determinado período de tempo. Isto é, durante o intervalo de tempo em análise.

O critério de parada do procedimento proposto é a comparação da magnitude das correções dos parâmetros, obtidas em dois instantes de tempo consecutivos ( $\left.\Delta \underline{\hat{P}}=\left|\underline{\hat{P}}^{t(k)}-\underline{\hat{P}}^{t(k-1)}\right|\right)$, com uma tolerância pré-estabelecida. À medida que a magnitude da correção de um determinado parâmetro, obtido em dois instantes de tempo consecutivos ( $\left.\Delta \hat{P}_{i}=\left|\hat{P}_{i}^{t(k)}-\hat{P}_{i}^{t(k-1)}\right|\right)$, for menor que 0,01 , o correspondente parâmetro é removido do vetor de variáveis de estado. O procedimento termina quando as magnitudes, de todas as correções obtidas em dois instantes de tempo consecutivos, forem menores que 0,01.

Face ao exposto, esta fase consiste em ciclos consecutivos de estimações de estado e parâmetros, uma para cada amostra de medida, até que o critério de convergência supracitado seja alcançado. Observe que, a partir da segunda amostra, os valores utilizados como condição inicial para os parâmetros suspeitos são as estimativas obtidas na amostra anterior, permitindo, assim, a correção dos valores desses parâmetros.

O procedimento proposto pode ser considerado um filtro "adaptativo", uma vez que as estimativas dos parâmetros melhoram de uma amostra para outra. 


\subsubsection{Estimador de Estado e Parâmetros Proposto}

O estimador de estado e parâmetros proposto baseia-se nas equações normais. Conseqüentemente, a sua formulação é, de uma forma geral, a mesma do estimador de estado convencional, por mínimos quadrados ponderados, apresentado no capítulo 2. Porém, no estimador de estado proposto, o vetor de variáveis de estado convencional é aumentado para considerar os parâmetros a serem estimados. Em razão disto, temos agora um modelo aumentado e o vetor de variáveis de estado passa a ser chamado de vetor de variáveis de estado "aumentado".

Considerando o modelo aumentado, a equação não-linear que relaciona o vetor de medidas $Z$ e o vetor de estado aumentado $x_{\text {Aum }}$, torna-se:

$$
Z=h\left(x_{\text {Aum }}\right)+w
$$

onde: $Z$ : o vetor de medidas $(\mathrm{mx} 1)$;

$h($.$) : o vetor de funções não lineares, que relaciona as medidas com as variáveis de$ estado "aumentadas" a serem estimadas ( $\mathrm{mx} 1)$;

$x_{\text {Aum }}:$ o vetor de variáveis de estado aumentado $\left(\mathrm{n}_{\text {Aum }} \mathrm{x} 1\right)$;

$w \quad$ : o vetor dos erros nas medidas (mx1), que são considerados como variáveis aleatórias independentes, com distribuição Gaussiana de média zero e matriz de covariância $W$;

$n_{\text {Aum }}$ : número de variáveis de estado aumentadas, a serem estimadas;

$m$ : número de medidas disponível num determinado instante de tempo.

Para um sistema com "L" ramos e "nb" barras, temos " $n_{\text {Aum }}=2 n b-1+3 L$ " (sendo "nb" magnitudes de tensão, "nb-1" ângulos de tensão e "3L" parâmetros).

Através da teoia de mínimos quadrados ponderados, a melhor estimativa de $x_{\text {Aum }}$, aqui designado por $\hat{x}_{\text {Aum }}$, é obtida determinando-se o valor de $x_{\text {Aum }}$ que minimiza o índice $J\left(x_{\text {Aum }}\right)$, dado por:

$$
J\left(x_{\text {Aum }}\right)=\left[z-h\left(x_{\text {Aum }}\right)\right]^{-1} W^{-1}\left[z-h\left(x_{\text {Aum }}\right)\right] .
$$

Desta forma $\hat{x}_{\text {Aum }}$ é obtida de: 


$$
\frac{\partial J\left(x_{\text {Aum }}\right)}{\partial x_{\text {Aum }}}=H^{T}\left(x_{\text {Aum }}\right) W^{-1}\left[z-h\left(x_{\text {Aum }}\right)\right]=0,
$$

sendo $H\left(x_{\text {Aum }}\right)=\frac{\partial h\left(x_{\text {Aum }}\right)}{x_{\text {Aum }}}$.

A equação (4.4) leva ao seguinte processo iterativo:

$$
G\left(x_{\text {Aum }}^{k}\right)\left(x_{\text {Aum }}^{k+1}-x_{\text {Aum }}^{k}\right)=H^{T}\left(x_{\text {Aum }}^{k}\right) W^{-1}\left[Z-h\left(x_{\text {Aum }}^{k}\right)\right] \text {, }
$$

onde $x_{\text {Aum }}^{k}$ é o valor de $x_{\text {Aum }}$ na iteração k, e

$$
G\left(x_{\text {Aum }}^{k}\right)=H^{T}\left(x_{\text {Aum }}^{k}\right) W^{-1} H\left(x_{\text {Aum }}^{k}\right)
$$

é a matriz Ganho Aumentada $\left(G_{\text {Aum }}\right)$ na iteração k.

Vale lembrar que:

$$
x_{\text {Aum }}=\left[\begin{array}{ll}
x_{e} & x_{p}
\end{array}\right]^{T},
$$

sendo $x_{e}$ o vetor das variáveis de estado tradicionais (tensões complexas nas barras do sistema) e $x_{p}$ o vetor dos parâmetros a serem estimados (parâmetros suspeitos de estarem com erros). Logo:

$$
H\left(x_{\text {Aum }}\right)=\frac{\partial h\left(x_{\text {Aum }}\right)}{\partial x_{\text {Aum }}}=\left[\begin{array}{ll}
\frac{\partial h\left(x_{e}\right)}{\partial x_{e}} & \frac{\partial h\left(x_{p}\right)}{\partial x_{p}}
\end{array}\right] \Delta\left[\begin{array}{ll}
H_{e} & H_{p}
\end{array}\right]
$$

A solução do modelo aumentado através do processo iterativo apresentado acima, isto é, via a matriz $G_{A u m}$, possui as seguintes desvantagens:

1) Em razão de as sensibilidades $\frac{\partial h\left(x_{e}\right)}{\partial\left(x_{e}\right)}$ serem mais intensas que as sensibilidades $\frac{\partial h\left(x_{p}\right)}{\partial\left(x_{p}\right)}$, a matriz $G_{A u m}$, dada pela equação (4.6), torna-se mal condicionada;

2) Raramente o número de medidas disponíveis é suficiente para estimar o vetor de variáveis de estado aumentado, pois, teremos $Z$ medidas para estimar $2 n b-1+3 L$ variáveis de estado.

Para contornar esse problema, o estimador de estado e parâmetros prosto possibilita a estimação do vetor de variáveis de estado aumentado dividindo o problema em dois subproblemas, conforme apresentado a seguir.

Formulação do Estimador de Estado e Parâmetros Proposto 
Considerando a equação (4.7), o índice $J\left(x_{A u m}\right)$, dado pela equação (4.3), pode ser escrito da seguinte forma:

$$
J\left(x_{e}, x_{p}\right)=\left[z-h\left(x_{e}, x_{p}\right)\right]^{-1} W^{-1}\left[z-h\left(x_{e}, x_{p}\right)\right] .
$$

Assim, as condições para otimalidade de Primeira Ordem são:

$$
\begin{aligned}
& H_{e}^{T}\left(\hat{x}_{e}, \hat{x}_{p}\right) R^{-1}\left[z-h\left(\hat{x}_{e}, \hat{x}_{p}\right)\right]=0 \mathrm{e} \\
& H_{p}^{T}\left(\hat{x}_{e}, \hat{x}_{p}\right) R^{-1}\left[z-h\left(\hat{x}_{e}, \hat{x}_{p}\right)\right]=0 .
\end{aligned}
$$

O estimador de estado e parâmetros proposto neste trabalho resolve a equação (4.10) de forma simultânea, ou seja, os subproblemas (4.10a) e (4.10b) são resolvidos alternadamente, via o método iterativo de Newton Raphon ${ }^{14}$, isto é: na solução do subproblema (4.10a) são utilizados o valores atualizados de $x_{p}$; na solução do subproblema (4.10b) são utilizados o valores atualizados de $x_{e}$.

Observe que as matrizes ganho utilizadas na solução dos subsistemas (4.10a) e (4.10b) são, respectivamente, as matrizes $G_{e}$ e $G_{p}$, apresentadas a seguir:

$$
\begin{aligned}
& G_{e}=H_{e}^{T} W^{-1} H_{e} \\
& G_{p}=H_{p}^{T} W^{-1} H_{p}
\end{aligned}
$$

As vantagens da obtenção de $x_{\text {Aum }}$ através da solução simultânea dos subproblemas (4.10a) e (4.10b) são as seguintes:

1) Resolvendo de forma simultânea, as matrizes envolvidas no processo de solução apresentam condicionamento numérico melhor que a matriz $G_{\text {Aum }}$. Dessa forma, garantesse maior robustez numérica para o alogoritmo de solução. A seguir apresenta-se um exemplo numérico para caracterizar que as matrizes envolvidas no proceso de solução simultânea são melhores condionadas;

2) Resolvendo da forma proposta, preserva-se a redundância das medidas para estimação dos parâmetros do sistema, pois, o vetor $Z$ utilizado para solução do subproblema (4.10a) é o mesmo utilizado para solução do subproblema (4.10b). Assim, se existem $m$ medidas disponíveis numa determinada amostra, teremos uma relação de redundância global de

\footnotetext{
${ }^{14}$ No apêndice C apresenta-se de forma sucinta o método de Newton-Raphson.
} 
$\frac{m}{2 n b-1}$ para solução do subproblema (4.10a) e de $\frac{m}{3 L}$ para solução do subproblema (4.10b).

\section{Equacionamento}

A matriz Jacobiana de estado $\left(H_{e}\right)$ e a matriz Jacobiana de parâmetros $\left(H_{p}\right)$

contém as derivadas das funções referentes a: $\underline{z}=\left[\begin{array}{c}F P A \\ F P R \\ M I A \\ M I R \\ V\end{array}\right]$, onde "FPA", "FPR", "MIA",

"MIR" e "V" indicam, respectivamente, vetor de medidas de fluxo de potência ativa, o vetor de medidas de fluxo de potência reativa, vetor de medidas de injeção de potência ativa, vetor de medidas de injeção de potência reativa e vetor de medidas de magnitude de tensão, em relação a $\underline{x}_{e}=\left[\begin{array}{l}v \\ \theta\end{array}\right]$ e $\underline{x}_{p}=[p]$, em que “ $\underline{\theta}$ " e “ $\underline{v}$ ” são, respectivamente, os vetores dos ângulos e das magnitudes das tensões das barras do sistema; e " $p$ " o vetor dos parâmetros a serem estimados.

$$
H_{e}=\left[\begin{array}{ll}
H_{F P v} & H_{F P \theta} \\
H_{F Q v} & H_{F Q \theta} \\
H_{P v} & H_{P \theta} \\
H_{Q v} & H_{Q \theta} \\
H_{V v} & H_{V \theta}
\end{array}\right]=\left[\begin{array}{cc}
\frac{\partial F P A}{\partial v} & \frac{\partial F P A}{\partial \theta} \\
\frac{\partial F P R}{\partial v} & \frac{\partial F P R}{\partial \theta} \\
\frac{\partial M I A}{\partial v} & \frac{\partial M I A}{\partial \theta} \\
\frac{\partial M I R}{\partial v} & \frac{\partial M I R}{\partial \theta} \\
\frac{\partial V}{\partial v} & \frac{\partial V}{\partial \theta}
\end{array}\right]
$$




$$
H_{p}=\left[\begin{array}{c}
H_{F P p} \\
H_{F Q p} \\
H_{P p} \\
H_{Q p} \\
H_{V p}
\end{array}\right]=\left[\begin{array}{c}
\frac{\partial F P A}{\partial p} \\
\frac{\partial F P R}{\partial p} \\
\frac{\partial M I A}{\partial p} \\
\frac{\partial M I R}{\partial p} \\
\frac{\partial V}{\partial p}
\end{array}\right]
$$

Das equações de fluxo de potência, considerando o modelo $\Pi$ Equivalente generalizado, apresentado na Figura 4.1 (MONTICELLI, 1999), tem-se:

$$
\begin{aligned}
& F P A=P_{k m}=a_{k m}^{2} V_{k}^{2} g_{k m}-a_{k m} V_{k} a_{m k} V_{m} g_{k m} \cos \left(\theta_{k m}+\varphi_{k m}-\varphi_{m k}\right)-a_{k m} V_{k} a_{m k} V_{m} b_{k m} \operatorname{sen}\left(\theta_{k m}+\varphi_{k m}-\varphi_{m k}\right) \\
& F P R=Q_{k m}=-a_{k m}^{2} V_{k}^{2}\left(b_{k m}+b_{k m}^{s h}\right)+a_{k m} V_{k} a_{m k} V_{m} b_{k m} \cos \left(\theta_{k m}+\varphi_{k m}-\varphi_{m k}\right)-a_{k m} V_{K} a_{m k} V_{m} g_{k m} \operatorname{sen}\left(\theta_{k m}+\varphi_{k m}-\varphi_{m k}\right) \\
& M I A=P_{k}=V_{k} \sum_{m E k} V_{m}\left(G_{k m} \cos \theta_{k m}+B_{k m} \operatorname{sen} \theta_{k m}\right) \\
& M I A=Q_{k}=V_{k} \sum_{m E k} V_{m}\left(G_{k m} \operatorname{sen} \theta_{k m}-B_{k m} \cos \theta_{k m}\right)
\end{aligned}
$$

No caso de linhas de transmissão, $\quad a_{k m}=a_{m k}=1$ e $\varphi_{k m}=\varphi_{m k}=0$; para transformadores em-fase, com tap no lado da barra $k, y_{k m}^{s h}=y_{m k}^{s h}=0, \varphi_{k m}=\varphi_{m k}=0$, e $a_{m k}=1$; e para os defasadores puros, com regulador no lado da barra $k, y_{k m}^{s h}=y_{m k}^{s h}=0$, $a_{k m}=a_{m k}=1$, e $\varphi_{m k}=0 . G_{i j}+j B_{i j}$ é o elemento (i,j) da matriz de admitância de barras.

Logo, os elementos que compõem as matrizes Jacobianas $H_{e}$ e $H_{p}$ são os seguintes:

Elementos correspondentes às magnitudes das tensões nodais:

$$
\begin{aligned}
& \frac{\partial P_{k}}{\partial V_{k}}=V_{k} G_{k k}+\sum_{m E k} V_{m}\left(G_{k m} \cos \theta_{k m}+B_{k m} \operatorname{sen} \theta_{k m}\right) \\
& \frac{\partial P_{k}}{\partial V_{m}}=V_{k}\left(G_{k m} \cos \theta_{k m}+B_{k m} \operatorname{sen} \theta_{k m}\right)
\end{aligned}
$$




$$
\begin{aligned}
& \frac{\partial Q_{k}}{\partial V_{k}}=-V_{k} B_{k k}+V_{k} \sum_{m E k} V_{m}\left(G_{k m} \operatorname{sen} \theta_{k m}-B_{k m} \cos \theta_{k m}\right) \\
& \frac{\partial Q_{k}}{\partial V_{m}}=V_{k}\left(G_{k m} \operatorname{sen} \theta_{k m}-B_{k m} \cos \theta_{k m}\right) \\
& \frac{\partial P_{k m}}{\partial V_{k}}=2 a_{k m}^{2} V_{k} g_{k m}-a_{k m} a_{m k} V_{m} g_{k m} \cos \left(\theta_{k m}+\varphi_{k m}-\varphi_{m k}\right)-a_{k m} a_{m k} V_{m} b_{k m} \operatorname{sen}\left(\theta_{k m}+\varphi_{k m}-\varphi_{m k}\right) \\
& \frac{\partial P_{k m}}{\partial V_{m}}=-a_{k m} a_{m k} V_{k} g_{k m} \cos \left(\theta_{k m}+\varphi_{k m}-\varphi_{m k}\right)-a_{k m} a_{m k} V_{k} b_{k m} \operatorname{sen}\left(\theta_{k m}+\varphi_{k m}-\varphi_{m k}\right) \\
& \frac{\partial Q_{k m}}{\partial V_{k}}=-2 a_{k m}^{2} V_{k}\left(b_{k m}+b_{k m}^{b}\right)+a_{k m} a_{m k} V_{m} b_{k m} \cos \left(\theta_{k m}+\varphi_{k m}-\varphi_{m k}\right)-a_{k m} a_{m k} V_{m} g_{k m} \operatorname{sen}\left(\theta_{k m}+\varphi_{k n}\right. \\
& \frac{\partial Q_{k m}}{\partial V_{m}}=a_{k m} a_{m k} V_{k} b_{k m} \cos \left(\theta_{k m}+\varphi_{k m}-\varphi_{m k}\right)-a_{k m} a_{m k} V_{k} g_{k m} \operatorname{sen}\left(\theta_{k m}+\varphi_{k m}-\varphi_{m k}\right) \\
& \frac{\partial V_{k}}{\partial V_{k}}=1 \\
& \frac{\partial V_{m}}{\partial V_{m}}=1 \\
& \frac{\partial V_{k}}{\partial V_{m}}=0 \\
& \frac{\partial V_{k}}{V_{k}}=0 \\
&
\end{aligned}
$$

Elementos correspondentes aos ângulos de fase das tensões nodais:

$$
\begin{aligned}
& \frac{\partial P_{k}}{\partial \theta_{k}}=-V_{k}^{2} B_{k k}-V_{k} \sum_{m E k} V_{m}\left(G_{k m} \operatorname{sen} \theta_{k m}-B_{k m} \cos \theta_{k m}\right) \\
& \frac{\partial P_{k}}{\partial \theta_{m}}=-V_{k} V_{m} G_{k m} \operatorname{sen} \theta_{k m}-B_{k m} \cos \theta_{k m} \\
& \frac{\partial Q_{k}}{\partial \theta_{k}}=-V_{k}^{2} G_{k k}+V_{k} \sum_{m E k} V_{m}\left(G_{k m} \cos \theta_{k m}+B_{k m} \operatorname{sen} \theta_{k m}\right) \\
& \frac{\partial Q_{k}}{\partial \theta_{m}}=-V_{k} V_{m}\left(G_{k m} \cos \theta_{k m}+B_{k m} \operatorname{sen} \theta_{k m}\right)
\end{aligned}
$$




$$
\begin{aligned}
& \frac{\partial P_{k m}}{\partial \theta_{k}}=a_{k m} V_{k} a_{m k} V_{m} g_{k m} \operatorname{sen}\left(\theta_{k m}+\varphi_{k m}-\varphi_{m k}\right)-a_{k m} V_{k} a_{m k} V_{m} b_{k m} \cos \left(\theta_{k m}+\varphi_{k m}-\varphi_{m k}\right) \\
& \frac{\partial P_{k m}}{\partial \theta_{m}}=-a_{k m} V_{k} a_{m k} V_{m} g_{k m} \operatorname{sen}\left(\theta_{k m}+\varphi_{k m}-\varphi_{m k}\right)+a_{k m} V_{k} a_{m k} V_{m} b_{k m} \cos \left(\theta_{k m}+\varphi_{k m}-\varphi_{m k}\right) \\
& \frac{\partial Q_{k m}}{\partial \theta_{k}}=-a_{k m} V_{k} a_{m k} V_{m} b_{k m} \operatorname{sen}\left(\theta_{k m}+\varphi_{k m}-\varphi_{m k}\right)-a_{k m} V_{k} a_{m k} V_{m} g_{k m} \cos \left(\theta_{k m}+\varphi_{k m}-\varphi_{m k}\right) \\
& \frac{\partial Q_{k m}}{\partial \theta_{m}}=a_{k m} V_{k} a_{m k} V_{m} b_{k m} \operatorname{sen}\left(\theta_{k m}+\varphi_{k m}-\varphi_{m k}\right)+a_{k m} V_{k} a_{m k} V_{m} g_{k m} \cos \left(\theta_{k m}+\varphi_{k m}-\varphi_{m k}\right) \\
& \frac{\partial V_{k}}{\partial \theta_{m}}=0 \\
& \frac{\partial V_{k}}{\partial \theta_{k}}=0 \\
& \frac{\partial V_{m}}{\partial \theta_{k}}=0 \\
& \frac{\partial V_{m}}{\partial \theta_{m}}=0
\end{aligned}
$$

Elementos correspondentes as suceptâncias série:

$$
\begin{aligned}
\frac{\partial V_{k}}{\partial b_{k m}} & =0 \\
\frac{\partial V_{m}}{\partial b_{k m}} & =0 \\
\frac{\partial P_{k}}{\partial b_{k m}} & =-a_{k m} V_{k} a_{m k} V_{m} \operatorname{sen}\left(\theta_{k m}+\varphi_{k m}-\varphi_{m k}\right) \\
\frac{\partial P_{m}}{\partial b_{k m}} & =-a_{k m} V_{k} a_{m k} V_{m} \operatorname{sen}\left(\theta_{m k}+\varphi_{k m}-\varphi_{m k}\right) \\
\frac{\partial Q_{k}}{\partial b_{k m}} & =-a_{k m}^{2} V_{k}^{2}+a_{k m} V_{k} a_{m k} V_{m} \cos \left(\theta_{k m}+\varphi_{k m}-\varphi_{m k}\right) \\
\frac{\partial Q_{m}}{\partial b_{k m}} & =-a_{k m}^{2} V_{m}^{2}+a_{k m} V_{k} a_{m k} V_{m} \cos \left(\theta_{m k}+\varphi_{k m}-\varphi_{m k}\right)
\end{aligned}
$$




$$
\begin{aligned}
& \frac{\partial P_{k m}}{\partial b_{k m}}=-a_{k m} V_{k} a_{m k} V_{m} \operatorname{sen}\left(\theta_{k m}+\varphi_{k m}-\varphi_{m k}\right) \\
& \frac{\partial Q_{k m}}{\partial b_{k m}}=-a_{k m}^{2} V_{k}^{2}+a_{k m} V_{k} a_{m k} V_{m} \cos \left(\theta_{k m}+\varphi_{k m}-\varphi_{m k}\right)
\end{aligned}
$$

Elementos correspondentes às condutâncias série:

$$
\begin{aligned}
& \frac{\partial V_{k}}{\partial g_{k m}}=0 \\
& \frac{\partial V_{m}}{\partial g_{k m}}=0 \\
& \frac{\partial P_{k}}{\partial g_{k m}}=-a_{k m} V_{k} a_{m k} V_{m} \cos \left(\theta_{k m}+\varphi_{k m}-\varphi_{m k}\right)+a_{k m}^{2} V_{k}^{2} \\
& \frac{\partial P_{m}}{\partial g_{k m}}=-a_{k m} V_{k} a_{m k} V_{m} \cos \left(\theta_{m k}+\varphi_{m k}-\varphi_{m k}\right)+a_{k m}^{2} V_{m}^{2} \\
& \frac{\partial Q_{k}}{\partial g_{k m}}=-a_{k m} V_{K} a_{m k} V_{m} \operatorname{sen}\left(\theta_{k m}+\varphi_{k m}-\varphi_{m k}\right) \\
& \frac{\partial Q_{m}}{\partial g_{k m}}=-a_{k m} V_{K} a_{m k} V_{m} \operatorname{sen}\left(\theta_{m k}+\varphi_{k m}-\varphi_{m k}\right) \\
& \frac{\partial P_{k m}}{\partial g_{k m}}=a_{k m}^{2} V_{k}^{2}-a_{k m} V_{k} a_{m k} V_{m} \cos \left(\theta_{k m}+\varphi_{k m}-\varphi_{m k}\right) \\
& \frac{\partial Q_{k m}}{\partial g_{k m}}=-a_{k m} V_{K} a_{m k} V_{m} \operatorname{sen}\left(\theta_{k m}+\varphi_{k m}-\varphi_{m k}\right)
\end{aligned}
$$

Elementos correspondentes às suceptâncias shunt:

$$
\begin{aligned}
\frac{\partial V_{k}}{\partial b_{k m}^{\text {shunt }}} & =0 \\
\frac{\partial V_{m}}{\partial b_{k m}^{\text {shunt }}} & =0 \\
\frac{\partial P_{k}}{\partial b_{k m}^{\text {shunt }}} & =0
\end{aligned}
$$


$\frac{\partial P_{m}}{\partial b_{k m}^{\text {shunt }}}=0$

$\frac{\partial Q_{k}}{\partial b_{k m}^{\text {shunt }}}=-a_{k m}^{2} V_{k}^{2}$

$\frac{\partial Q_{m}}{\partial b_{k m}^{\text {shunt }}}=-a_{k m}^{2} V_{m}^{2}$

$\frac{\partial P_{k m}}{\partial b_{k m}^{\text {shunt }}}=0$

$\frac{\partial Q_{k m}}{\partial b_{k m}^{\text {shunt }}}=-a_{k m}^{2} V_{k}^{2}$

Assim, de uma forma mais detalhada, as matrizes Jacobianas $H_{e}$ e $H_{p}$ apresentam as seguintes estruturas:

$H_{e}=\left[\begin{array}{cccc}H_{F P v} & H_{F P \theta} \\ H_{F Q v} & H_{F Q \theta} \\ H_{P v} & H_{P \theta} \\ H_{Q v} & H_{Q \theta} \\ H_{V v} & H_{V \theta}\end{array}\right]=\left[\begin{array}{cccc}\frac{\partial P_{k m}}{\partial V_{k}} & \frac{\partial P_{k m}}{\partial V_{m}} & \frac{\partial P_{k m}}{\partial \theta_{k}} & \frac{\partial P_{k m}}{\partial \theta_{m}} \\ \frac{\partial Q_{k m}}{\partial V_{k}} & \frac{\partial Q_{k m}}{\partial V_{m}} & \frac{\partial Q_{k m}}{\partial \theta_{k}} & \frac{\partial Q_{k m}}{\partial \theta_{m}} \\ \frac{\partial P_{k}}{\partial V_{k}} & \frac{\partial P_{k}}{\partial V_{m}} & \frac{\partial P_{k}}{\partial \theta_{k}} & \frac{\partial P_{k}}{\partial \theta_{m}} \\ \frac{\partial Q_{k}}{\partial V_{k}} & \frac{\partial Q_{k}}{\partial V_{m}} & \frac{\partial Q_{k}}{\partial \theta_{k}} & \frac{\partial Q_{k}}{\partial \theta_{m}} \\ \frac{\partial V_{k}}{\partial V_{k}} & 0 & 0 & 0 \\ 0 & \frac{\partial V_{m}}{\partial V_{m}} & 0 & 0\end{array}\right]$ 


$$
H_{p}=\left[\begin{array}{c}
H_{F P p} \\
H_{F Q p} \\
H_{P p} \\
H_{Q p} \\
H_{V p}
\end{array}\right]=\left[\begin{array}{ccc}
\frac{\partial P_{k m}}{\partial g_{k m}} & \frac{\partial P_{k m}}{\partial b_{k m}} & \frac{\partial P_{k m}}{\partial b_{k m}^{\text {shunt }}} \\
\frac{\partial Q_{k m}}{\partial g_{k m}} & \frac{\partial Q_{k m}}{\partial b_{k m}} & \frac{\partial Q_{k m}}{\partial b_{k m}^{\text {shunt }}} \\
\frac{\partial P_{k}}{\partial g_{k m}} & \frac{\partial P_{k}}{\partial b_{k m}} & \frac{\partial P_{k}}{\partial b_{k m}^{\text {shunt }}} \\
\frac{\partial Q_{k}}{\partial g_{k m}} & \frac{\partial Q_{k}}{\partial b_{k m}} & \frac{\partial Q_{k}}{\partial b_{k m}^{\text {shunt }}} \\
0 & 0 & 0 \\
0 & 0 & 0
\end{array}\right]
$$

As matrizes Ganho $\left(G_{e}\right)$ e ( $\left.G_{p}\right)$, que serão utilizadas no processo de estimação de estado e parâmetros, possuem as seguintes formas:

$$
\begin{aligned}
G_{e} & \left.=\left[H_{e}\right]^{t}\right][W]\left[H_{e}\right]=\left[\begin{array}{cc}
G_{v} & G_{\theta v} \\
G_{v \theta} & G_{\theta}
\end{array}\right] \\
G_{p} & =\left[H_{p}\right]^{t}[W]\left[H_{p}\right]=\left[G_{p}\right]
\end{aligned}
$$

Onde:

$$
\begin{aligned}
& G_{v}=\left[H_{P v}\right]^{T}\left[W_{P}\right]\left[H_{P v}\right]+\left[H_{Q v}\right]^{T}\left[W_{Q}\right]\left[H_{Q v}\right]+\left[H_{V v}\right]^{T}\left[W_{V}\right]\left[H_{V v}\right] \\
& G_{v \theta}=\left[H_{P v}\right]^{T}\left[W_{P}\right]\left[H_{P \theta}\right]+\left[H_{Q v}\right]^{T}\left[W_{Q}\right]\left[H_{Q \theta}\right]+\left[H_{V v}\right]^{T}\left[W_{V}\right]\left[H_{V \theta}\right] \\
& G_{\theta v}=\left[H_{P \theta}\right]^{T}\left[W_{P}\right]\left[H_{P V}\right]+\left[H_{Q \theta}\right]^{T}\left[W_{Q}\right]\left[H_{Q V}\right]+\left[H_{V \theta}\right]^{T}\left[W_{V}\right]\left[H_{V v}\right] \\
& G_{\theta}=\left[H_{P \theta}\right]^{T}\left[W_{P}\right]\left[H_{P \theta}\right]+\left[H_{Q \theta}\right]^{T}\left[W_{Q}\right]\left[H_{Q \theta}\right]+\left[H_{V \theta}\right]^{T}\left[W_{V}\right]\left[H_{V \theta}\right] \\
& G_{p}=\left[H_{P p}\right]^{T}\left[W_{P}\right]\left[H_{P p}\right]+\left[H_{Q p}\right]^{T}\left[W_{Q}\right]\left[H_{Q p}\right]+\left[H_{V p}\right]^{T}\left[W_{V}\right]\left[H_{V p}\right]
\end{aligned}
$$

As particularidades do algoritmo proposto são as seguintes:

$\checkmark$ a matriz Jacobiana $H_{e}$, da primeira meia iteração, é calculada para flat-start (V = 1 p.u. e $\theta=0$ rad. para todas as barras do sistema); 
$\checkmark$ os valores dos parâmetros a serem estimados, utilizados na primeira meia iteração, são os parâmetros disponíveis no banco de dados.

Com estas particularidades, os passos do algoritmo proposto são os seguintes:

\section{Algoritmo}

i) Fazer $v=0$ e inicialize o vetor de variáveis de estado convencionais no flat-start e o de parâmetros suspeitos com os valores disponíveis no banco de dados, isto é: $\underline{x}_{e}^{0}=\left[\begin{array}{l}\underline{v} \\ \underline{\theta}\end{array}\right]=\left[\begin{array}{l}\underline{1} \\ \underline{0}\end{array}\right]$ e $\underline{x}_{p}{ }^{0}=\left[\underline{p}_{(\text {Banco de dados })}\right]$

ii) Obtenha as correções nas variáveis de estado convencionais:

$$
\Delta \underline{x}_{e}{ }^{v}=\left[G_{e}\right]^{-1} \cdot H_{e}^{T} \cdot R^{-1} \cdot\left[z-h\left(\underline{x}_{e}{ }^{\nu}, \underline{x}_{p}{ }^{v}\right)\right]
$$

iii) Atualize o vetor de variáveis de estado convencionais: $\underline{x}_{e}^{v+1}=\underline{x}_{e}{ }^{v}+\Delta \underline{x}_{e}{ }^{v}$

iv) Obtenha as correções no vetor de parâmetros suspeitos:

$$
\Delta \underline{x}_{p}^{v}=\left[G_{p}\right]^{-1} \cdot H_{p}^{T} \cdot R^{-1} \cdot\left[z-h\left(\underline{x}_{e}^{v+1}, \underline{x}_{p}^{v}\right)\right]
$$

v) Atualize o vetor de parâmetros suspeitos: $\underline{x}_{p}{ }^{v+1}=\underline{x}_{p}{ }^{v}+\Delta \underline{x}_{p}{ }^{v}$

vi) Testar a convergência: se $\left|\Delta x_{e i}^{v}\right|_{M a x} e\left|\Delta x_{p i}^{v}\right|_{\text {Max }}$ forem menores que uma tolerância préestabelecida, o processo convergiu: $\hat{x}_{e}=x_{e}^{k+1}$ e $\hat{x}_{p}=x_{p}^{k+1}$. Caso contrário, faça $v=v+1 \mathrm{e}$ volte para o passo (ii).

\section{Exemplo}

Mostra-se, neste exemplo, que as matrizes ganho envolvidas na solução do subproblemas (4.10a) e (4.10b) são melhores condicionadas que a matriz $G_{\text {Aum }}$. Para isso, analisar-se-á o número de condição. das matrizes $G_{A u m}, G_{e}$ e $G_{p}$, associadas ao sistema de três barras ilustrado na Figura 4.2. Conforme apresentado no Apêndice D, matrizes com um número de condição elevado dizem-se mal condicionadas, e, matrizes com um número de condição baixo dizem-se bem condicionadas.

Os parâmetros desse sistema são apresentados na tabela 4.1. 


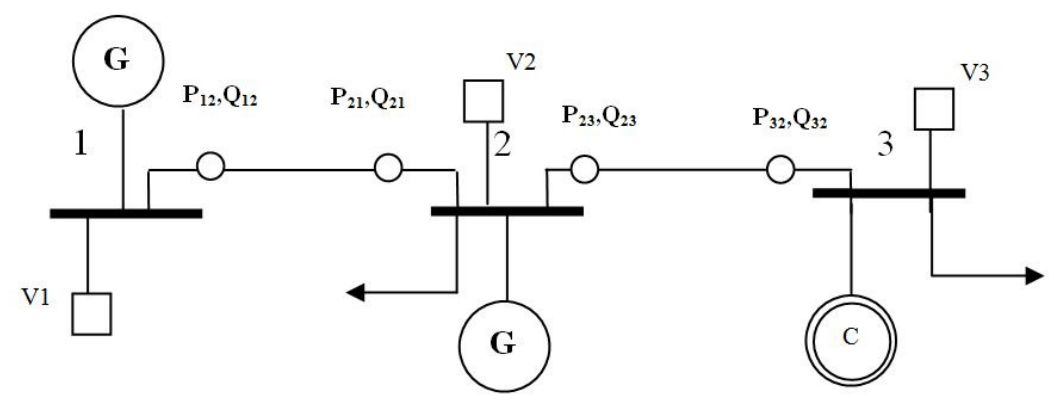

Onde:

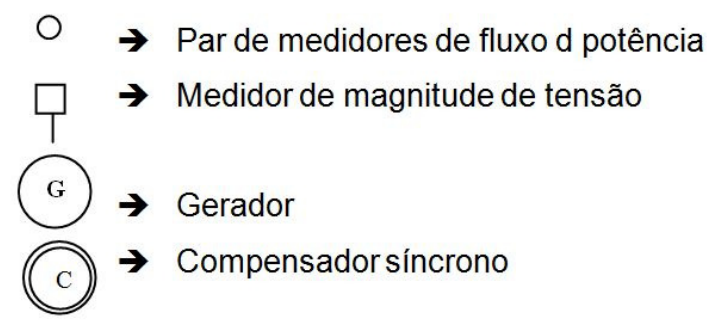

Figura 4.2 Sistema teste de 3 barras

Tabela 4.1: Parâmetros de linha do sistema de 3 barras.

\begin{tabular}{|c|c|c|c|}
\hline Parâmetros & Valor (p.u.) & Parâmetros & Valor (p.u.) \\
\hline $\mathrm{G}_{12}(\mathrm{pu})$ & 4.9991 & $\mathrm{G}_{23}(\mathrm{pu})$ & 1.1350 \\
\hline $\mathrm{B}_{12}(\mathrm{pu})$ & -15.263 & $\mathrm{~B}_{23}(\mathrm{pu})$ & -4.7818 \\
\hline$B_{12}^{s h}$ & 0.0264 & $B_{13}^{s h}$ & 0.0219 \\
\hline
\end{tabular}

As matrizes ganho obtidas para esse sistema são as seguintes:

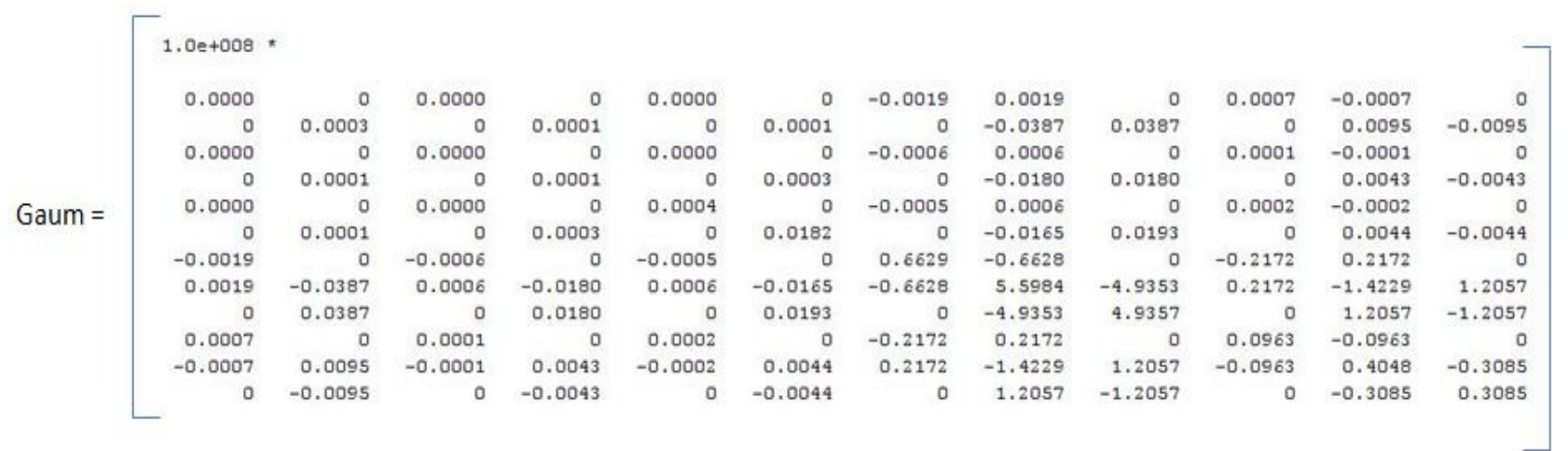




$$
\begin{aligned}
& \mathbf{G}_{\mathbf{e}}=\left[\begin{array}{rrrrr}
66294168 & -66275192 & 0 & 21719698 & 0 \\
-66275192 & 559842368 & -493527264 & -142286896 & 120567192 \\
0 & -493527264 & 493566752 & 120567192 & -120567192 \\
21719698 & -142286896 & 120567192 & 40483288 & -30851556 \\
0 & 120567192 & -120567192 & -30851556 & 30851556
\end{array}\right] \\
& \mathbf{G}_{\mathbf{p}}=\left[\begin{array}{rrrrrr}
1.0 e+006 & & & & & \\
0.0005 & 0 & 0.0002 & 0 & 0.0002 & 0 \\
0 & 0.0305 & 0 & 0.0141 & 0 & 0.0141 \\
0.0002 & 0 & 0.0001 & 0 & 0.0003 & 0 \\
0 & 0.0141 & 0 & 0.0068 & 0 & 0.0251 \\
0.0002 & 0 & 0.0003 & 0 & 0.0445 & 0 \\
0 & 0.0141 & 0 & 0.0251 & 0 & 1.8188
\end{array}\right]
\end{aligned}
$$

Condicionamento numérico:

- Cond $G_{\text {Aum }}=\left\|G_{\text {Aum }}\right\|\left\|G_{\text {Aum }}{ }^{-1}\right\|=6,0740 \times 10^{17}$;

- Cond $G_{e}=\left\|G_{e}\right\|\left\|G_{e}{ }^{-1}\right\|=3,3139 \times 10^{4}$;

- Cond $G_{p}=\left\|G_{p}\right\|\left\|G_{p}{ }^{-1}\right\|=1,0226 \times 10^{5}$.

Vale ressaltar que para melhorar ainda mais o condicionamento numérico das matrizes envolvidas no processo de estimação de estado e parâmetros, aplica-se a técnica de escalonamento de linhas e colunas descrita no Apêndice E. Para exemplificar a melhora que se obtem através dessa técnica, aplicando a mesma para matriz $G_{p}$ supracitda, obteve-se o número de condição 241,738 .

\section{Fase 3: Validação das estimativas dos parâmetros suspeitos}

Esta fase é necessária porque, em razão do efeito de espalhamento de resíduo, o processo de detecção e identificação de ramos suspeitos (Fase 1) pode falhar, isto é, pode classificar erroneamente um ramo como suspeito de estar com erro.

Para validar a estimativa do parâmetro de um dado ramo da rede, utiliza-se um estimador de estado convencional, por mínimos quadrados ponderados, que é processado considerando o valor estimado do parâmetro suspeito de estar com erro, e não o valor 
disponível no banco de dados. Se a estimativa estiver próxima do valor "verdadeiro" daquele parâmetro, o correspondente $I S$ diminuirá. Caso contrário, continuará o mesmo podendo, inclusive, aumentar.

\subsubsection{Algoritmo Geral para Depuração de Parâmetros Série e Shunt de Linhas de transmissão}

Nesta seção apresentam-se o algoritmo geral da metodologia proposta para validação de parâmetros série e shunt de linhas de transmissão e o correspondente fluxograma (Figura $4.3)$.

Em razão de a metodologia proposta consistir em três fases, o algoritmo proposto será também dividido em três fases e, além dos vetores $T M, M R E$ e $I S$, definidos na seção 4.3.1, o algoritmo faz uso de duas listas: Lista de Ramos Suspeitos (LRS) e Lista de Ramos cujos parâmetros já foram Estimados e Validados (LREV). Inicialmente ambas as listas estão vazias.

\section{FASE 1: Detecção e identificação de ramos suspeitos de estarem com EPS}

Passo 1: A partir de uma das amostras de medidas pré-selecionadas e do sistema em análise inicializar o vetor $T M$; vá para o próximo passo.

Passo 2: Executar o estimador de estado convencional por mínimos quadrados ponderados e obter os resíduos normalizados (serão utilizados os valores dos parâmetros disponíveis no banco de dados atual ou aqueles atualizados e validados no Passo 13); vá para o próximo passo.

Passo 3: Se houver medidas com resíduos normalizados, em módulo, maior que 3, ir para o Passo 4. Caso contrário, encerrar o processo (não existe suspeita de existência de EPs).

Passo 4: Inicializar o vetor $M R E$ e calcular o IS para cada um dos ramos do sistema (via equação (4.1)); vá para o próximo passo.

Passo 5: Montar uma lista ordenada de ramos suspeitos (LRS). O ramo que possuir o maior IS será o primeiro dessa lista, e assim sucessivamente (em caso de empate a ordenação será a partir do ramo incidente à medida com maior resíduo normalizado em módulo); vá para o próximo passo. 
Passo 6: Se existir pelo menos um ramo com $I S \geq 0,50$ vá para o passo 7. Caso contrário, vá para o Passo 9.

\section{FASE 2: Estimação de Parâmetros Suspeitos}

Passo 7: Selecionar o primeiro ramo $i-k$ da lista de suspeitos (LRS) e vá para o próximo passo.

Passo 8: Estimar os parâmetros do ramo $i-k$ (conforme procedimento apresentado na seção 4.3.2). Vá para o Passo 12.

Passo 9: Se a Lista LREV estiver vazia, voltar para o Passo 7. Caso contrário, vá para o próximo passo.

Passo 10 ${ }^{15}$ : Selecionar todos os ramos armazenados na lista LREV. Em seguida, estimar os parâmetros desses ramos (conforme procedimento apresentado na seção 4.3.2). Vá para o Passo 16.

Passo 11: Selecionar todos os ramos armazenados na lista LRS. Em seguida, estimar os parâmetros desses ramos (conforme procedimento apresentado na seção 4.3.2) e atualizar o banco de dados substituindo os valores dos parâmetros desses ramos disponíveis no banco de dados atual pelos valores aqui estimados. Fim de processamento.

Observação 4.1: Conforme apresentado na seção 4.3.2, nesta fase serão exigidas pelo menos duas das amostras pré-selecionadas de medidas.

\section{FASE 3: Validação das estimativas dos parâmetros suspeitos}

Passo 12: Estimar as variáveis de estado, através de um estimador de estado convencional por mínimos quadrados ponderados, considerando a mesma amostra de medidas processada no Passo 1 e os valores dos parâmetros disponíveis no banco de dados, com exceção dos parâmetros do ramo $i-k$. Para esses parâmetros devem ser considerados os valores estimados no Passo 8 . Vá para o próximo passo.

Passo 13: Calcular o novo IS para o ramo $i-k$ (equação 4.1). Se esse índice for menor que o calculado na Fase 1, vá para o passo 15. Caso contrário, vá para o próximo passo.

\footnotetext{
${ }^{15}$ Através deste passo é possível melhorar ainda mais as estimativas dos parâmetros com erro.
} 
Passo 14: Se existir mais algum ramo $i-k$, na lista de ramos suspeitos, que ainda não tenha sido analisado, selecione esse ramo e vá para o passo 8. Caso contrário, vá para o Passo 11.

Passo 15: Esvaziar a lista LRS ( $L R S=\phi$ ); atualizar o banco de dados substituindo os valores dos parâmetros do ramo $i$ - $k$, disponíveis no banco de dados atual, pelos valores estimados no Passo 8; armazenar o ramo $i-k$ na lista LREV (LREV — i-k ); volte para o Passo 1.

Passo 16: Estimar as variáveis de estado, através de um estimador de estado convencional por mínimos quadrados ponderados, considerando a mesma amostra de medidas processada no Passo 1 e os valores dos parâmetros disponíveis no banco de dados, com exceção dos parâmetros dos ramos armazenados na lista LREV. Para esses parâmetros devem ser utilizados os valores estimados no Passo 10. Calcule os resíduos normalizados para todas as medidas e vá para o próximo passo.

Passo 17: Se houver medidas com resíduos normalizados, em módulo, maior que 3, selecionar o próximo ramo $i-k$ da lista de suspeitos e voltar ao Passo 8. Caso contrário, vá para o próximo passo.

Passo 18: Atualizar o banco de dados substituindo os valores dos parâmetros dos ramos armazenados na lista LREV, disponíveis no banco de dados atual, pelos valores estimados no Passo 10. Fim de processamento. 


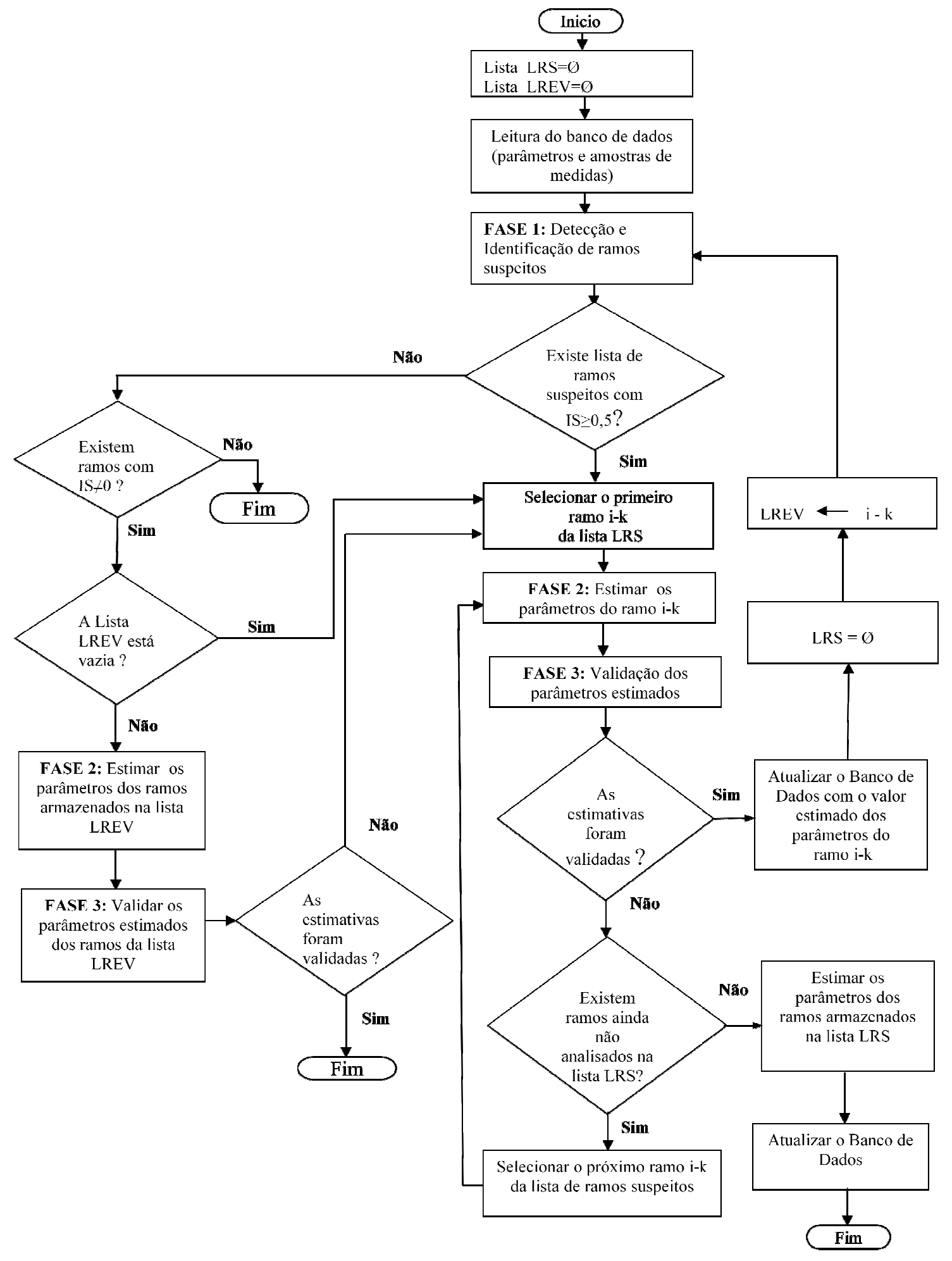

Figura 4.3 Fluxograma do algoritmo proposto 


\section{Capítulo 5}

\section{Testes Realizados e Análise dos Resultados}

Neste capítulo mostram-se os testes realizados para comprovar a eficiência da metodologia proposta, apresentada no capítulo 4. Foram utilizados os sistemas testes de 14, 30 e 57 barras do IEEE. Em todos os testes considerou-se:

- Erro de 5\% a 30\% em todos os parâmetros a serem estimados;

- Valores corretos para todos os parâmetros que não serão estimados;

- Os valores das medidas de cada amostra foram gerados a partir de um programa computacional desenvolvido em MATLAB (6.0). Esse programa adiciona, aos valores obtidos por um programa de fluxo de carga (ANAREDE), erros aleatórios de média zero e desvio padrão calculado através da equação (A.2), apresentada no Apêndice A;

- Para gerar uma série de amostras de medidas, que se ajustasse a realidade dos SEP, considerou-se uma evolução temporal das variáveis de estado do sistema, associada a um perfil de carga típico, para um período de tempo com intervalos de 5 minutos. A curva de carga de cada barra do sistema é composta por um percentual da carga total do sistema, submetido a uma flutuação aleatória, com distribuição normal de média zero e desvio padrão de $0,5 \%$ do valor do componente do fator de participação da carga em cada barra.

\subsection{Características de Implementação}

A metodologia proposta para depuração off-line de parâmetros de linhas de transmissão foi implantada em computador através da linguagem de programação Borland $\mathrm{C}++$ builder 
6.0. Os testes realizados foram executados em um computador Intel Core 2 Quad 2,4 GHz com 4 GB de memória RAM; e sistema operacional Windows 2000.

\subsection{Simulações com o sistema de 14 barras do IEEE}

Apresenta-se-nos na Figura 5.1, o sistema de 14 barras do IEEE utilizado para realização dos testes 1, 2, 3 e 4, que serão ilustrados a seguir. Em todas as 9 amostras que foram geradas para realização desses testes, considerou-se que estão disponíveis todas as medidas indicadas no sistema de medição encontrado na Figura 5.1. A Tabela 5.1 apresenta os valores que serão considerados como "corretos" das condutâncias, susceptâncias série e susceptâncias shunt do sistema. Todos os valores apresentados neste exemplo estão em p.u. (por unidade). 


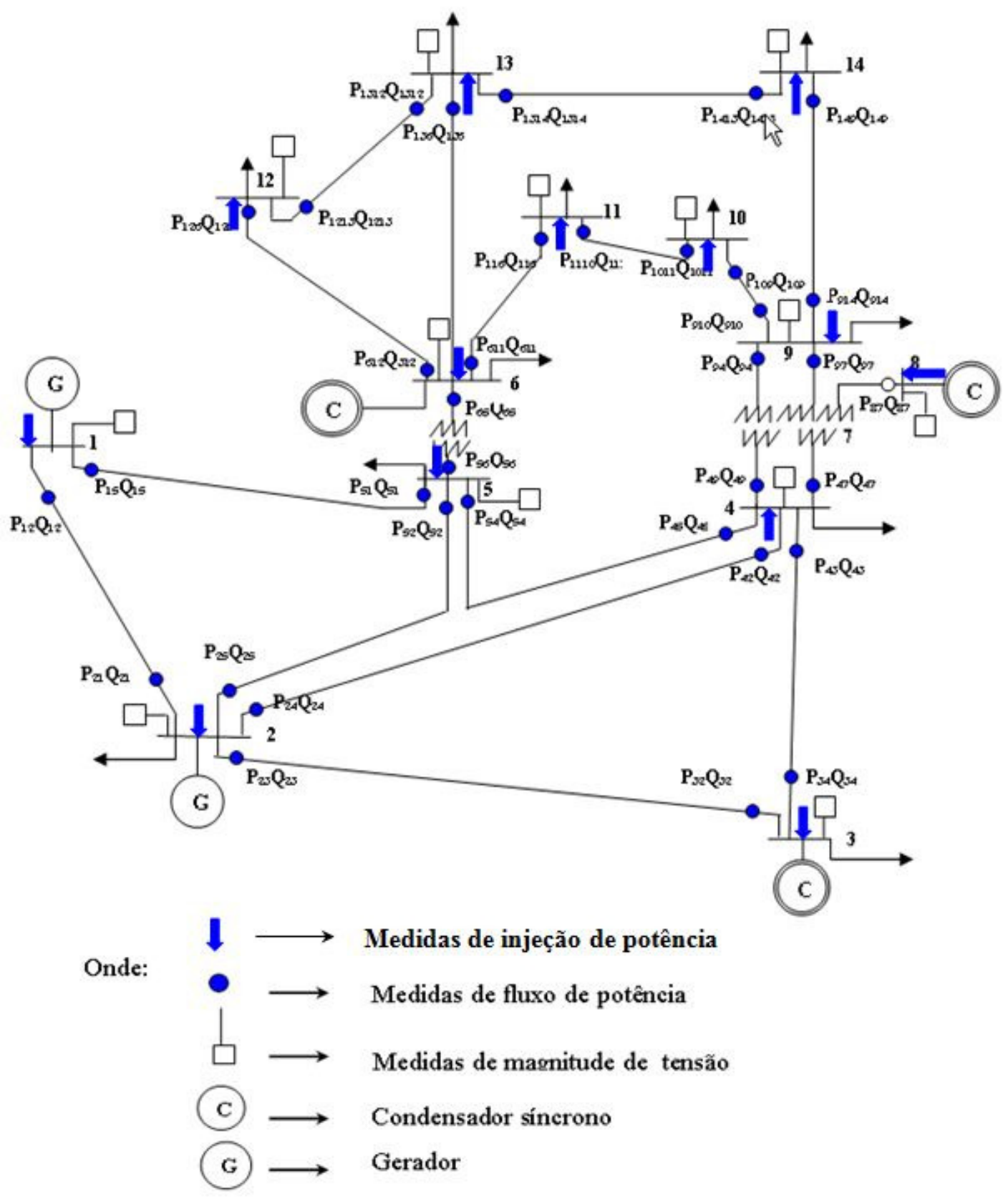

Figura 5.1: Sistema de 14 barras do IEEE associado a um conjunto redundante de medidas 
Tabela 5.1: Parâmetros de linha do sistema de 14 barras do IEEE utilizado nos testes

\begin{tabular}{|c|c|c|c|c|c|}
\hline Condutância & $\begin{array}{l}\text { Valores } \\
\text { corretos }\end{array}$ & Suceptância & $\begin{array}{l}\text { Valores } \\
\text { corretos }\end{array}$ & $\begin{array}{c}\text { Suceptância } \\
\text { shunt }\end{array}$ & $\begin{array}{l}\text { Valores } \\
\text { corretos }\end{array}$ \\
\hline $\mathbf{G}_{12}(\mathbf{p u})$ & 4,9991 & $\mathbf{B}_{12}(\mathbf{p u})$ & $-15,263$ & $\mathbf{B}^{\text {sh }}{ }_{12}(p u)$ & 0,0264 \\
\hline$G_{15}(p u)$ & 1,0258 & $\mathbf{B}_{15}(\mathbf{p u})$ & $-4,2349$ & $\mathrm{~B}_{15}^{\text {sh }}{ }_{15}(\mathrm{pu})$ & 0,0246 \\
\hline $\mathbf{G}_{23}(\mathbf{p u})$ & 1,1350 & $\mathbf{B}_{23}(\mathbf{p u})$ & $-4,7818$ & $\mathbf{B}^{\mathrm{sh}}{ }_{23}(\mathrm{pu})$ & 0,0219 \\
\hline $\mathbf{G}_{24}(\mathrm{pu})$ & 1,6860 & $\mathbf{B}_{24}(\mathbf{p u})$ & $-5,1158$ & $B^{\text {sh }}{ }_{24}(p u)$ & 0,0170 \\
\hline $\mathbf{G}_{25}(\mathbf{p u})$ & 1,7011 & $\mathbf{B}_{25}(\mathbf{p u})$ & $\begin{array}{l}-5,1939 \\
\end{array}$ & $\mathrm{~B}^{\mathrm{sh}}{ }_{25}(\mathrm{pu})$ & 0,0173 \\
\hline$G_{34}(p u)$ & 1,9859 & $\mathbf{B}_{34}(\mathbf{p u})$ & $-5,0688$ & $\mathbf{B}_{34}^{\text {sh }}(\mathbf{p u})$ & 0,0064 \\
\hline $\mathbf{G}_{45}(\mathbf{p u})$ & 6,8409 & $\mathbf{B}_{45}(\mathbf{p u})$ & $-21,578$ & $\mathrm{~B}^{\mathrm{sh}}{ }_{45}(\mathrm{pu})$ & 0 \\
\hline $\mathbf{G}_{47}(\mathbf{p u})$ & 0 & $\mathbf{B}_{47}(\mathbf{p u})$ & $-4,7819$ & $\mathbf{B}^{\mathrm{sh}}{ }_{47}(\mathbf{p u})$ & 0 \\
\hline $\mathbf{G}_{49}(\mathbf{p u})$ & 0 & $\mathbf{B}_{49}(\mathbf{p u})$ & $-1,7979$ & $\mathrm{~B}^{\mathrm{sh}}{ }_{49}(\mathrm{pu})$ & 0 \\
\hline$G_{56}(p u)$ & 0 & $\mathbf{B}_{56}(\mathbf{p u})$ & $-3,9679$ & $\mathrm{~B}^{\mathrm{sh}}{ }_{56}(\mathrm{pu})$ & 0 \\
\hline $\mathbf{G}_{611}(\mathbf{p u})$ & 1,9550 & $\mathbf{B}_{611}(\mathbf{p u})$ & $-4,0940$ & $\mathrm{~B}^{\mathrm{sh}}{ }_{611}(\mathrm{pu})$ & 0 \\
\hline $\mathbf{G}_{612}(\mathbf{p u})$ & 1,5259 & $\mathbf{B}_{612}(\mathbf{p u})$ & $-3,1759$ & $\mathrm{~B}_{612}^{\mathrm{sh}}(\mathrm{pu})$ & 0 \\
\hline $\mathbf{G}_{613}(\mathbf{p u})$ & 3,0989 & $\mathbf{B}_{613}(\mathbf{p u})$ & $-6,1027$ & $\mathrm{~B}^{\mathrm{sh}}{ }_{613}(\mathrm{pu})$ & 0 \\
\hline $\mathbf{G}_{78}(\mathbf{p u})$ & 0 & $\mathbf{B}_{78}(\mathbf{p u})$ & $\begin{array}{l}-5,6769 \\
\end{array}$ & $\mathbf{B}^{\text {sh }}{ }_{78}(\mathbf{p u})$ & 0 \\
\hline $\mathbf{G}_{79}(\mathbf{p u})$ & 0 & $\mathbf{B}_{79}(\mathbf{p u})$ & $-9,0900$ & $\mathbf{B}_{\mathrm{h} 79}^{\mathrm{s}}(\mathbf{p u})$ & 0 \\
\hline $\mathbf{G}_{910}(\mathbf{p u})$ & 3,9020 & $\mathbf{B}_{910}(\mathbf{p u})$ & $-10,365$ & $\mathbf{B}_{910}^{\mathrm{sh}}(\mathrm{pu})$ & 0 \\
\hline $\mathbf{G}_{914}(\mathbf{p u})$ & 1,4240 & $\mathbf{B}_{914}(\mathbf{p u})$ & $-3,0290$ & $\mathrm{~B}^{\mathrm{sh}_{914}}(\mathrm{pu})$ & 0 \\
\hline $\mathrm{G}_{1011}(\mathrm{pu})$ & 1,8808 & $\overline{B_{1011}(p u)}$ & $-4,4029$ & $\mathrm{~B}^{\text {sh }}{ }_{1011}(\mathrm{pu})$ & 0 \\
\hline $\mathrm{G}_{1213}(\mathrm{pu})$ & 2,4890 & $\overline{B_{1213}(p u)}$ & $-2,2519$ & $\mathrm{~B}^{\mathrm{sh}}{ }_{1213}(\mathrm{pu})$ & 0 \\
\hline$G_{1314}(p u)$ & 1,1369 & $B_{1314}(p u)$ & $-2,3149$ & $\mathrm{~B}^{\mathrm{sh}}{ }_{1314}(\mathrm{pu})$ & 0 \\
\hline
\end{tabular}

\section{Teste 1: Erros nos parâmetros do ramo 2-5}

Simulou-se erro nos parâmetros da admitância série e susceptância shunt do ramo 25, adotando-se, respectivamente, os valores 1,9733 - j 5,5575 p.u. e 0,0223 p.u. quando os valores corretos são 1,7011 - j 5,1939 p.u. e 0,0173 p.u. Como resultado da aplicação da metodologia proposta temos (veja Fluxograma ilustrado na Figura 4.3, apresentada na página 83):

Fase 1: O resultado da aplicação desta fase é a lista de ramos suspeitos apresentada na Tabela 5.2. . As medidas em cada $M R E(i)$, com os maiores resíduos normalizados $\left(\mathrm{r}^{\mathrm{N}}\right)$, estão em azul na Coluna 4. O Ramo 2-5 é selecionado para ter seus parâmetros estimados.

Tabela 5.2: Lista de ramos suspeitos com $I S \neq 0$-Teste 1

\begin{tabular}{|c|c|c|c|c|c|}
\hline Ramo i & TM(i) & MRE(i) & Lista de Medidas & rN & IS(i) \\
\hline $2-5$ & 10 & 4 & $\mathbf{Q}_{2-5}, \mathrm{Q}_{5}, \mathrm{P}_{2-5}, \mathrm{P}_{5-2}$ & 33,8558 & 0,40 \\
\hline $1-5$ & 10 & 3 & $\mathbf{Q}_{5}, \mathrm{Q}_{1}, \mathbf{Q}_{1-5}$ & 9,6249 & 0,30 \\
\hline $3-4$ & 10 & 3 & $\mathbf{Q}_{4-3}, \mathrm{Q}_{3-4}, \mathbf{Q}_{4}$ & 4,6381 & 0,30 \\
\hline $2-4$ & 10 & 3 & $\mathbf{Q}_{2-4}, \mathbf{Q}_{4-2}, \mathbf{Q}_{4}$ & 13,1552 & 0,30 \\
\hline $4-7$ & 10 & 2 & $\mathbf{Q}_{4}, \mathbf{Q}_{4-7}$ & 3,2485 & 0,20 \\
\hline $4-5$ & 10 & 2 & $\mathbf{Q}_{5}, \mathbf{Q}_{4}$ & 9,6249 & 0,20 \\
\hline $2-3$ & 10 & 2 & $\mathbf{Q}_{3-2}, \mathbf{Q}_{2-3}$ & 3,2124 & 0,20 \\
\hline $1-2$ & 10 & 2 & $\mathbf{Q}_{2-1}, \mathrm{Q}_{1}$ & 3,8074 & 0,20 \\
\hline $5-6$ & 10 & 1 & $\mathbf{Q}_{5}$ & 9,6249 & 0,10 \\
\hline
\end{tabular}




\begin{tabular}{|c|c|c|c|c|c|}
\hline $4-9$ & 10 & 1 & $\mathbf{Q}_{4}$ & 3,2485 & 0,10 \\
\hline $6-11$ & 10 & 1 & $\mathbf{Q}_{6-11}$ & 3,5151 & 0,10 \\
\hline
\end{tabular}

Fase 2: Estima-se o valor 1,7093-j5.2268 pu, para admitância série do ramo 2-5 e 0,0176 pu para susceptância shunt. Conforme mencionado anteriormente, para aplicar a metodologia proposta foram geradas 9 amostras de medidas, da maneira apresentda na página 80. Porém, neste teste o critério de parada foi satisfeito na quarta amostra.

Fase 3: Validam-se as estimativas. Em seguida realizam-se as seguintes tarefas: a base de dados é atualizada, isto é, os valores disponíveis dos parâmetros do ramo 2-5 são substituídos pelos valores estimados; a lista de ramos suspeitos é esvaziada (LRS $=\phi)$; e o ramo 2-5 é armazenado na lista de ramos suspeitos cujos parâmetros já foram estimados e validados $(\mathrm{LREV} \leftarrow 2-5)$.

Fase 1': Não existem mais medidas com resíduo normalizado em módulo maior que 3. Fim da análise.

Na Tabela 5.3 apresentam-se os valores disponíveis no banco de dados (iniciais), os estimados e os corretos dos parâmetros do ramo 2-5, bem como a porcentagem de erros nos valores iniciais e a porcentagem de correção obtida através das estimativas obtidas. Observe que a metodologia proposta permitiu a correção do valor dos parâmetros de forma significativa, principalmente para susceptância série.

Tabela 5.3: Parâmetros estimados do Teste 1

\begin{tabular}{|c|c|c|c|c|c|}
\cline { 2 - 6 } \multicolumn{1}{c|}{} & Inicial & Estimado & Correto & $\begin{array}{c}\text { \% de erro nos } \\
\text { valores iniciais }\end{array}$ & $\begin{array}{c}\text { \% de erro nos valores } \\
\text { estimados }\end{array}$ \\
\hline $\mathrm{G}_{25}$ & 1,9733 & 1,6931 & 1,7011 & 16 & 0,4703 \\
\hline $\mathrm{B}_{25}$ & $-5,5575$ & $-5,1886$ & $-5,1939$ & 7 & 0,1020 \\
\hline$B_{25}^{\text {shunt }}$ & 0,0223 & 0,0171 & 0,0173 & 29 & 1,1560 \\
\hline
\end{tabular}

Para mostrar a melhora obtida nas estimativas das variáveis de estado convencionais, após a depuração dos valores dos parâmetros do ramo 2-5, na Tabela 5.4 apresentam-se as variáveis de estado estimadas a partir de um estimador de estado convencional, por mínimos quadrados ponderados, processado com os valores dos parâmetros disponíveis no banco de dados inicial (coluna 2) e com o banco de dados 
atualizado (coluna 3). Na coluna 4, da mesma tabela, estão os valores obtidos pelo mesmo estimador porém processado com os valores corretos dos parâmetros do sistema (apresentados na Tabela 5.1). Observe que os valores das colunas 3 e 4 apresentam valores muito próximos entre si.

Tabela 5.4 Comparação de estimativas - Teste 1

\begin{tabular}{|c|c|c|c|}
\hline & $\begin{array}{c}\text { Banco de Dados } \\
\text { Inicial }\end{array}$ & $\begin{array}{c}\text { Banco de Dados } \\
\text { Atualizado }\end{array}$ & Valores corretos \\
\hline$\theta 1(\mathrm{rad})$ & 0,0000 & 0,0000 & 0,0000 \\
\hline$\theta 2$ (rad) & $-0,0888$ & $-0,0870$ & $-0,0870$ \\
\hline$\theta 3$ (rad) & $-0,2244$ & $-0,2222$ & $-0,2223$ \\
\hline$\theta 4(\mathrm{rad})$ & $-0,1819$ & -01799 & $-0,1800$ \\
\hline$\theta 5(\mathrm{rad})$ & $-0,1541$ & $-0,1531$ & $-0,1531$ \\
\hline$\theta 6$ (rad) & $-0,2512$ & $-0,2480$ & $-0,2481$ \\
\hline$\theta 7(\mathrm{rad})$ & $-0,2359$ & $-0,2331$ & $-0,2332$ \\
\hline$\theta 8$ (rad) & $-0,2359$ & $-0,2331$ & $-0,2332$ \\
\hline$\theta 9$ (rad) & $-0,2640$ & $-0,2606$ & $-0,2608$ \\
\hline$\theta 10(\mathrm{rad})$ & $-0,2668$ & $-0,2634$ & $-0,2635$ \\
\hline$\theta 11(\mathrm{rad})$ & $-0,2613$ & $-0,2580$ & $-0,2581$ \\
\hline$\theta 12$ (rad) & $-0,2664$ & $-0,2629$ & $-0,2630$ \\
\hline$\theta 13(\mathrm{rad})$ & $-0,2678$ & $-0,2643$ & $-0,2644$ \\
\hline$\theta 14(\mathrm{rad})$ & $-0,2834$ & $-0,2797$ & $-0,2798$ \\
\hline V1 (pu) & 1,0518 & 1,0604 & 1,0602 \\
\hline V2 (pu) & 1,0369 & 1,0453 & 1,0451 \\
\hline V3 (pu) & 1,0018 & 1,0102 & 1,0100 \\
\hline V4 (pu) & 1,0092 & 1,0180 & 1,0178 \\
\hline V5 (pu) & 1,0112 & 1,0198 & 1,0196 \\
\hline V6 (pu) & 1,0609 & 1,0702 & 1,0700 \\
\hline V7 (pu) & 1,0527 & 1,0618 & 1,0616 \\
\hline V8 (pu) & 1,0815 & 1,0903 & 1,0901 \\
\hline V9 (pu) & 1,0470 & 1,0562 & 1,0560 \\
\hline V10 (pu) & 1,0420 & 1,0513 & 1,0510 \\
\hline V11(pu) & 1,0478 & 1,0572 & 1,0569 \\
\hline V12(pu) & 1,0459 & 1,0554 & 1,0552 \\
\hline V13(pu) & 1,0411 & 1,0506 & 1,0504 \\
\hline V14(pu) & 1,0263 & 1,0359 & 1,0356 \\
\hline
\end{tabular}

\section{Teste 2: Erros nos parâmetros de 2 ramos não adjacentes (1-2 e 3-4)}

Neste teste são simulados erros simultâneos nos parâmetros de dois ramos não adjacentes (ramos 1-2 e 3-4), ou seja, em ramos eletricamente distantes. O sistema de medição considerado neste teste é o mesmo apresentado na Figura 5.1, com a exceção das medidas de injeção de potência. Ou seja, são consideradas apenas as medidas de magnitude de tensão e de fluxo apresentadas naquela figura. Como resultado da aplicação da 
metodologia proposta temos (veja Fluxograma ilustrado na Figura 4.3 apresentada no capítulo 4). Observe que considerando apenas medidas de fluxo e de magnitude de tensão o processo de identificação de ramos suspeitos funciona melhor, em razão do menor espalhamento do resíduo:

Fase 1: Diversos ramos são identificados como suspeitos (na Tabela 5.5 listam-se os ramos da LRS com $I S \neq 0)$. As medidas em cada MRE(i), com os maiores resíduos normalizados $\left(\mathrm{r}^{\mathrm{N}}\right)$, estão em azul na Coluna 4. O Ramo 1-2 é selecionado para ter seus parâmetros estimados.

Tabela 5.5: Lista 1 de ramos suspeitos com $I S \neq 0 \quad$ - Teste 2

\begin{tabular}{|c|c|c|c|c|c|}
\hline Ramo i & TM(i) & MRE(i) & Lista de Medidas & rN & IS(i) \\
\hline $1-2$ & 6 & 4 & $\mathbf{Q}_{1-2}, \mathrm{P}_{1-2}, \mathrm{P}_{2-1}, \mathrm{Q}_{2-1}$ & 18,85 & 0,67 \\
\hline $1-5$ & 6 & 4 & $\mathbf{Q}_{5-1}, \mathrm{P}_{1-5}, \mathrm{P}_{5-1}, \mathrm{Q}_{1-5}$ & 30,84 & 0,67 \\
\hline $2-5$ & 6 & 3 & $\mathbf{Q}_{5-2}, \mathrm{P}_{5-2}, \mathrm{P}_{2-5}$ & 15,58 & 0,5 \\
\hline $2-3$ & 6 & 3 & $\mathbf{Q}_{3-2}, \mathrm{P}_{3-2}, \mathrm{P}_{2-3}$ & 20,21 & 0,5 \\
\hline $3-4$ & 6 & 3 & $\mathbf{Q}_{4-3}, \mathrm{P}_{4-3}, \mathrm{P}_{3-4}$ & 17,16 & 0,5 \\
\hline $2-4$ & 6 & 2 & $\mathbf{Q}_{4-2}, \mathrm{P}_{2-4}$ & 16,30 & 0,33 \\
\hline $5-6$ & 6 & 1 & $\mathbf{Q}_{6-5}$ & 10,57 & 0,17 \\
\hline $4-9$ & 6 & 1 & $\mathbf{Q}_{4-9}$ & 3,58 & 0,17 \\
\hline $7-9$ & 6 & 1 & $\mathbf{Q}_{7-9}$ & 3,13 & 0,17 \\
\hline $6-11$ & 6 & 1 & $\mathbf{Q}_{11-6}$ & 3,18 & 0,17 \\
\hline
\end{tabular}

Fase 2: O processo de estimação de estado e parâmetros é executado, considerando todas as nove amostras de medidas. Estima-se o valor 4,8657-j14,7560 pu, para admitância série do ramo 1-2 e 0,0267 pu para susceptância shunt.

Fase 3: Validam-se as estimativas obtidas na Fase 2. Em seguida realizam-se as seguintes tarefas: - atualização do banco de dados: os valores disponíveis dos parâmetros do ramo 1-2 são substituídos pelos valores estimados, e o ramo 1-2 é armazenado na lista $L R E V(L R E V$ $\leftarrow 1-2) ;-$ LRS $=\phi$.

Fase 1': Forma-se uma nova lista LRS (Tabela 5.6), o ramo 2-3 é selecionado para ter seus parâmetros estimados.

Tabela 5.6: Lista 2 de ramos suspeitos com $I S \neq 0$ - Teste 2

\begin{tabular}{|c|c|c|c|c|c|}
\hline Ramo i & TM(i) & MRE(i) & $\begin{array}{c}\text { Lista de } \\
\text { Medidas }\end{array}$ & rN & IS(i) \\
\hline $2-3$ & 6 & 4 & $\begin{array}{c}\mathbf{Q}_{3-2}, \mathrm{Q}_{2-3}, \mathrm{P}_{3-2}, \\
\mathrm{P}_{2-3}\end{array}$ & 25,49 & 0,67 \\
\hline $3-4$ & 6 & 4 & $\mathbf{Q}_{4-3}, \mathrm{P}_{4-3}, \mathrm{P}_{3-4}$, & 18,23 & 0,67 \\
\hline
\end{tabular}




\begin{tabular}{|c|c|c|c|c|c|}
\hline & & & $\mathbf{Q}_{3-4}$ & & \\
\hline $5-6$ & 6 & 1 & $\mathbf{Q}_{6-5}$ & 6,48 & 0,17 \\
\hline $2-4$ & 6 & 1 & $\mathbf{Q}_{4-2}$ & 10,93 & 0,17 \\
\hline $4-5$ & 6 & 1 & $\mathbf{Q}_{4-5}$ & 3,42 & 0,17 \\
\hline $2-5$ & 6 & 1 & $\mathbf{Q}_{5-2}$ & 7,92 & 0,17 \\
\hline
\end{tabular}

Fase 2': O processo de estimação de estado e parâmetros convergiu na quarta amostra.

Fase 3': As estimativas obtidas na Fase anterior não são validadas, pois, o novo IS obtido para ramo 2-3 continua igual ao obtido na Fase 1'. Conclui-se, então, que esse ramo foi erroneamente identificado como suspeito. Assim, o próximo ramo $i$ - $k$ da lista LRS (ramo 34) é selecionado para ter seus parâmetros estimados.

Fase 2": O processo de estimação de estado e parâmetros convergiu na quarta amostra.

Fase 3": Validam-se as estimativas obtidas na Fase 2". Em seguida realizam-se as seguintes tarefas: - atualização do banco de dados: os valores disponíveis dos parâmetros do ramo 3-4 são substituídos pelos valores estimados, e o ramo 3-4 é armazenado na lista LREV $(L R E V \longleftarrow 3-4) ;-$ LRS $=\phi$.

Fase 1'": Neste momento nenhum dos ramos apresenta $I S \geq 0,5$, porém existem alguns com $I S \neq 0$. A lista $L R E V$ não está vazia.

Fase 2"'(Passo 10): Os ramos armazenados na lista LREV (ramos 1-2 e 3-4) são selecionados para terem os seus parâmetros estimados simultaneamente. Em seguida, estimam-se os parâmetros desses ramos.

Fase 3"'(Passo 16): Validam-se as estimativas dos parâmetros dos ramos armazenados na lista LREV. Em seguida realizam-se as seguintes tarefas: - atualização do banco de dados: os valores disponíveis dos parâmetros dos ramos armazenados na lista LREV são substituídos pelos valores estimados. Fim de processamento.

Os resultados obtidos para este teste estão expostos na Tabela 5.7. 
Tabela 5.7: Parâmetros estimados do Teste 2

\begin{tabular}{|c|c|c|c|c|c|}
\cline { 2 - 6 } \multicolumn{1}{c|}{} & Inicial & Estimado & Correto & $\begin{array}{c}\text { \% de erro nos valores } \\
\text { iniciais }\end{array}$ & $\begin{array}{c}\text { \% de erro nos valores } \\
\text { estimados }\end{array}$ \\
\hline $\mathrm{G}_{12}$ & 4,5492 & 4,9873 & 4,9991 & 9,0 & 0,2360 \\
\hline $\mathrm{B}_{12}$ & $-13,4314$ & $-15,1925$ & $-15,2630$ & 12,0 & 0,4619 \\
\hline$B_{12}^{\text {shunt }}$ & 0,0227 & 0,0262 & 0,0264 & 14,0 & 0,7575 \\
\hline $\mathrm{G}_{34}$ & 1,5093 & 1,9823 & 1,9859 & 24,0 & 0,1812 \\
\hline $\mathrm{B}_{34}$ & $-5,6264$ & $-5,0665$ & $-5,0688$ & 11,0 & 0,0454 \\
\hline$B_{34}^{\text {shunt }}$ & 0,0068 & 0,0062 & 0,0064 & 6,0 & 3,1250 \\
\hline
\end{tabular}

Na sexta coluna da Tabela 5.7 mostra-se, mais uma vez, que a metodologia proposta permite uma correção bastante significativa dos valores dos parâmetros de linhas de transmissão.

Da mesma forma que a Tabela 5.4, a Tabela 5.8 apresenta as estimativas das variáveis de estado convencionais obtidas por um estimador de estado convencional, por mínimos quadrados ponderados, nas seguintes condições: Coluna 2: através dos valores dos parâmetros disponíveis na base de dados inicial (com erros nos parâmetros dos ramos 1-2 e 3-4); Coluna 3: através dos valores dos parâmetros disponíveis no banco de dados atualizado (com os valores estimados dos parâmetros dos ramos 1-2 e 3-4); Coluna 4: com os valores corretos de todos os parâmetros do sistema (apresentados na Tabela 5.1). Observe que são bem próximos as estimativas apresentadas nas colunas 3 e 4, da Tabela 5.8 .

Tabela 5.8 Comparação de estimativas - Teste 2

\begin{tabular}{|c|c|c|c|}
\hline & $\begin{array}{c}\text { Banco de Dados } \\
\text { Inicial }\end{array}$ & $\begin{array}{c}\text { Banco de Dados } \\
\text { Atualizado }\end{array}$ & $\begin{array}{c}\text { Valores } \\
\text { corretos }\end{array}$ \\
\hline $\boldsymbol{\theta 1}(\mathbf{r a d})$ & 0,0000 & 0,0000 & 0,0000 \\
\hline $\boldsymbol{\theta 2}$ (rad) & $-0,0950$ & $-0,0873$ & $-0,0870$ \\
\hline $\boldsymbol{\theta 3}(\mathbf{r a d})$ & $-0,2269$ & $-0,2224$ & $-0,2223$ \\
\hline $\boldsymbol{\theta 4}(\mathbf{r a d})$ & $-0,1853$ & $-0,1801$ & $-0,1800$ \\
\hline $\boldsymbol{\theta 5}$ (rad) & $-0,1580$ & $-0,1533$ & $-0,1531$ \\
\hline $\boldsymbol{\theta 6}(\mathbf{r a d})$ & $-0,2523$ & $-0,2482$ & $-0,2481$ \\
\hline $\boldsymbol{\theta 7}(\mathbf{r a d})$ & $-0,2377$ & $-0,2333$ & $-0,2332$ \\
\hline $\boldsymbol{\theta 8}(\mathbf{r a d})$ & $-0,2377$ & $-0,2333$ & $-0,2332$ \\
\hline $\boldsymbol{\theta 9}$ (rad) & $-0,2649$ & $-0,2608$ & $-0,2608$ \\
\hline $\boldsymbol{\theta 1 0}(\mathbf{r a d )}$ & $-0,2676$ & $-0,2636$ & $-0,2635$ \\
\hline $\boldsymbol{\theta 1 1}(\mathbf{r a d})$ & $-0,2622$ & $-0,2582$ & $-0,2581$ \\
\hline $\boldsymbol{\theta 1 2}(\mathbf{r a d})$ & $-0,2670$ & $-0,2631$ & $-0,2630$ \\
\hline $\boldsymbol{\theta 1 3}(\mathbf{r a d})$ & $-0,2684$ & $-0,2645$ & $-0,2644$ \\
\hline $\boldsymbol{\theta 1 4}(\mathbf{r a d})$ & $-0,2837$ & $-0,2798$ & $-0,2798$ \\
\hline $\mathbf{V 1}(\mathbf{p u})$ & 1,0670 & 1,0604 & 1,0602 \\
\hline & & &
\end{tabular}




\begin{tabular}{|c|c|c|c|}
\hline $\mathbf{V 2}(\mathbf{p u})$ & 1,0503 & 1,0453 & 1,0451 \\
\hline $\mathbf{V 3}(\mathbf{p u})$ & 1,0180 & 1,0103 & 1,0100 \\
\hline $\mathbf{V 4}(\mathbf{p u})$ & 1,0228 & 1,0180 & 1,0178 \\
\hline $\mathbf{V 5}(\mathbf{p u})$ & 1,0249 & 1,0198 & 1,0196 \\
\hline $\mathbf{V 6}(\mathbf{p u})$ & 1,0756 & 1,0703 & 1,0700 \\
\hline $\mathbf{V 7}(\mathbf{p u})$ & 1,0669 & 1,0619 & 1,0616 \\
\hline $\mathbf{V 8}(\mathbf{p u})$ & 1,0952 & 1,0904 & 1,0901 \\
\hline $\mathbf{V 9}(\mathbf{p u})$ & 1,0614 & 1,0562 & 1,0560 \\
\hline $\mathbf{V 1 0}(\mathbf{p u})$ & 1,0565 & 1,0513 & 1,0510 \\
\hline $\mathbf{V 1 1}(\mathbf{p u})$ & 1,0624 & 1,0572 & 1,0569 \\
\hline $\mathbf{V 1 2}(\mathbf{p u})$ & 1,0608 & 1,0554 & 1,0552 \\
\hline $\mathbf{V 1 3}(\mathbf{p u})$ & 1,0560 & 1,0506 & 1,0504 \\
\hline $\mathbf{V 1 4}(\mathbf{p u})$ & 1,0412 & 1,0359 & 1,0356 \\
\hline
\end{tabular}

\section{Teste 3: Erros nos parâmetros de 2 ramos adjacentes (1-2 e 2-3)}

Erros múltiplos de parâmetros na condutância e susceptância series além da suceptância shunt dos ramos 1-2 e 2-3 são simulados Neste teste são consideradas todas as medidas ilustradas na Figura 5.1 e são simulados erros nos parâmetros de dois ramos adjacentes. Vale lembrar que, devido ao efeito de espalhamento de resíduo, o tratamento de EPs em ramos adjacentes torna-se bastante complicado (ZHU; ABUR, 2006), Como resultado da aplicação da metodologia proposta temos (veja Fluxograma ilustrado na Figura 4.3, apresentada no capitulo 4):

Fase 1: Diversos ramos são identificados como suspeitos (na Tabela 5.9 listam-se os ramos da LRS com $I S \neq 0)$.

Tabela 5.9: Lista 1 de ramos suspeitos com $I S \neq 0$-Teste 3

\begin{tabular}{|l|l|l|l|l|l|}
\hline \multicolumn{1}{|c|}{ Ramo i } & \multicolumn{1}{|c|}{ TM(i) } & \multicolumn{1}{c|}{ MRE(i) } & \multicolumn{1}{c|}{ Lista de Medidas } & \multicolumn{1}{c|}{ rN } & \multicolumn{1}{c|}{ IS(i) } \\
\hline $2-4$ & 10 & 7 & $\begin{array}{l}\mathbf{P}_{2}, \mathrm{Q}_{4-2}, \mathrm{P}_{4-2}, \mathrm{P}_{2-4}, \mathrm{P}_{4}, \mathrm{Q}_{2-4}, \\
\mathrm{Q}_{2}\end{array}$ & 24,3155 & 0,70 \\
\hline $2-3$ & 10 & 7 & $\begin{array}{l}\mathbf{P}_{2}, \mathrm{Q}_{2-3}, \mathrm{Q}_{3-2}, \mathrm{Q}_{2}, \mathrm{P}_{3-2}, \mathrm{P}_{2-3}, \\
\mathrm{P}_{3}\end{array}$ & 24,3155 & 0,70 \\
\hline $1-5$ & 10 & 6 & $\mathbf{Q}_{5-1}, \mathrm{Q}_{1-5}, \mathrm{Q}_{1}, \mathrm{P}_{5}, \mathrm{P}_{1-5}, \mathrm{P}_{5-1}$ & 18,2120 & 0,60 \\
\hline $2-5$ & 10 & 6 & $\mathbf{P}_{2}, \mathrm{Q}_{5-2}, \mathrm{P}_{5-2}, \mathrm{Q}_{2-5}, \mathrm{P}_{5}, \mathrm{Q}_{2}$ & 24,3155 & 0,60 \\
\hline $1-2$ & 10 & 5 & $\mathbf{P}_{2}, \mathrm{Q}_{1}, \mathrm{Q}_{2-1}, \mathrm{Q}_{1-2}, \mathrm{Q}_{2}$ & 24,3155 & 0,50 \\
\hline $3-4$ & 10 & 4 & $\mathbf{Q}_{3-4}, \mathrm{P}_{3}, \mathrm{P}_{4}, \mathrm{Q}_{4-3}$ & 9,0038 & 0,40 \\
\hline $4-5$ & 10 & 2 & $\mathbf{P}_{5}, \mathrm{P}_{4}$ & 7,6489 & 0,20 \\
\hline $5-6$ & 10 & 1 & $\mathbf{P}_{5}$ & 7,6489 & 0,10 \\
\hline $4-9$ & 10 & 1 & $\mathbf{P}_{4}$ & 7,3873 & 0,10 \\
\hline $4-7$ & 10 & 1 & $\mathbf{P}_{4}$ & 7,3873 & 0,10 \\
\hline
\end{tabular}


Fase 2: O ramo 2-4 é selecionado. Estima-se, então, 1,7696-5.3001j para a admitância série e 0,0163 para suceptancia shunt daquele ramo.

Fase 3: A estimativa obtida na fase anterior não é validada. Assim, o próximo ramo $i-k$ da lista de suspeitos (ramo 2-3) é selecionado para ser estimado.

Fase 2': Estima-se, então, o valor 1,1382-j4,8185 pu para a estimativa da admitância série e 0,0210 p.u para suceptancia shunt daquele ramo. Nesta fase o critério de parada foi satisfeito na oitava amostra.

Fase 3': Valida-se a estimativa dos parâmetros do ramo 2-3. Em seguida realizam-se as seguintes tarefas: - atualização do banco de dados: o valor disponível da admitância série do ramo 2-3 é substituído pelo valor estimado, e o ramo 2-3 é armazenado na lista $L R E V$ $(L R E V \longleftarrow 2-3) ;-$ LRS $=\phi$.

Fase 1": Forma-se agora a lista de ramos suspeitos apresentada na Tabela 5.10.

Tabela 5.10: Lista 2 de ramos suspeitos com $I S \neq 0$ - Teste 3

\begin{tabular}{|l|l|l|l|l|l|}
\hline \multicolumn{1}{|c|}{ Ramo i } & \multicolumn{1}{|c|}{ TM(i) } & \multicolumn{1}{c|}{ MRE(i) } & \multicolumn{1}{c|}{ Lista de Medidas } & \multicolumn{1}{c|}{ rN } & \multicolumn{1}{c|}{ IS(i) } \\
\hline $1-5$ & 10 & 6 & $\mathbf{Q}_{5-1}, \mathrm{Q}_{1-5}, \mathrm{Q}_{1}, \mathrm{P}_{5}, \mathrm{P}_{1-5}, \mathrm{P}_{5-1}$ & 16,3333 & 0,60 \\
\hline $2-5$ & 10 & 6 & $\mathbf{P}_{2}, \mathrm{Q}_{5-2}, \mathrm{P}_{5-2}, \mathrm{Q}_{2-5}, \mathrm{P}_{5}, \mathrm{Q}_{2}$ & 15,7891 & 0,60 \\
\hline $2-4$ & 10 & 6 & $\mathbf{P}_{2}, \mathrm{Q}_{4-2}, \mathrm{P}_{4-2}, \mathrm{P}_{2-4}, \mathrm{P}_{4}, \mathrm{Q}_{2-4}$ & 15,7891 & 0,60 \\
\hline $1-2$ & 10 & 5 & $\mathbf{P}_{2}, \mathrm{Q}_{1}, \mathrm{P}_{2-1}, \mathrm{P}_{1-2}, \mathrm{Q}_{2}$ & 15,7891 & 0,50 \\
\hline $2-3$ & 10 & 3 & $\mathbf{P}_{2}, \mathrm{Q}_{2-3}, \mathrm{Q}_{3-2}$ & 15,7891 & 0,30 \\
\hline $4-5$ & 10 & 2 & $\mathbf{P}_{4}, \mathrm{P}_{5}$ & 4,1218 & 0,20 \\
\hline $4-9$ & 10 & 1 & $\mathbf{P}_{4}$ & 4,1218 & 0,10 \\
\hline $5-6$ & 10 & 1 & $\mathbf{P}_{5}$ & 3,6000 & 0,10 \\
\hline $4-7$ & 10 & 1 & $\mathbf{P}_{4}$ & 4,1218 & 0,10 \\
\hline $3-4$ & 10 & 1 & $\mathbf{P}_{4}$ & 4,1218 & 0,10 \\
\hline
\end{tabular}

Fase 2": O ramo 1-5 é selecionado. Estima-se o valor 0,9690-j3,9911 pu da admitância série e $0,0271 \mathrm{pu}$ para a suceptancia shunt do ramo 1-5. Nesta fase o critério de parada foi satisfeito em três amostras.

Fase 3": A estimativa obtida na fase anterior não é validada. Assim, o próximo ramo $i-k$ da lista de suspeitos (ramo 2-5) é selecionado para ser estimado. 
Fase 2"': O ramo 2-5 é selecionado. Estima-se o valor 1,7551-j5,3223 pu da admitância série e 0,0165 pu para a suceptancia shunt do ramo 2-5. Nesta fase o critério de parada foi satisfeito em três amostras.

Fase 3"': A estimativa obtida na fase anterior não é validada. Assim, o próximo ramo $i-k$ da lista de suspeitos (ramo 2-4) é selecionado para ser estimado.

Fase 2'"': O ramo 2-4 é selecionado. Estima-se o valor 1,7224-j5,2225 pu da admitância série e 0,0166 pu para a suceptancia shunt do ramo 2-4. Nesta fase o critério de parada foi satisfeito em quatro amostras.

Fase 3"'": A estimativa obtida na fase anterior não é validada. Assim, o próximo ramo $i-k$ da lista de suspeitos (ramo 1-2) é selecionado para ser estimado.

Fase 2"'"': O ramo 1-2 é selecionado. Estima-se o valor 4,9918-j15,2124 pu da admitância série e 0,0265 pu para a suceptancia shunt do ramo 1-2. Nesta fase o critério de parada foi satisfeito em oito amostras.

Fase 3"'"': Valida-se a estimativa da admitância série do ramo 1-2. Em seguida realizam-se as seguintes tarefas: - atualização do banco de dados: o valor disponível do parâmetro do ramo 1-2 é substituído pelo valor estimado, e o ramo 1-2 é armazenado na lista LREV $(L R E V=\operatorname{ramo} 1-2 ;$ ramo 2-3); - LRS $=\phi$.

Fase 1'"'"': Neste momento nenhum dos ramos apresenta $I S \geq 0,5$, porém existem um com $I S \neq 0$, conforme indicado na lista LRS apresentada na Tabela 5.11.

Tabela 5.11: Lista 3 de ramos suspeitos com $I S \neq 0$ - Teste 3

\begin{tabular}{|c|c|c|c|c|c|}
\hline Ramo i & TM(i) & MRE(i) & Lista de Medidas & rN & IS(i) \\
\hline $2-3$ & 10 & 1 & $\mathbf{Q}_{2-3}$ & 3,5634 & 0,10 \\
\hline
\end{tabular}


Fase 2'"'"' (Passo 10): Os ramos armazenados na lista LREV (ramos 1-2 e 2-3) são selecionados. Em seguida, estimam-se os parâmetros desses ramos.

Fase 3"'"' (Passo 16): Validam-se as estimativas dos parâmetros dos ramos armazenados na lista $L R E V$. Em seguida realizam-se as seguintes tarefas: - atualização do banco de dados: os valores disponíveis dos parâmetros dos ramos armazenados na lista $L R E V$ são substituídos pelos valores estimados. Fim de processamento.

Os valores estimados estão expostos na terceira coluna da Tabela 5.12.

Tabela 5.12: Parâmetros estimados do Teste 3

\begin{tabular}{|c|c|c|c|c|c|}
\cline { 2 - 6 } \multicolumn{1}{c|}{} & Inicial & Estimado & Correto & $\begin{array}{c}\text { \% de erro nos valores } \\
\text { iniciais }\end{array}$ & $\begin{array}{c}\text { \% de erro nos valores } \\
\text { estimados }\end{array}$ \\
\hline $\mathrm{G}_{12}$ & 4,5492 & 4,9903 & 4,9991 & 9,0 & 0,1760 \\
\hline $\mathrm{B}_{12}$ & $-13,4314$ & $-15,2318$ & $-15,2630$ & 12,0 & 0,2044 \\
\hline$B_{12}^{\text {shunt }}$ & 0,0227 & 0,0263 & 0,0264 & 14,0 & 0,3787 \\
\hline $\mathrm{G}_{23}$ & 1,2825 & 1,1371 & 1,1350 & 13,0 & 0,1850 \\
\hline $\mathrm{B}_{23}$ & $-5,2600$ & $-4,8008$ & $-4,7818$ & 10,0 & 0,3973 \\
\hline$B_{23}^{\text {Shunt }}$ & 0,0241 & 0,0218 & 0,0219 & 10,0 & 0,4566 \\
\hline
\end{tabular}

Os resultados obtidos, apresentados na Tabela 5.12, mostram, mais uma vez, que a metodologia proposta conseguiu boas estimativas para os parâmetros, pois, partindo de um erro relativo de $9 \%$ para aqueles parâmetros, obteve um erro no ramo 1-2: $0.17 \%$ na condutância, e $0.20 \%$ na suceptância; ramo 2-3: $0.18 \%$ na condutância e $0.39 \%$ na suceptância.

Na Tabela 5.13 verifica-se que as estimativas obtidas, por um estimador de estado convencional, fazendo uso dos parâmetros estimados pela metodologia proposta, são bem próximos daqueles obtidos pelo mesmo estimador através dos valores corretos dos parâmetros.

Tabela 5.13 Comparação de estimativas - Teste 3

\begin{tabular}{|l|c|c|c|}
\hline & $\begin{array}{c}\text { Banco de Dados } \\
\text { Inicial }\end{array}$ & $\begin{array}{c}\text { Banco de Dados } \\
\text { Atualizado }\end{array}$ & Valores corretos \\
\hline $\boldsymbol{\theta 1}(\mathbf{r a d})$ & 0,0000 & 0,0000 & 0,0000 \\
\hline $\boldsymbol{\theta 2}(\mathbf{r a d})$ & $-0,0968$ & $-0,0872$ & $-0,0870$ \\
\hline $\boldsymbol{\theta 3}(\mathbf{r a d})$ & $-0,2265$ & $-0,2221$ & $-0,2223$ \\
\hline $\boldsymbol{\theta 4}(\mathbf{r a d})$ & $-0,1845$ & $-0,1800$ & $-0,1800$ \\
\hline
\end{tabular}




\begin{tabular}{|l|l|l|l|}
\hline $\boldsymbol{\theta 5}(\mathbf{r a d})$ & $-0,1578$ & $-0,1532$ & $-0,1531$ \\
\hline $\boldsymbol{\theta 6}(\mathbf{r a d})$ & $-0,2517$ & $-0,2482$ & $-0,2481$ \\
\hline $\boldsymbol{\theta 7}(\mathbf{r a d})$ & $-0,2370$ & $-0,2332$ & $-0,2332$ \\
\hline $\boldsymbol{\theta 8}(\mathbf{r a d})$ & $-0,2370$ & $-0,2332$ & $-0,2332$ \\
\hline $\boldsymbol{\theta 9}(\mathbf{r a d})$ & $-0,2642$ & $-0,2608$ & $-0,2608$ \\
\hline $\boldsymbol{\theta 1 0}(\mathbf{r a d})$ & $-0,0269$ & $-0,2635$ & $-0,2635$ \\
\hline $\boldsymbol{\theta 1 1}(\mathbf{r a d})$ & $-0,2616$ & $-0,2581$ & $-0,2581$ \\
\hline $\boldsymbol{\theta 1 2}(\mathbf{r a d})$ & $-0,2664$ & $-0,2630$ & $-0,2630$ \\
\hline $\boldsymbol{\theta 1 3}(\mathbf{r a d})$ & $-0,2678$ & $-0,2644$ & $-0,2644$ \\
\hline $\boldsymbol{\theta 1 4}(\mathbf{r a d})$ & $-0,2831$ & $-0,2798$ & $-0,2798$ \\
\hline $\mathbf{V 1}(\mathbf{p u})$ & 1,0650 & 1,0603 & 1,0602 \\
\hline $\mathbf{V 2}(\mathbf{p u})$ & 1,0474 & 1,0452 & 1,0451 \\
\hline $\mathbf{V 3}(\mathbf{p u})$ & 1,0134 & 1,0102 & 1,0100 \\
\hline $\mathbf{V 4}(\mathbf{p u})$ & 1,0216 & 1,0179 & 1,0178 \\
\hline $\mathbf{V 5}(\mathbf{p u})$ & 1,0234 & 1,0197 & 1,0196 \\
\hline $\mathbf{V 6}(\mathbf{p u})$ & 1,0739 & 1,0701 & 1,0700 \\
\hline $\mathbf{V 7}(\mathbf{p u})$ & 1,0654 & 1,0617 & 1,0616 \\
\hline $\mathbf{V 8}(\mathbf{p u})$ & 1,0938 & 1,0902 & 1,0901 \\
\hline $\mathbf{V 9}(\mathbf{p u})$ & 1,0599 & 1,0561 & 1,0560 \\
\hline $\mathbf{V 1 0}(\mathbf{p u})$ & 1,0550 & 1,0512 & 1,0510 \\
\hline $\mathbf{V 1 1}(\mathbf{p u})$ & 1,0609 & 1,0570 & 1,0569 \\
\hline $\mathbf{V 1 2}(\mathbf{p u})$ & 1,0592 & 1,0553 & 1,0552 \\
\hline $\mathbf{V 1 3}(\mathbf{p u})$ & 1,0544 & 1,0505 & 1,0504 \\
\hline $\mathbf{V 1 4}(\mathbf{p u})$ & 1,0392 & 1,0357 & 1,0356 \\
\hline & & & \\
\hline
\end{tabular}

\section{Teste 4: Erros nos parâmetros de 3 ramos (1-5,2-3,2-4)}

No Teste 3 foram simulados erros simultâneos nos parâmetros de dois ramos adjacentes considerando todas as medidas ilustradas na Figura 5.1. Neste teste são simulados erros nos parâmetros de três ramos (1-5, 2-3 e 2-4), sendo dois adjacentes (ramos $2-3$ e $2-4)$.

A seguir apresenta-se a tabelas com a lista de ramos suspeitos (Tabela 5.14), bem como com as estimativas obtidas para os parâmetros dos ramos 1-5, 2-3 e 2-4 (Tabela 5.15). A Tabela 5.16 segue o mesmo padrão das Tabelas 5.4, 5.8 e 5.13.

Tabela 5.14: Lista 1 de ramos suspeitos com $I S \neq 0$ (teste 4 )

\begin{tabular}{|l|l|l|l|l|l|}
\hline \multicolumn{1}{|c|}{ Ramo i } & \multicolumn{1}{|c|}{ TM(i) } & \multicolumn{1}{c|}{ MRE(i) } & \multicolumn{1}{c|}{ Lista de Medidas } & \multicolumn{1}{c|}{ rN } & \multicolumn{1}{c|}{ IS(i) } \\
\hline $4-5$ & 10 & 10 & $\begin{array}{l}\mathbf{Q}_{4}, \mathrm{Q}_{4-5}, \mathrm{P}_{4}, \mathrm{Q}_{5-4}, \mathrm{Q}_{5}, \mathrm{P}_{5}, \\
\mathrm{P}_{4-5}, \mathrm{P}_{5-4}, \mathrm{~V}_{5}, \mathrm{~V}_{4}\end{array}$ & 13,19 & 1,00 \\
\hline $2-4$ & 10 & 10 & $\begin{array}{l}\mathbf{Q}_{2-4}, \mathrm{Q}_{4-2}, \mathrm{Q}_{2}, \mathrm{P}_{2}, \mathrm{P}_{4-2}, \mathrm{P}_{2-4}, \\
\mathrm{Q}_{4}, \mathrm{P}_{4}, \mathrm{~V}_{4}, \mathrm{~V}_{2}\end{array}$ & 73,24 & 1,00 \\
\hline $3-4$ & 10 & 9 & $\begin{array}{l}\mathbf{Q}_{4-3}, \mathrm{Q}_{3-4}, \mathrm{P}_{4-3}, \mathrm{P}_{3-4}, \mathrm{Q}_{4}, \mathrm{P}_{4}, \\
\mathrm{~V}_{4}, \mathrm{~V}_{3}, \mathrm{Q}_{3}\end{array}$ & 26,40 & 0,90 \\
\hline
\end{tabular}




\begin{tabular}{|l|l|l|l|l|l|}
\hline $1-5$ & 10 & 9 & $\begin{array}{l}\mathbf{Q}_{1-5}, \mathrm{Q}_{1}, \mathrm{Q}_{5-1}, \mathrm{Q}_{5}, \mathrm{P}_{5}, \mathrm{P}_{5-1}, \\
\mathrm{P}_{1-5}, \mathrm{~V}_{5}\end{array}$ & 79,31 & 0,90 \\
\hline $2-3$ & 10 & 9 & $\begin{array}{l}\mathbf{Q}_{2-3}, \mathrm{Q}_{2}, \mathrm{Q}_{3-2}, \mathrm{P}_{2}, \mathrm{P}_{2-3}, \mathrm{P}_{3-2}, \\
\mathrm{~V}_{3}, \mathrm{~V}_{2}\end{array}$ & 37,98 & 0,90 \\
\hline $2-5$ & 10 & 8 & $\begin{array}{l}\mathbf{Q}_{2-5}, \mathrm{Q}_{2}, \mathrm{P}_{2}, \mathrm{Q}_{5}, \mathrm{P}_{5}, \mathrm{~V}_{5}, \\
\mathrm{~V}: 2, \mathrm{Q}_{5}, 5-2\end{array}$ & 77,71 & 0,80 \\
\hline $5-6$ & 10 & 7 & $\mathbf{Q}_{6-5}, \mathrm{Q}_{5}, \mathrm{P}_{5}, \mathrm{Q}_{6}, \mathrm{P}_{6}, \mathrm{~V}_{6}, \mathrm{~V}_{5}$ & 9,43 & 0,70 \\
\hline $4-7$ & 10 & 7 & $\begin{array}{l}\mathbf{Q}_{4}, \mathrm{P}_{4}, \mathrm{Q}_{4-7}, \mathrm{Q}_{7}, \mathrm{~V}_{7}, \mathrm{~V}_{4}, \mathrm{Q}_{7-} \\
4\end{array}$ & 13,19 & 0,70 \\
\hline $1-2$ & 10 & 7 & $\begin{array}{l}\mathbf{Q}_{1}, \mathrm{Q}_{1-2}, \mathrm{Q}_{2-1}, \mathrm{Q}_{2}, \mathrm{P}_{2}, \mathrm{~V}_{2}, \\
\mathrm{P}_{1},\end{array}$ & 48,96 & 0,70 \\
\hline $4-9$ & 10 & 6 & $\mathbf{Q}_{4}, \mathrm{P}_{4}, \mathrm{Q}_{9}, \mathrm{Q}_{4-9}, \mathrm{~V}_{9}, \mathrm{~V}_{4}$, & 13,19 & 0,60 \\
\hline $7-9$ & 10 & 6 & $\mathbf{Q}_{9}, \mathrm{Q}_{7-9}, \mathrm{Q}_{7}, \mathrm{~V}_{9}, \mathrm{~V}_{7}, \mathrm{Q}_{9-7}$ & 6,08 & 0,60 \\
\hline $6-12$ & 10 & 5 & $\mathbf{Q}_{6}, \mathrm{P}_{6}, \mathrm{~V}_{12}, \mathrm{Q}_{12-6}, \mathrm{Q}_{6}$ & 6,80 & 0,50 \\
\hline $6-11$ & 10 & 5 & $\mathbf{Q}_{6}, \mathrm{P}_{6}, \mathrm{~V}_{11}, \mathrm{~V}_{6}, \mathrm{P}_{11}$ & 6,80 & 0,50 \\
\hline $6-13$ & 10 & 4 & $\mathbf{Q}_{6}, \mathrm{~V}_{6}, \mathrm{P}_{6}$ & 6,80 & 0,40 \\
\hline $9-10$ & 10 & 3 & $\mathbf{Q}_{9}, \mathrm{~V}_{10}, \mathrm{~V}_{9}$ & 6,08 & 0,30 \\
\hline $9-14$ & 10 & 3 & $\mathbf{Q}_{9}, \mathrm{~V}_{14}, \mathrm{~V}_{9}$ & 6,085 & 0,30 \\
\hline $10-11$ & 10 & 3 & $\mathbf{V}_{11}, \mathrm{P}_{11}, \mathrm{~V}_{10}$ & 4,20 & 0,30 \\
\hline $7-8$ & 10 & 3 & $\mathbf{Q}_{7}, \mathrm{~V}_{7}, \mathrm{~V}_{8}$ & 4,81 & 0,30 \\
\hline $12-13$ & 10 & 2 & $\mathbf{V}_{13}, \mathrm{~V}_{12}$ & 4,15 & 0,20 \\
\hline $13-14$ & 10 & 2 & $\mathbf{V}_{13}, \mathrm{~V}_{12}$ & 4,15 & 0,20 \\
\hline
\end{tabular}

Tabela 5.15: Parâmetros estimados do Teste 4

\begin{tabular}{|c|c|c|c|c|c|}
\cline { 2 - 6 } \multicolumn{1}{c|}{} & Inicial* & Estimado & Correto & $\begin{array}{c}\text { \% de erro nos } \\
\text { valores iniciais }\end{array}$ & $\begin{array}{c}\text { \% de erro nos } \\
\text { valores estimados }\end{array}$ \\
\hline $\mathrm{G}_{1-5}$ & 1,2720 & 1,0282 & 1,0258 & 24,0 & 0,23 \\
\hline $\mathrm{B}_{1-5}$ & $-4,0232$ & $-4,2345$ & $-4,2349$ & 5,00 & 0,009 \\
\hline$B_{1-5}^{s h}$ & 0,0293 & 0,0245 & 0,0246 & 19,0 & 0,40 \\
\hline $\mathrm{G}_{2-3}$ & 1,2825 & 1,1427 & 1,1350 & 13,0 & 0,67 \\
\hline $\mathrm{B}_{2-3}$ & $-5,2600$ & $-4,7897$ & $-4,7818$ & 10,0 & 0,16 \\
\hline$B_{2-3}^{s h}$ & 0,0241 & 0,0218 & 0,0219 & 10,0 & 0,45 \\
\hline $\mathrm{G}_{24}$ & 1,9220 & 1,6974 & 1,6860 & 14,0 & 0,67 \\
\hline $\mathrm{B}_{24}$ & $-4,5019$ & $-5,1353$ & $-5,1158$ & 12,0 & 0,38 \\
\hline$B_{24}^{s h}$ & 0,0194 & 0,0173 & 0,0170 & 14,0 & 1,76 \\
\hline
\end{tabular}

Tabela 5.16 Comparação de estimativas - Teste 4

\begin{tabular}{|l|c|c|c|}
\hline & $\begin{array}{c}\text { Banco de Dados } \\
\text { Inicial }\end{array}$ & $\begin{array}{c}\text { Banco de Dados } \\
\text { Atualizado }\end{array}$ & Valores corretos \\
\hline $\boldsymbol{\theta 1}(\mathbf{r a d})$ & 0,0000 & 0,0000 & 0,0000 \\
\hline $\boldsymbol{\theta 2}(\mathbf{r a d})$ & $-0,0884$ & $-0,0871$ & $-0,0870$ \\
\hline $\boldsymbol{\theta 3}(\mathbf{r a d})$ & $-0,2219$ & $-0,2223$ & $-0,2223$ \\
\hline $\boldsymbol{\theta 4}(\mathbf{r a d})$ & $-0,1839$ & $-0,1799$ & $-0,1800$ \\
\hline $\boldsymbol{\theta 5}(\mathbf{r a d})$ & $-0,1568$ & $-0,1531$ & $-0,1531$ \\
\hline
\end{tabular}




\begin{tabular}{|c|c|c|c|}
\hline$\theta 6(\mathrm{rad})$ & $-0,2557$ & $-0,2481$ & $-0,2481$ \\
\hline$\theta 7($ rad $)$ & $-0,2399$ & $-0,2331$ & $-0,2332$ \\
\hline$\theta 8(\mathrm{rad})$ & $-0,2399$ & $-0,2331$ & $-0,2332$ \\
\hline$\theta 9$ (rad) & $-0,2689$ & $-0,2607$ & $-0,2608$ \\
\hline$\theta 10(\mathrm{rad})$ & $-0,2719$ & $-0,2635$ & $-0,2635$ \\
\hline$\theta 11(\mathrm{rad})$ & $-0,2662$ & $-0,2581$ & $-0,2581$ \\
\hline$\theta 12(\mathrm{rad})$ & $-0,2713$ & $-0,2630$ & $-0,2630$ \\
\hline$\theta 13(\mathrm{rad})$ & $-0,2728$ & $-0,2644$ & $-0,2644$ \\
\hline$\theta 14(\mathrm{rad})$ & $-0,2891$ & $-0,2798$ & $-0,2798$ \\
\hline V1 (pu) & 1,0390 & 1,0602 & 1,0602 \\
\hline V2 (pu) & 1,0201 & 1,0451 & 1,0451 \\
\hline V3 (pu) & 0,9841 & 1,0099 & 1,0100 \\
\hline V4 (pu) & 0,9881 & 1,0177 & 1,0178 \\
\hline V5 (pu) & 0,9903 & 1,0196 & 1,0196 \\
\hline V6 (pu) & 1,0389 & 1,0699 & 1,0700 \\
\hline V7 (pu) & 1,0308 & 1,0615 & 1,0616 \\
\hline V8 (pu) & 1,0598 & 1,0900 & 1,0901 \\
\hline V9 (pu) & 1,0248 & 1,0559 & 1,0560 \\
\hline $\mathrm{V10}(\mathrm{pu})$ & 1,0197 & 1,0510 & 1,0510 \\
\hline V11(pu) & 1,0256 & 1,0569 & 1,0569 \\
\hline V12(pu) & 1,0238 & 1,0551 & 1,0552 \\
\hline V13(pu) & 1,0190 & 1,0503 & 1,0504 \\
\hline V14(pu) & 1,0038 & 1,0355 & 1,0356 \\
\hline
\end{tabular}

\subsection{SIMULAÇÕES COM O SISTEMA DE 57 BARRAS DO IEEE}

Nos próximos testes será utilizado o sistema de 57 barras do IEEE associado ao conjunto de medidas apresentado na Figura 5.2 (para maior informação ver o site www.ee.washington.edu/research/pstca).

Para simplificar a apresentação dos resultados dos testes realizados com o sistema de 57 barras, os mesmos serão apresentados através de Tabelas, que seguem o mesmo formato das apresentadas na seção anterior. 


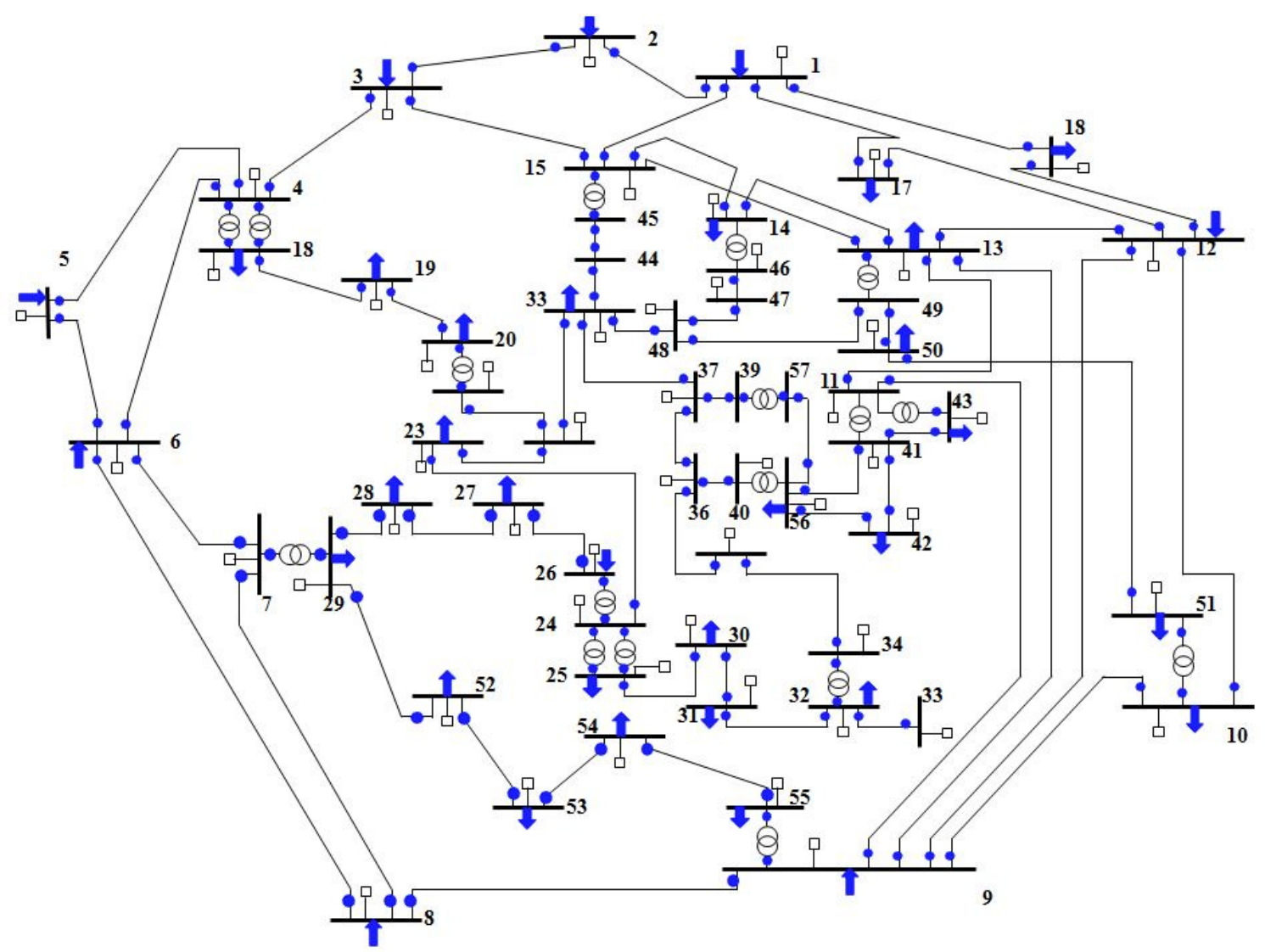

Figura 5.2: Sistema de 57 barras do IEEE

Teste 5: Erros nos parâmetros do ramo 12-16, sistema IEEE de 57 barras. (sistemas com medidas redundantes)

Simulou-se arbitrariamente um erro na admitância série e suceptância shunt do ramo 12-16, adotando-se o valor de 1,9969 - j 9,0195 p.u. e 0,0083 quando o valor correto seria de $2,5960-j 11 ., 7254$ p.u. e 0,0108 . Os resultados obtidos estão expostos nas tabelas a seguir.

Tabela 5.17: Lista de ramos suspeitos utilizando medidas de injeção, fluxo de potência e tensão com $I S \neq 0$ - teste 5

\begin{tabular}{|c|l|l|l|l|l|}
\hline Ramo $\mathbf{i}$ & \multicolumn{1}{|c|}{ TM(i) } & \multicolumn{1}{|c|}{ MRE(i) } & \multicolumn{1}{|c|}{ Lista de Medidas } & \multicolumn{1}{|c|}{ rN } & \multicolumn{1}{c|}{ IS(i) } \\
\hline $12-16$ & 10 & 5 & $\mathbf{Q}_{16-12}, \mathrm{P}_{12-16}, \mathrm{P}_{16-12}, \mathrm{Q}_{12-16}, \mathrm{P}_{16}$ & 8,2352 & 0,5 \\
\hline $13-15$ & 10 & 4 & $\mathbf{Q}_{15}, \mathrm{P}_{15}, \mathrm{Q}_{13-15}, \mathrm{Q}_{13}$ & 4,0613 & 0,4 \\
\hline $1-16$ & 10 & 4 & $\mathbf{Q}_{16-1}, \mathrm{P}_{16-1}, \mathrm{P}_{1-16}, \mathrm{P}_{16}$ & 15,2919 & 0,4 \\
\hline $15-45$ & 10 & 2 & $\mathbf{Q}_{15}, \mathrm{P}_{15}$ & 4,0613 & 0,2 \\
\hline
\end{tabular}




\begin{tabular}{|l|l|l|l|l|l|}
\hline $14-15$ & 10 & 2 & $\mathbf{Q}_{15}$ & 4,0613 & 0,2 \\
\hline $12-17$ & 10 & 2 & $\mathbf{Q}_{12-17}, \mathbf{Q}_{17-12}$ & 3,7579 & 0,2 \\
\hline $3-15$ & 10 & 2 & $\mathbf{Q}_{15}, \mathbf{P}_{15}$ & 4,0613 & 0,2 \\
\hline $1-15$ & 10 & 2 & $\mathbf{Q}_{15}, \mathbf{P}_{15}$ & 4,0613 & 0,2 \\
\hline $13-14$ & 10 & 1 & $\mathbf{Q}_{13}$ & 3,0831 & 0,1 \\
\hline $1-17$ & 10 & 1 & $\mathbf{Q}_{17-1}$ & 3,9165 & 0,1 \\
\hline $9-13$ & 10 & 1 & $\mathbf{Q}_{13}$ & 3,0831 & 0,1 \\
\hline $11-13$ & 10 & 1 & $\mathbf{Q}_{13}$ & 3,0831 & 0,1 \\
\hline $12-13$ & 10 & 1 & $\mathbf{Q}_{13}$ & 3,0831 & 0,1 \\
\hline $13-49$ & 10 & 1 & $\mathbf{Q}_{13}$ & 3,0831 & 0,1 \\
\hline
\end{tabular}

Tabela 5.18: Lista de ramos suspeitos utilizando somente medidas de fluxo de potência e tensão com $I S \neq 0$ - teste 5

\begin{tabular}{|l|l|l|l|l|l|}
\hline \multicolumn{1}{|c|}{ Ramo i } & \multicolumn{1}{|c|}{ TM(i) } & \multicolumn{1}{c|}{ MRE(i) } & \multicolumn{1}{c|}{ Lista de Medidas } & \multicolumn{1}{c|}{ rN } & \multicolumn{1}{c|}{ IS(i) } \\
\hline $12-16$ & 6 & 4 & $\mathbf{Q}_{16-12}, \mathrm{P}_{12-16}, \mathrm{P}_{16-12}$ & 8,9168 & 0,67 \\
\hline $1-16$ & 6 & 3 & $\mathbf{Q}_{16-1}, \mathrm{P}_{16-1}, \mathrm{P}_{1-16}$ & 15,6558 & 0,50 \\
\hline $12-17$ & 6 & 2 & $\mathbf{Q}_{12-17}, \mathrm{Q}_{17-12}$ & 5,9002 & 0,33 \\
\hline $10-12$ & 6 & 1 & $\mathbf{Q}_{12-10}$ & 3,1124 & 0,17 \\
\hline $1-17$ & 6 & 1 & $\mathbf{Q}_{17-1}$ & 6,2713 & 0,17 \\
\hline
\end{tabular}

Tabela 5.19: Parâmetros estimados do teste 5

\begin{tabular}{|c|c|c|c|c|c|}
\cline { 2 - 6 } \multicolumn{1}{c|}{} & Inicial* & Estimado & Correto & $\begin{array}{c}\text { \% de erro nos } \\
\text { valores iniciais }\end{array}$ & $\begin{array}{c}\text { \% de erro nos } \\
\text { valores estimados }\end{array}$ \\
\hline $\mathrm{G}_{12-16}$ & 1,9969 & 2,5735 & 2,5960 & 23,0 & 0,8667 \\
\hline $\mathrm{B}_{12-16}$ & $-9,0195$ & $-11,6776$ & $-11,7254$ & 23,0 & 0,4076 \\
\hline$B_{12-16}^{s h}$ & 0,0083 & 0,0109 & 0,0108 & 23,0 & 0,9259 \\
\hline
\end{tabular}

Tabela 5.20 Comparação de estimativas - Teste 5

\begin{tabular}{|c|c|c|c|c|c|c|c|}
\hline & $\begin{array}{c}\text { Banco } \\
\text { de } \\
\text { Dados } \\
\text { Inicial }\end{array}$ & $\begin{array}{c}\text { Banco de } \\
\text { Dados } \\
\text { Atualizado }\end{array}$ & $\begin{array}{l}\text { Valores } \\
\text { corretos }\end{array}$ & & $\begin{array}{c}\text { Banco de } \\
\text { Dados } \\
\text { Inicial }\end{array}$ & $\begin{array}{c}\text { Banco de } \\
\text { Dados } \\
\text { Atualizado }\end{array}$ & $\begin{array}{l}\text { Valores } \\
\text { corretos }\end{array}$ \\
\hline$\theta 1(\mathbf{r a d})$ & 0,0000 & 0,0000 & 0,0000 & V1 (pu) & 1,0391 & 1,0399 & 1,0399 \\
\hline$\theta 2$ (rad) & $-0,0212$ & $-0,0206$ & $-0,0206$ & V2 (pu) & 1,0094 & 1,0100 & 1,0101 \\
\hline$\theta 3(\mathbf{r a d})$ & $-0,1060$ & $-0,1046$ & $-0,1046$ & $\mathbf{V 3}(\mathbf{p u})$ & 0,9842 & 0,9850 & 0,9850 \\
\hline$\theta 4$ (rad) & $-0,1297$ & $-0,1282$ & $-0,1281$ & V4 (pu) & 0,9799 & 0,9808 & 0,9808 \\
\hline$\theta 5(\mathbf{r a d})$ & $-0,1508$ & $-0,1492$ & $-0,1492$ & V5 (pu) & 0,9757 & 0,9765 & 0,9765 \\
\hline$\theta 6$ (rad) & $-0,1531$ & $-0,1515$ & $-0,1514$ & V6 (pu) & 0,9792 & 0,9800 & 0,9800 \\
\hline$\theta 7(\mathbf{r a d})$ & $-0,1344$ & $-0,1328$ & $-0,1327$ & V7 (pu) & 0,9834 & 0,9842 & 0,9842 \\
\hline$\theta 8(\mathrm{rad})$ & $-0,0799$ & $-0,0783$ & $-0,0783$ & V8 (pu) & 1,0042 & 1,0050 & 1,0050 \\
\hline$\theta 9(\mathbf{r a d})$ & $-0,1691$ & $-0,1673$ & $-0,1673$ & V9 (pu) & 0,9792 & 0,9800 & 0,9801 \\
\hline$\theta 10(\mathrm{rad})$ & $-0,2017$ & $-0,1998$ & $-0,1998$ & V10 (pu) & 0,9856 & 0,9863 & 0,9863 \\
\hline$\theta 11(\mathrm{rad})$ & $-0,1798$ & $-0,1779$ & $-0,1779$ & V11(pu) & 0,9731 & 0,9740 & 0,9740 \\
\hline$\theta 12(\mathrm{rad})$ & $-0,1847$ & $-0,1828$ & $-0,1827$ & V12(pu) & 1,0145 & 1,0150 & 1,0150 \\
\hline
\end{tabular}




\begin{tabular}{|c|c|c|c|c|c|c|c|}
\hline$\theta 13$ (rad) & $-0,1730$ & $-0,1711$ & $-0,1711$ & V13(pu) & 0,9781 & 0,9789 & 0,9789 \\
\hline$\theta 14(\mathrm{rad})$ & $-0,1650$ & $-0,1632$ & $-0,1632$ & V14(pu) & 0,9693 & 0,9702 & 0,9702 \\
\hline$\theta 15(\mathrm{rad})$ & $-0,1272$ & $-0,1256$ & $-0,1256$ & V15 (pu) & 0,9872 & 0,9880 & 0,9881 \\
\hline$\theta 16$ (rad) & $-0,1493$ & $-0,1545$ & $-0,1546$ & V16 (pu) & 1,0128 & 1,0133 & 1,0134 \\
\hline$\theta 17$ (rad) & $-0,0949$ & $-0,0940$ & $-0,0940$ & V17 (pu) & 1,0166 & 1,0174 & 1,0174 \\
\hline$\theta 18$ (rad) & $-0,2064$ & $-0,2047$ & $-0,2047$ & V18 (pu) & 1,0003 & 1,0011 & 1,0011 \\
\hline$\theta 19$ (rad) & $-0,2323$ & $-0,2305$ & $-0,2305$ & V19 (pu) & 0,9696 & 0,9705 & 0,9706 \\
\hline$\theta 20$ (rad) & $-0,2361$ & $-0,2343$ & $-0,2342$ & V20(pu) & 0,9633 & 0,9642 & 0,9642 \\
\hline$\theta 21(\mathrm{rad})$ & $-0,2274$ & $-0,2255$ & $-0,2255$ & V21 (pu) & 1,0078 & 1,0087 & 1,0087 \\
\hline$\theta 22(\mathrm{rad})$ & $-0,2264$ & $-0,2245$ & $-0,2245$ & V22 (pu) & 1,0090 & 1,0099 & 1,0099 \\
\hline$\theta 23$ (rad) & $-0,2276$ & $-0,2257$ & $-0,2256$ & V23 (pu) & 1,0076 & 1,0085 & 1,0085 \\
\hline$\theta 24(\mathrm{rad})$ & $-0,2337$ & $-0,2318$ & $-0,2318$ & V24 (pu) & 0,9984 & 0,9994 & 0,9994 \\
\hline$\theta 25$ (rad) & $-0,3193$ & $-0,3172$ & $-0,3172$ & V25(pu) & 0,9816 & 0,9825 & 0,9826 \\
\hline$\theta 26(\mathrm{rad})$ & $-0,2282$ & $-0,2264$ & $-0,2263$ & $\mathrm{~V} 26(\mathrm{pu})$ & 0,9581 & 0,9590 & 0,9590 \\
\hline$\theta 27(\mathrm{rad})$ & $-0,2030$ & $-0,2012$ & $-0,2012$ & V27(pu) & 0,9806 & 0,9815 & 0,9815 \\
\hline$\theta 28(\mathrm{rad})$ & $-0,1848$ & $-0,1831$ & $-0,1830$ & V28(pu) & 0,9958 & 0,9967 & 0,9967 \\
\hline$\theta 29(\mathrm{rad})$ & $-0,1723$ & $-0,1706$ & $-0,1705$ & V29 (pu) & 1,0094 & 1,0102 & 1,0103 \\
\hline$\theta 30$ (rad) & $-0,3288$ & $-0,3267$ & $-0,3267$ & V30 (pu) & 0,9617 & 0,9627 & 0,9627 \\
\hline$\theta 31$ (rad) & $-0,3404$ & $-0,3383$ & $-0,3383$ & V31 (pu) & 0,9350 & 0,9360 & 0,9360 \\
\hline$\theta 32(\mathrm{rad})$ & $-0,3249$ & $-0,3228$ & $-0,3228$ & V32 (pu) & 0,9491 & 0,9501 & 0,9501 \\
\hline$\theta 33$ (rad) & $-0,3256$ & $-0,3235$ & $-0,3235$ & V33 (pu) & 0,9468 & 0,9478 & 0,9478 \\
\hline$\theta 34(\mathrm{rad})$ & $-0,2485$ & $-0,2465$ & $-0,2465$ & V34 (pu) & 0,9585 & 0,9595 & 0,9595 \\
\hline$\theta 35(\mathrm{rad})$ & $-0,2442$ & $-0,2423$ & $-0,2423$ & V35 (pu) & 0,9655 & 0,9665 & 0,9665 \\
\hline$\theta 36$ (rad) & $-0,2394$ & $-0,2375$ & $-0,2374$ & V36 (pu) & 0,9751 & 0,9761 & 0,9761 \\
\hline$\theta 37$ (rad) & $-0,2361$ & $-0,2342$ & $-0,2342$ & V37 (pu) & 0,9842 & 0,9851 & 0,9852 \\
\hline$\theta 38$ (rad) & $-0,2240$ & $-0,2221$ & $-0,2221$ & V38 (pu) & 1,0120 & 1,0130 & 1,0130 \\
\hline$\theta 39$ (rad) & $-0,2370$ & $-0,2350$ & $-0,2350$ & V39(pu) & 0,9822 & 0,9831 & 0,9831 \\
\hline$\theta 40(\mathrm{rad})$ & $-0,2398$ & $-0,2379$ & $-0,2378$ & $\mathrm{~V} 40(\mathrm{pu})$ & 0,9721 & 0,9730 & 0,9730 \\
\hline$\theta 41(\mathrm{rad})$ & $-0,2477$ & $-0,2457$ & $-0,2457$ & V41(pu) & 0,9954 & 0,9963 & 0,9963 \\
\hline$\theta 42(\mathrm{rad})$ & $-0,2731$ & $-0,2711$ & $-0,2710$ & $\mathrm{V42}(\mathrm{pu})$ & 0,9657 & 0,9666 & 0,9667 \\
\hline$\theta 43$ (rad) & $-0,2002$ & $-0,1983$ & $-0,1983$ & V43(pu) & 1,0087 & 1,0096 & 1,0096 \\
\hline$\theta 44$ (rad) & $-0,2086$ & $-0,2068$ & $-0,2068$ & V44(pu) & 1,0160 & 1,0169 & 1,0170 \\
\hline$\theta 45(\mathrm{rad})$ & $-0,1635$ & $-0,1618$ & $-0,1618$ & V45(pu) & 1,0352 & 1,0360 & 1,0361 \\
\hline$\theta 46(\mathrm{rad})$ & $-0,1958$ & $-0,1940$ & $-0,1939$ & V46(pu) & 1,0589 & 1,0598 & 1,0598 \\
\hline$\theta 47(\mathrm{rad})$ & $-0,2201$ & $-0,2182$ & $-0,2182$ & V47(pu) & 1,0324 & 1,0333 & 1,0333 \\
\hline$\theta 48(\mathrm{rad})$ & $-0,2219$ & $-0,2200$ & $-0,2199$ & V48(pu) & 1,0265 & 1,0274 & 1,0274 \\
\hline$\theta 49$ (rad) & $-0,2275$ & $-0,2256$ & $-0,2256$ & V49(pu) & 1,0355 & 1,0364 & 1,0364 \\
\hline$\theta 50($ rad $)$ & $-0,2359$ & $-0,2340$ & $-0,2340$ & V50 (pu) & 1,0226 & 1,0235 & 1,0235 \\
\hline$\theta 51$ (rad) & $-0,2206$ & $-0,2187$ & $-0,2186$ & V51(pu) & 1,0515 & 1,0523 & 1,0524 \\
\hline$\theta 52(\mathrm{rad})$ & $-0,2025$ & $-0,2006$ & $-0,2006$ & V52 (pu) & 0,9794 & 0,9803 & 0,9803 \\
\hline$\theta 53$ (rad) & $-0,2156$ & $-0,2138$ & $-0,2137$ & V53(pu) & 0,9700 & 0,9709 & 0,9709 \\
\hline$\theta 54(\mathrm{rad})$ & $-0,2062$ & $-0,2043$ & $-0,2043$ & V54(pu) & 0,9954 & 0,9963 & 0,9963 \\
\hline$\theta 55(\mathrm{rad})$ & $-0,1904$ & $-0,1886$ & $-0,1885$ & V55(pu) & 1,0299 & 1,0308 & 1,0308 \\
\hline$\theta 56(\mathrm{rad})$ & $-0,2825$ & $-0,2805$ & $-0,2804$ & V56(pu) & 0,9675 & 0,9685 & 0,9685 \\
\hline$\theta 57(\mathrm{rad})$ & $-0,2917$ & $-0,2897$ & $-0,2896$ & V57(pu) & 0,9641 & 0,9650 & 0,9650 \\
\hline
\end{tabular}




\section{Teste 6: Erros nos parâmetros de 2 ramos não adjacentes}

Neste caso são simulados erros simultâneos nos parâmetros de ramos não adjacentes (ramos 4-5 e 9-10). Os resultados obtidos estão expostos nas tabelas a seguir.

Tabela 5.21: Lista 1 de ramos suspeitos com $I S \neq 0$ (teste 6)

\begin{tabular}{|l|l|l|l|l|l|}
\hline \multicolumn{1}{|c|}{ Ramo i } & \multicolumn{1}{|c|}{ TM(i) } & \multicolumn{1}{c|}{ MRE(i) } & \multicolumn{1}{c|}{ Lista de Medidas } & \multicolumn{1}{c|}{$\mathbf{r}^{\mathbf{N}}$} & \multicolumn{1}{c|}{ IS(i) } \\
\hline $9-10$ & 6 & 4 & $\mathbf{P}_{10-9}, \mathrm{P}_{9-10}, \mathrm{Q}_{9-10}, \mathrm{Q}_{10-9}$ & 23,69 & 0,67 \\
\hline $5-6$ & 6 & 4 & $\mathbf{P}_{5-6}, \mathrm{P}_{6-5}, \mathrm{Q}_{5-6}, \mathrm{Q}_{6-5}$ & 4,84 & 0,67 \\
\hline $10-51$ & 6 & 4 & $\mathbf{P}_{10-51}, \mathrm{P}_{51-10}, \mathrm{Q}_{51-10}, \mathrm{Q}_{10-51}$ & 6,92 & 0,67 \\
\hline $10-12$ & 6 & 4 & $\mathbf{P}_{12-10}, \mathrm{P}_{10-12}, \mathrm{Q}_{12-10}, \mathrm{Q}_{10-12}$ & 19,38 & 0,67 \\
\hline $4-5$ & 6 & 3 & $\mathbf{P}_{5-4}, \mathrm{P}_{4-5}, \mathrm{Q}_{4-5}$ & 13,55 & 0,50 \\
\hline $4-6$ & 6 & 3 & $\mathbf{P}_{6-4}, \mathrm{P}_{4-6}, \mathrm{Q}_{6-4}$ & 15,93 & 0,50 \\
\hline $49-50$ & 6 & 2 & $\mathbf{Q}_{49-50}, \mathrm{Q}_{50-49}$ & 6,01 & 0,33 \\
\hline $9-13$ & 6 & 2 & $\mathbf{P}_{9-13}, \mathrm{P}_{13-9}$, & 6,31 & 0,33 \\
\hline $50-51$ & 6 & 2 & $\mathbf{Q}_{50-51}, \mathrm{Q}_{51-50}$ & 8,54 & 0,33 \\
\hline $8-9$ & 6 & 2 & $\mathbf{Q}_{9-8}, \mathrm{Q}_{8-9}$ & 6,32 & 0,33 \\
\hline $15-45$ & 6 & 2 & $\mathbf{P}_{45-15}, \mathrm{P}_{15-45}$ & 3,81 & 0,33 \\
\hline $9-11$ & 6 & 2 & $\mathbf{P}_{11-9}, \mathrm{P}_{9-11}$ & 4,97 & 0,33 \\
\hline $9-12$ & 6 & 2 & $\mathbf{Q}_{12-9}, \mathrm{P}_{9-12}$ & 4,76 & 0,33 \\
\hline $48-49$ & 6 & 2 & $\mathbf{Q}_{48-49}, \mathrm{Q}_{49-48}$ & 4,81 & 0,33 \\
\hline $11-13$ & 6 & 1 & $\mathbf{P}_{11-13}$ & 5,69 & 0,17 \\
\hline $12-13$ & 6 & 1 & $\mathbf{P}_{13-12}$ & 5,43 & 0,17 \\
\hline $1-2$ & 6 & 1 & $\mathbf{Q}_{2-1}$ & 3,40 & 0,17 \\
\hline $3-4$ & 6 & 1 & $\mathbf{P}_{4-3}$ & 3,14 & 0,17 \\
\hline
\end{tabular}

Tabela 5.22: Parâmetros estimados do teste 6

\begin{tabular}{|c|c|c|c|c|c|}
\cline { 2 - 6 } \multicolumn{1}{c|}{} & Inicial* & Estimado & Correto & $\begin{array}{c}\text { \% de erro nos valores } \\
\text { iniciais }\end{array}$ & $\begin{array}{c}\text { \% de erro nos valores } \\
\text { estimados }\end{array}$ \\
\hline $\mathrm{G}_{45}$ & 3,8091 & 2,9519 & 2,9301 & 30,0 & 0,7440 \\
\hline $\mathrm{B}_{45}$ & $-8,0449$ & $-6,1923$ & $-6,1884$ & 30,0 & 0,0630 \\
\hline$B_{4-5}^{\text {shunt }}$ & 0,0168 & 0,0130 & 0,0129 & 30,0 & 0,7751 \\
\hline $\mathrm{G}_{9-10}$ & 1,6232 & 1,2225 & 1,2486 & 30,0 & 2,0903 \\
\hline $\mathrm{B}_{9-10}$ & 7,3859 & $-5,6449$ & $-5,6815$ & 30,0 & 0,6441 \\
\hline$B_{9-10}^{\text {shunt }}$ & 0,0286 & 0,0224 & 0,0220 & 30,0 & 1,8181 \\
\hline
\end{tabular}

Tabela 5.23 Comparação de estimativas - Teste 6

\begin{tabular}{|c|c|c|c|c|c|c|c|}
\hline & $\begin{array}{c}\text { Banco } \\
\text { de } \\
\text { Dados } \\
\text { Inicial }\end{array}$ & $\begin{array}{c}\text { Banco de } \\
\text { Dados } \\
\text { Atualizado }\end{array}$ & $\begin{array}{c}\text { Valores } \\
\text { corretos }\end{array}$ & $\begin{array}{c}\text { Banco de } \\
\text { Dados } \\
\text { Inicial }\end{array}$ & $\begin{array}{c}\text { Banco de } \\
\text { Dados } \\
\text { Atualizado }\end{array}$ & $\begin{array}{c}\text { Valores } \\
\text { corretos }\end{array}$ \\
\hline $\boldsymbol{\theta 1}(\mathbf{r a d})$ & 0,0000 & 0,0000 & 0,0000 & $\mathbf{V 1}(\mathbf{p u})$ & 1,0389 & 1,0399 & 1,0399 \\
\hline $\boldsymbol{\theta 2}(\mathbf{r a d})$ & $-0,0209$ & $-0,0206$ & $-0,0206$ & $\mathbf{V 2}(\mathbf{p u})$ & 1,0094 & 1,0100 & 1,0101 \\
\hline
\end{tabular}


104

\begin{tabular}{|c|c|c|c|c|c|c|c|}
\hline$\theta 3$ (rad) & $-0,1051$ & $-0,1046$ & $-0,1046$ & V3 (pu) & 0,9844 & 0,9850 & 0,9850 \\
\hline$\theta 4(\mathrm{rad})$ & $-0,1293$ & $-0,1281$ & $-0,1281$ & V4 (pu) & 0,9800 & 0,9807 & 0,9808 \\
\hline$\theta 5$ (rad) & $-0,1472$ & $-0,1492$ & $-0,1492$ & V5 (pu) & 0,9762 & 0,9764 & 0,9765 \\
\hline$\theta 6(\mathrm{rad})$ & $-0,1497$ & $-0,1514$ & $-0,1514$ & V6 (pu) & 0,9798 & 0,9799 & 0,9800 \\
\hline$\theta 7(\mathrm{rad})$ & $-0,1311$ & $-0,1327$ & $-0,1327$ & V7 (pu) & 0,9840 & 0,9841 & 0,9842 \\
\hline$\theta 8(\mathbf{r a d})$ & $-0,0776$ & $-0,0782$ & $-0,0783$ & V8 (pu) & 1,0047 & 1,0049 & 1,0050 \\
\hline$\theta 9$ (rad) & $-0,1679$ & $-0,1673$ & $-0,1673$ & V9 (pu) & 0,9795 & 0,9800 & 0,9801 \\
\hline$\theta 10(\mathrm{rad})$ & $-0,1966$ & $-0,1998$ & $-0,1998$ & V10 (pu) & 0,9841 & 0,9864 & 0,9863 \\
\hline$\theta 11(\mathrm{rad})$ & $-0,1780$ & $-0,1779$ & $-0,1779$ & V11(pu) & 0,9735 & 0,9739 & 0,9740 \\
\hline$\theta 12(\mathrm{rad})$ & $-0,1824$ & $-0,1827$ & $-0,1827$ & V12(pu) & 1,0138 & 1,0149 & 1,0150 \\
\hline$\theta 13(\mathrm{rad})$ & $-0,1711$ & $-0,1711$ & $-0,1711$ & V13(pu) & 0,9785 & 0,9789 & 0,9789 \\
\hline$\theta 14(\mathrm{rad})$ & $-0,1632$ & $-0,1632$ & $-0,1632$ & V14(pu) & 0,9697 & 0,9702 & 0,9702 \\
\hline$\theta 15(\mathrm{rad})$ & $-0,1258$ & $-0,1256$ & $-0,1256$ & V15 (pu) & 0,9874 & 0,9880 & 0,9881 \\
\hline$\theta 16$ (rad) & $-0,1543$ & $-0,1546$ & $-0,1546$ & V16 (pu) & 1,0123 & 1,0133 & 1,0134 \\
\hline$\theta 17$ (rad) & $-0,0938$ & $-0,0940$ & $-0,0940$ & V17 (pu) & 1,0164 & 1,0174 & 1,0174 \\
\hline$\theta 18(\mathrm{rad})$ & $-0,2051$ & $-0,2047$ & $-0,2047$ & V18 (pu) & 1,0003 & 1,0011 & 1,0011 \\
\hline$\theta 19$ (rad) & $-0,2305$ & $-0,2305$ & $-0,2305$ & V19 (pu) & 0,9697 & 0,9705 & 0,9706 \\
\hline$\theta 20$ (rad) & $-0,2341$ & $-0,2343$ & $-0,2342$ & V20(pu) & 0,9633 & 0,9642 & 0,9642 \\
\hline$\theta 21(\mathrm{rad})$ & $\begin{array}{l}-0,2248 \\
\end{array}$ & $-0,2255$ & $-0,2255$ & V21 (pu) & 1,0078 & 1,0087 & 1,0087 \\
\hline$\theta 22(\mathrm{rad})$ & $-0,2239$ & $-0,2245$ & $-0,2245$ & V22 (pu) & 1,0090 & 1,0099 & 1,0099 \\
\hline$\theta 23(\mathrm{rad})$ & $-0,2250$ & $-0,2257$ & $\begin{array}{l}-0,2256 \\
\end{array}$ & V23 (pu) & 1,0076 & 1,0085 & 1,0085 \\
\hline$\theta 24(\mathrm{rad})$ & $-0,2312$ & $-0,2318$ & $-0,2318$ & V24 (pu) & 0,9986 & 0,9993 & 0,9994 \\
\hline$\theta 25(\mathrm{rad})$ & $-0,3168$ & $-0,3172$ & $-0,3172$ & V25(pu) & 0,9818 & 0,9825 & 0,9826 \\
\hline$\theta 26(\mathrm{rad})$ & $-0,2258$ & $-0,2263$ & $-0,2263$ & V26(pu) & 0,9583 & 0,9589 & 0,9590 \\
\hline$\theta 27(\mathrm{rad})$ & $-0,2006$ & $-0,2012$ & $-0,2012$ & V27(pu) & 0,9811 & 0,9814 & 0,9815 \\
\hline$\theta 28(\mathrm{rad})$ & $-0,1823$ & $-0,1830$ & $-0,1830$ & V28(pu) & 0,9964 & 0,9966 & 0,9967 \\
\hline$\theta 29(\mathrm{rad})$ & $-0,1697$ & $-0,1705$ & $-0,1705$ & V29 (pu) & 1,0100 & 1,0102 & 1,0103 \\
\hline$\theta 30$ (rad) & $-0,3263$ & $-0,3267$ & $-0,3267$ & V30 (pu) & 0,9619 & 0,9626 & 0,9627 \\
\hline$\theta 31$ (rad) & $-0,3379$ & $-0,3383$ & $-0,3383$ & V31 (pu) & 0,9351 & 0,9359 & 0,9360 \\
\hline$\theta 32(\mathrm{rad})$ & $-0,3224$ & $-0,3228$ & $-0,3228$ & V32 (pu) & 0,9491 & 0,9500 & 0,9501 \\
\hline$\theta 33(\mathrm{rad})$ & $-0,3231$ & $-0,3235$ & $-0,3235$ & V33 (pu) & 0,9469 & 0,9477 & 0,9478 \\
\hline$\theta 34(\mathrm{rad})$ & $-0,2460$ & $-0,2465$ & $-0,2465$ & V34 (pu) & 0,9585 & 0,9594 & 0,9595 \\
\hline$\theta 35($ rad $)$ & $-0,2417$ & $-0,2423$ & $-0,2423$ & V35 (pu) & 0,9655 & 0,9664 & 0,9665 \\
\hline$\theta 36$ (rad) & $-0,2369$ & $-0,2375$ & $-0,2374$ & V36 (pu) & 0,9752 & 0,9760 & 0,9761 \\
\hline$\theta 37$ (rad) & $-0,2336$ & $-0,2342$ & $-0,2342$ & V37 (pu) & 0,9842 & 0,9851 & 0,9852 \\
\hline$\theta 38(\mathrm{rad})$ & $-0,2215$ & $-0,2221$ & $-0,2221$ & V38 (pu) & 1,0121 & 1,0129 & 1,0130 \\
\hline$\theta 39(\mathrm{rad})$ & $-0,2345$ & $-0,2350$ & $-0,2350$ & V39(pu) & 0,9822 & 0,9831 & 0,9831 \\
\hline$\theta 40(\mathrm{rad})$ & $-0,2373$ & $-0,2379$ & $-0,2378$ & V40(pu) & 0,9721 & 0,9730 & 0,9730 \\
\hline$\theta 41(\mathrm{rad})$ & $-0,2458$ & $-0,2457$ & $-0,2457$ & V41(pu) & 0,9958 & 0,9963 & 0,9963 \\
\hline$\theta 42(\mathrm{rad})$ & $-0,2711$ & $-0,2710$ & $-0,2710$ & V42(pu) & 0,9660 & 0,9666 & 0,9667 \\
\hline$\theta 43(\mathrm{rad})$ & $-0,1984$ & $-0,1983$ & $-0,1983$ & V43(pu) & 1,0091 & 1,0095 & 1,0096 \\
\hline$\theta 44$ (rad) & $-0,2061$ & $-0,2068$ & $-0,2068$ & V44(pu) & 1,0161 & 1,0169 & 1,0170 \\
\hline$\theta 45(\mathrm{rad})$ & $-0,1612$ & $-0,1618$ & $-0,1618$ & V45(pu) & 1,0353 & 1,0360 & 1,0361 \\
\hline$\theta 46(\mathrm{rad})$ & $-0,1936$ & $-0,1939$ & $-0,1939$ & V46(pu) & 1,0591 & 1,0598 & 1,0598 \\
\hline$\theta 47(\mathrm{rad})$ & $-0,2175$ & $-0,2182$ & $-0,2182$ & V47(pu) & 1,0323 & 1,0333 & 1,0333 \\
\hline$\theta 48(\mathrm{rad})$ & $-0,2192$ & $-0,2200$ & $-0,2199$ & V48(pu) & 1,0264 & 1,0274 & 1,0274 \\
\hline$\theta 49(\mathrm{rad})$ & $-0,2247$ & $-0,2256$ & $-0,2256$ & V49(pu) & 1,0351 & 1,0363 & 1,0364 \\
\hline$\theta 50(\mathrm{rad})$ & $-0,2329$ & $-0,2340$ & $-0,2340$ & V50 (pu) & 1,0219 & 1,0235 & 1,0235 \\
\hline
\end{tabular}




\begin{tabular}{|c|c|c|c|c|c|c|c|}
\hline$\theta 51(\mathrm{rad})$ & $-0,2166$ & $-0,2187$ & $-0,2186$ & V51(pu) & 1,0502 & 1,0524 & 1,0524 \\
\hline$\theta 52(\mathrm{rad})$ & $\begin{array}{l}-0,2002 \\
\end{array}$ & $-0,2006$ & $-0,2006$ & V52 (pu) & 0,9799 & 0,9803 & 0,9803 \\
\hline$\theta 53(\mathrm{rad})$ & $-0,2135$ & $-0,2137$ & $-0,2137$ & V53(pu) & 0,9705 & 0,9708 & 0,9709 \\
\hline$\theta 54(\mathrm{rad})$ & $\begin{array}{l}-0,2043 \\
\end{array}$ & $-0,2043$ & $\begin{array}{l}-0,2043 \\
\end{array}$ & V54(pu) & 0,9959 & 0,9962 & 0,9963 \\
\hline$\theta 55(\mathrm{rad})$ & $\begin{array}{l}-0,1890 \\
\end{array}$ & $-0,1885$ & $-0,1885$ & V55(pu) & 1,0302 & 1,0307 & 1,0308 \\
\hline$\theta 56(\mathrm{rad})$ & $-0,2804$ & $-0,2805$ & $-0,2804$ & V56(pu) & 0,9679 & 0,9684 & 0,9685 \\
\hline$\theta 57(\mathrm{rad})$ & $\begin{array}{l}-0,2896 \\
\end{array}$ & $-0,2897$ & $-0,2896$ & V57(pu) & 0,9643 & 0,9650 & 0,9650 \\
\hline
\end{tabular}

\section{Teste 7: Erros nos parâmetros de 3 ramos}

No Teste 6 foram simulados erros simultâneos em ramos não adjacentes. Neste teste são simulados erros em parâmetros de três ramos (ramos 7-8, 1-15 e 3-15), sendo dois adjacentes (ramos 1-15 e 3-15). Os resultados obtidos estão expostos nas tabelas a seguir.

Tabela 5.24: Lista 1 de ramos suspeitos com $I S \neq 0$ (teste 7)

\begin{tabular}{|l|l|l|l|l|l|}
\hline \multicolumn{1}{|c|}{ Ramo i } & \multicolumn{1}{|c|}{ TM(i) } & \multicolumn{1}{c|}{ MRE(i) } & \multicolumn{1}{|c|}{ Lista de Medidas } & \multicolumn{1}{c|}{$\mathbf{r}^{\mathbf{N}}$} & IS(i) \\
\hline $8-9$ & 6 & 4 & $\mathbf{Q}_{9-8}, \mathrm{Q}_{8-9}, \mathrm{P}_{8-9}, \mathrm{P}_{9-8}$ & 13,48 & 0,67 \\
\hline $1-2$ & 6 & 4 & $\mathbf{Q}_{2-1}, \mathrm{Q}_{1-2}, \mathrm{P}_{2-1}, \mathrm{P}_{1-2}$ & 11,77 & 0,67 \\
\hline $1-15$ & 6 & 4 & $\mathbf{P}_{15-1}, \mathrm{P}_{1-15}, \mathrm{Q}_{15-1}, \mathrm{Q}_{1-15}$ & 26,57 & 0,67 \\
\hline $7-8$ & 6 & 4 & $\mathbf{P}_{8-7}, \mathrm{P}_{7-8}, \mathrm{Q}_{7-8}, \mathrm{Q}_{8-7}$ & 20,40 & 0,67 \\
\hline $6-8$ & 6 & 4 & $\mathbf{Q}_{8-6}, \mathrm{Q}_{6-8}, \mathrm{P}_{6-8}, \mathrm{P}_{8-6}$ & 17,34 & 0,67 \\
\hline $6-7$ & 6 & 4 & $\mathbf{P}_{7-6}, \mathrm{P}_{6-7}, \mathrm{Q}_{6-7}, \mathrm{Q}_{7-6}$ & & 0,67 \\
\hline $1-17$ & 6 & 4 & $\mathbf{P}_{1-17}, \mathrm{Q}_{1-17}, \mathrm{P}_{17-1}, \mathrm{Q}_{17-1}$ & 7,11 & 0,67 \\
\hline $1-16$ & 6 & 4 & $\mathbf{Q}_{1-16}, \mathrm{P}_{16-1}, \mathrm{P}_{1-16}, \mathrm{Q}_{16-1}$ & 9,61 & 0,67 \\
\hline $13-15$ & 6 & 3 & $\mathbf{P}_{15-13}, \mathrm{P}_{13-15}, \mathrm{Q}_{13-15}$ & 8,32 & 0,50 \\
\hline $12-17$ & 6 & 3 & $\mathbf{P}_{12-17}, \mathrm{P}_{17-12}, \mathrm{Q}_{17-12}$ & 7,80 & 0,50 \\
\hline $12-16$ & 6 & 3 & $\mathbf{P}_{12-16}, \mathrm{Q}_{12-16}, \mathrm{P}_{16-12}$ & 4,05 & 0,50 \\
\hline $2-3$ & 6 & 3 & $\mathbf{Q}_{2-3}, \mathrm{P}_{2-3}, \mathrm{P}_{3-2}$ & 11,75 & 0,50 \\
\hline $14-15$ & 6 & 2 & $\mathbf{P}_{14-15}, \mathrm{P}_{15-14}$ & 5,40 & 0,33 \\
\hline $3-4$ & 6 & 2 & $\mathbf{P}_{3-4}, \mathrm{P}_{4-3}$ & 7,43 & 0,33 \\
\hline $9-12$ & 6 & 1 & $\mathbf{Q}_{12-9}$ & & 0,17 \\
\hline $4-6$ & 6 & 1 & $\mathbf{Q}_{6-4}$, & & 0,17 \\
\hline $44-45$ & 6 & 1 & $\mathbf{P}_{44-45}$ & 3,01 & 0,17 \\
\hline $3-15$ & 6 & 1 & $\mathbf{Q}_{3-15}$, & 15,22 & 0,17 \\
\hline $10-12$ & 6 & 1 & $\mathbf{Q}_{12-10}$ & 3,53 & 0,17 \\
\hline $54-55$ & 6 & 1 & $\mathbf{P}_{54-55}$ & 3,43 & 0,50 \\
\hline
\end{tabular}

Tabela 5.25: Parâmetros estimados do teste 7

\begin{tabular}{|c|c|c|c|c|c|}
\cline { 2 - 6 } \multicolumn{1}{c|}{} & Inicial* & Estimado & Corretos & $\begin{array}{c}\text { \% de erro nos valores } \\
\text { iniciais }\end{array}$ & $\begin{array}{c}\text { \% de erro nos valores } \\
\text { estimados }\end{array}$ \\
\hline $\mathrm{G}_{7-8}$ & 3,4337 & 2,6428 & 2,6413 & 30,0 & 0,0567 \\
\hline $\mathrm{B}_{7-8}$ & $-17,5881$ & $-13,4449$ & $-13,5293$ & 30,0 & 0,6238 \\
\hline$B_{7-8}^{\text {shunt }}$ & 0,0126 & 0,0093 & 0,0097 & 30,0 & 4,1237 \\
\hline
\end{tabular}




\begin{tabular}{|c|c|c|c|c|c|}
\hline $\mathrm{G}_{1-15}$ & 2,6914 & 2,0750 & 2,0703 & 30,0 & 0,2270 \\
\hline $\mathrm{B}_{1-15}$ & $-13,7593$ & $-10,5946$ & $-10,5841$ & 30,0 & 0,0992 \\
\hline$B_{1-15}^{\text {shunt }}$ & 0,0642 & 0,0490 & 0,0494 & 30,0 & 0,8097 \\
\hline $\mathrm{G}_{3-15}$ & 6,8567 & 5,1011 & 5,2744 & 30,0 & 3,2856 \\
\hline $\mathrm{B}_{3-15}$ & 22,4225 & $-17,4270$ & $-17,2481$ & 30,0 & 1,0372 \\
\hline$B_{3-15}^{\text {shunt }}$ & 0,0354 & 0,0276 & 0,0272 & 30,0 & 1,4702 \\
\hline
\end{tabular}

Tabela 5.26 Comparação de estimativas - Teste 7

\begin{tabular}{|c|c|c|c|c|c|c|c|}
\hline & $\begin{array}{c}\text { Banco de } \\
\text { Dados } \\
\text { Inicial } \\
\end{array}$ & $\begin{array}{c}\text { Banco de } \\
\text { Dados } \\
\text { Atualizado } \\
\end{array}$ & $\begin{array}{l}\text { Valores } \\
\text { corretos }\end{array}$ & & $\begin{array}{c}\text { Banco de } \\
\text { Dados } \\
\text { Inicial } \\
\end{array}$ & $\begin{array}{c}\text { Banco de } \\
\text { Dados } \\
\text { Atualizado } \\
\end{array}$ & $\begin{array}{l}\text { Valores } \\
\text { corretos }\end{array}$ \\
\hline$\theta 1(\operatorname{rad})$ & 0,0000 & 0,0000 & 0,0000 & V1 (pu) & 1,0310 & 1,0400 & 1,0399 \\
\hline$\theta 2(\mathrm{rad})$ & $-0,0200$ & $-0,0206$ & $-0,0206$ & V2 (pu) & 1,0032 & 1,0100 & 1,0101 \\
\hline$\theta 3(\mathrm{rad})$ & $-0,1014$ & $-0,1047$ & $-0,1046$ & V3 (pu) & 0,9794 & 0,9849 & 0,9850 \\
\hline$\theta 4$ (rad) & $-0,1238$ & $-0,1282$ & $-0,1281$ & V4 (pu) & 0,9756 & 0,9807 & 0,9808 \\
\hline$\theta 5$ (rad) & $-0,1446$ & $-0,1492$ & $-0,1492$ & V5 (pu) & 0,9712 & 0,9765 & 0,9765 \\
\hline$\theta 6(\mathrm{rad})$ & $-0,1469$ & $-0,1515$ & $-0,1514$ & V6 (pu) & 0,9747 & 0,9800 & 0,9800 \\
\hline$\theta 7(\mathrm{rad})$ & $-0,1271$ & $-0,1328$ & $-0,1327$ & V7 (pu) & 0,9794 & 0,9841 & 0,9842 \\
\hline$\theta 8$ (rad) & $-0,0781$ & $-0,0782$ & $-0,0783$ & V8 (pu) & 0,9972 & 1,0051 & 1,0050 \\
\hline$\theta 9$ (rad) & $-0,1626$ & $-0,1672$ & $-0,1673$ & V9 (pu) & 0,9734 & 0,9801 & 0,9801 \\
\hline$\theta 10(\mathrm{rad})$ & $-0,1954$ & $-0,1997$ & $-0,1998$ & V10 (pu) & 0,9797 & 0,9864 & 0,9863 \\
\hline$\theta 11(\mathrm{rad})$ & $-0,1733$ & $-0,1778$ & $-0,1779$ & V11(pu) & 0,9673 & 0,9741 & 0,9740 \\
\hline$\theta 12(\mathrm{rad})$ & $-0,1780$ & $-0,1827$ & $-0,1827$ & V12(pu) & 1,0079 & 1,0150 & 1,0150 \\
\hline$\theta 13(\mathrm{rad})$ & $-0,1663$ & $-0,1711$ & $-0,1711$ & V13(pu) & 0,9723 & 0,9790 & 0,9789 \\
\hline$\theta 14(\mathrm{rad})$ & $-0,1582$ & $-0,1632$ & $-0,1632$ & V14(pu) & 0,9635 & 0,9703 & 0,9702 \\
\hline$\theta 15(\mathrm{rad})$ & $-0,1181$ & $-0,1255$ & $-0,1256$ & V15 (pu) & 0,9820 & 0,9882 & 0,9881 \\
\hline$\theta 16$ (rad) & $-0,1502$ & $-0,1545$ & $-0,1546$ & V16 (pu) & 1,0060 & 1,0134 & 1,0134 \\
\hline$\theta 17$ (rad) & $-0,0918$ & $-0,0940$ & $-0,0940$ & V17 (pu) & 1,0093 & 1,0174 & 1,0174 \\
\hline$\theta 18$ (rad) & $-0,2007$ & $-0,2047$ & $-0,2047$ & V18 (pu) & 0,9957 & 1,0011 & 1,0011 \\
\hline$\theta 19$ (rad) & $-0,2266$ & $-0,2305$ & $-0,2305$ & V19 (pu) & 0,9645 & 0,9705 & 0,9706 \\
\hline$\theta 20$ (rad) & $-0,2303$ & $-0,2342$ & $-0,2342$ & V20(pu) & 0,9579 & 0,9642 & 0,9642 \\
\hline$\theta 21(\mathrm{rad})$ & $-0,2212$ & $-0,2254$ & $-0,2255$ & V21 (pu) & 1,0016 & 1,0088 & 1,0087 \\
\hline$\theta 22(\mathrm{rad})$ & $-0,2202$ & $-0,2245$ & $-0,2245$ & V22 (pu) & 1,0028 & 1,0100 & 1,0099 \\
\hline$\theta 23(\mathrm{rad})$ & $-0,2214$ & $-0,2256$ & $-0,2256$ & V23 (pu) & 1,0014 & 1,0086 & 1,0085 \\
\hline$\theta 24(\mathrm{rad})$ & $-0,2277$ & $-0,2317$ & $-0,2318$ & V24 (pu) & 0,9926 & 0,9994 & 0,9994 \\
\hline$\theta 25(\mathrm{rad})$ & $-0,3143$ & $-0,3171$ & $-0,3172$ & V25(pu) & 0,9756 & 0,9826 & 0,9826 \\
\hline$\theta 26(\mathrm{rad})$ & $-0,2222$ & $-0,2263$ & $-0,2263$ & V26(pu) & 0,9525 & 0,9590 & 0,9590 \\
\hline$\theta 27(\mathrm{rad})$ & $-0,1965$ & $-0,2012$ & $-0,2012$ & V27(pu) & 0,9757 & 0,9815 & 0,9815 \\
\hline$\theta 28(\mathrm{rad})$ & $-0,1777$ & $-0,1830$ & $-0,1830$ & V28(pu) & 0,9913 & 0,9966 & 0,9967 \\
\hline$\theta 29($ rad $)$ & $-0,1650$ & $-0,1706$ & $-0,1705$ & V29 (pu) & 1,0051 & 1,0102 & 1,0103 \\
\hline$\theta 30(\mathrm{rad})$ & $-0,3239$ & $-0,3266$ & $-0,3267$ & V30 (pu) & 0,9556 & 0,9627 & 0,9627 \\
\hline$\theta 31$ (rad) & $-0,3357$ & $-0,3382$ & $-0,3383$ & V31 (pu) & 0,9286 & 0,9360 & 0,9360 \\
\hline$\theta 32(\mathrm{rad})$ & $-0,3200$ & $-0,3227$ & $-0,3228$ & V32 (pu) & 0,9426 & 0,9501 & 0,9501 \\
\hline$\theta 33(\mathrm{rad})$ & $-0,3207$ & $-0,3234$ & $-0,3235$ & V33 (pu) & 0,9402 & 0,9479 & 0,9478 \\
\hline$\theta 34(\mathrm{rad})$ & $-0,2425$ & $-0,2464$ & $-0,2465$ & V34 (pu) & 0,9521 & 0,9596 & 0,9595 \\
\hline$\theta 35(\mathrm{rad})$ & $-0,2382$ & $-0,2422$ & $-0,2423$ & V35 (pu) & 0,9592 & 0,9665 & 0,9665 \\
\hline$\theta 36$ (rad) & $-0,2333$ & $-0,2374$ & $-0,2374$ & V36 (pu) & 0,9689 & 0,9762 & 0,9761 \\
\hline
\end{tabular}




\begin{tabular}{|l|l|l|l|l|l|l|l|}
\hline $\boldsymbol{\theta 3 7}(\mathbf{r a d})$ & $-0,2300$ & $-0,2341$ & $-0,2342$ & $\mathbf{V 3 7}(\mathbf{p u})$ & 0,9780 & 0,9852 & 0,9852 \\
\hline $\boldsymbol{\theta 3 8}(\mathbf{r a d})$ & $-0,2178$ & $-0,2220$ & $-0,2221$ & $\mathbf{V 3 8}(\mathbf{p u})$ & 1,0059 & 1,0131 & 1,0130 \\
\hline $\boldsymbol{\theta 3 9}(\mathbf{r a d})$ & $-0,2308$ & $-0,2350$ & $-0,2350$ & $\mathbf{V 3 9}(\mathbf{p u})$ & 0,9759 & 0,9832 & 0,9831 \\
\hline $\boldsymbol{\theta 4 0}(\mathbf{r a d})$ & $-0,2337$ & $-0,2378$ & $-0,2378$ & $\mathbf{V 4 0}(\mathbf{p u})$ & 0,9658 & 0,9731 & 0,9730 \\
\hline $\boldsymbol{\theta 4 1}(\mathbf{r a d})$ & $-0,2420$ & $-0,2456$ & $-0,2457$ & $\mathbf{V 4 1}(\mathbf{p u})$ & 0,9892 & 0,9964 & 0,9963 \\
\hline $\boldsymbol{\theta 4 2}(\mathbf{r a d})$ & $-0,2676$ & $-0,2710$ & $-0,2710$ & $\mathbf{V 4 2}(\mathbf{p u})$ & 0,9593 & 0,9667 & 0,9667 \\
\hline $\boldsymbol{\theta 4 3}(\mathbf{r a d})$ & $-0,1939$ & $-0,1982$ & $-0,1983$ & $\mathbf{V 4 3}(\mathbf{p u})$ & 1,0026 & 1,0097 & 1,0096 \\
\hline $\boldsymbol{\theta 4 4}(\mathbf{r a d})$ & $-0,2020$ & $-0,2067$ & $-0,2068$ & $\mathbf{V 4 4}(\mathbf{p u})$ & 1,0100 & 1,0170 & 1,0170 \\
\hline $\boldsymbol{\theta 4 5}(\mathbf{r a d})$ & $-0,1554$ & $-0,1617$ & $-0,1618$ & $\mathbf{V 4 5}(\mathbf{p u})$ & 1,0298 & 1,0362 & 1,0361 \\
\hline $\boldsymbol{\theta 4 6}(\mathbf{r a d})$ & $-0,1893$ & $-0,1939$ & $-0,1939$ & $\mathbf{V 4 6}(\mathbf{p u})$ & 1,0526 & 1,0599 & 1,0598 \\
\hline $\boldsymbol{\theta 4 7}(\mathbf{r a d})$ & $-0,2139$ & $-0,2182$ & $-0,2182$ & $\mathbf{V 4 7}(\mathbf{p u})$ & 1,0263 & 1,0334 & 1,0333 \\
\hline $\boldsymbol{\theta 4 8}(\mathbf{r a d})$ & $-0,2156$ & $-0,2199$ & $-0,2199$ & $\mathbf{V 4 8}(\mathbf{p u})$ & 1,0204 & 1,0275 & 1,0274 \\
\hline $\boldsymbol{\theta 4 9}(\mathbf{r a d})$ & $-0,2213$ & $-0,2255$ & $-0,2256$ & $\mathbf{V 4 9}(\mathbf{p u})$ & 1,0294 & 1,0364 & 1,0364 \\
\hline $\boldsymbol{\theta 5 0}(\mathbf{r a d})$ & $-0,2298$ & $-0,2339$ & $-0,2340$ & $\mathbf{V 5 0}(\mathbf{p u})$ & 1,0163 & 1,0235 & 1,0235 \\
\hline $\boldsymbol{\theta 5 1}(\mathbf{r a d})$ & $-0,2145$ & $-0,2186$ & $-0,2186$ & $\mathbf{V 5 1}(\mathbf{p u})$ & 1,0452 & 1,0524 & 1,0524 \\
\hline $\boldsymbol{\theta 5 2}(\mathbf{r a d})$ & $-0,1958$ & $-0,2006$ & $-0,2006$ & $\mathbf{V 5 2}(\mathbf{p u})$ & 0,9744 & 0,9803 & 0,9803 \\
\hline $\boldsymbol{\theta 5 3}(\mathbf{r a d})$ & $-0,2092$ & $-0,2137$ & $-0,2137$ & $\mathbf{V 5 3}(\mathbf{p u})$ & 0,9648 & 0,9709 & 0,9709 \\
\hline $\boldsymbol{\theta 5 4}(\mathbf{r a d})$ & $-0,1996$ & $-0,2043$ & $-0,2043$ & $\mathbf{V 5 4}(\mathbf{p u})$ & 0,9899 & 0,9963 & 0,9963 \\
\hline $\boldsymbol{\theta 5 5}(\mathbf{r a d})$ & $-0,1842$ & $-0,1885$ & $-0,1885$ & $\mathbf{V 5 5}(\mathbf{p u})$ & 1,0238 & 1,0308 & 1,0308 \\
\hline $\boldsymbol{\theta 5 6}(\mathbf{r a d})$ & $-0,2772$ & $-0,2804$ & $-0,2804$ & $\mathbf{V 5 6}(\mathbf{p u})$ & 0,9611 & 0,9686 & 0,9685 \\
\hline $\boldsymbol{\theta 5 7}(\mathbf{r a d})$ & $-0,2865$ & $-0,2896$ & $-0,2896$ & $\mathbf{V 5 7}(\mathbf{p u})$ & 0,9576 & 0,9651 & 0,9650 \\
\hline
\end{tabular}

\subsection{SIMULAÇÕES COM O SISTEMA IEEE DE 30 BARRAS}

A metodologia proposta é aplicada agora ao sistema de 30 barras do IEEE, apresentado na Figura 5.3. Em todas as 9 amostras que foram geradas para realização desses testes, considerou-se que estão disponíveis todas as medidas indicadas no sistema de medição encontrado na Figura 5.3. A Tabela 5.27 apresenta os valores corretos das condutâncias e susceptâncias do sistema. 


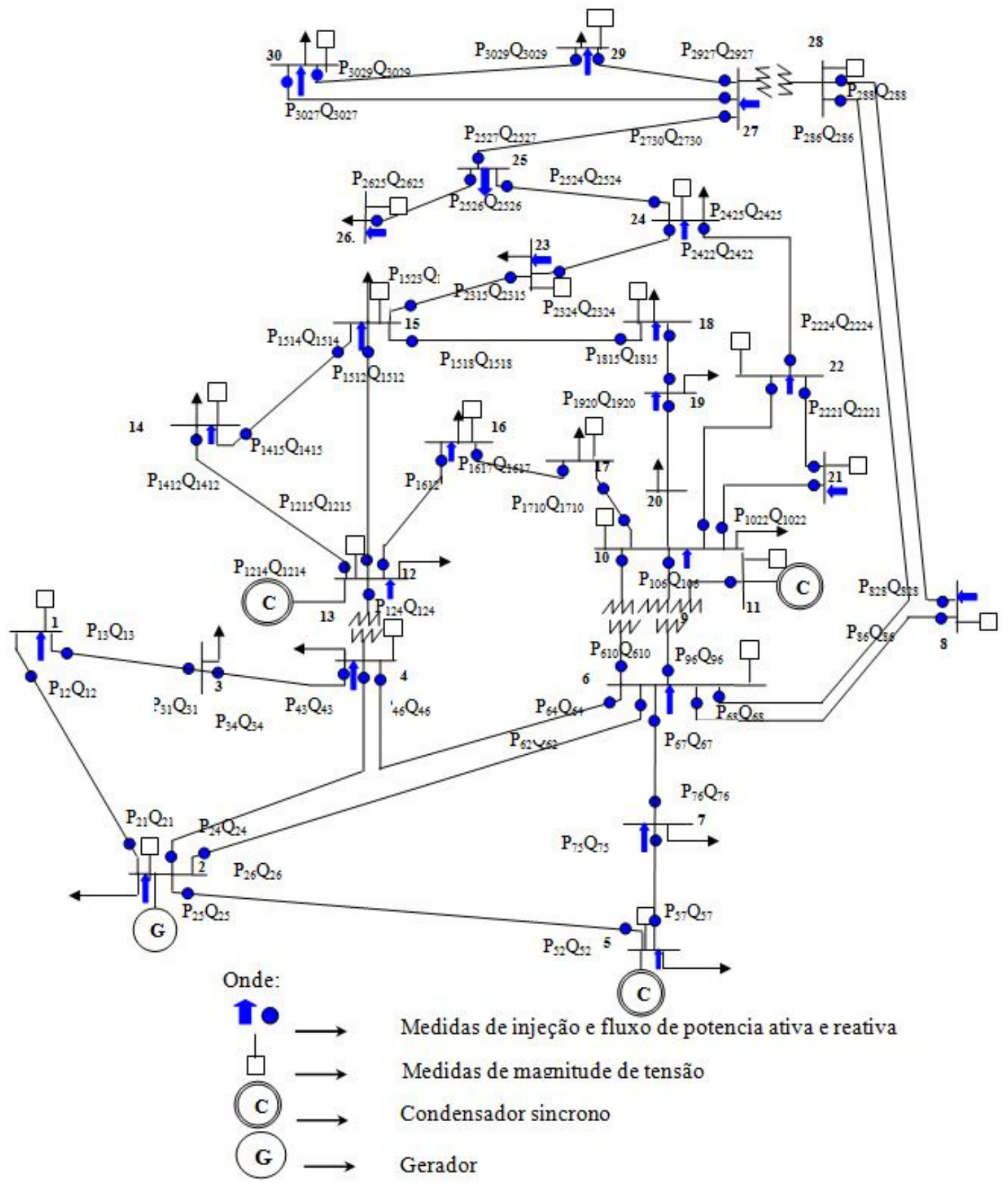

Figura 5.3: Sistema de 30 barras do IEEE

Tabela 5.27: Parâmetros de linha do sistema de 30 barras do IEEE

\begin{tabular}{|c|c|c|c|c|c|}
\hline Condutância & $\begin{array}{c}\text { Valores } \\
\text { corretos }\end{array}$ & Suceptância & $\begin{array}{c}\text { Valores } \\
\text { verdadeiros }\end{array}$ & Suceptância shunt & Valores corretos \\
\hline $\mathbf{G}_{\mathbf{1 2}}$ & 5,2246 & $\mathbf{B 1 2}$ & $-15,6467$ & $\mathbf{B}^{\text {sh }} \mathbf{1 2}$ & 0,0264 \\
\hline $\mathbf{G 1 3}$ & 1,5408 & $\mathbf{B 1 3}$ & $-5,6316$ & $\mathbf{B}^{\text {sh }} \mathbf{1 3}$ & 0,0204 \\
\hline
\end{tabular}




\begin{tabular}{|c|c|c|c|c|c|}
\hline G24 & 1,7055 & B24 & $-5,1973$ & $B^{\text {sh }} 24$ & 0,0184 \\
\hline G25 & 1,1359 & B25 & $-4,7724$ & $B^{\text {sh }} 25$ & 0,0209 \\
\hline G26 & 1,6861 & B26 & $-5,1164$ & $B^{\text {sh }} 26$ & 0,0187 \\
\hline G34 & 8,1954 & B34 & $-23,5308$ & $\mathrm{~B}^{\mathrm{sh}} 34$ & 0,0042 \\
\hline G46 & 6,4131 & B46 & $-22,3112$ & $B^{\text {sh }} 46$ & 0,0045 \\
\hline G412 & 0 & B412 & $-3,9063$ & $B^{\text {sh }} 412$ & 0 \\
\hline G57 & 2,9540 & B57 & $-7,4492$ & $B^{\text {sh }} 57$ & 0,0102 \\
\hline G67 & 3,5902 & B67 & $-11,0261$ & $B^{\text {sh } 67}$ & 0,0085 \\
\hline G68 & 6,2893 & B68 & $-22,0125$ & $B^{\text {sh } 68}$ & 0,0045 \\
\hline G69 & 0 & B69 & $-4,8076$ & $\mathbf{B}^{\text {sh }} 69$ & 0 \\
\hline G610 & 0 & B610 & $-1,7985$ & $B^{\text {sh } 610}$ & 0 \\
\hline G628 & 4,3628 & B628 & $-15,4635$ & $\mathrm{~B}^{\text {sh }} 628$ & 0,0065 \\
\hline G828 & 1,4439 & B828 & $-4,5408$ & $B^{\text {sh }} 828$ & 0,0214 \\
\hline G911 & 0 & B911 & $-4,8076$ & B $^{\text {sh }} 911$ & 0 \\
\hline G910 & 0 & B910 & $-9,0909$ & B $^{\text {sh }} 910$ & 0 \\
\hline G1020 & 1,7848 & B1020 & $-3,9853$ & $\mathrm{~B}^{\mathrm{sh}} \mathbf{1 0 2 0}$ & 0 \\
\hline G1017 & 3,9560 & B1017 & $-10,3174$ & $\mathrm{~B}^{\mathrm{sh}} \mathbf{1 0 1 7}$ & 0 \\
\hline G1021 & 5,1018 & B1021 & $-10,9807$ & $B^{\text {sh }} 1021$ & 0 \\
\hline G1022 & 2,6193 & B1022 & $-5,4008$ & $\mathrm{~B}^{\mathrm{sh}} 1022$ & 0 \\
\hline G1213 & 0 & B1213 & $-7,1429$ & $\mathrm{~B}^{\mathrm{sh}} 1213$ & 0 \\
\hline G1214 & 1,5266 & B1214 & $-3,1734$ & $B^{\text {sh }} 1214$ & 0 \\
\hline G1215 & 3,0954 & B1215 & $-6,0973$ & $\mathrm{~B}^{\mathrm{sh}} 1215$ & 0 \\
\hline G1216 & 1,9520 & B1216 & $-4,1044$ & $\mathrm{~B}^{\mathrm{sh}} 1216$ & 0 \\
\hline G1415 & 2,4909 & B1415 & $-2,2508$ & $\mathrm{~B}^{\mathrm{sh}} 1415$ & 0 \\
\hline G1518 & 1,8108 & B1518 & $-3,6874$ & $\mathrm{~B}^{\mathrm{sh}} 1518$ & 0 \\
\hline G1523 & 1,9683 & B1523 & $-3,9760$ & $B^{\text {sh }} 1523$ & 0 \\
\hline G1617 & 1,3190 & B1617 & $-4,8407$ & $B^{\text {sh }} 1617$ & 0 \\
\hline G1819 & 3,0757 & B1819 & $-6,2187$ & $\mathrm{~B}^{\mathrm{sh}} 1819$ & 0 \\
\hline G1920 & 5,8823 & B1920 & $-11,7647$ & $\mathrm{~B}^{\mathrm{sh}} \mathbf{1 9 2 0}$ & 0 \\
\hline G2122 & 16,7746 & B2122 & $-34,1277$ & $B^{\text {sh }} 2122$ & 0 \\
\hline G2224 & 2,5405 & B2224 & $-3,9544$ & $\mathrm{~B}^{\mathrm{sh}} 2224$ & 0 \\
\hline G2324 & 1,4614 & B2324 & $-2,9892$ & $\mathrm{~B}^{\mathrm{sh}} 2324$ & 0 \\
\hline G2425 & 1,3099 & B2425 & $-2,2876$ & $\mathrm{~B}^{\mathrm{sh}} 2425$ & 0 \\
\hline G2526 & 1,2165 & B2526 & $-1,8171$ & $\mathrm{~B}^{\text {sh } 2526}$ & 0 \\
\hline G2527 & 1,9693 & B2527 & $-3,7602$ & B $^{\text {sh }} 2527$ & 0 \\
\hline G2729 & 0,9955 & B2729 & $-1,8810$ & $B^{\text {sh } 2729}$ & 0 \\
\hline G2730 & 0,6874 & B2730 & $-1,2939$ & $\mathrm{~B}^{\text {sh } 2730}$ & 0 \\
\hline G2728 & 0 & B2728 & $-2,5252$ & $B^{\text {sh }} 2728$ & 0 \\
\hline G2930 & 0,9129 & B2930 & $-1,7234$ & $\mathrm{~B}^{\text {sh } 2930}$ & 0 \\
\hline
\end{tabular}

\section{Teste 8:}

Neste teste será analisada a capacidade de a metodologia proposta obter boas estimativas para diferentes porcentagens de erros, nos parâmetros de um determinado ramo, assim como foi realizado em (LIU;WU; LUN, 1992).

O desempenho da metodologia proposta será avaliada quanto à variação do erro, nos seguintes parâmetros: 
Situação 1: erro na susceptância série da linha entre as barras 10 e 20 (os demais parâmetros do sistema sem erro). Os resultados estão na tabela 5.28;

Situação 2: erro na susceptância série da linha entre as barras 2 e 4 (os demais parâmetros do sistema sem erro). Os resultados estão na tabela 5.29;.

Tabela 5.28 Resultados referentes à Situação 1 (metodologia proposta)

\begin{tabular}{|c|c|c|c|c|}
\hline \multicolumn{5}{|c|}{ Valor verdadeiro b=-3,9854 } \\
\hline Caso & Condição inicial & $\begin{array}{c}\text { \% error } \\
\text { (original) }\end{array}$ & $\begin{array}{c}\text { Valor estimado } \\
\text { (b) }\end{array}$ & $\begin{array}{c}\text { \% de erro nos } \\
\text { valores estimados }\end{array}$ \\
\hline $\mathbf{1}$ & $-3,7850$ & 5 & $-3,9622$ & 0,5821 \\
\hline $\mathbf{2}$ & $-3,5850$ & 10 & $-3,9620$ & 0,5871 \\
\hline $\mathbf{3}$ & $-3,1850$ & 20 & $-3,9621$ & 0,5846 \\
\hline $\mathbf{4}$ & $-2,3850$ & 40 & $-3,9620$ & 0,5871 \\
\hline $\mathbf{5}$ & $-0,7850$ & 80 & $-3,9619$ & 0,5897 \\
\hline
\end{tabular}

Tabela 5.29 Resultados referentes à Situação 2 (metodologia proposta)

\begin{tabular}{|c|c|c|c|c|}
\hline \multicolumn{5}{|c|}{ Valor verdadeiro b=-5,1974 } \\
\hline Caso & Condição inicial & $\begin{array}{c}\text { \% error } \\
\text { (original) }\end{array}$ & $\begin{array}{c}\text { Valor estimado } \\
\text { (b) }\end{array}$ & $\begin{array}{c}\text { \% de erro nos } \\
\text { valores estimados }\end{array}$ \\
\hline $\mathbf{1}$ & $-5,4570$ & 5 & $-5,1922$ & 0,1001 \\
\hline $\mathbf{2}$ & $-5,7170$ & 10 & $-5,1919$ & 0,1058 \\
\hline $\mathbf{3}$ & $-6,2380$ & 20 & $-5,1920$ & 0,1039 \\
\hline $\mathbf{4}$ & $-7,2740$ & 40 & $-5,1921$ & 0,1020 \\
\hline $\mathbf{5}$ & $-9,3870$ & 80 & $-5,1919$ & 0,1058 \\
\hline
\end{tabular}

É importante observar que a metodologia proposta permite corrigir significativamente o erro no parâmetro, mesmo na presença de um erro de até $80 \%$.

Nas Tabelas 5.30 e 5.31, acham-se os resultados da aplicação do estimador de parâmetros proposto em (LIU; WU; LUN, 1992), para análise das situações 1 e 2, respectivamente. Vale destacar que, conforme mencionado no capitulo 3, em (LIU; WU; LUN, 1992) propõe-se a estimação dos EPs, através da análise do vetor de resíduo, e, através dessas estimativas, realiza-se a correção dos parâmetros com erros.

Tabela 5.30 Resultados referentes à Situação 1 (estimador proposto em (LIU; WU;LUN, 1992))

\begin{tabular}{|c|c|c|c|c|}
\hline \multicolumn{5}{|c|}{ Valor verdadeiro b=-3,9854 } \\
\hline Caso & Condição inicial & $\begin{array}{c}\text { \% error } \\
\text { (original) }\end{array}$ & $\begin{array}{c}\text { Valor estimado } \\
(\mathbf{b})\end{array}$ & $\begin{array}{c}\text { \% de erro nos } \\
\text { valores estimados }\end{array}$ \\
\hline $\mathbf{1}$ & $-3,7850$ & 5 & $-3,9700$ & 0,38 \\
\hline $\mathbf{2}$ & $-3,5850$ & 10 & $-3,9500$ & 0,88 \\
\hline $\mathbf{3}$ & $-3,1850$ & 20 & $-3,8930$ & 2,30 \\
\hline $\mathbf{4}$ & $-2,3850$ & 40 & $-3,6850$ & 7,51 \\
\hline $\mathbf{5}$ & $-0,7850$ & 80 & $-2,5310$ & 36,47 \\
\hline
\end{tabular}


Tabela 5.31 Resultados referentes à Situação 2 (estimador proposto em (LIU;WU;LUN, 1992))

\begin{tabular}{|c|c|c|c|c|}
\hline \multicolumn{5}{|c|}{ Valor verdadeiro b=5,1974 } \\
\hline Caso & Condição inicial & $\begin{array}{c}\text { \% error } \\
\text { (original) }\end{array}$ & $\begin{array}{c}\text { Valor estimado } \\
\text { (b) }\end{array}$ & $\begin{array}{c}\text { \% de erro nos } \\
\text { valores estimados }\end{array}$ \\
\hline $\mathbf{1}$ & $-5,4570$ & 5 & $-5,1920$ & 0,10 \\
\hline $\mathbf{2}$ & $-5,7170$ & 10 & $-5,1870$ & 0,19 \\
\hline $\mathbf{3}$ & $-6,2380$ & 20 & $-5,1760$ & 0,43 \\
\hline $\mathbf{4}$ & $-7,2740$ & 40 & $-5,1480$ & 0,96 \\
\hline $\mathbf{5}$ & $-9,3870$ & 80 & $-5,0820$ & 2,23 \\
\hline
\end{tabular}

\section{Teste 9: Erros nos parâmetros de 3 ramos (sistema com medidas redundantes)}

Neste teste, EPs múltiplos são adicionados simultaneamente aos parâmetros de ramos 1-2, 2-4 e 8-28. Observe que os ramos 1-2 e 2-4 são adjacentes. A tabela 5.32 apresenta os valores corretos, os valores iniciais e os valores estimados do parâmetros daqueles ramos. Os valores de todos os parâmetros estimados foram corrigidos, principalmente a suceptância serie do ramo 8-28 (de 30\% a menor que $0.2923 \%$ de erro).

Tabela 5.32: Parâmetros estimados do teste 9

\begin{tabular}{|c|c|c|c|c|c|}
\cline { 2 - 6 } \multicolumn{1}{c|}{} & Inicial* & Estimado & Corretos & $\begin{array}{c}\text { \% de erro nos } \\
\text { valores iniciais }\end{array}$ & $\begin{array}{c}\text { \% de erro nos valores } \\
\text { estimados }\end{array}$ \\
\hline $\mathrm{G}_{1-2}$ & 6,7919 & 5,2530 & 5,2246 & 30,0 & 0,5436 \\
\hline $\mathrm{B}_{1-2}$ & $-20,3407$ & $-15,5596$ & $-15,6467$ & 30,0 & 0,5566 \\
\hline$B_{1-2}^{\text {shunt }}$ & 0,0343 & 0,0263 & 0,0264 & 30,0 & 0,3787 \\
\hline $\mathrm{G}_{2-4}$ & 2,2171 & 1,7001 & 1,7055 & 30,0 & 0,3166 \\
\hline $\mathrm{B}_{2-4}$ & 6,7565 & $-5,2177$ & $-5,1973$ & 30,0 & 0,3925 \\
\hline$B_{2-4}^{\text {shunt }}$ & 0,02392 & 0,0183 & 0,0184 & 30,0 & 0,5434 \\
\hline $\mathrm{G}_{8-28}$ & 1,8770 & 1,4590 & 1,4439 & 30,0 & 1,0457 \\
\hline $\mathrm{B}_{8-28}$ & $-5,9030$ & $-4,5570$ & $-4,5408$ & 30,0 & 0,2923 \\
\hline$B_{8-28}^{\text {shunt }}$ & 0,0278 & 0,0216 & 0,0214 & 30,0 & 0,9345 \\
\hline
\end{tabular}

\subsection{ANÁLISE DOS RESULTADOS}

Apreciando os resultados obtidos em todos os testes realizados, permite-se-nos afirmar que: 
- A metodologia proposta possibilita boas estimativas, mesmo na presença de um ou mais erros nos parâmetros série de uma ou mais linhas de transmissão, mesmo sendo em linhas adjacentes;

- Através da metodologia proposta é possível obter boas estimativas para diferentes porcentagens de erro em um parâmetro e na presença de medidas com ruído;

- Comparando os resultados obtidos pela metodologia proposta, constantes das Tabelas 5.30 e 5.31, com os obtidos pelo estimador desenvolvido em (LIU; WU; LUN, 1992), apresentados nas Tabelas 5.28 e 5.29, comprova-se a viabilidade daquela metodologia. Vale destacar, entretanto, que, tomando por base esses resultados, não podemos afirmar que a nossa metodologia seja superior ao estimador desenvolvido em (LIU; WU;LUN, 1992). Isto porque, em razão de não ter sido apresentado o conjunto de medidas utilizado naquela referência, não é possível realizar uma comparação mais justa entre as duas metodologias de estimação de parâmetros. Contudo, de qualquer forma, já é um indicativo da viabilidade da metodologia proposta. 


\section{Capítulo 6}

\section{TESTES REALIZADOS COM O SISTEMA DA HYDRO-QUÉBEC TRANS-ÈNERGIE}

Neste capítulo serão apresentados alguns dos resultados da aplicação da metodologia proposta em dois subsistemas da empresa Hydro-Québec, ambos monitorados pelo centro de controle regional (CCR) da TransÉnergie ${ }^{16}$, localizado em Québec. Esses subsistemas foram utilizados tendo em vista que os mesmos foram considerados suspeitos de estarem com EPs, quando analisados pela metodologia desenvolvida em (VOUNG; LEFEBVRE, 2002).

Foram contempladas situações que permitiram avaliar as três fases que compõe a metodologia proposta.

\subsection{SUBSISTEMAS ANALISADOS}

O primeiro subsistema (subsistema 1), cujo diagrama unifilar é mostrado na Figura 6.1, possui 3 barras e 3 linhas de transmissão de $315 \mathrm{kV}$ : a primeira com $46 \mathrm{~km}$ conectando as subestações 315-3 e 315-2 (ramo L315-3); a segunda com $83 \mathrm{~km}$ conectando as subestações 315-2 E 315-1(ramo L315-2); e a terceira com 130km conectando as subestações 315-3 e 315-1 (ramo L315-1). Como pode ser visto na Figura 6.1, em termos de sistema de medição o subsistema 1 possui medidores de magnitude de tensão em todas as 3 barras e pares de fluxos de potencia ativa e reativa em todos os terminais das três linhas, resultando num total de 15 medidores.

16 TransÉnergie é uma divisão da Hydro-Québec. 


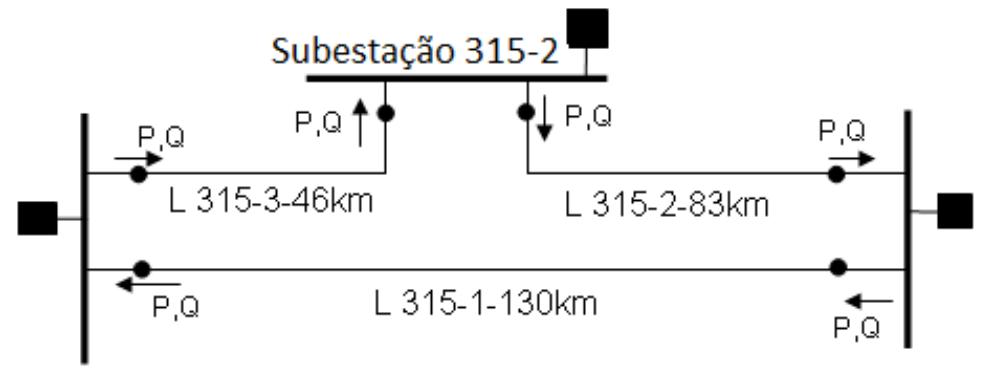

Subestação 315-3

Subestação $315-1$

Onde:

- - Pares de medidas de fluxo de Potência

(ativa e reativa)

- Medidas de Magnitude de Tensão

Figura 6.1. Subsistema 1

Para destacar a importância do subsistema 1, importa ressaltar que o mesmo está sendo analisado pela equipe de pesquisa do IREQ (Instituto de Recherche d'Hydro-Québec), no projeto de pesquisa e desenvolvimento intitulado "REPORTER", cujo objetivo é desenvolver uma ferramenta de auxilio, ao operador do Sistema, para detecção de anomalias no estimador de estado comercial.

O segundo subsistema (subsistema 2), cujo diagrama unifilar é mostrado na Figura 6.2, é um corredor de energia muito importante para Hydro-Québec possuindo 2 barras (subestações 735-1 e 735-2) e três linhas de transmissão paralelas de 735kv. Esse sistema foi utilizado para mostrar que a metodologia proposta possibilita a estimação de parâmetros de linhas paralelas através de medidas obtidas nos extremos das mesmas.

Conforme ilustrado na Figura 6.2, em termos de sistema de medição o subsistema 2 possui medidores de magnitude de tensão nas 2 barras e pares de medidores de fluxos de potencia ativa e reativa em todos os terminais das três linhas, resultando num total de 14 medidores. Uma particularidade desse subsistema é que o mesmo possui compensação shunt nas duas extremidades das linhas e compensação série (banco de capacitores) onde (Xc é conhecido), ponto $\mathrm{R}$ na Figura 6.2, localizada a $242 \mathrm{~km}$ da subestação $735-1$ e a $137 \mathrm{~km}$ da subestação 735-2. Em razão dessa compensação, as linhas serão representadas por 6 ramos (L735-1A, L735-2A, L735-3A L735-1B, L735-2B, L735-3B ). 


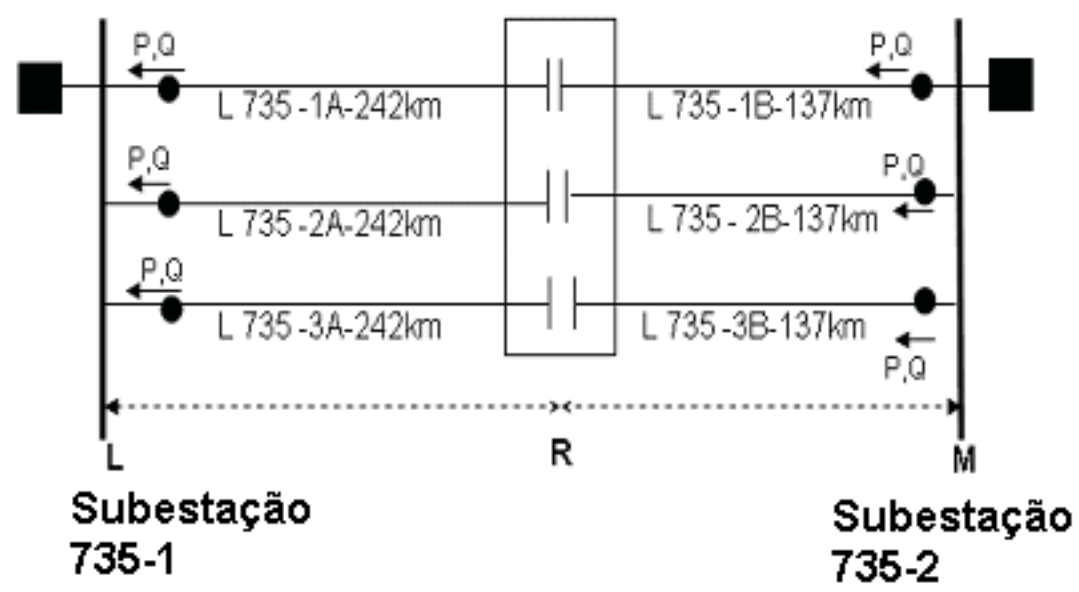

Onde:

- - Pares de medidas de fluxo de Potência (ativa e reativa)

$\boldsymbol{\varphi}$ - Medidas de Magnitude de Tensão

Figura 6.2. Subsistema 2

Para possibilitar a aplicação da metodologia proposta para esse subsistema, cada uma das suas linhas foi representada de acordo com a Figura 6.3 (YUAN LIAO, 2009).

Na Figura 6.3 adotam-se as seguintes notações: $Z_{A}, Z_{B}$ impedâncias série equivalentes dos segmentos de linha LR e RM, respectivamente; $Y_{A}, Y_{B}$ admitâncias shunt equivalentes dos segmentos de linha LR e RM, respectivamente; e $X_{C}$ a reatância capacitiva do banco de capacitores (desconhecida).

Observando a Figura 6.3, verifica-se que o segmento de linha LR, do lado esquerdo do dispositivo de compensação, pode ser analisado como um circuito delta A. Da mesma forma, o segmento de linha RM pode ser analisado como um circuito delta B. Conseqüentemente, aplicando sucessivamente transformações delta-estrela e estrela-delta, para ambos os circuitos delta $\mathrm{A}$ e delta $\mathrm{B}$, obteve-se um modelo $\pi$-equivalente para cada uma das três linhas de transmissão do subsistema 2.

A metodologia proposta foi então aplicada para estimar os parâmetros do modelo $\pi$ equivalente de cada uma das três linhas de transmissão. Conseqüentemente, para cada 
amostra de medidas, processadas pela metodologia proposta, foram estimadas 12 variáveis, isto é, 9 parâmetros de linha (condutância, suceptância serie e susceptancia shunt para cada uma das três linhas), duas magnitudes de tensão (nas subestações 735-1 e 735-2) e um ângulo de fase de tensão (na subestação 735-2). Depois da estimação dos parâmetros de cada uma das três linhas, calcularam-se os parâmetros das linhas correspondentes a cada um dos segmentos LR e RM. Para isso, basta seguir, na ordem inversa, o processo descrito acima.

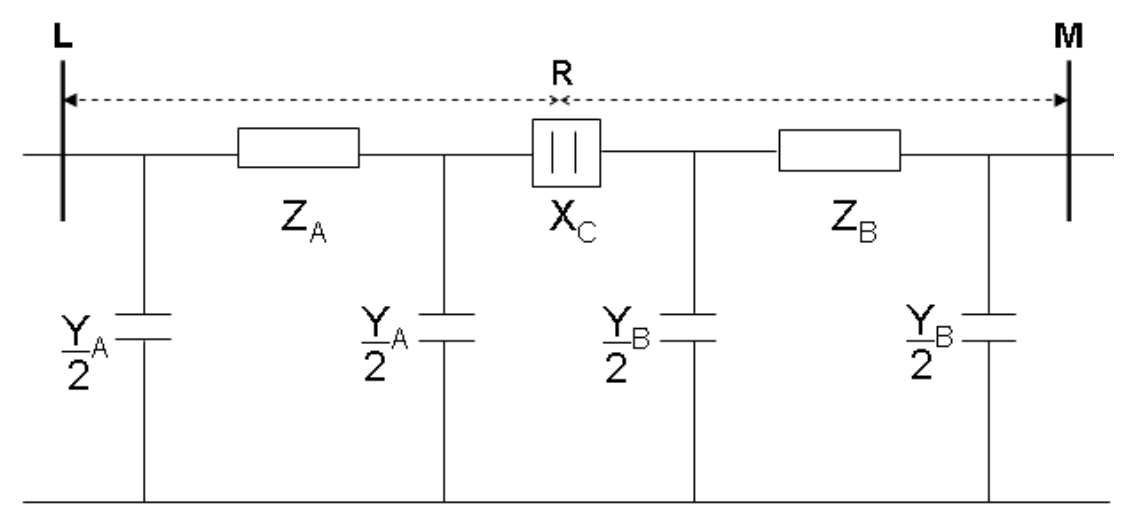

Figura 6.3 Representação de uma linha do subsistema 2

\subsection{AMOSTRAS UTILIZADAS}

As amostras de medidas utilizadas nos testes foram fornecidas pelo CCR da TransÉnergie, tendo sido coletadas em intervalos de 5 minutos para dias típicos, com condições normais de operação. De acordo com a experiência do operador do sistema, as amostras selecionadas estão livres de EGs e topológicos. Adotaram-se os seguintes valores de desvios padrão $(\sigma)$ (usados para a ponderação das medidas): $\sigma=0.05$ p.u. para medidas de potencia ativa; $\sigma=0.15$ p.u.para medidas de potencia reativa; e $\sigma=0.01$ p.u. para medidas de magnitude de tensão.

\subsection{VALIDAÇÃO DOS RESULTADOS OBTIDOS}

Como os valores dos parâmetros verdadeiros não são conhecidos, para validar os resultados obtidos pela metodologia off-line proposta, o índice de desempenho do WLS 
$J(x)=\sum_{i=0}^{m} r_{i}^{t} W r_{i}$, será calculado para várias amostras de medidas, considerando ora os valores dos parâmetros disponíveis no banco de dados da empresa e ora os valores estimados. Para isso, foi utilizado um estimador de estado comercial, disponível no CCR de TransÉnergie, denominado LASER 2. As estimativas dos parâmetros foram validadas porque o correspondente índice de desempenho $\mathrm{J}(\mathrm{x})$ foi menor que o obtido através dos valores dos parâmetros disponíveis na empresa.

A avaliação da qualidade da solução do estimador de estado na Hydro-Québec é analizada através de duas métricas $J_{T}=M-2 N$ e $J_{M}=\sum_{i=1}^{m} r_{W_{i}}^{2}$

\subsection{Teste - Subsistema 1}

Aplicando a metodologia proposta obteve-se (veja o fluxograma ilustrado na Figura 4.2, capítulo 4):

Fase 1: Identificação de ramos suspeitos: o resultado da aplicação desta fase é a lista de ramos suspeitos apresentada na Tabela 6.1. Destacam-se, na coluna 5 dessa tabela, os valores dos maiores resíduos normalizados $\left(\mathrm{r}^{\mathrm{N}}\right)$, em módulo, entre as medidas de cada TM(i).

Observação 6.1: Nas próximas tabelas utilizar-se-á a seguinte nomenclatura: $\mathrm{P}_{\mathrm{a}-\mathrm{b}}\left(\mathrm{Q}_{\mathrm{a}-\mathrm{b}}\right)$ medida de fluxo de potencia ativa (reativa) da barra "a" para barra "b"; $G_{a}$ - condutância série do ramo "a"; $B_{\mathrm{a}}$ - susceptância serie do ramo "a"; e $B_{a}^{\text {shunt }}$ - susceptância shunt do ramo "a".

Tabela 6.1: Primeira lista de ramos suspeitos - Subsistema 1

\begin{tabular}{|l|c|c|c|c|c|}
\hline \multicolumn{1}{|c|}{ Ramo i } & $\begin{array}{c}\text { TM( } \\
\text { i) }\end{array}$ & MRE(i) & Medidas em MRE(i) & Maior $\mathrm{r}^{\mathrm{N}}$ & IS (i) \\
\hline L315-1(1-3) & 6 & 2 & $\mathrm{~F}::_{1-3}, \mathrm{~F}: 3-1$, & 6,22 & 0,33 \\
\hline L315-2: (1-2) & 6 & 2 & $\mathrm{~F}: 1-2, F: 2-1$ & 4,62 & 0,33 \\
\hline L315-3: (2-3) & 6 & 2 & $\mathrm{~F}: 3-2, F:_{2-3}$ & 4,40 & 0,33 \\
\hline
\end{tabular}

De acordo com a Tabela 6.1, todos os ramos têm o mesmo IS. O ramo L315-1 é considerado como o mais suspeitos tendo em vista que a medida com maior resíduo normalizado é incidente a esse ramo. Ramo L315-2 é selecionado para ter seus parâmetros estimados. 
Fase 2: Estimação de parâmetros suspeitos: $\mathrm{Na}$ Tabela 6.2 encontram-se os valores disponíveis no banco de dados (iniciais) e os estimados do ramo L315-1. O critério de parada foi satisfeito na oitava amostra de medidas (as amostras selecionadas são do dia 1 de abril de 2009, dás 2:10 hs as 3:00 hs).

Tabela 6.2: Resultados Parciais - Subsistema 1

\begin{tabular}{|c|c|c|c|}
\cline { 2 - 4 } \multicolumn{1}{c|}{} & Inicial* & Estimado & \% de correção \\
\hline $\mathrm{G}_{\mathrm{L} 315-1}$ & 2,5416 & 2,4629 & 3,09 \\
\hline $\mathrm{B}_{\mathrm{L} 315-1}$ & $-27,7629$ & $-45,5725$ & 64,14 \\
\hline$B_{\text {L315-1 }}^{\text {shunt }}$ & 0,81757 & 0,79080 & 3,27 \\
\hline & * Valores dos parâmetros disponíveis na base de dados \\
\hline
\end{tabular}

Fase 3: Validação das estimativas dos parâmetros suspeitos: validam-se as estimativas. Em seguida realizam-se as seguintes tarefas: a base de dados é atualizada, isto é, os valores disponíveis dos parâmetros do ramo L315-1 são substituídos pelos valores estimados; a lista de ramos suspeitos é esvaziada (LSB = $\phi$ ); e o ramo L315-1 é armazenado na lista de ramos suspeitos cujos parâmetros já foram estimados e validados (LSBEV $\leftarrow$ L315-1).

Fase 1': Identificação de ramos suspeitos: o resultado da aplicação desta fase encontra-se na Tabela 6.3. Ramo L315-3 é selecionado para ser estimado.

Tabela 6.3: Segunda Lista de Ramos Suspeitos - Subsistema 1

\begin{tabular}{|c|c|c|c|c|c|}
\hline Ramo i & TM(i) & MRE(i) & Medidas em MRE(i) & ${\text { Maior } \mathrm{r}^{\mathrm{N}}}$ & IS (i) \\
\hline L315-3: (2-3) & 6 & 2 & $\mathrm{Q}_{3-2}, \mathrm{Q}_{22-3}$ & 5,95 & 0,33 \\
\hline L315-2: (1-2) & 6 & 1 & $\mathrm{Q}_{1-2}$ & 4,45 & 0,17 \\
\hline
\end{tabular}

Fase 2': Estimação de parâmetros suspeitos: Na Tabela 6.4 encontram-se os valores disponíveis no banco de dados (iniciais) e os estimados do ramo L315-3. O critério de parada foi satisfeito na quarta amostra de medida (as amostras selecionadas são do dia 1 de abril de 2009, dás 2:10 hs as 3:00 hs).

Tabela 6.4: Resultados Finais - Subsistema 1

\begin{tabular}{|c|c|c|c|}
\cline { 2 - 4 } \multicolumn{1}{c|}{} & Inicial* $^{*}$ & Estimado & \% de correções \\
\hline $\mathrm{G}_{\mathrm{L} 315-3}$ & 4,1689 & 4,2860 & 2,80 \\
\hline
\end{tabular}




\begin{tabular}{|c|c|c|c|}
\hline $\mathrm{B}_{\mathrm{L} 315-3}$ & $-44,9027$ & $-43,9296$ & 2,16 \\
\hline$B_{\text {L315-3 }}^{\text {shunt }}$ & 0,16426 & 0,15480 & 5,76 \\
\hline
\end{tabular}

Fase 3': Validação das estimativas dos parâmetros suspeitos: validam-se as estimativas. Em seguida realizam-se as seguintes tarefas: a base de dados é atualizada, isto é, os valores disponíveis dos parâmetros do ramo L315-3 são substituídos pelos valores estimados; a lista de ramos suspeitos é esvaziada (LSB $=\phi$ ); e o ramo L315-3 é armazenado na lista de ramos suspeitos cujos parâmetros já foram estimados e validados (LSBEV $\leftarrow$ L315-3).

Fase 1": Identificação de ramos suspeitos: agora, considerando os parâmetros estimados, um estimador por MQP convencional é executado. Como não há nenhuma medida com resíduo normalizado maior que 3 , a analise é finalizada. Observe que, de acordo a metodologia proposta, os valores dos parâmetros do ramo L315-2, disponíveis na base de dados da empresa, estão corretos.

Validação das Estimativas - Subsistema 1

Para validar os parâmetros estimados, calculou-se o índice de desempenho $\mathrm{J}(\mathrm{x})$, considerando os valores disponíveis da empresa $\left(\mathrm{J}^{1}(\mathrm{x})\right)$ e os valores dos parâmetros estimados $\left(\mathrm{J}^{2}(\mathrm{x})\right)$, para cada uma das amostras de medidas obtidas a cada 5 minutos no dia 24 de setembro de 2009. A Figura 6.4 apresenta os valores calculados para relação $J^{1}(X) / J^{2}(X)$. Observe que para todos os pontos de operação essa relação é maior que 1 , validando as estimativas dos parâmetros.

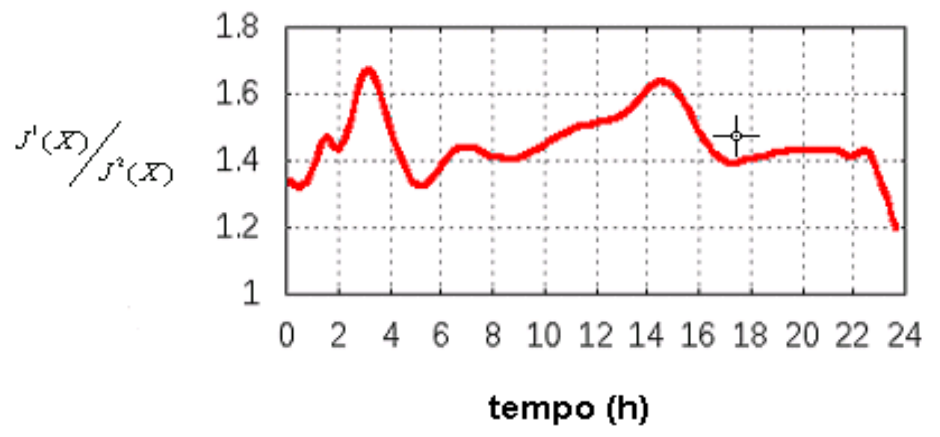

Figura 6.4. Relação dos índices de desempenho obtidos a partir de amostras de medidas do dia 24 de setembro de $2009 J^{1}(X) / J^{2}(X)$ - Subsistema 1. 
Para validar as estimativas obtidas foram utilizadas, também, amostras de medidas de outros dias.

\subsection{Teste - Subsistema 2}

Os resultados obtidos encontram-se na Tabela 6.5. Importa destacar que, em razão das compensações reativas do subsistema 2 , os valores das medidas de potência reativa obtidas em amostras consecutivas são bem diferentes. Assim, para aplicar a metodologia proposta utilizou-se apenas uma amostra de medida (obtida às 2 horas e 29 minutos do dia 7 de agosto de 2009).

Tabela 6.5: Resultados - Subsistema 2

\begin{tabular}{|c|c|c|c|}
\hline & Inicial* & Estimados & \% de correção \\
\hline $\mathrm{G}_{\mathrm{L} 735-1 \mathrm{~A}}$ & 2,1947 & 2,3101 & 5,2581 \\
\hline $\mathrm{B}_{\mathrm{L} 735-1 \mathrm{~A}}$ & 65,2430 & 67,1438 & 2,9134 \\
\hline$B_{L 735-1 A}^{\text {shunt }}$ & 6,3188 & 6,5931 & 4,3410 \\
\hline $\mathrm{G}_{\mathrm{L} 735-1 \mathrm{~B}}$ & 3,8536 & 4,0549 & 5,2237 \\
\hline $\mathrm{B}_{\mathrm{L} 735-1 \mathrm{~B}}$ & $-115,2113$ & $-118,5683$ & 2,9137 \\
\hline$B_{L 735-1 B}^{\text {shunt }}$ & 3,61504 & 3,7719 & 4,34 \\
\hline $\mathrm{G}_{\mathrm{L} 735-2 \mathrm{~A}}$ & 2,1902 & 0,8656 & 60,4784 \\
\hline $\mathrm{B}_{\mathrm{L} 735-2 \mathrm{~A}}$ & $-64,9879$ & $-65,0232$ & 0,0543 \\
\hline$B_{L 735-2 A}^{\text {shunt }}$ & 6,35387 & 6,8313 & 7,5146 \\
\hline $\mathrm{G}_{\mathrm{L} 735-2 \mathrm{~B}}$ & 3,9356 & 1,5550 & 60,4888 \\
\hline $\mathrm{B}_{\mathrm{L} 735-2 \mathrm{~B}}$ & $-117,6539$ & $-117,7165$ & 0,0532 \\
\hline$B_{L 735-2 B}^{\text {shunt }}$ & 3,54878 & 3,8154 & 7,5146 \\
\hline $\mathrm{G}_{\mathrm{L} 735-3 \mathrm{~A}}$ & 2,8416 & 2,8087 & 1,1577 \\
\hline $\mathrm{B}_{\mathrm{L} 735-3 \mathrm{~A}}$ & $-66,9935$ & $-67,3574$ & 0,5431 \\
\hline$B_{L 735-3 A}^{\text {shunt }}$ & 6,36856 & 6,74776 & 5,9542 \\
\hline $\mathrm{G}_{\mathrm{L} 735-3 \mathrm{~B}}$ & 5,2357 & 5,1747 & 1,1650 \\
\hline $\mathrm{B}_{\mathrm{L} 735-3 \mathrm{~B}}$ & $-119,8192$ & $-120,4705$ & 0,5435 \\
\hline$B_{L 735-3 B}^{\text {shunt }}$ & 3,58253 & 3,79643 & 5,9706 \\
\hline \multicolumn{4}{|c|}{ * Valores dos Parâmetros disponíveis na base de dados } \\
\hline
\end{tabular}

Validação das Estimativas - Subsistema 2

Para validar os parâmetros estimados, calculou-se o índice de desempenho J(x), considerando os valores disponíveis da empresa $\mathrm{J}^{1}(\mathrm{x})$ e os valores dos parâmetros 
estimados $\mathrm{J}^{2}(\mathrm{x})$, para cada uma das amostras de medidas obtidas a cada 5 minutos no dia 26 de setembro de 2009. A Figura 6.6 apresenta os valores calculados para relação $J^{1}(X) / J^{2}(X)$. Observe que para todos os pontos de operação essa relação é maior que 1 , validando as estimativas dos parâmetros.

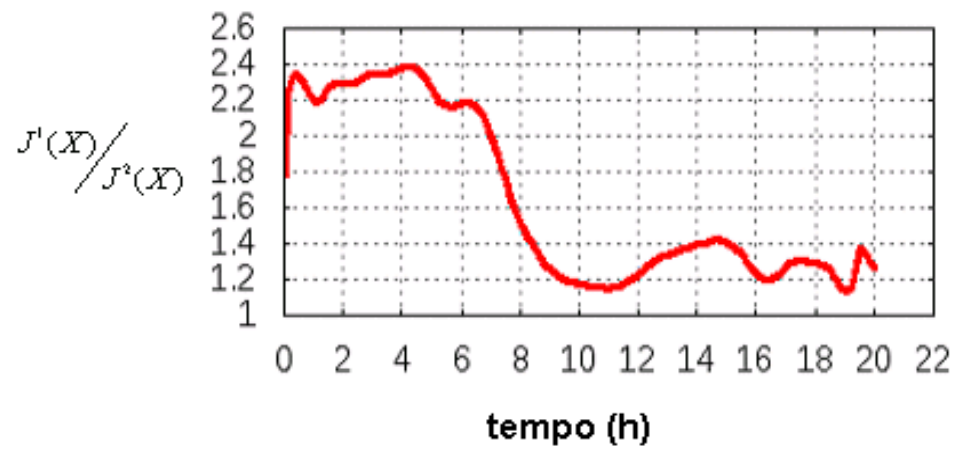

Figura 6.5. Relação dos índices de desempenho obtidos a partir de amostras de medidas do dia 26 de setembro $J^{1}(X) / J^{2}(X)$ - Subsistema 2.

\subsection{ANÁLISE DOS RESULTADOS}

Neste capítulo foram apresentados resultados de simulações com dois subsistemas reais, da empresa Hydro-Québec. Destacam-se, a seguir, alguns comentários relativos aos resultados obtidos:

- A metodologia proposta mostrou-se bastante simples, para aplicação, e eficaz para correção de erros simples e múltiplos;

- Garantindo uma redundância suficiente e a não existência de EGs e erros de topologia, a metodologia proposta conseguiu melhorar os valores dos parâmetros de dois subsistemas da Hydro-Québec;

- Os resultados obtidos pela metodologia proposta foram utilizados para corrigir os valores dos parâmetros disponíveis na base de dados do CCR da TransÉnergie;

- Os resultados obtidos com a utilização de dados reais mostraram que a metodologia proposta pode auxiliar no processo de implantação da função de estimação de estado, corrigindo ou reduzindo as incertezas presentes nos parâmetros. 


\section{Capítulo 7}

\section{CONCLUSÕES E TRABALHOS FUTUROS}

\subsection{Conclusões}

Este capítulo resume o trabalho apresentado e destaca as principais contribuições do mesmo. Além disso, considera algumas perspectivas de extensões da metodologia proposta.

Os SEP estão enfrentando desafios importantes nos últimos anos. Liberalização do mercado, novo quadro regulamentar, novas tecnologias de geração, redes enfocadas no cliente, aumento da demanda, geração distribuída, questões ambientais, segurança de fornecimento ou envelhecimento da infra-estrutura, são fatores motivadores que exigem uma ação.

A modelagem em tempo real é uma ferramenta fundamental na moderna operação dos SEP. Vários aplicativos destinados à análise da segurança da operação dependem da disponibilidade de modelos confiáveis obtidos a partir do processamento de informações adquiridas em tempo real. Um requisito para a obtenção de tais modelos é o conhecimento da topologia da rede que corresponde às condições efetivamente existentes, definidas pelas informações sobre os estados de chaves e disjuntores assim como parâmetros da base de dados. Erros nestes dados comprometem os resultados fornecidos pelos aplicativos executados subseqüentemente, tais como a estimação de estado, a análise de contingências e o fluxo de potência em tempo real.

Conforme apresentado no capítulo 3, a busca por métodos eficientes para identificação e correção de EPs, para efeito de EESEP, tem inspirado diversas pesquisas.

Desenvolvimentos recentes relacionados à identificação e estimação de parâmetros com o uso de medidores sincronizados fasoriais (PMUs) (ZHU; ABUR 2009) foram propostos recentemente. 
Com o objetivo de contribuir para a solução do problema de estimação de parâmetros em SEP, este trabalho de pesquisa propôs uma metodologia off-line para depuração (detecção, identificação e correção de erros) de parâmetros de linhas de transmissão que faz uso de diversas amostras de medidas.

Os parâmetros a serem depurados, pela metodologia proposta, são as admitâncias série e suceptância shunt de linhas de transmissão aéreas, classificadas como médias e longas (com comprimento acima de $80 \mathrm{~km}$ ).

A metodologia proposta foi apresentada no capítulo 4, e compreende três fases: Fase 1: Identificação de parâmetros suspeitos de estarem com erro; Fase 2: Estimação dos parâmetros suspeitos; e Fase 3: Validação dos valores estimados.

Vale destacar que a metodologia foi desenvolvida tomando por base os seguintes aspectos relevantes relacionados à questão de EPs no processo de EESEP :

- Erros nas condutâncias série são menos influentes para o processo de EESEP do que erros nas susceptâncias série de linhas de transmissão (ou ramos da rede);

- As susceptâncias série são menos sensíveis a variações climáticas do que as condutâncias série. Conseqüentemente, pode-se considerar que os parâmetros série de linhas de transmissão não sofram mudanças significativas de valor durante um determinado intervalo de tempo (ABUR; EXPOSITO, 2004);

- Para estimar parâmetros que permanecem constantes, durante um determinado intervalo de tempo, o melhor procedimento é realizar um processo off-line de estimação de parâmetros, através do processamento de medidas de várias amostras (em lote ou seqüencialmente);

- A utilização de medidas de várias amostras possibilita o aumenta da redundância das medidas, que é um quesito de fundamental importância para estimação de parâmetros;

- Outra vantagem de proceder-se a estimação de parâmetros de maneira off-line é a possibilidade de pré selecionar as informações que serão processadas Ou seja, serão selecionadas apenas amostras de medidas isentas de EGs e de erros de topologia;

- Vários trabalhos têm mostrado que é significativa a influência de erros nos parâmetros shunt das linhas de transmissão no processo de EESEP (MINGUEZ; CONEJO, 2007; LEFEBVRE et al, 2004; LEFEBVRE et al, 2006), principalmente em sistemas de transmissão com grande circulação (absorção e compensação) de potência reativa. Este é o 
caso, por exemplo, do sistema de transmissão da Hydro-Québec Trans-Ènergy. Desta forma, é necessário o desenvolvimento de metodologias que possibilitem a depuração de parâmetros série e shunt de linhas de transmissão.

Os resultados de diversas simulações computacionais apresentadas no capítulo 5 comprovam a eficiência da metodologia proposta, pois, mesmo em condições de EPs múltiplos em ramos adjacentes, a mesma possibilitou significativas correções dos valores dos parâmetros.

Por último vale ressaltar que se comprovou a viabilidade prática da metodologia proposta através da aplicação da mesma para depuração dos valores dos parâmetros de dois subsistemas da Hydro-Québec TransÉnergie.

\subsection{Considerações finais e Trabalhos futuros}

$\mathrm{O}$ ambiente de desenvolvimento de ferramentas de apoio aos operadores dos SEP não está plenamente consolidado e o ambiente de desenvolvimento de estimadores mais robustos vem experimentando ampla evolução, mas pode e deve ser aperfeiçoado. Eis a razão de ter sido desenvolvida, neste trabalho, uma metodologia para depuração de parâmetros de linhas de transmissão, visando à obtenção de uma solução de estimação de estado mais confiável. Dessa forma, buscando um aprimoramento contínuo da metodologia proposta, pesquisas futuras serão desenvolvidas para possibilitar um melhoria da mesma. Nesse sentido, apresentam-se as seguintes sugestões para trabalhos futuros:

- Investigar a possibilidade de tornar mais robusta a Fase 1 da metodologia proposta, no sentido de permitir a detecção e identificação de ramos suspeitos, mesmo através de amostras de medidas portadoras de EGs. A idéia é aplicar o Índice de NãoDetecção de EGs (INDE ou UI do inglês, Undetectability Index), proposto em (BRETAS et al., 2009). As medidas com maiores UI são aquelas cujos erros são mais difíceis de serem detectados via teste dos resíduos normalizados e do índice $J(x)$. Isto porque uma grande parcela dos erros dessas medidas não aparece nos seus resíduos, isto é, os erros naquelas medidas são, de certa forma, "mascarados". Dessa forma, através do índice UI é possível qualificar as medidas de acordo com as suas características de "mascararem" os seus erros. Mais precisamente, o índice UI informa a proximidade de um ponto definido por um vetor unitário pertencente ao 
eixo de uma dada medida até a imagem da matriz Jacobiana do estimador por mínimos quadrados ponderados. As medidas com elevados $U I$ são as medidas que estão mais próximas da imagem da matriz Jacobiana. Face ao exposto, acreditamos que analisando os resíduos normalizados das medidas, juntamente com os seus índices $U I$, seja possível distinguir EGs de EPs;

- Pretendemos também estender a metodologia proposta para possibilitar a utilização não apenas de medidas convencionais, mas também de medidas fasoriais sincronizadas. 


\section{REFERÊNCIAS}

ABOYTES, F.; CORY, B. (1975). Identification of measurement, parameter and configuration errors in static state estimation, Proceedings of the IEEE Pica Conference, New Orleans. New York p.298-302.

ABUR, A.; EXPÓSITO, A.G. (2004). Power system state estimation: theory and implementation. Marcel \& Dekker Publishers, Nova York, EUA.

AYRES, M.; HALEY, P.H. (1986). Bad Data Groups in Power System State Estimation. IEEE Transaction on Power Systems, Vol. 1, $\mathrm{n}^{\circ}$. 3, pp.1-7, Agosto, DOI 10.1109/MPER.1986.4334946.

ALSAÇ, O.; VEMPATI, N.; STOTT, B.; MONTICELLI, A. (1998). Generalized State Estimation. IEEE Transactions on Power Systems, Vol.13, n.3, pp.1069-1075, August, DOI: $10.1109 / 59.709101$.

ALVARADO, F.; TINNEY, W.F. (1990). State estimation using augmented blocked matrices. IEEE Transactions on Power Systems, Vol.5, n 3, pp.911-921, August, DOI $10.1109 / 59.65921$.

ALLAM, M.F.; LAUGHTON, M.A. (1974). A General algorithm for estimating power system variables and network parameters. Proceedings of the IEEE Power Energy Society Summer Meeting \& Energy Resources Conference, Anaheim.

ALLEMONG, J.J.; RADU, L.; SASSON, A.M. (1982). A Fast Reliable State Estimator Algorithm for AEP's New Control Center, IEEE Transactions on Power Apparatus and Systems, Vol. PAS-101, pp. 933-944, April, DOI 10.1109/TPAS.1982.317159. 
ASCHMONEIT, F.C.; PETERSON, N.M.; ADRIAN, E.C. (1977). State estimation with equality constraints. Proceedings of the IEEE Pica Conference, Vol. 10, pp.427-430. Toronto.

BRETAS, N.G.; LONDON J.B.A.; ALBERTO, L.F.C.; , R.A.S. (2009) Geometrical approach on masked Gross errors for power systems state estimation. Proceedings of the IEEE Power Energy Society General Meeting. pp. 1-7, DOI:101109/PES.2009.5275941.

BRETAS, N.G. (1996). Network observability: Theory and algorithms based on triangular factorization and path graph concepts. IEE Proceedings, Generation, Transmission and Distribution, New York, Vol.143, nº ${ }^{\circ}$, pp.123-128, January, DOI 10.1049/IPGTD:19960169.

BENEDITO, R.A.S.; MOREIRA, E.M.; LONDON, J.B.A.; BRETAS, N.G.;(2008) Observability analysis based on path graph concepts and triangular factorization of the Jacobian matrix, Proceedings of the IEEE/PES Transmission and Distribution Conference and Exposition: Latin America, pp. 1 - 7, DOI:10.1109/TDC-LA.2008.4641699.

CASTILLO, M.R.M.; LONDON JR., J.B.A.; BRETAS, N.G. (2009a). Identification and Estimation of Power System Branch Parameter error. Proceedings of the IEEE Power \& Energy Society General Meeting, pp. 1-8, DOI: 10.1109/PES.2009.5275219.

CASTILLO, M.R.M.; LONDON JR., J.B.A.; BRETAS, N.G. (2009b). Network branch parameter validation based on a decoupled state/parameter estimator and historical data. Proceedings of the IEEE Bucharest PowerTech Conference, 2009.

CLEMENTS, K.A.; KRUMPHOLZ, G.R.; DAVIS, P.W. (1981). Power system state estimation residual analysis: an algorithm using network topology. IEEE Transactions on Power Apparatus and Systems, New York, Vol. PAS 100, n 4, pp.1779-1787, April, DOI: 10.1109/TPAS.1981.316517. 
EHRENSPERGER, J.G. (2004). Sistemas de medição fasorial sincronizada: análise do estado da arte e aplicações no monitoramento de sistemas de energia elétrica. Dissertação (Mestrado). Departamento de Engenharia Elétrica, Universidade Federal de Santa Catarina (UFSC), Florianópolis, Brasil.

DEBS A.(1974) Estimation of steady -state power system model parameters. IEEE Transactions on Power Systems, Vol. PAS-93, ${ }^{\circ}$ 5, pp. 1260-1268, September, DOI: 10.1109/TPAS.1974.293849.

DE ALMEIDA, M.C.; ASADA, E.N.; GARCIA, A.V.(2006) Effects of Load Imbalance and System Asymmetry on Three-phase state estimation Proceedings of the IEEE Power Energy Society General Meeting, D.O.I 10.1109/PES.20061709586

DE ALMEIDA, M.C.; ASADA, E.N.; GARCIA, A.V. (2008). Power system observability analysis based on gram matrix and minimum norm solution. IEEE Transactions on Power Systems, New York, Vol. 23, $\mathrm{n}^{\circ}$ 4, pp.1611-1618, November, DOI: 10.1109/PES.2006.1709586.

DE ALMEIDA, M.C.(2007) Tese de Doutorado: Estimação de Estado Generalizada Trifásica, Universidade Estadual de Campinas, Faculdade de Engenharia Elétrica e de Computação, Departamento de Sistemas de Energia Elétrica.

DO COUTTO FILHO, M.B.; LEITE DA SILVA, A.M.; FALCÃO, D.M. (1990). Bibliography on power system state estimation (1968-1989). IEEE Transactions on Power Systems, Vol. 5, n. 3, pp. 950-961, Agosto.

DO COUTTO FILHO, M.B. ; SOUZA, J.C.S. (2009). Forecasting-Aided State Estimation - Part I: Panorama. IEEE Transactions on Power Systems, Vol. 24, n . 4, pp. 1667-1677, Setembro, DOI: 10.1109/TPWRS.2009.2030295. 
DO COUTTO FILHO, M.B.D.; SOUZA, J.C.S.; OLIVEIRA, F.M.F.; SCHILLING, M.Th. (2001). Identifying critical measurements and sets for power system state estimation. Proceedings of IEEE Porto PowerTech, paper EDT2-147, pp. 1-6, Porto, Portugal, Setembro.

DO COUTTO FILHO, M;B.; STACCHINI DE SOUZA, J.C.; MEZA, E.B.M. (2008). Offline validation of power network branch parameters. IET Generation, Transmission \& Distribution, New York, Vol. 2, $\mathrm{n}^{\circ}$ 6, pp. 892-905, November, DOI:10.1049/ietgtd:20080143.

FLETCHER, D.L.; STADLIN, W.O. (1983). Transformer tap position estimation. IEEE Transactions on Power Apparatus and Systems, New York, v.PAS 102, n.11, p.3680-3686, November, DOI:10.1109/TPAS.1983.317732.

FREUND, R.S. (2006). Implantação da função de estimação de estado em um sistema de gerenciamento de energia. Dissertação (Mestrado) - Universidade Federal Fluminense, Rio de Janeiro, 2006.

GARCIA, A.; MONTICELlI, A.; ABREU, P. (1979). Fast Decoupled State Estimation and Bad Data processing. IEEE Transactions on Power Apparatus and Systems, Vol. PAS98, n 5, pp. 1645-1652, september, DOI:10.1109/TPAS.1979.319482.

GJELSVKI, A.; AAM, S.; HOLTEN, L. (1985). Hatchel's augmented matrix method: a rapid method improving numerical stability in power system static state estimation. IEEE Transactions on Power Apparatus and Systems, New York, Vol. PAS -104, $\mathrm{n}^{\circ}$ 11, pp. 2987-2993, November, DOI:10.1109/TPAS.1985.318939.

GU, J.W.; CLEMENTS, K.A. ; KRUMPHOLZ, G.R. ; DAVIS, P.W. (1983).The Solution of ill-conditioned power system state estimation problems via the method of Peters and Wilkinson. IEEE Transactions on Power Apparatus and Systems. Vol. PAS-102, pp. 34733480, DOI: 10.1109/TPAS.1983.317846. 
HANDSCHIN, E. et al. (1975). Bad data analysis for power systems state estimation. IEEE Transactions on Power Apparatus and Systems, New York, Vol. PAS 94, n² 2, pp. 329-337, March, DOI: 10.1109/TPAS.1975.52087.

HEATH, M. T.(1997) Nonlinear Equations - Lecture Notes to Accompany, Scientific Computing an Introductory Survey, Chapter 5.

HOLTEN, L.; GJELSVIK, A.; AAM, S. ; WU, F.F. ; LIU, W.H.E. (1988). Comparison of Different Methods for State Estimation. IEEE Transaction on Power Apparatus and Systems, Vol. 3, № 4, p.1798-1806, November, DOI: 10.1109/59.192998.

HORISBERGER, H.P.; RICHARD, J.C.; ROSSIER, C. (1976). A Fast decoupled static state estimator for electric power systems, IEEE Transactions on Power Apparatus and Systems, New York, Vol. PAS 95, $\mathrm{n}^{\circ}$ 1, p.208-215, January, DOI: 10.1109/TPAS.1976.32093.

JENNINGS A. (1985) "Matrix Computation for Engineers and Scientists". Wiley - Interscience publication, October.

KORRES, G. N. ; CONTAXIS, G.C. (1991a) A reduced model for bad data processing in state estimation. IEEE Transaction on Power Systems, New York, Vol. 6, n . 2, pp.550-557, May, DOI:10.1109/59.76697.

KORRES, G. N. ; CONTAXIS, G.C. (1991b) Identification and updating of minimally dependent sets of measurements in state estimation. IEEE Transaction on Power Systems, New York, Vol. 6, n. 3, pp.999-1005, August, DOI:10.1109/59.119239

KORRES, G.N.; CONTAXIS, G.C. (1994). A Tool for the evaluation and selection of state estimator measurement schemes. IEEE Transaction on Power Systems, New York, Vol.9, n², pp.1110-1116, May, DOI: 10.1109/59.317619 
KRUMPHOLZ, G.R.; CLEMENTS, K.A.; DAVIS, P.W. (1980). Power systems observability: a practical algorithm using network topology. IEEE Transactions on Power Apparatus and Systems, New York, Vol. PAS 99, $\mathrm{n}^{\circ} 4$, pp.1534-1542, July, DOI: 10.1109/TPAS.1980.319578.

KUSIC, G.L.; GARRISON, D.L. (2004). Measurement of transmission line parameters from SCADA data. Proceedings of the IEEE Power Systems Conference And Exposition, 2004. Vol.1, pp. 440-445, DOI:10.1109/PSCE.2004.1397479.

LEFEBVRE, S.; PREVOST, J. ; HORISBERGER, H.; LAMBERT, B (2004). Coping with Q-V solutions of the WLS state estimator induced by shunt-parameter errors, International Conference on Probabilistic Methods Applied to Power Systems, pp. 186-192, DOI: 10.1109/PMAPS.2004.241486.

LEFEBVRE, S.; PREVOST, J. ; HORISBERGER, H.; LAMBERT, B.(2006). On the Accuracy of state estimation. Proceedings of the IEEE Power Engineering Society General Meeting, DOI:10.1109/PES.2006.1709163.

LEFEBVRE, S.; PREVOST, J. ; RIZZI J.C.; YE, P. ; LAMBERT, B.; HORISBERGER, H.; (2008). Operational Experience with State Estimation at Hydro-Québec. Proceedings of the IEEE Power and Energy Society General Meeting - Conversion and Delivery Of Electrical Energy In The 21st Century, pp. 1-8, DOI : 10.1109/PES.2008.4596318.

LIU, W.H.E.; LIM, S.L. (1995). Parameter error identification and estimation in power system state estimation. IEEE Transactions on Power Systems, New York, Vol.10, $\mathrm{n}^{\circ} 1$, p.200-209, February, DOI:10.1109/59.373943.

LIU, W.H.E.; WU, F.F.; LUN, S.M. (1992). Estimation of parameter errors from measurement residuals in state estimation. IEEE Transactions on Power Systems, New York, Vol.7, n 1, pp.81-89, February, DOI:10.1109/59.141690. 
LONDON, J.B.A.JR.; ALBERTO, L.F.C; BRETAS, N.G. (2007). Analysis of measurement-set qualitative characteristics for state- estimation purposes. IET Generation, Transmission \& Distribution, New York, v.1, n.1, p.39-45.

LONDON, J.B.A. JR.; MILI, L.; BRETAS, N.G. (2004 a). An Observability analysis method for a combined parameter and state estimation of a power system. International Conference On Probabilistic Methods Applied To Power Systems, pp. 594-599.

LONDON, J.B.A. JR., PIERETI, S.A.R., , R.A.S. e BRETAS, N.G. (2009). Redundancy and Observability Analysis of Conventional and PMU Measurements. IEEE Transactions on Power Systems, Vol.24, $\mathrm{n}^{\circ}$ 3; pp. 1629- 1630, DOI: 10.1109/TPWRS.2009.2021195.

MERRILL, H.M.; SCHWEPPE, F.C. (1973). On-line system model error correction. Proceedings of IEEE Winter Power Meeting, pp. C73 106-2.

MEZA, E.B.M. et al. (2006a). Estimação de parâmetros de redes elétricas, X Simpósio de Especialistas em Planejamento da Operação e Expansão Elétrica, Vol. 1, pp. 1-8, Florianópolis.

MILI.; VAN CUTSEM, Th.; RIBBENS-PAVELLA, M. (1984). Hypothesis Testing Identification: A New Method for Bad Data Analysis in Power System State Estimation. IEEE Transactions on Power Apparatus and Systems, New York, Vol. 103, n ${ }^{\circ} 11$, pp. 3239-3252, November,DOI: 10.1109/TPAS.1984.318561.

MÍNGUEZ, R.; CONEJO, A.J. (2007). State Estimation sensitivity analysis. IEEE Transactions on Power Systems, New York, Vol.22, n 3, pp.1080-1091, August, DOI: 10.1109/TPWRS.2007.901488.

MONTICELLI, A. (1999). State Estimation in Electric Power Systems: A Generalized Approach. Kluwer Academic Publishers, Massachusetts, USA. 
MONTICELLI, A. (2000). Electric Power System State Estimation. Proceedings of the IEEE, Vol. 88, n 2, Fevereiro. (DOI: 10.1109/5.824004)

MONTICELLI, A.; WU, F.F. (1985a). Network observability: Identification of observable islands and measurement placement. IEEE Transaction on Power Apparatus and Systems, Vol. PAS-104, n . 5, pp. 1035-1041, May, DOI: 10.1109/TPAS.1985.323453.

MONTICELLI, A.; WU, F.F. (1985b). Network observability: Theory. IEEE Transaction on Power Apparatus and Systems, Vol. PAS-104, n. 5, pp. 1042-1048, May, DOI:10.1109/TPAS.1985.323454.

MONTICELLI, A.; WU, F.F. (1986). Observability analysis for orthogonal transformation based state estimation. IEEE Transactions on Power Systems, Vol.1, n. 1, pp. 201-206, February, DOI:10.1109/TPWRS.1986.4334870.

MONTICELLI, A.; GARCIA, A. (1990). Fast decoupled state estimators.IEEE Transaction on Power Systems, New York, Vol.5, $\mathrm{n}^{\circ} .2$, pp.556-564, May,DOI: 10.1049/ipqtd:19981785.

MONTICELLI, A.; WU, F.F. ; MURARI, C.A.F.(1985). A Hybrid state estimator: solving normal equations by orthogonal transformations. IEEE Transactions on Power Apparatus and Systems, New York, Vol.PER-5, $\mathrm{n}^{\circ}$ 12, pp.3460-3468, December, DOI:10.1109/MPER.1985.5528622.

MONTICELLI, A.; GARCIA, A. (1983). Reliable bad data processing for real -time State Estimation. IEEE Transactions on Power Apparatus and Systems, New York, Vol. PAS 102, n 5, pp.1126-1139, May, DOI:10.1109/TPAS.1983.318053. 
NUCERA, R.R.; GILLES, M.L. (1991). Observability analisys: A new topological algorithm. IEEE Transactions on Power Systems, Vol. 6, n 2, pp. 466 - 475, May, DOI: 10.1109/59.76688.

ORTEGA, J. M. AND RHEINBOLDT,(1970) Iterative Solution of Nonlinear Equations in Several Variables, Academic Press, New York.

PHADKE, A.G.; THORP, J.S.; KARIMI, K.J. (1986). State estimation with phasor measurements.Power Engineering Review,IEEE, New York, Vol. PER-6, n 2, pp.233-240, February, DOI: 10.1109/MPER.1986.5528179.

PHADKE, A.G. (1993). Synchronized Phasor Measurements in Power Systems. IEEE Computer Applications in Power, Vol. 6, $\mathrm{n}^{\circ}$ 2, pp. 10-15, Abril, DOI: 10.1109/67.207465.

PHADKE, A.G. (2002). Synchronized phasor measurements - a historical overview. Proceedings of IEEE Power Eng. Soc. Asia Pacific Transmission Distribution Conf. Exhib., Oct. 6-10, Vol. 1, pp. 476-479, October, DOI:10.1109/TDC.2002.1178427.

PRESS, W. H., TEUKOLSKY, S. A., VETTERLING, W. T., FLANNERY, B. P.( 1986) Numerical Recipes in Fortran 77 - The Art of Scientific Computing, Vol. 1, 2nd. edition. http://www.nr.com

PIERETI, S.A.R.; DELBEM, A.C.B.; LONDON, J.B.A.; BRETAS, N.G (2007). Tracking Network Topology Processor Using Node-depth Representation; Proceeding of the IEEE Power Tech, 2007, Lausanne DOI: 10.1109/PCT.2007.4538307, pp. $143-148$

QUINTANA, V.H.; SIMOES-COSTA, A.; MANDEL, A. (1982). Power system topological observability using a direct graph-theoretic approach. IEEE Transactions on Power Apparatus and Systems, New York, Vol.PAS 101, nº.3, pp.617-626, March, DOI: 10.1109/MPER.1982.5520319. 
REIG, A.; ALVAREZ, C. (1989). Influence of network parameter errors in state estimation results. Proceeding of the IASTED POWER HIGH TECH'89, Valencia. pp. 199-204.

SCHWEPPE, F.C.; WILDES, J. (1970). Power system static-state estimation, part I: exact model. IEEE Transactions on Power Apparatus and Systems, New York, Vol. PAS 89, $\mathrm{n}^{\circ} 1$, pp.120-125, January, DOI:10.1109/TPAS.1970.292678.

SCHWEPPE, F.C.; ROM, D.B. (1970). Power system static-state estimation, part II: approximate model. IEEE Transactions on Power Apparatus and Systems, New York, Vol.PAS 89, nº 1, pp.125-130, January, DOI:10.1109/TPAS.1970.292679.

SCHWEPPE, F.C. (1970). Power system static-state estimation, part III: Implementation, IEEE Transactions on Power Apparatus and Systems, New York, Vol.PAS 89, $\mathrm{n}^{\circ} 1$, pp.130-135, January, DOI: 10.1109/TPAS.1970.292680.

SCHWEPPE, F.C.; HANDSCHIN, E.J. (1974). Static State Estimation in Electric Power Systems. Proceeding of the IEEE, New York, Vol. 62, $\mathrm{n}^{\circ}$ 7, pp.972-982, DOI: 10.1109/PROC.1974.9549.

SILVA GOUVÊA, J.P.; SIMÕES COSTA, A.J.A. (1998). Estimador de estados ortogonal com restrições de igualdade. Controle \& Automação, Porto Alegre, Vol. 9, n 3, pp. 21312136, set./dez.

SIMÕES COSTA, A.; PIAZZA, T.S.; MANDEL, A. (1990). Qualitative methods to solve qualitative problems in power system state estimation. IEEE Transactions on Power Systems, Vol. 5, n³, pp. 941-949, August, DOI:10.1109/59.65924.

SIMÕES COSTA, A.; QUINTANA, V.H. (1981). A Robust Numerical Technique for Power System State Estimation. IEEE Transactions on Power Apparatus and Systems, Vol. PAS-100, n² 2, pp. 691-698, February, DOI: 10.1109/TPAS.1981.316920. 
SLUTSKER, I.W.; MOKHTARI, S.; CLEMENTS, K.A. (1996). Real time recursive parameter estimation in energy management systems. IEEE Transactions on Power Systems $_{2}$ New York, Vol.11, n.3, pp.1393-1399, August, DOI: 10.1109/59.535680.

SMITH, R.A. (1985). Transformer tap estimation at Florida Power Corporation. IEEE Transactions on Power Apparatus and Systems, New York, Vol.PAS 104, n 12, pp.34423445, December, DOI: 10.1109/TPAS.1985.318893.

STUART, T.A. ; HERCZET, C. J. (1973). A Sensitivity analysis of weighted least squares state estimation for power systems. IEEE Transactions on Power Apparatus and Systems, New York, Vol. PAS 92, n.5, pp.1696-1701, Sept./Oct, DOI: 10.1109/TPAS.1973.293718.

STACCHINI DE SOUZA, J.C.; DO COUTTO FILHO M.B. ; MEZA E.B.M. (2009) Treatment of multiple network parameter errors through a genetic-based algorithm, Electric Power Systems Research, Vol. 79, pp. 1546-1552.

THORP, J.S.; PHADKE, A.G.; KARIMI, K.J. (1985). Real time voltage-phasor measurements for static state estimation. IEEE, Power Engineering Review, Vol. PER-5, $\mathrm{n}^{\circ} .11$, p. 32-33, DOI: 10.1109/MPER.1985.5528364.

VAN CUTSEM, T.; QUINTANA, V. H. (1988). Network parameter estimation using online data with application to transformer tap position estimation. Proceedings of the IEEE: generation, transmission and distribution, New York, Vol.135, $\mathrm{n}^{\circ} 1$, p.31-40, January.

VUONG, H. M.; LEFEBVRE, S.(2002). Detection and Estimation of Topological and Parameter errors from real-time measurements. Power Engineering Society Summer Meeting, IEEE, Vol. 3, pp. 1565-1569, August, DOI: 10.1109/PESS.2002.1043653. 
ZARCO, P.; EXPÓSITO, A.G. (2000). Power System Parameter Estimation: A Survey, IEEE Transactions on Power Systems, Vol.15, $\mathrm{n}^{\circ}, 1$, pp.216-222, February,DOI: $10.1109 / 59.852124$.

ZARCO, P. EXPOSITO, A. G. (1997). Estimación de Parámetros en redes Eléctricas: Problemática y Soluciones Adoptadas. 5th. Jornadas Hispano-Lusas de Ingeniería Eléctrica, pp. 911-918, Salamanca, España, Julio.

ZARCO, P.; EXPOSITO, A. G. (1996). Off-line determination of network parameters in state estimation, Proceedings $12^{\text {th }}$ Power System Computation Conference, pp. 12071213,Dresden, Germany, August.

ZIVANOVIC, R.; CAIRNS, C. (1996).Implementation of PMU technology in state estimation: an overview. Proceeding of the IEEE $4^{\text {th }}$ AFRICON, Vol. 2, pp. 1006-1011, Setembro, DOI: 10.1109/AFRCON.1996.563034.

ZHU, J. ; ABUR, A. (2006). Identification of network parameter errors, IEEE Transactions on Power Systems, Vol. 21, $\mathrm{n}^{\circ}$ 2, pp.586-592, February , DOI:10.1109/TPWRS.2006.873419.

ZHU, J. ; ABUR, A. (2009). Identification of network parameter errors using phasor Measurements, Proceeding of the IEEE Power and Energy Society General Meeting, pp. 15, Calgary,Canada, July, DOI: 10.1109/PES.2009.5275490.

ZHU, J. ; ABUR, A. (2010) Improvements in Network Parameter Error identification via Synchronized Phasors, IEEE Transactions on Power Systems, Vol. 25, $\mathrm{n}^{\circ}$ 1, pp.44-50, February , DOI:10.1109/TPWRS.2009.2030274.

ZHU, J. ; ABUR, A. (2007). Effect of Phasor Measurements on the Choice of Reference Bus for State Estimation. Proceedings of the Power Engineering Society General Meeting 2007, pp. 1-5, DOI:10.1109/PES.2007.386175. 
ZHOU, M.; CENTENO, V. A; THORP, J. S.; PHADKE, A.G. (2006). An Alternative for Including Phasor Measurements in State Estimators. IEEE Transactions on Power Systems, Vol. 21, $\mathrm{n}^{\circ}$ 4, pp.1930-1937, Novembro, DOI: 10.1109/TPWRS.2006.881112.

ZHOU, M.; CENTENO, V.A.; PHADKE, A.G.; HU, Y.; NOVOSEL, D.; VOLSKIS, H.A.R. (2008). Proceedings of Third International Conference on Electric Utilily Deregulation and Restructuring and Power Technologies - DRPT 2008, p. 2862-2867, Abril, DOI: 10.1109/DRPT.2008.4523897. 


\section{Apêndice A}

\section{Publicações Originadas desta Pesquisa}

\subsection{Artigo publicado em revista internacional}

a) Título: "Off-line Detection, Identification and Correction of Branch Parameter Errors Based on Several Measurement Snapshots”,

Autores: Madeleine R. Medrano Castillo, João Bosco London Junior e Newton Geraldo Bretas, Serge Lefebvre, Prévost, B. Lambert.

Nome do Periódico: IEEE Transactions on Power Systems, Vol. PP, n 99,pp. 1-9, DOI:10.1109/TPWRS.2010.2061876.

\subsection{Artigos completos publicados em anais de congressos internacionais}

a) Título: "Off-line Detection, Identification and Estimation of Power System Branch Parameter Error",

Autores: Madeleine R. Medrano Castillo, João Bosco London Junior e Newton Geraldo Bretas.

Nome e lugar do Evento: IEEE, PES General Meeting, 25 - 29 Jul. 2010, Minneapolis, Minesota, USA, DOI: 10.1109/PES.2010.5589552.

b) Título: "Identification and Estimation of Power System Branch Parameter Error",

Autores: Madeleine R. Medrano Castillo, João Bosco London Junior e Newton Geraldo Bretas. 
Nome e lugar do Evento: IEEE, PES General Meeting, 26 - 30 Jul. 2009, Calgary, Alberta, Canada, DOI: 10.1109/PES.2009.5275219.

c) Título: "Network Branch Parameter Validation Based On a Decoupled State /Parameter Estimator and Historical Data",

Autores: Madeleine R. Medrano Castillo, João Bosco London Junior e Newton Geraldo Bretas.

Nome e lugar do Evento: IEEE, PES PowerTech, 28 June - 2 July 2009, Bucharest, Romania.

d) Título: "An Approach to Power System Branch Parameter Estimation",

Autores: Madeleine R. Medrano Castillo, João Bosco London Junior e Newton Geraldo Bretas.

Nome e lugar do Evento: Anais do IEEE Electrical Power Conference (CD artigo $\quad \mathrm{N}^{\circ}$ 1374_CR, 2008) Vancouver -Canada, 2008, DOI:10.1109/EPC.2008.4763381.

e) Título: "Electrical Network Branch Parameters Validation for Effects of State Estimation",

Autores: Madeleine R. Medrano Castillo, João Bosco London Junior e Newton Geraldo Bretas.

Nome e lugar do Evento: Anais 2008 IEEE PES Transmission and Distribution Conference and Exposition, v. 1. p.1-6, Bogotá-Colombia, 2008

f) Título: "Estimación de Estado y Parametros de Lineas de Transmisión", Autores: Madeleine R. Medrano Castillo, João Bosco London Junior e Newton Geraldo Bretas. 
Nome e lugar do Evento: Anais XVII CONIMERA -, 2007, Lima- Perú pag. 190197.

g) Título: “An approach for transmission line parameters and state estimation",

Autores: Madeleine R. Medrano Castillo, João Bosco London Junior e Newton Geraldo Bretas.

Nome e lugar do Evento: Proceedings of the Sixth World Energy System Conference (CD - número do paper:A2.5.pdf, 7 PÁGINAS (78-84)), 2006.. , 2006. p.78- 84

\subsection{Artigos completos publicados em anais de congressos nacionais}

a) Título: "Programa computacional para avaliação da infra-estrutura disponível, para efeito de estimação de estado em sistemas elétricos de potência”,

Autores: Madeleine R. Medrano Castillo, João Bosco London Junior e Newton Geraldo Bretas.

Nome e lugar do Evento: IX EDAO ENCONTRO PARA DEBATES DE ASSUNTOS DE OPERAÇÃO, 2007, Rio Quente, 2006, Goias. (CD - artigo se36.pdf - 12 páginas), 2007.

b) Título: “An Approach for Transmission Line Parameters and State Estimation”,

Autores: Madeleine R. Medrano Castillo, João Bosco London Junior e Newton Geraldo Bretas.

Nome e lugar do Evento: Anais XVI- CBA CONGRESSO BRASILEIRO DE AUTOMATICA (CD - número do paper:379, 6 páginas), 2006.

a) Título: "Metodologia off-line para Detecção, Identificação e Correção de Parâmetros de Linhas de Transmissão", 
Autores: Madeleine R. Medrano Castillo, João Bosco London Junior e Newton Geraldo Bretas.

Nome e lugar do Evento: CBA, XVIII Congresso Brasileiro de Automática, 12 16 Setembro. 2010, Bonito, Mato Grosso do Sul

\subsection{Artigo aceito para apresentação em congresso Nacional}

a) Título: "Metodologia off-line para validação dos parâmetros de linhas de transmissão do sistema elétrico de potência da empresa canadense Hydro-Quebec",

Autores: Madeleine R. Medrano Castillo, João Bosco London Junior e Newton Geraldo Bretas, Serge Lefebvre, Prévost, B. Lambert.

Nome e lugar do Evento: XI EDAO ENCONTRO PARA DEBATES DE ASSUNTOS DE OPERAÇÃO, 22 a 26 de novembro de 2010, Florianopolis.

\subsection{Artigo submetido para congresso Internacional}

a) Título: "Validation of an off-line approach for transmission line parameters estimation on two Hydro-Québec subsystems",

Autores: Madeleine R. Medrano Castillo, João Bosco London Junior e Newton Geraldo Bretas, Serge Lefebvre, Prévost, B. Lambert.

Nome e lugar do Evento: $17^{\text {th }}$ Power Systems Computation Conference, August 22-26, PSCC 2011,Stockholm. 


\section{Apêndice B}

\section{Obtenção dos dados para simulação}

Nos testes com os sistemas do IEEE apresentados no capítulo 5, os valores das medidas utilizadas foram obtidas a partir de um programa computacional desenvolvido em MATLAB (6.0), que adiciona, aos valores obtidos por um programa de fluxo de carga (ANAREDE), erros aleatórios de média zero e desvio padrão calculado através da equação (B.2).

Para justificar a utilização da equação (B.2), neste apêndice discutir-se-ão as incertezas das medidas utilizadas no processo de EESEP.

\section{B.1 Incertezas nas Medidas}

Como visto nas seções anteriores, o processo de EESEP depende principalmente do grau de exatidão dos dados, que são considerados no processo, como:

- A informação da topologia da rede;

- Os parâmetros elétricos;

- A localização e tipo das medições, quais sejam: telemedidas - provenientes do sistema SCADA; pseudomedidas; dados históricos e Medidas Virtuais.

Cada um desses itens possui seu grau de dificuldade específico, sendo que os dois primeiros dependem de um bom cadastro de informações e de ações de manutenção continuada, o que nem sempre acontece.

Os tipos de telemedidas, usualmente considerados no processo de EESEP são os seguintes:

- Fluxos de potência ativa e reativa em linhas de transmissão, ramais, transformadores, capacitores série, geradores e cargas;

- Fluxos de potência reativa em compensadores síncronos, compensadores estáticos, reatores e bancos de capacitores;

- Injeção de potência ativa e reativa nas barras;

- Módulo e ângulo de fase de tensão; 
- Tap de transformador;

Esses dados telemedidos são portadores de erros inerentes ao sistema de medição, que vão desde o transformador de corrente (TC), ou de potencial (TP), até a apresentação na tela do SCADA. Assim, se a quantidade e localização das medidas preencherem adequadamente o requisito de observabilidade e redundância, a EE é realizada com a ponderação de cada uma das medidas disponibilizadas pelo sistema de medição.

Considera-se que os erros de medição seguem uma distribuição gaussiana e apresentam matriz de covariâncias $\mathrm{R}$ diagonal. Assim, os medidores que produzem variâncias $\left(\sigma^{2}\right)$ menores apresentam menores desvios e possuem maior grau de precisão, o que é retratado na matriz $\mathrm{R}$, dando maior peso às medidas mais precisas e menor peso, em caso contrário.

Os erros de medição podem ser classificados em um dos seguintes grupos:

- Erro extremo:I medida -valor exato $>20 \sigma$

- Erro grosseiro: I medida - valor exato $\mid=$ de $3 \sigma$ a $20 \sigma$

- Ruído normal: | medida - valor exato $\mid<3 \sigma$.

Os erros pertencentes ao primeiro grupo são filtrados, mediante uma pré-análise dos dados, isto é, antes do processo de EESEP propriamente dito (etapa de pré-filtragem das medidas). Entretanto, se um desses erros não for filtrado, naquela pré-análise, o processo de estimação de estado fará essa filtragem, desde que o nível de redundância seja adequado.

Os erros do segundo grupo são perigosos, em razão de poderem conduzir a resultados não confiáveis. Por este motivo, estes erros são eliminados pela etapa de análise de erros não gaussianos do EE.

Dentre os fatores listados em (ZARCO; EXPOSITO, 1997), os mais relevantes que devem ser levados em consideração para aferir a exatidão do processo, são: os transformadores de corrente e de potencial; e os dispositivos eletrônicos inteligentes (IEDs), representados pelos Medidores Digitais de Grandezas Elétricas (MDGEs).

Além dos TCs e TPs, diversos outros dispositivos e equipamentos influenciam também na precisão do sistema de medição, tais como:

- A calibração dos transdutores analógico/digital;

- A precisão da unidade terminal remota e do protocolo de enlace entre a subestação e o frontal de comunicações; 
- O fator térmico e a classe de precisão dos transformadores de medição;

- O desequilíbrio entre fases, etc.

São fatores determinantes na precisão:

- A classe de precisão dos equipamentos de medição, que leva em consideração a relação do erro máximo que uma medida contenha, em porcentagem do fundo de escala;

- A linearidade, ou seja, o erro relativo percentual, quanto ao valor de fundo de escala, com o equipamento perfeitamente calibrado;

- A condição de repetição, ou a diferença com relação a fatores dinâmicos da medição e,

- O desvio térmico, que é a consideração da influência de fatores ambientais, e outras influências, como freqüência da rede e outros fatores que possam interferir sobre o equipamento de medição.

O valor do desvio padrão dos erros das medidas, $\sigma_{m}$, considerado para as tele- medidas é geralmente definidos por tipo de medida, na fase inicial de implantação do Estimador de Estado. Nas publicações a respeito do assunto, existem diversas propostas para cálculo do desvio padrão dos erros das medidas (ZARCO;EXPOSITO, 1997).

A seguir apresentam-se relacionadas diferentes proposições para definição $\operatorname{dos} \sigma_{m}$, nos quatro grandes grupos a seguir definidos:

\section{1. $\sigma$ é um valor constante:}

a) Em (ABUR, 1990); (ABUR; ÇELIK, 1991); (ÇELIK; ABUR, 1992), propõe-se:

- Para medidas de injeção: $\sigma=0.01$ p.u.

- Para medidas de fluxo: $\sigma=0.008$ p.u.

- Para medidas de tensão $\sigma=0.004 p . u$. 
b) Em (BALDICK et. al., 1996); (CLEMENTS et. al., 1973) ; (CLEMENTS et. al., 1974), propõe-se:

- Para medidas de potência: $\sigma=1 M W / M V A . R$

- Para medidas de tensão: $\sigma=0.01$ p.u.

c) Em (HANDSCHIN et. al., 1975), propõe-se:

- Para medidas de potência: $\sigma=0.02$ p.u. (sobre uma base de 100 MVA)

- Para medidas de tensão: $\sigma=0.002 p \cdot u$.

d) Em (HANDSCHIN et. al., 1995), propõe-se:

- Para medidas de potência: $\sigma=1 M W / M V A . R$

- Para medidas de tensão: $\sigma=0.005$ p.u.

e) Em (MILI L. et. al., 1984; MILI et. al., 1985), propõe-se:

- Para medidas de potência: $\sigma=1.5 M W / M V A R$. em $132 \mathrm{KV}$ e $\sigma=0.8 M W / M V A R$. em $33 \mathrm{KV}$

- Para medidas de tensão: $\sigma=0.005$ p.u.

- Para medidas de injeção zero: $\sigma=0.2 M W / M V A R$.

f) Em (VAN CUTSEM; QUINTANA, 1988), propõe-se:

- Para medidas de potência: $\sigma=0.5 \div 1.1 M W / M V A R$. em $70 \mathrm{KV}$ e $\sigma=1.2 \div 5.5 M W / M V A R$. em tensões superiores.

- Para medidas de tensão: $\sigma=0.005$ p.u.

- Para medidas de injeção zero: $\sigma=0.3 M W / M V A R$. em $70 \mathrm{KV}$ e $\sigma=0.5 M W / M V A R$. em tensões superiores.

\section{2. $\sigma$ é função do valor medido:}

a) $\mathrm{Em}$

(ALLAM; LAUGHTON, 1974; MILI et. al., 1991), propõe-se:

- Para todas as medidas, $\sigma=1 \%$ do valor medido 
b) Em (LIU et. al, 1992), propõe-se:

- Para todas as medidas $\sigma \leq 2 \%$ do valor medido

c) Em (LIU; LIM, 1995), propõe-se:

- Para todas as medidas, $\sigma \leq 3 \%$ do valor medido

d) Em (MASIELLO; SCHWEPPE, 1971), propõe-se:

- Para todas as medidas $\sigma=2 \%$ do valor medido.

e) Em (RUIZ ; GOMEZ, 1992), propõe-se:

- Para todas as medidas $\sigma=0.01 \%$ do valor medido

\section{3. $\sigma$ é função do fundo de escala:}

a) Em (IRVING, et al., 1978), propõe-se:

- Para todas as medidas, o ruído gaussiano obtido de um gerador de números aleatórios é multiplicado por uma porcentagem, sem especificar, do fundo de escala do medidor.

b) Em (LARSONR, et. al., 1970), propõe-se:

- Para medidas de potencia: $\sigma=0.5 \%$ do fundo de escala, sendo este $1000 \mathrm{MW}$

- Para pseudo-medidas: $\sigma=10 \div 40 \%$ do fundo de escala.

c) Em (ZARCO, 2004), o valor do desvio padrão considerado foi:

- Para medidas de potência: $\sigma=0.1^{*} \gamma^{*} F E$ sendo FE o fundo de escala e $\gamma$ é a classe de precisão do dispositivo de medida, considera-se classe $\gamma=0.01$

- Na tabela A.1 apresenta-se, os valores considerados de FE's para medidores de Injeção e Fluxo de Potência para o sistema de 14 barras do IEEE (utilizou-se a base de 100 MVA)

Tabela B.1 FE de medidas potência do sistema 14 barras do IEEE. 


\begin{tabular}{|l|l|l|l|l|l|l|}
\hline $\begin{array}{l}\text { FE de medidas de injeção de } \\
\text { potência }\end{array}$ & \multicolumn{5}{l}{ FE de medidas de fluxo de potência } \\
\hline Barra & $\begin{array}{l}\text { Injeção } \\
\text { potência } \\
\text { ativa }\end{array}$ & $\begin{array}{l}\text { Injeção } \\
\text { potência } \\
\text { reativa }\end{array}$ & $\begin{array}{l}\text { Barra } \\
\text { inicial }\end{array}$ & $\begin{array}{l}\text { Barra } \\
\text { final }\end{array}$ & $\begin{array}{l}\text { Fluxo } \\
\text { potência } \\
\text { ativo }\end{array}$ & $\begin{array}{l}\text { Fluxo } \\
\text { potência } \\
\text { reativo }\end{array}$ \\
\hline 1 & 2.80 & 0.20 & 1 & 2 & 2.00 & 0.35 \\
\hline 2 & 0.70 & 0.65 & 2 & 3 & 1.00 & 0.20 \\
\hline 3 & 1.15 & 0.40 & 2 & 4 & 1.00 & 0.20 \\
\hline 4 & 0.70 & 0.95 & 1 & 5 & 1.00 & 0.20 \\
\hline 5 & 0.25 & 0.40 & 2 & 5 & 0.65 & 0.20 \\
\hline 6 & 0.60 & 0.65 & 3 & 4 & 0.65 & 0.20 \\
\hline 7 & 0.10 & 0.20 & 4 & 5 & 1.00 & 0.35 \\
\hline 8 & 0.10 & 0.40 & 5 & 6 & 0.65 & 0.35 \\
\hline 9 & 0.60 & 0.20 & 4 & 7 & 0.65 & 0.35 \\
\hline 10 & 0.25 & 0.10 & 7 & 8 & 0.20 & 0.35 \\
\hline 11 & 0.10 & 0.10 & 4 & 9 & 0.65 & 0.20 \\
\hline 12 & 0.10 & 0.10 & 7 & 9 & 0.65 & 0.20 \\
\hline 13 & 0.25 & 0.20 & 9 & 10 & 0.20 & 0.10 \\
\hline 14 & 0.25 & 0.20 & 6 & 11 & 0.25 & 0.20 \\
\hline & & & 6 & 12 & 0.20 & 0.10 \\
\hline & & & 6 & 13 & 0.25 & 0.20 \\
\hline & & & 9 & 14 & 0.20 & 0.10 \\
\hline & & & 10 & 11 & 0.20 & 0.10 \\
\hline & & & 12 & 13 & 0.20 & 0.10 \\
\hline & & & 13 & 14 & 0.20 & 0.10 \\
\hline
\end{tabular}

- Considerou-se $\mathrm{FE}=1$ para medidores de magnitude de tensão.

- Os fundos de escala, considerados para cada dispositivo de medida dependem do máximo valor esperado em cada ponto de medida. Pelo fato de serem distintos, são agrupados em torno de vários valores, com o objetivo de não haver muita diversidade de valores.

\section{4. $\sigma$ é função do valor medido e do fundo de escala:}

a) Em (ALLEMONG et. al., 1982), propõe-se:

Sendo:

$$
\sigma=0.0067 * V M+0.00163 * F E
$$

VM o valor medido.

FE o fundo de escala:

b) Em (CLEWER et. al., 1988), propõe-se: 


$$
\sigma=0.0015^{*} V M * \alpha+0.003 * F E^{*} \alpha
$$

sendo $\alpha$ um numero aleatório de média zero e desvio padrão 1.0 e os valores do fundo de escala:

- Para medidas de potencia:

- Para geradores: 1.2 p.u. para potência ativa e 0.2 p.u. para reativa.

- Para cargas: 0.2 p.u. para potência ativa e 0.1 p.u. para reativa.

- Para medidas de tensão: 1.5 p.u.

c) $\quad$ Em (DEBS ; LITZENBERGER, 1975), propõe-se:

- Para medidas de potência: $\sigma^{2}=(0.006 * V M)^{2}+\left(0.005^{*} F E\right)^{2}$

- Para medidas de tensão: $\sigma=0.005^{*} F E$. Não foi especificado o FE.

d) Em (DOPAZO et. al., 1971), propõe-se: Para as medidas de fluxo de potência

sendo:

$$
\sigma=\alpha *(0.02 * V M+0.0035 * F E)
$$

$|\alpha| \leq 1$

$F E=2000 M V A$

e) $\quad$ Em (FALCÃO; DE ASSIS 1988), propõe-se:

- Para medidas de potência: $\frac{1}{3}(0.005 * F E+0.02 * V M)$.

- Para medidas de tensão: $\sigma=0.001 * V M$

Não foi especificado o FE.

f) $\quad$ Em (LO et. al., 1983), propõe-se:

$$
\sigma=0.012 * V M+0.0035^{*} F E
$$

sendo $F E=20$ p.u.

g) Em (VAN SLYCK ; ALLEMONG, 1988), propõe-se:

$$
3 * \sigma=\alpha * V M+\beta * F E
$$

sendo:

- Para medidas de potência: $\alpha=0.02, \beta=0.0052$ e VM e FE são valores em MVA. 
- Para medidas de tensão: $\alpha=0.005, \quad \beta=0.0026, \quad V M=1.0$ p.u., $F E=1.5$ p.u.

- Para medidas de injeção zero: $\sigma$ é 20 vezes superior ao $\sigma$ típico de medidas de injeção reais.

h) Em (BELEZ; MACIEL, 1994), propõe-se:

$$
\sigma=P t e^{*}|Z|+A D e^{*}(F S)
$$

sendo:

- $|Z|$ - valor da medida (p.u) em módulo. Esta medida pode ser de injeção ou fluxo.

- Pte- erros nos transformadores de medida (tipicamente 2\%)

- $\mathrm{ADe}$ - erro devido a transdutores de potência ativa e reativa $(0.25 \%)$, e conversores $\mathrm{A} / \mathrm{D}(0.1 \%)$

- FS - Fundo de escala para transdutores e conversores (p.u)

Em relação às medidas de tensão, a expressão é igual, sendo no entanto neste caso:

- $|Z|$ - valor medido para a amplitude de tensão (p.u).

- Pte- erro nos transformadores de tensão (tipicamente $0.3 \%$ )

- $\mathrm{ADe}$ - erro devido a transdutores $(0.25 \%)$ e a conversores $\mathrm{A} / \mathrm{D}(0.05 \%)$

Resumindo, as expressões utilizadas são:

$$
\begin{aligned}
& \sigma_{i}=0.02 *|Z|+0.0035^{*}(F S), \text { para Pi, Qi, Pij, Qij } \\
& \sigma_{i}=0.003 * V+0.003 *(F S), \text { para } \mathrm{Vi}
\end{aligned}
$$

Evidentemente que nem todos os erros de medida (w) são independentes entre si, o que implicaria considerar uma matriz covariância $(\mathrm{R})$ com elementos não nulos fora da diagonal principal. A experiência tem, porém demonstrado que é aceitável considerar os erros de medida como independentes, o que permite considerar a matriz $\mathrm{R}$ com uma matriz diagonal.

Em relação às pseudomedidas, os valores de $\sigma_{i}$ dependem da precisão dos reguladores de tensão, dos modelos adotados para as cargas, previsões utilizadas, etc, podendo assumir portanto valores bastante superiores. 
Segundo (ZARCO;EXPOSITO, 1997) nos medidores industriais existe uma similar disparidade, com respeito à precisão do medidor. Dependendo do fabricante e do modelo, tal disparidade é proporcional à medida e ao fundo de escala, ou à soma desses fatores, mas nunca é constante.

Em (FREUND, 2006), os valores das medidas utilizadas nos testes foram obtidos por simulação, através da adição de incertezas aos resultados de um fluxo de potência. Assim, cada medida $\mathrm{Z}_{\mathrm{m}}$ será simulada, adicionando-se-lhe ao valor obtido pelo fluxo de carga, o produto definido pelas características dos medidores $\left(3 \sigma_{Z}\right)$ e um número aleatório com distribuição normal $(\alpha)$.

$$
\left\{\begin{array}{l}
Z_{m}=|Z|+\left(3 \sigma_{Z}\right) \alpha \\
\left(3 \sigma_{Z}\right)=p r *|Z|+f e
\end{array}\right.
$$

onde:

$\sigma_{Z}=$ Desvio padrão associado à medida

$|Z|$ = Valor absoluto da medida verdadeira, em determinado instante de tempo

$p r=$ Precisão do medidor

$f e=$ Fundo de Escala

$\alpha$ = Variável aleatória $\mathrm{N}(0,1)$

A tabela B.2 mostra os valores utilizados para os parâmetros $p r$ (precisão do medidor) e $f e$ (fundo de escala) em relação ao tipo de medidor.

Tabela B.2 - Parâmetros dos Medidores

\begin{tabular}{|l|c|c|}
\hline \multirow{2}{*}{ Tipo de Medidor } & \multicolumn{2}{|c|}{ Parâmetros } \\
\cline { 2 - 3 } & PR & fe \\
\hline Fluxo de potência & 0,02 & 0,035 \\
\hline Injeção de potência & 0,01 & 0,035 \\
\hline Magnitude de tensão & 0,01 & 0,000 \\
\hline
\end{tabular}

\section{5. $\sigma$ adotado neste trabalho:}

Tomando por base as diferentes proposições para o cálculo do desvio padrão das medidas, optou-se, neste trabalho, a utilização de precisão e do fundo de escala do medidor. 
Desenvolveu-se então um programa "computacional" em Matlab, para gerar conjuntos de medidas utilizadas para testar o estimador desacoplado de estado e parâmetros. A idéia é aquela alusiva às do sistema SCADA. Para isso, o programa adiciona, aos valores exatos, determinados por um programa de fluxo de carga (ANAREDE), erros aleatórios de média zero e um determinado desvio padrão. O desvio padrão, para cada medida, é determinado através da equação (B.2).

$$
\left\{\begin{array}{l}
Z_{m}=|Z|+\left(3 \sigma_{Z}\right) \alpha \\
\left(3 \sigma_{Z}\right)=p r * f e
\end{array}\right.
$$

onde:

$\sigma_{Z}=$ Desvio padrão associado à medida

$|Z|$ = modulo do vetor verdadeiro da medida $\mathrm{z}$, em determinado instante de tempo (obtido a partir do cálculo de fluxo de carga).

$p r=$ Precisão do medidor $=0.02$

$f e=$ Fundo de Escala

$\alpha$ = Variável aleatória $\mathrm{N}(0,1)$

Para gerar as medidas utilizadas nos testes com os sistemas de 14, 30 e 57 barras do IEEE.

Foram utilizados $\mathrm{FE}=1.1$ para medidores de magnitude de tensão;

A tabela B.3. ilustra os FEs utilizados para medidores de potência (fluxo e injeções), que dependem do valor verdadeiro de cada medida, isto é, obtido pelo fluxo de carga.

Tabela B.3 Fundo de escala para medidores de potência do sistema 14 barras do IEEE.

\begin{tabular}{|l|c|}
\hline & $\begin{array}{l}\text { FE de medidas de } \\
\text { Injeção e Fluxo }\end{array}$ \\
\hline $2.0 \leq|Z| \leq 2.5$ & 2.5 \\
\hline $1.0 \leq|Z| \leq 2.0$ & 2.0 \\
\hline $0.65 \leq|Z| \leq 1.0$ & 1.00 \\
\hline $0.35 \leq|Z| \leq 0.65$ & 0.65 \\
\hline $0.2 \leq|Z| \leq 0.35$ & 0.35 \\
\hline $0.1 \leq|Z| \leq 0.2$ & 0.2 \\
\hline
\end{tabular}




\begin{tabular}{|l|c|}
\hline $0.05 \leq|Z| \leq 0.1$ & 0.1 \\
\hline $0 \leq|Z| \leq 0.05$ & 0.05 \\
\hline
\end{tabular}

Na tabela B.4 apresenta-se, os valores considerados de FE's para medidores de Injeção e Fluxo de Potência para o sistema de 30 barras do IEEE (utilizou-se a base de 100 MVA)

Tabela B.4 FE para medidores de injeção de potência do sistema 30 barras do IEEE.

\begin{tabular}{|l|c|}
\hline & $\begin{array}{l}\text { FE de medidas de } \\
\text { Injeção e Fluxo }\end{array}$ \\
\hline $2.0 \leq|Z| \leq 2.8$ & 2.80 \\
\hline $1.8 \leq|Z| \leq 2.0$ & 2.00 \\
\hline $1.0 \leq|Z| \leq 1.8$ & 1.80 \\
\hline $0.5 \leq|Z| \leq 1.0$ & 1.00 \\
\hline $0.2 \leq|Z| \leq 0.5$ & 0.5 \\
\hline $0.1 \leq|Z| \leq 0.2$ & 0.2 \\
\hline $0 \leq|Z| \leq 0.1$ & 0.1 \\
\hline
\end{tabular}

Na tabela B.5 apresenta-se, os valores considerados de FE's para medidores de Injeção e Fluxo de Potência para o sistema de 57 barras do IEEE (utilizou-se a base de 100 MVA)

Tabela B.5 FE de medidas de injeção de potência do sistema 57 barras do IEEE.

\begin{tabular}{|l|c|}
\hline & $\begin{array}{l}\text { FE de medidas de } \\
\text { Injeção e Fluxo }\end{array}$ \\
\hline $2.0 \leq|Z| \leq 2.8$ & 2.80 \\
\hline $1.8 \leq|Z| \leq 2.0$ & 2.00 \\
\hline $1.0 \leq|Z| \leq 1.8$ & 1.80 \\
\hline $0.5 \leq|Z| \leq 1.0$ & 1.00 \\
\hline $0.2 \leq|Z| \leq 0.5$ & 0.5 \\
\hline $0.1 \leq|Z| \leq 0.2$ & 0.2 \\
\hline $0 \leq|Z| \leq 0.1$ & 0.1 \\
\hline
\end{tabular}




\section{Apêndice C}

\section{Métodos para solução de sistemas de equações não lineares}

\section{C.1 Introdução}

A resolução de um sistema de equações não lineares é uma tarefa necessária durante a resolução de problemas das mais diversas áreas(Fisica, Engenharia, Economia e outras Ciencias). Para tanto, são utilizados métodos iterativos não lineares.

O método iterativo não linear, mais conhecido é o método de Newton, o qual possui convergência local quadrática (ORTEGA; RHEINBOLDT, 1970).

$\mathrm{Na}$ solução de sistemas de equações algébricas não-lineares pelo método de Newton,admite-se que dada uma configuração inicial, o problema consiste em se determinar uma correção para o problema localmente linear aproximando-se as parcelas não lineares através de séries de Taylor de ordem superior truncada (PRESS, et al., 1986; HEATH, 1997 ) .

\section{C.2 Método de Newton,}

Considere-se inicialmente um sistema unidimensional do tipo

$$
g(x)=0
$$

em que $\mathrm{g}(\mathrm{x})$ e x são escalares. Pretende-se determinar o valor de x para o qual a função $\mathrm{g}(\mathrm{x})$ se anula. Em termos geométricos, como mostra a Figura C.1, a solução da Equação $g(x)$ corresponde ao ponto em que a curva corta o eixo x. Aresolução desse problema pelo método de Newton segue os seguintes passos: 


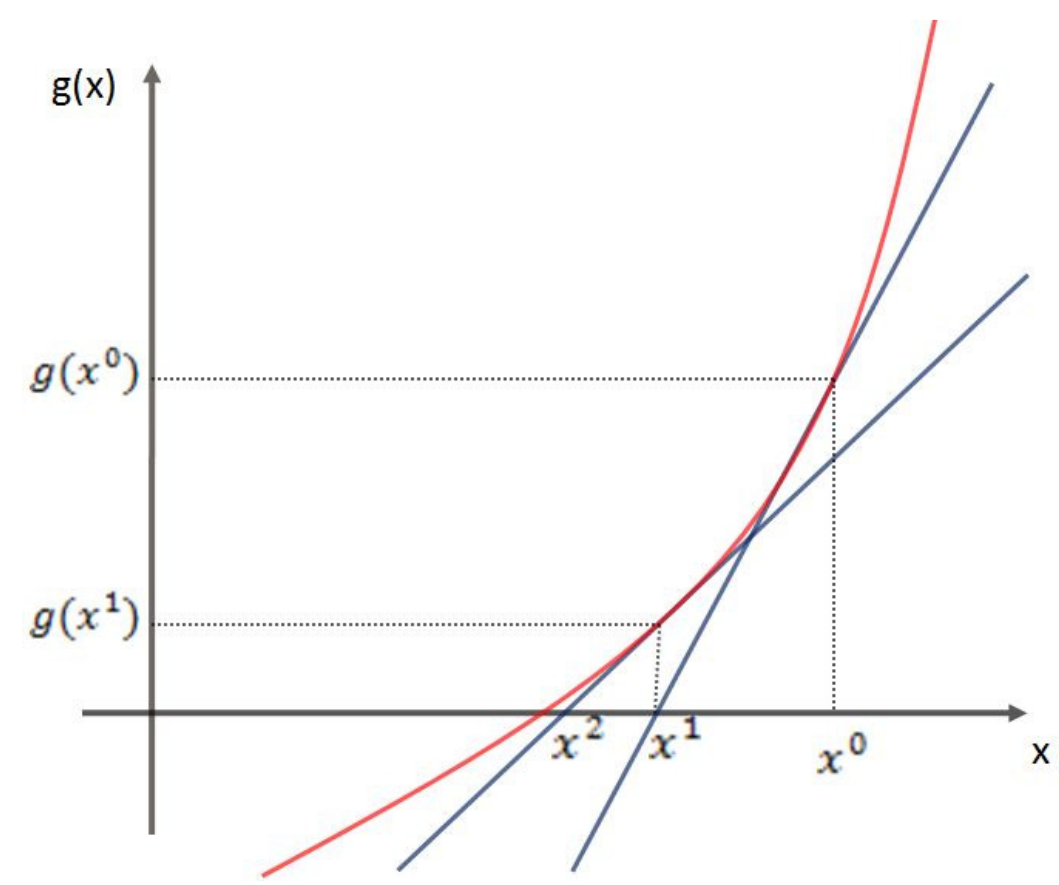

Figura C.1- Método de Newton

i. Fazer v=0 e escolher uma solução inicial $x=x^{(v)}=x^{(0)}$.

ii. Calcular o valor da função $\mathrm{g}(\mathrm{x})$ no ponto $x=x^{v}$.

iii. Comparar o valor da função $g\left(x^{v}\right)$ com a tolerância especificada $\varepsilon$ :

se $\left|g\left(x^{v}\right)\right| \leq \varepsilon$, então $x=x^{v}$ será a solução procurada dentro da faixa de tolerância $\pm \varepsilon ;$ se $\left|g\left(x^{v}\right)\right|>\varepsilon$, o algoritmo deverá prosseguir.

iv. Linearizar (ver fig. C.1) a função $\mathrm{g}(\mathrm{x})$ em torno do ponto $\left(x^{v} ; g\left(x^{v}\right)\right.$ ) por intermédio da série de Taylor:

$$
g\left(x^{v}+\Delta x^{v}\right) \cong g\left(x^{v}\right)+g^{\prime}\left(x^{v}\right) \Delta x^{v}
$$

sendo $g^{\prime}(x)=\partial g / \partial x$. Este passo se resume, de fato, ao cálculo da derivada $g^{\prime}\left(x^{v}\right)$.

v. Resolver o problema linearizado, ou seja, encontrar $\Delta x$ tal que:

$$
g\left(x^{v}\right)+g^{\prime}\left(x^{v}\right) \Delta x^{v}=0
$$

Isto significa que a nova estimativa de $\mathrm{x}$ passa a ser 


$$
x^{v+1}=x^{v}+\Delta x^{v}
$$

sendo

$$
\Delta x^{v}=-g\left(x^{v}\right) / g^{\prime}\left(x^{v}\right)
$$

vi. Fazer $v+1 \rightarrow v$ e voltar para o passo ii.

A variante do método de Newton ilustrada na Fig. C.2 é obtida considerando-se a derivada constante, isto é, no passo (iv) do algoritmo faz-se $g^{\prime}\left(x^{v}\right)=g^{\prime}\left(x^{0}\right)$. Nesta versão, o número de iterações, para uma dada tolerância de convergência, em geral é maior que no método original, mas cada uma das iterações se torna mais rápida pois a derivada não precisa ser recalculada a cada passo.

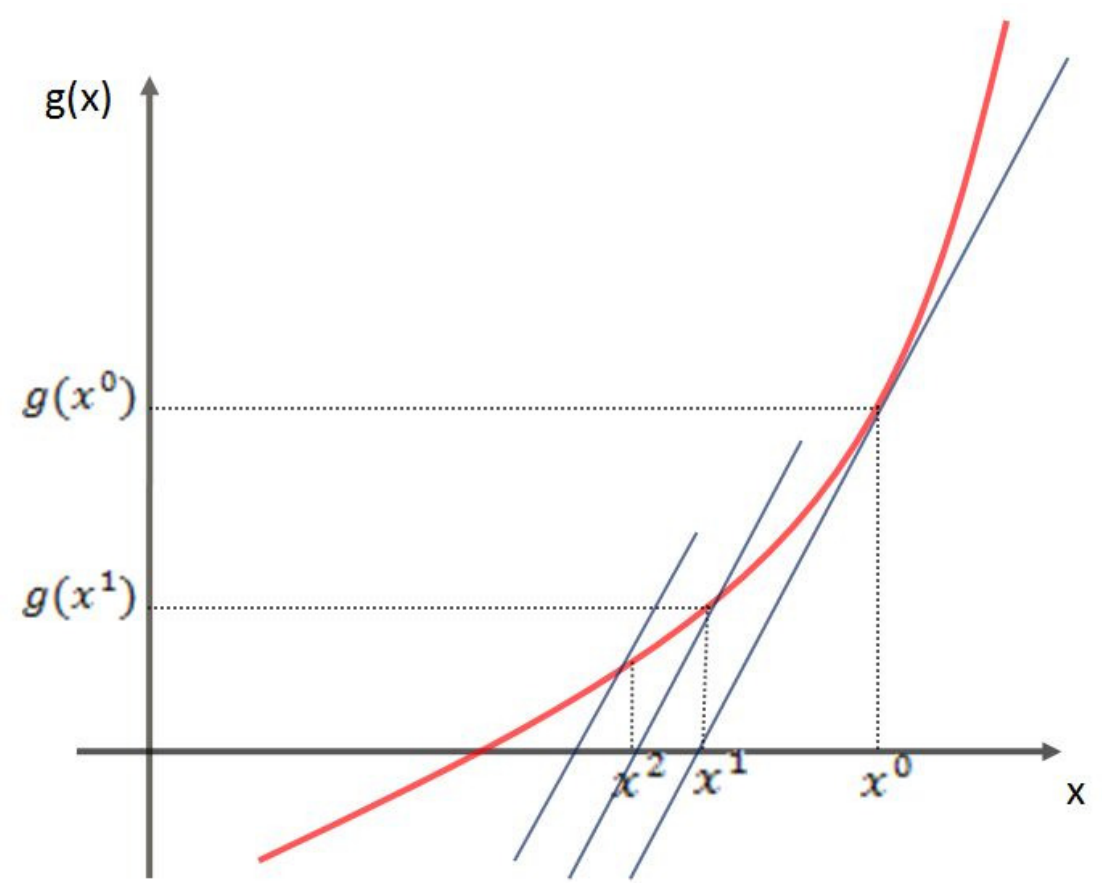

Figura C.2- Método de Newton com derivada constante

Considere-se agora a resolução do seguinte sistema n-dimensional:

$$
\underline{g}(\underline{x})=0
$$


sendo $\underline{g}(\underline{x})$ uma função vetorial (nx1) e $\underline{x}$ o vetor das incógnitas (nx1), ou seja,

$$
\begin{gathered}
\underline{g}(\underline{x})=\left[\left(g_{1}(\underline{x}), g_{2}(\underline{x}), \ldots, g_{n}(\underline{x})\right]^{t}\right. \\
\underline{x}=\left[x_{1}, x_{2, \ldots,} x_{n}\right]^{t}
\end{gathered}
$$

A resolução da equação (C.6) segue, basicamente, os mesmos passos do algoritmo apresentado anteriormente para o caso unidimensional. A principal diferença está no passo (iv), no qual, agora, aparece a matriz jacobiana. A linearização da função vetorial $\underline{g}(\underline{\mathrm{x}})$ para $\underline{\mathrm{x}}=\underline{\mathrm{x}^{\mathrm{v}}}$ é dada pelos dois primeiros termos da série de Taylor.

$$
\underline{g}\left(\underline{x}^{v}+\Delta \underline{x}^{v}\right) \cong \underline{g}\left(\underline{x}^{v}\right)+J\left(\underline{x}^{v}\right) \Delta \underline{x}^{v}
$$

sendo a matriz jacobiana $\mathrm{J}$ dada por

$$
J=\partial g / \partial \underline{x}=\left[\begin{array}{llll}
\frac{\partial g_{1}}{\partial x_{1}} & \frac{\partial g_{1}}{\partial x_{2}} & \cdots & \frac{\partial g_{1}}{\partial x_{n}} \\
\frac{\partial g_{2}}{\partial x_{1}} & \frac{\partial g_{2}}{\partial x_{2}} & \cdots & \frac{\partial g_{2}}{\partial x_{n}} \\
\cdots & \cdots & \cdots & \frac{\partial g_{n}}{\partial g_{n}} \\
\frac{\partial x_{n}}{\partial x_{2}} & \cdots & \frac{\partial x_{n}}{\partial x_{2}}
\end{array}\right]
$$

$\mathrm{O}$ vetor de correção $\Delta \underline{x}$ é calculado impondo-se que

$$
\underline{g}\left(\underline{x}^{v}\right)+J\left(\underline{x}^{v}\right) \Delta \underline{x}^{v}=0
$$

que é maneira linearizada de se resolver o problema $g\left(\underline{x}^{v}+\Delta \underline{x}\right)=0$. No caso particular em que , por exemplo, n=2, a Equação (C.9) assume a forma: 


$$
\begin{aligned}
& g_{1}\left(x_{1}, x_{2}\right) \cong g_{1}\left(x_{1}^{v}, x_{2}^{v}\right)+\left.\frac{\partial g_{1}}{\partial x_{1}}\right|_{v} \Delta x_{1}^{(v)}+\left.\frac{\partial g_{1}}{\partial x_{2}}\right|_{v} \Delta x_{2}^{(v)} \\
& g_{2}\left(x_{1}, x_{2}\right) \cong g_{2}\left(x_{1}^{v}, x_{2}^{v}\right)+\left.\frac{\partial g_{2}}{\partial x_{1}}\right|_{v} \Delta x_{1}^{(v)}+\left.\frac{\partial g_{2}}{\partial x_{2}}\right|_{v} \Delta x_{2}^{(v)}
\end{aligned}
$$

o algoritmo para resolução do sistema de equações $\underline{g}(\underline{x})=0$ pelo método de Newton é:

i. $\quad$ Fazer $v=0$ e escolher uma solução inicial $\underline{x}=\underline{x}^{(v)}=\underline{x}^{(0)}$.

ii. Calcular $\underline{g}\left(\underline{x}^{v}\right)$.

iii. Testar convergência: se $\left|g_{i}\left(\underline{x}^{v}\right)\right| \leq \varepsilon$ para $\mathrm{i}=1$, n, o processo convergiu para solução $\underline{x}^{v}$, caso contrário, passar para (iv).

iv. Calcular matriz jacobiana $J\left(\underline{x}^{v}\right)$.

v. Determinar nova solução $\underline{x}^{(v+1)}$ :

$$
\begin{gathered}
\underline{x}^{v+1}=\underline{x}^{v}+\Delta \underline{x}^{v} \\
\Delta \underline{x}^{v}=-\left[J\left(\underline{x}^{v}\right)\right]^{-1} \underline{g}\left(\underline{x}^{v}\right)
\end{gathered}
$$

\section{C.3 Caracteristicas do método de Newton}

a) Número de iterações independe da dimensão do problema;

b) A convergência é mais garantida do que em métodos como Gauss-Seidel;

c) Tempo de computação depende do cálculo da matriz Jacobiana e de sua inversa;

d) Apresenta convergência quadrática, desde que observados os seguintes aspectos:

- A solução inicial $x^{0}$ deve estar próxima o suficiente da solução do sistema, caso contrário, o método diverge, a não ser que sejam tomadas providências no sentido de contornar tal problema. 
- A inversa de $\mathrm{J}\left(\underline{\mathrm{x}}^{\mathrm{v}}\right)$ existe em todas as iterações necessárias até a convergência ser atingida;

e) É sensivel à escolha do ponto inicial;

f) Pode apresentar multiplas soluções;

Sofre com problemas de mau condicionamento

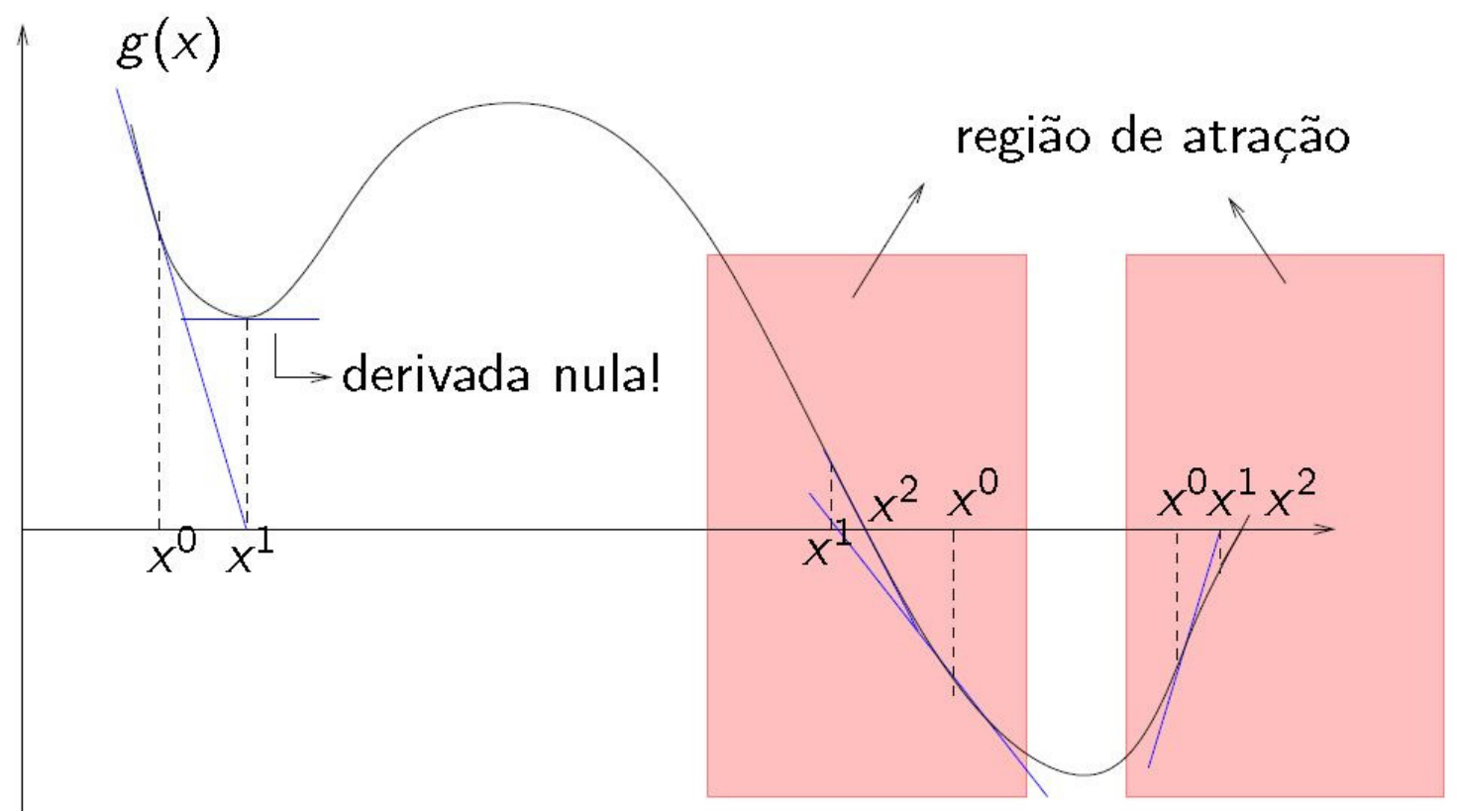

Figura C.3- convergencia do método de Newton 


\section{Apêndice D}

\section{O número de condição de uma matriz}

$\mathrm{Na}$ análise numérica, o número de condicionamento ou número de condição de um problema é uma medida indicando se o problema tem "boas condições" para ser tratado numericamente. Um problema com um número de condição pequeno é chamado de bem condicionado, enquanto os problemas que possuem um número de condição elevado são denominados mal condicionados.

\section{D.1 Introdução}

O número de condição associado ao sistema linear $\mathbf{A x}=\mathbf{b}$ é um número que estabelece uma estimativa da precisão que se pode obter para uma solução aproximada de $\mathrm{x}$. Note que isso é antes dos efeitos dos erros de arredondamento serem levados em consideração. O condicionamento é uma propriedade da matriz, não do algoritmo ou da precisão em ponto flutuante do computador usado para resolver o sistema correspondente.

Formalmente, o número de condicionamento de uma matriz inversível A é definido como produto da norma de A pela norma de sua inversa:

$$
\operatorname{cond}(\mathbf{A})=\|\mathbf{A}\|\left\|\mathbf{A}^{-\mathbf{1}}\right\|
$$

\section{D.2 Normas de Matrizes e Condicionamento}

Para além do problema de instabilidade numérica que decorre da propagação dos erros de arredondamento, podem também ocorrer problemas de mau condicionamento. Para podermos analisar convenientemente o problema do condicionamento, é necessário introduzirmos a noção de normas vectoriais e matriciais. 
Definição: Seja $\|$.$\| uma norma vectorial em \mathrm{Rn}$. A norma da matriz A induzida pela norma vectorial é:

$$
\|\mathbf{A}\|=\max _{\|\mathbf{x}\|=1}\|\mathbf{A} \mathbf{x}\|=\max _{\mathbf{x} \neq 0} \frac{\|\mathbf{A} \mathbf{x}\|}{\|\mathbf{x}\|}
$$

\section{Propriedades :}

Para quaisquer matrizes A, $\mathbf{B}$ quadradas e qualquer vector $\mathbf{x}$, temos:

i) $\|\mathbf{A} \mathbf{x}\| \leq\|\mathbf{A}\|\|\mathbf{x}\|$

ii) $\|\mathbf{A} \mathbf{B}\| \leq\|\mathbf{A}\|\|\mathbf{B}\|$

\section{demostração:}

i) É imediato. Caso $\mathbf{x} \neq \mathbf{0}$, temos $\|\mathbf{A}\| \geq\|\mathbf{A} \mathbf{x}\| /\|\mathbf{x}\|<=>\|\mathbf{A} \mathbf{x}\| \leq\|\mathbf{A}\|\|\mathbf{x}\|$, e se $\mathbf{x}=\mathbf{0}$ temos $0 \leq 0$.

ii) É semelhante, basta ver que de i), para qualquer $\mathbf{x} \neq \mathbf{0}$,

$\|\mathbf{A} \mathbf{B} \mathbf{x}\| /\|\mathbf{x}\| \leq\|\mathbf{A}\|\|\mathbf{B} \mathbf{x}\| /\|\mathbf{x}\|$

e portanto

$\|\mathbf{A} \mathbf{B}\|=\max \|\mathbf{A} \mathbf{B} \mathbf{x}\| /\|\mathbf{x}\| \leq\|\mathbf{A}\| \max \|\mathbf{B} \mathbf{x}\| /\|\mathbf{x}\|=\|\mathbf{A}\|\|\mathbf{B}\|$.

\section{Exemplos de Normas :}

1) Norma do Máximo (linhas): $\max$

Norma vectorial, $\|\mathbf{x}\|_{\infty}=\quad \mathrm{i}=1, \ldots, \mathrm{n}$

Norma matricial induzida, $\|\mathbf{A}\|_{\infty}=\max _{i=1, \ldots, n}^{N}\left|a_{i j}\right|$ 
Corresponde ao máximo dos somatórios dos módulos dos elementos das linhas.

2) Norma da Soma (colunas):

$\mathrm{N}$

Norma vectorial, $\|\mathbf{x}\|_{1}=\sum\left|x_{\mathrm{i}}\right|$

$$
\mathrm{i}=1
$$

Norma matricial induzida, $\|\mathbf{A}\|_{1}=\max _{j=1, \ldots, n}^{N}\left|a_{i j}\right|$

Corresponde ao máximo dos somatórios dos módulos dos elementos das colunas.

3) Norma Euclidiana:

$\mathrm{N}$

Norma vectorial, $\|\mathbf{x}\|_{2}=\left(\sum\left|\mathrm{x}_{\mathrm{i}}\right|^{2}\right)^{1 / 2}$

$$
\mathrm{i}=1
$$

Norma matricial induzida, $\|\mathbf{A}\|_{2}=\rho\left(\mathbf{A}^{\mathbf{T}} \mathbf{A}\right)^{1 / 2}$

onde $\rho(\mathbf{A})$ designa o raio espectral da matriz $\mathbf{A}$.

\section{Definição:}

Designamos por raio espectral de uma matriz $\mathbf{A}$ o valor :

$$
\rho(\mathbf{A})=\max _{\mathrm{i}=1, \ldots, \mathrm{n}}\left|\lambda_{\mathrm{i}}\right|
$$


onde $\lambda_{1}, \ldots, \lambda_{\mathrm{n}}$ são os valores próprios de $\mathbf{A}$.

Observação: Se a matriz $\mathbf{A}$ for simétrica então $\|\mathbf{A}\|_{2}=\rho(\mathbf{A})$.

Teorema: Seja A uma matriz quadrada.

- Para qualquer norma matricial II.I , temos

$$
\rho(\mathbf{A}) \leq\|\mathbf{A}\|
$$

- Para qualquer $\varepsilon>0$ existe sempre uma norma induzida II.II tal que:

$$
\|\mathbf{A}\| \leq \rho(\mathbf{A})+\varepsilon
$$

Ou seja, o raio espectral é o ínfimo do conjunto das normas induzidas de uma matriz.

\section{D.3 Número de Condição de uma Matriz}

Ao resolver um sistema $\mathbf{A} \mathbf{x}=\mathbf{b}$ podemos ter problemas de condicionamento e de estabilidade numérica. Os problemas de estabilidade numérica estão relacionados com o algoritmo que utilizamos para resolver o sistema. Por exemplo, para evitar os problemas de instabilidade numérica, é habitual considerar o método de eliminação de Gauss com pesquisa de pivot. No entanto, se o problema fôr mal condicionado, essas técnicas de pesquisa de pivot deixam de ser úteis, já que um problema mal condicionado será sempre numericamente instável. Interessa-nos, portanto, identificar quais os sistemas que nos podem trazer problemas de condicionamento.

Veremos que é possível identificar de uma forma relativamente simples os sistemas mal condicionados. Admitamos que os elementos de $\mathbf{A}$ e de $\mathbf{b}$ no sistema estão afectados de erros. Então o sistema que efectivamente resolvemos não é $\mathbf{A x}=\mathbf{b}$ mas sim um ligeiramente diferente ou perturbado!

Denotemos por $\delta_{\mathbf{A}}$ e $\delta_{\mathbf{b}}$, respectivamente, as perturbações da matriz $\mathbf{A}$ e do segundo membro b. Pretendemos saber o efeito desses erros (perturbações) no vector $\mathbf{x}$, solução de 


$$
\mathbf{A x}=\mathbf{b}
$$

Voltando ao efeito das perturbações no vector solução de $\mathbf{A x}=\mathbf{b}$, podemos escrever o sistema perturbado

$$
\left(\mathbf{A}+\delta_{\mathrm{A}}\right)\left(\mathbf{x}+\delta_{\mathbf{x}}\right)=\mathbf{b}+\delta_{\mathbf{b}}
$$

em que se designou por $\left(\mathbf{x}+\delta_{\mathbf{x}}\right)$ a solução do sistema perturbado.

\section{Perturbações no segundo membro}

Com o objectivo de simplificar a exposição, vamos considerar primeiro o caso particular em que $\delta_{\mathrm{A}}=0$ (isto é, as perturbações verificam-se apenas no segundo membro b).

Assim, seja o sistema perturbado

$$
\mathbf{A}\left(\mathbf{x}+\delta_{\mathbf{x}}\right)=\mathbf{b}+\delta_{\mathbf{b}} .
$$

Como $\mathbf{A x}=\mathbf{b}$ e se admite $\mathbf{A}$ invertível, teremos

$$
\delta_{\mathrm{x}}=\mathbf{A}^{-1} \delta_{\mathrm{b}}
$$

Tomando normas

$$
\left\|\delta_{\mathbf{x}}\right\| \leq\left\|\mathbf{A}^{-1}\right\|\left\|\delta_{\mathbf{b}}\right\|
$$

O valor de $\left\|\delta_{\mathbf{x}}\right\|$ só por si não significa muito! Um erro de $\left\|\delta_{\mathbf{x}}\right\|=1$ numa solução tal que $\|\mathbf{x}\| \approx 10^{6}$ é aceitável enquanto o mesmo erro numa solução tal que $\|\mathbf{x}\| \approx 1$ é um desastre computacional!

Faz portanto mais sentido trabalhar com erros relativos os quais, em face do carácter vectorial de $\mathbf{x}$, definiremos por $\frac{\left\|\delta_{\mathbf{x}}\right\|}{\|\mathbf{x}\|}$ e perturbações relativas definidas por $\frac{\left\|\delta_{\mathbf{b}}\right\|}{\|\mathbf{b}\|}$, considerando $\mathbf{x} \neq 0$ e $\mathbf{b} \neq 0$ 
Então

$$
\frac{\left\|\delta_{\mathbf{x}}\right\|}{\|\mathbf{x}\|} \leq\left\|\mathbf{A}^{-1}\right\| \frac{\left\|\delta_{\mathbf{b}}\right\|}{\|\mathbf{x}\|}
$$

Mas de $\mathbf{A x}=\mathbf{b}$, aplicando normas

$$
\|\mathbf{b}\| \leq\|\mathbf{A}\|\|\mathbf{x}\|
$$

ou seja,

$$
\|\mathbf{x}\| \geq \frac{\|\mathbf{b}\|}{\|\mathbf{A}\|}
$$

Atendendo a (D.4) podemos escrever

$$
\frac{\left\|\delta_{\mathbf{x}}\right\|}{\|\mathbf{x}\|} \leq\left\|\mathbf{A}^{-1}\right\|\|\| \mathbf{A} \| \frac{\left\|\delta_{\mathbf{b}}\right\|}{\|\mathbf{b}\|}
$$

A quantidade que relaciona os erros relativos com as perturbações relativas é $\|\mathbf{A}\||| \mathbf{A}^{-1}||$ e costuma ser designada por número de condição da matriz $\mathbf{A}$. Será denotado por

$$
\text { cond } \mathbf{A}=\|\mathbf{A}\|\left\|\mathbf{A}^{-1}\right\|
$$

O valor numérico de cond $\mathbf{A}$ depende obviamente da norma utilizada. Quanto maior for cond A mais sensível é o sistema de equações a perturbações do segundo membro.

Matrizes com um número de condição elevado dizem-se mal condicionadas. Matrizes com um número de condição baixo dizem-se bem condicionadas.

Consequentemente, para matrizes cujo número de condição seja elevado, um pequeno erro relativo no vector $\mathbf{b}$, pode provocar um grande erro relativo na solução do sistema.

Pode provar-se que cond $\mathbf{A} \geq 1$. 
Perturbação da matriz

Seja o sistema perturbado

$$
\left(\mathbf{A}+\delta_{\mathbf{A}}\right) \cdot\left(\mathbf{x}+\delta_{\mathbf{x}}\right)=\mathbf{b} .
$$

Assim

$$
\delta_{\mathbf{x}}=-\mathbf{A}^{-1} \delta_{\mathbf{A}}\left(\mathbf{x}+\delta_{\mathbf{x}}\right) .
$$

Aplicando normas, podemos escrever

$$
\left\|\delta_{\mathbf{x}}\right\| \leq\left\|\mathbf{A}^{-1}\right\|\left\|\delta_{\mathbf{A}}\right\|\left\|\mathbf{x}+\delta_{\mathbf{x}}\right\|,
$$

ou seja,

$$
\frac{\left\|\delta_{\mathbf{x}}\right\|}{\left\|\mathbf{x}+\delta_{\mathbf{x}}\right\|} \leq \underbrace{\|\mathbf{A}\|\|\| \mathbf{A}^{-1} \|}_{\text {cond } \mathbf{A}} \| \frac{\left\|\delta_{\mathbf{A}}\right\|}{\|\mathbf{A}\|}
$$

e, mais uma vez, constatamos que é o número de condição que determina a maior ou menor influência que a perturbação da matriz A pode ter na solução. 


\section{Apêndice E}

Neste apêndice apresenta-se a técnica de escalonamento de linhas e colunas (JENNINGS, 1985) que foi implementada neste trabalho.

\section{E.1 Escalonamento de linhas e colunas}

Se cada equação de um conjunto linear $A x=b$ for escalonada, este tem o efeito de escalonamento das linhas da matriz de coeficientes. Se $r_{i}$ for o fator de escalonamento para a equação $i$, então a equação torna-se-á:

$$
\left[\begin{array}{lll}
r_{1} a_{11} & r_{1} a_{12} \ldots \ldots \ldots \ldots . . & r_{1} a_{1 n} \\
r_{2} a_{21} & r_{2} a_{22} \ldots \ldots \ldots \ldots . . & r_{2} a_{2 n} \\
\cdot & \cdot & \cdot \\
r_{n} a_{n 1} & r_{n} a_{n 2} \ldots \ldots \ldots \ldots . & r_{n} a_{n n}
\end{array}\right]\left[\begin{array}{l}
x_{1} \\
x_{2} \\
\cdot \\
x_{3}
\end{array}\right]=\left[\begin{array}{l}
r_{1} b_{1} \\
r_{2} b_{2} \\
\cdot \\
r_{n} b_{n}
\end{array}\right]
$$

Por outro lado, se cada variável $x_{i}$ for substituída por $x_{i} / c_{i}$, o efeito nos coeficientes da matriz será o escalonamento nas colunas. Com ambas linhas e colunas escalonadas a equação torna-se:

$$
\left[\begin{array}{lll}
r_{1} a_{11} c 1 & r_{1} a_{12} c_{2} \ldots \ldots \ldots \ldots . . & r_{1} a_{1 n} c_{n} \\
r_{2} a_{21} c_{1} & r_{2} a_{22} c_{2} \ldots \ldots \ldots \ldots . . . & r_{2} a_{2 n} c_{n} \\
\cdot & \cdot & \cdot \\
r_{n} a_{n 1} c_{1} & r_{n} a_{n 2} c_{2} \ldots \ldots \ldots \ldots . & r_{n} a_{n n} c_{n}
\end{array}\right]\left[\begin{array}{l}
x_{1} / c_{1} \\
x_{2} / c_{2} \\
\cdot \\
x_{3} / c_{n}
\end{array}\right]=\left[\begin{array}{l}
r_{1} b_{1} \\
r_{2} b_{2} \\
\cdot \\
r_{n} b_{n}
\end{array}\right]
$$

Se $\mathrm{R}$ e $\mathrm{C}$ forem matrizes diagonais de fatores de escalonamento de linhas e colunas respectivamente, a equação modificada (E.2) poderá ser escrita como :

$$
\bar{A} \bar{x}=\bar{b}
$$


onde $\bar{A}=R A C, \bar{b}=R b$ e $x=C \bar{x}$.

A matriz simétrica pode ser escalonada, simetricamente, fazendo $r_{i}=c_{i}$. Se uma matriz simétrica definida positiva for escalonada de tal modo que $r_{i}=c_{i}=a_{i i}^{-1 / 2}$, então a matriz resultante terá uma diagonal contendo apenas elementos unitários. Alem disso, usando a propriedade de uma matriz definida positiva que $a_{i j}^{2}<a_{i i} a_{j j}$, define-se:

$$
\left|\bar{a}_{i j}\right|=\left|r_{i} a_{i j} c_{j}\right|=a_{i i}^{-1 / 2} a_{j j}^{-1 / 2}\left|a_{i j}\right|<1
$$

Assim, o escalonamento simétrico, de uma matriz simétrica definida positiva, que torna unitário todos os elementos da diagonal principal, assegura que todos os elementos fora da diagonal tenham um módulo menor que a unidade.

O escalonamento pode ter um efeito marcado na escolha de pivôs onde a seleção de pivô é adotada. Por exemplo, se a matriz.

$$
A=\left[\begin{array}{ccc}
-0.001 & 1 & 1 \\
1 & 0.78125 & 0 \\
1 & 0 & 0
\end{array}\right]
$$

é escalonada usando as matrizes $\mathrm{R}=\mathrm{C}=\left[\begin{array}{ll}2000 & 1 \\ 1 & 1\end{array}\right]$, então

$$
\bar{A}=\left[\begin{array}{ccc}
-4000 & 2000 & 2000 \\
2000 & 0.78125 & 0 \\
2000 & 0 & 0
\end{array}\right]
$$

Conseqüentemente, o elemento não nulo menor de A será convertido em um elemento maior de $\bar{A}$. Na realidade, é possível converter qualquer elemento não nulo de uma matriz no elemento de maior magnitude da matriz escalonada, adotando fatores de escalonamento de linhas e colunas satisfatórios. 